\title{
Teacher-student Relationships in Diverse New Zealand Year 10 Mathematics Classrooms: Teacher Care
}

\author{
By \\ Robin Margaret Averill
}

\begin{abstract}
A thesis
submitted to the Victoria University of Wellington

in fulfilment of the requirements for the degree of

Doctor of Philosophy in

Mathematics Education
\end{abstract}

Victoria University of Wellington

September, 2009 


\section{Abstract}

Teacher-student relationships are considered influential for academic achievement and motivation, particularly for students of minority and low socio-economic groups. Teacher care is an essential component of effective teacher-student relationships. This study examined factors that contribute to developing and maintaining caring teacher-student relationships in low socio-economic multicultural classrooms (Māori, Pasifika, New Zealand European). Three areas of teacher care were explored: care for students as individuals, their mathematical progress, and for students as culturally located individuals.

The sample comprised three urban schools, one class and one teacher in each school for each of two years (six Year 10 mathematics teachers and their classes in total). Three data collection periods were used: the initial four weeks of the school year, and two weeks late in each of school terms 2 and 3. Each data collection period included classroom observations, teacher and student interviews, and teacher and student questionnaires.

Within a holistic context of classroom well being, characteristics of caring teacherstudent relationships were found to fit within four dispositional aspects (liking, respecting, and being tolerant of each other, and being able to reflect one's personal identity), and four themes (knowing each other as people, knowing each other as learners, knowing each other's cultures, and enhancing feelings of cultural identity). Specific classroom practices found to be supportive of respectful caring teacher-student relationships included using humour, one-to-one teacher-student interactions, making opportunities for sharing personal identities, and expecting mathematical progress.

Mixed results were obtained regarding how deeply students value their heritage cultures, whether or not they believe these are well reflected in their schools and classrooms, and the extent to which they would like them to be reflected in these places.

There is evidence that for many Māori, Pasifika, and low socio-economic students, mathematics teachers can enhance students' motivation and mathematical achievement by using explicitly caring practices. Teachers must acknowledge and attend to caring teaching approaches to maximise their students' progress in, and enjoyment of, mathematics. 


\section{Acknowledgements}

My sincere thanks first and foremost go to Megan Clark for her careful and insightful supervision of this study. Megan, I am very grateful for your wisdom, patience, constant good humour and encouragement, and your friendship. My heartfelt thanks, Megan.

I am also indebted to Herewini Easton and Fuapepe Rimoni for very graciously allowing me to draw from their considerable wealth of cultural, educational, and research knowledge to help ensure the study was culturally responsive. Your willingness, good humour, helpful suggestions, time, and encouragement have been essential for this work and are very much appreciated. I have incorporated your advice to the best of my ability and know that any misinterpretations and mistakes in culturally linked ideas are mine alone. Thank you very much for your faith and trust in this study and your very valuable contributions.

Thanks also to my family, Christopher for your help in many ways: data entry, reading, and thinking about the work from a student's perspective, Matt for your clever ways with words and argument, and Mum for your constant support and interest.

I am very grateful to the New Zealand Institute of Mathematics \& its Applications, the Faculty of Education of Victoria University of Wellington, and the Victoria University Foundation for financial and practical support towards completing this research.

Thank you also to my colleagues, particularly Roger Harvey, for suggestions of useful literature, feedback on ideas, data gathering tools, and writing about this research, and Susan Kaiser, for your wonderful support and eagle eye. Many thanks.

Finally, this research would not have been possible without the huge contributions made by the six study teachers and their classes. The teachers allowed me to visit their classrooms on many occasions, interview them many times, and disturb their teaching in order to collect student data. The students willingly contributed to this study with their time, and their honest, open, and very valuable interview and questionnaire responses. I always felt welcome in your schools and classrooms, many thanks to you all. 


\section{Table of Contents}

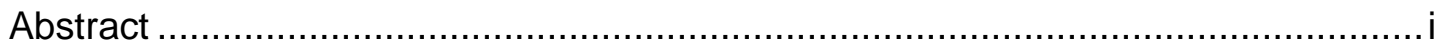

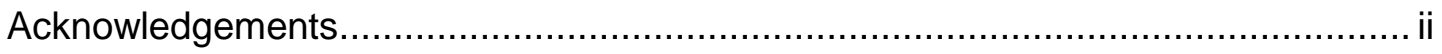

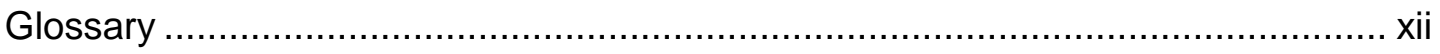

CHAPTER ONE Introduction: The Context of Teacher-Student Relationships.....1

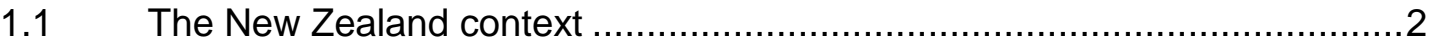

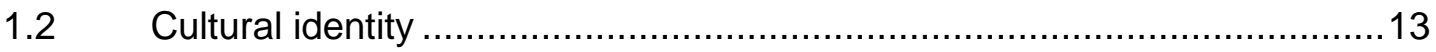

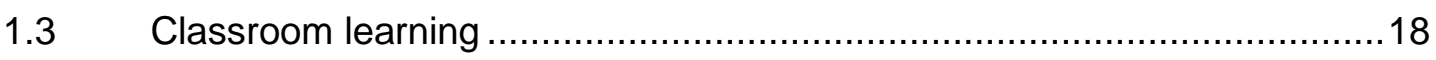

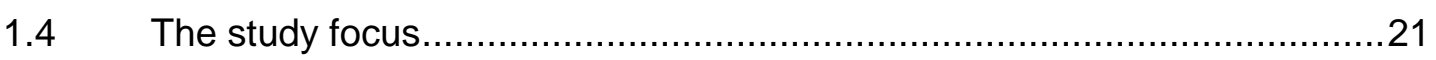

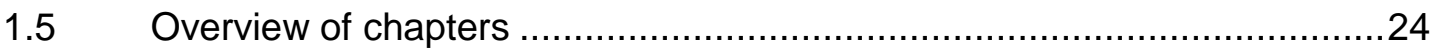

CHAPTER TWO Teacher Care: The Literature .................................................26

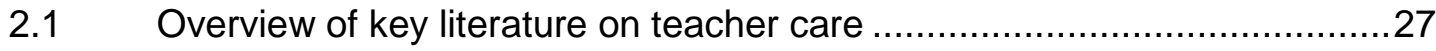

$2.2 \quad$ The importance of teacher care ...........................................................28

2.2.1 Modelling and teaching an important human value .....................28

2.2.2 Impact of teacher care on learning and achievement..................30

2.2.3 Impact of teacher care on affect and engagement.....................31

2.3 Students' sense of being cared for at school ......................................... 32

2.3.1 Caring school environments ....................................................33

2.3.2 Caring classroom environments .............................................34

2.3.3 Behaviours of caring teachers ..............................................36

Care for students as individuals ....................................... 39

Care for students' mathematics learning ...............................41

Caring for students as culturally located individuals ................42

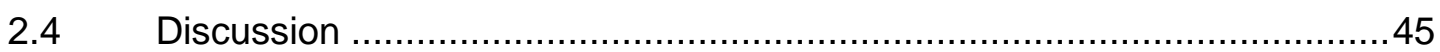

CHAPTER THREE Methodology and Implementation .....................................46

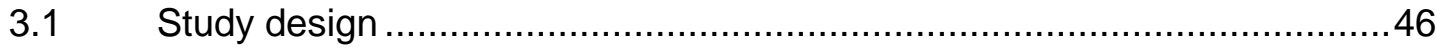

3.1.1 Data collection methods ...................................................... 50

3.1.2 Researcher-participant relationships ..................................57

3.2 Developing culturally responsive data gathering tools ............................58

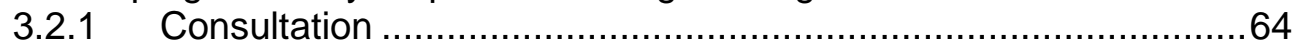

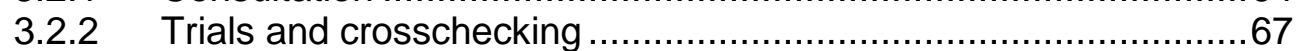

3.2.3 Discussion .....................................................................

3.3 Ethical considerations and study participants ...................................... 71

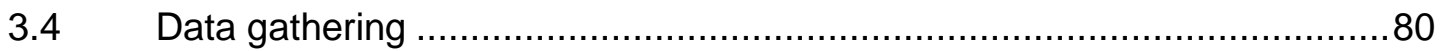

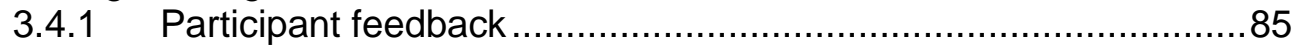

3.5 Data analysis and description ............................................................. 86

3.5.1 The Teacher Care Mathematics Whare (house): A model integrating the three study typologies 
CHAPTER FOUR Study Schools, Teachers, and Classes.................................95

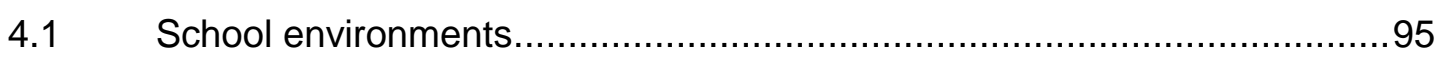

4.2 Teachers, classes, and classroom environments ................................103

4.3 Study teachers' teaching of mathematics .......................................... 108

4.4 Students' ethnicities: Student data vs school data................................112

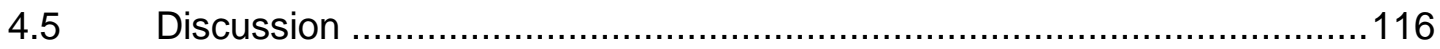

CHAPTER FIVE The Mathematics Lessons …................................................116

5.1 Comparison of the two most and two least caring classrooms ...................118

5.1.1 Taha Hinengaro.................................................................121

5.1.2 Taha Whānau...................................................................123

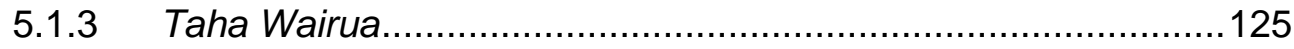

5.1.4 Taha Tinana ..................................................................... 127

5.1.5 A holistic view of the tapa wha model of teacher care.................129

5.2 Care for mathematics learning ...................................................... 131

5.2.1 Supporting new mathematical learning .................................134

5.2.2 Reinforcing mathematical learning..........................................137

5.2.3 Ensuring relevance of mathematical learning ..........................141

5.2.4 Giving encouragement, assistance, and feedback..................147

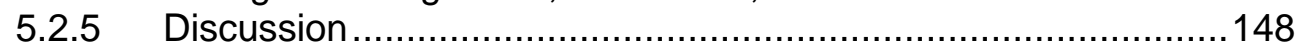

5.3 Caring relationships in action: An example of a lesson exhibiting

$5.4 \quad$ Care for students as culturally located individuals ............................... 156

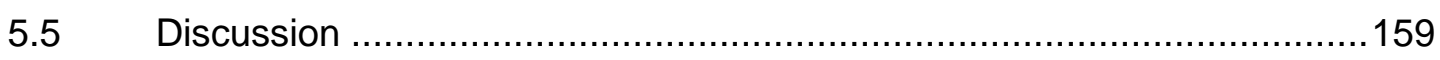

CHAPTER SIX Teachers' Perceptions of their Practice ....................................160

6.1 Teacher-student relationships - teachers' views .................................. 162

6.2 Establishing and maintaining rapport .............................................. 163

6.3 Cultural identity and learning mathematics - teachers' views ..................172

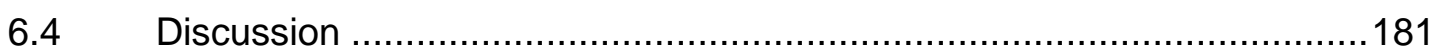

CHAPTER SEVEN Students' Perceptions of Caring Mathematics Teachers..... 182

$7.1 \quad$ Dispositional themes ............................................................................ 183

7.1.1 Liking each other: "He gets along with everyone, that's one of the good things"................................................................... 183

7.1.2 Respecting each other: "We all respect him because he respects us"....

7.1.3 Being tolerant of each other: "Maybe there's something that we won't understand and she needs to know that".........................191

7.1.4 Being able to reflect personal identity: "Cause it was just funny, and everyone was laughing, it was yeah...happy"..... 194 
7.2 Culturally responsive themes .......................................................196

7.2.1 Knowing each other: "I'd just tell them straight up and then they'll understand" ................................................................196

7.2.2 Knowing each other as learners: "students have different

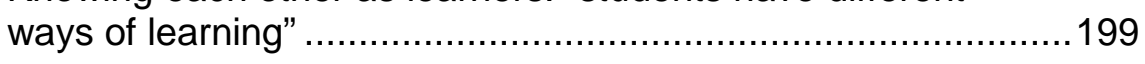

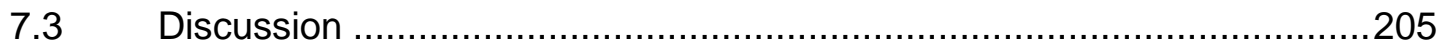

CHAPTER EIGHT Students as Culturally Located Individuals .......................207

8.1 Students' cultural identities..............................................................207

8.1.1 Students' depth of engagement with their heritage culture/s......208

8.1.2 Students' perceptions of the relevance of their heritage culture to their mathematics learning................................................212

8.2 Students' perceptions of the importance placed on heritage cultures at

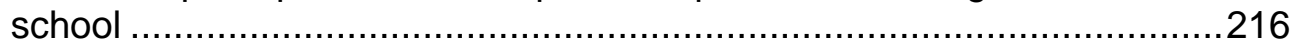

8.2.1 Students' perceptions of the importance placed on students' culture by their school and by their teacher .............................216

8.2.2 Students' perceptions of their teachers' knowledge of their cultures and the Māori and Pacific worlds. 220

8.3 Students' perceptions of links between students' cultures and mathematics

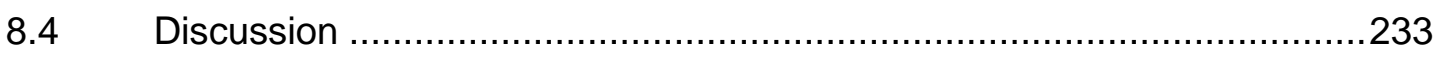

CHAPTER NINE Classroom Practices that Foster a Caring Environment .....235

9.1 Practices that support caring classrooms ..........................................235

9.1.1 One-to-one teacher-student interactions.................................235

9.1.2 Making opportunities for sharing personal identities ...................237

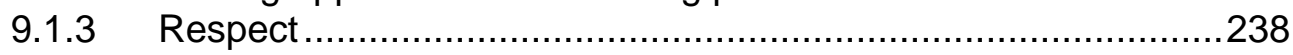

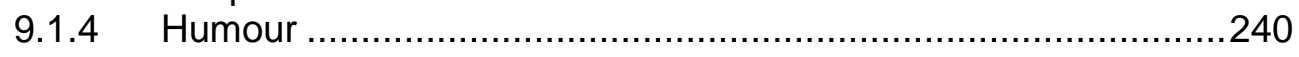

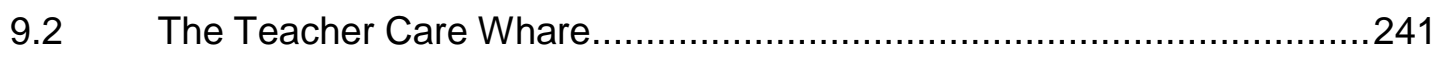

CHAPTER TEN Discussion and Conclusion ................................................245

10.1 Issues affecting the research process and results ..............................245

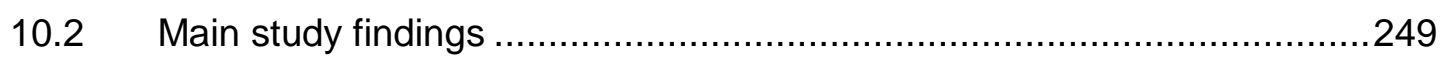

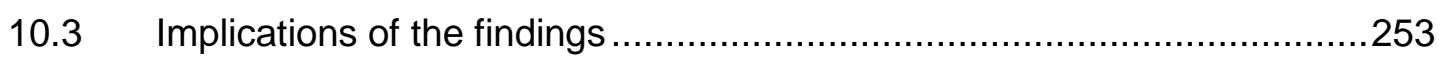

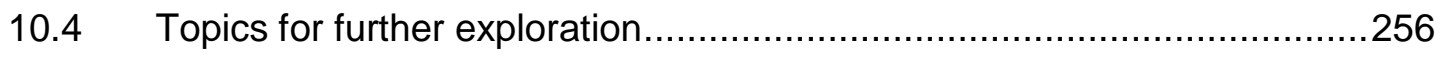

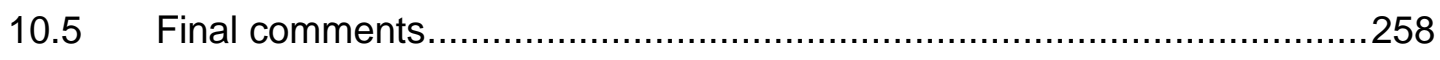

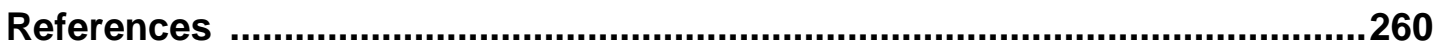




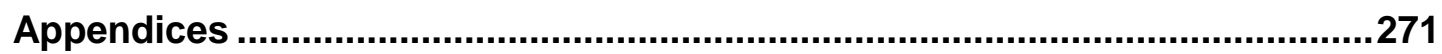

Appendix 1: Literature (main empirical studies) ............................................271

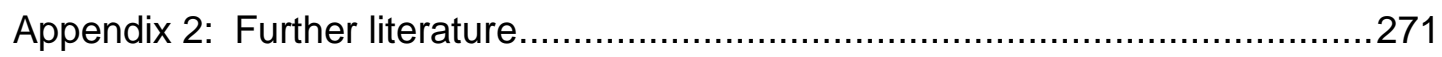

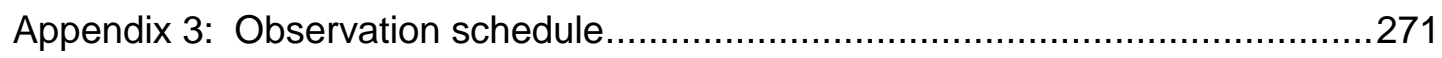

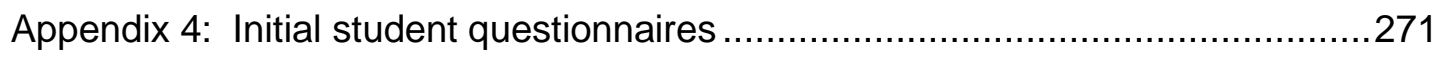

Appendix 5: Second student questionnaires ...............................................271

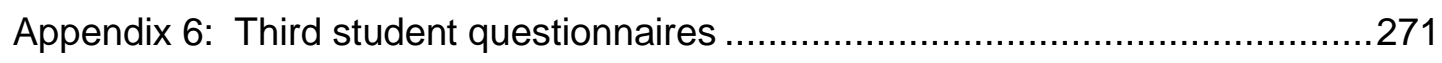

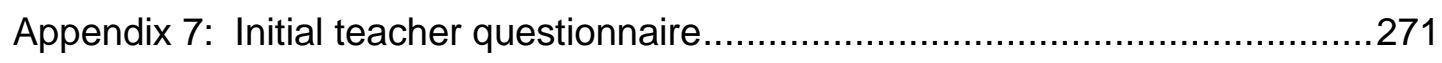

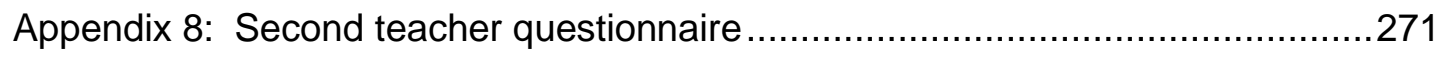

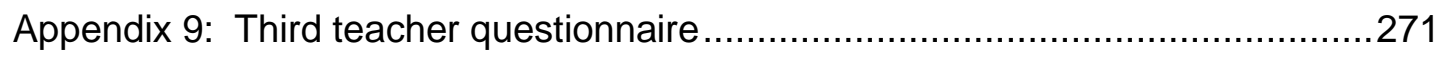

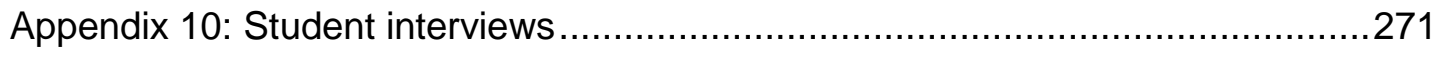

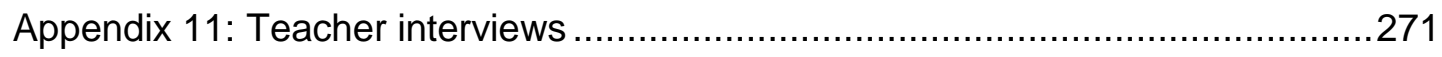

Appendix 12: Cross-checking themes .......................................................271

Appendix 13: Participant information and consent form (sample)........................271

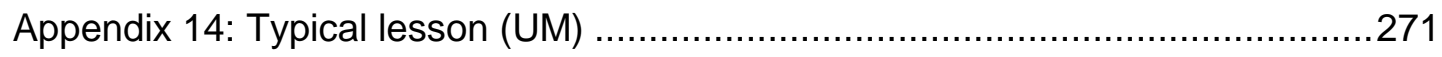

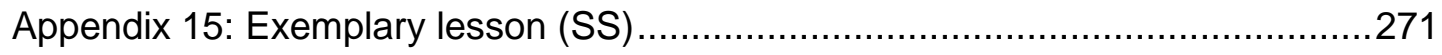




\section{List of Tables}

Table 3.1: Reasons for rejection of potential paradigms …..........................47

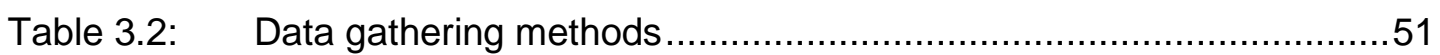

Table 3.3: Modifications to the observation schedule as a result of

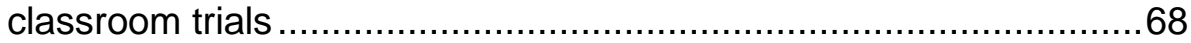

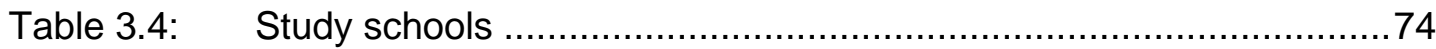

Table 3.5: $\quad$ Summary of study participants ................................................... 76

Table 3.6: $\quad$ Main data types, analysis methods, theoretical foundations, and thesis locations of analysed data .............................................. 89

Table 4.1: $\quad$ Schools' interpersonal environments...............................................96

Table 4.2: $\quad$ Schools' Māori dimensions: Comments from Education Review Office reports

Table 4.3: $\quad$ Schools' Pasifika dimensions: Comments from Education Review

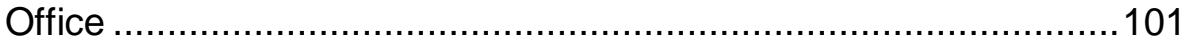

Table 4.4: Study teachers' lessons (mathematics teaching and learning) .......108

Table 4.5: Study teachers' lessons (teachers' expectations and use of praise) ...110

Table 5.1: Viewing the observation data using Durie's (1998) whare tapa wha model for health and well-being .......................................... 119

Table 5.2: $\quad$ Framework used to analyse lesson audio recordings .....................132

Table 6.1: Teacher perceptions of student characteristics contributing to teacher-student rapport...................................................... 168

Table 6.2: Importance to teachers of the groups they belong to......................175

Table 6.3: Knowing about and using Māori and Pasifika examples in

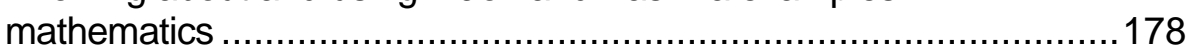

Table 8.1: Importance to students of the groups they belong to .....................212

Table 8.2: How study schools show that cultures are important.....................217

Table 8.3: How study teachers show that cultures are important ...................218 


\section{List of Figures}

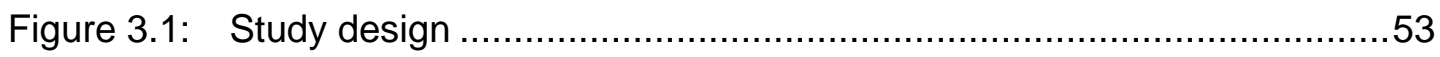

Figure 3.2: The data gathering tool development process ................................63

Figure 3.3: The Teacher Care Mathematics Whare ...........................................91

Figure 3.4: Typology for lesson elements (the whiteboard of the whare) ..............92

Figure 3.5: Typology of interpersonal elements (the whāriki of the whare) ...........93

Figure 4.1: Students' ethnicity as recorded in Ministry of Education data ............114

Figure 4.2: Students' self-reported ethnicity .................................................. 114

Figure 5.1: The Teacher Care Mathematics Whare .............................................118

Figure 5.2: Transcript from an excerpt of a lesson given by UM .......................135

Figure 5.3: Transcript from an excerpt of a lesson given by SS ....................... 140

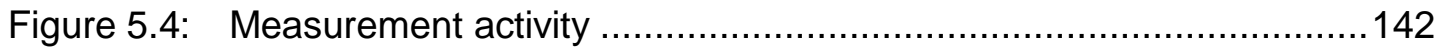

Figure 5.5: Excerpt from a lesson on solving equations ................................147

Figure 5.6: Percentages of lesson time intervals incorporating lesson elements for WK (excellent lesson) and UU (typical lesson) .............149

Figure 5.7: Blackboard message for students arriving into class .......................153

Figure 6.1: Matrix of coding themes .............................................................. 161

Figure 6.2: Importance to teachers of the groups they belong to ........................175

Figure 7.1: Students' responses to 'My teacher cares about me'........................185

Figure 7.2: Students' responses to 'My maths teacher wants to help me learn'..186

Figure 7.3: Students' responses to 'My teacher encourages me to do my best' 186

Figure 7.4: Students' responses to 'I like the way my teacher treats me when I make a mistake' .......................................................................190

Figure 7.5: Students' responses to 'My teacher treats me with respect' .............190

Figure 7.6: Responses to 'My teacher gives me feedback about my maths progress' (Questionnaire 3)

Figure 7.7: Responses to 'My teacher knows how good I am at maths'201 (Questionnaire 1)

Figure 7.8: Responses to 'My teacher knows how good I am at maths'

Figure 7.9: Responses to 'I like getting written feedback about my maths progress'.

Figure 7.10: Responses to 'I like my teacher telling me about my maths progress'

Figure 7.11: Responses to 'I am happy to ask my teacher about my maths progress'

Figure 7.12: Responses to 'I like my class to hear the teacher's comments about my work'.

Figure 8.1: My culture is important to me (Māori ${ }^{*}$ students) ................................210

Figure 8.2: My culture is important to me (Pasifika* students) .........................211

Figure 8.3: My culture is important to me (New Zealand European* students) ...211

Figure 8.4: Importance to students of the groups they belong to .......................213 
Figure 8.5: Responses to 'My culture is not relevant at school' (\%).................214

Figure 8.6: Responses to 'My culture is not relevant to my maths learning' (\%) ...214

Figure 8.7: Responses to 'It is important for my maths learning that my

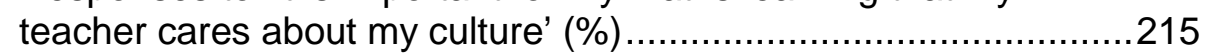

Figure 8.8: Responses to 'I would like my teacher to know my culture' (\%).....220

Figure 8.9: Responses to 'My teacher knows my culture'................................221

Figure 8.10: Responses to 'I would like my teacher to know about the Māori

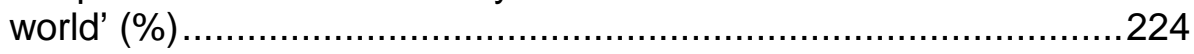

Figure 8.11: Responses to 'I would like my teacher to know about the Pacific world' (\%) .........................................................................225

Figure 8.12: Responses to 'My teacher knows about the Māori world' ................226

Figure 8.13: Responses to 'My teacher knows about the Pacific world' .............226

Figure 8.14: Responses to 'I would like my teacher to use Māori and Pacific examples in maths' (\%) ......................................................22

Figure 8.15: Responses to 'My teacher uses Māori and Pacific examples in maths' ....................................................................................228

Figure 9.1: The Teacher Care Mathematics Whare .....................................242

* See Glossary, p. x 


\section{Glossary}

New Zealand terms used in this thesis include:

\section{School decile}

The New Zealand Ministry of Education uses a numerical descriptor from 1 to 10 called 'school decile rating' (or 'decile') to indicate the socio-economic status of schools. Roughly ten percent of schools are given each decile rating, with a rating of 1 for the schools with the highest proportions of students from low socio-economic communities and 10 for the schools with the lowest proportion of these students (Ministry of Education, 2008a). (Section 1.1.5)

Culture

A culture is a shared way of life for a social group and includes shared knowledge, beliefs, values, perspectives, and behavioural norms (Banks, 2004; Nieto \& Bode, 2008). Discussing and thinking about culture is complex because every culture is inherently diverse, dynamic, and all of us are multicultural in that we move between different social groups. Throughout this thesis 'culture' refers to heritage cultures. (Section 1.2)

\section{Multicultural}

The term 'multicultural' is commonly used in New Zealand, and hence in this thesis, to refer to groups of people from a range of heritage cultures.

Integrated schools

New Zealand 'integrated' schools are those that have been integrated with the state education system. Many have a special (often religious) character. Students with first priority are those who meet the special character requirements of the school (Ministry of Education, 2008b).

\section{Pasifika}

The New Zealand Ministry of Education refers to people living in New Zealand who have strong cultural, heritage, and family connections to their Pacific Island countries of origin as 'Pasifika' peoples. Pasifika does not include Māori. 
The thesis contains some words in Māori and Pasifika languages (shown in italics). Translations for Māori and Pasifika words are given the first time the term appears, in parenthesis immediately following the term or, for longer translations, in footnotes. Translations of the terms most commonly used are provided below.

\begin{tabular}{|c|c|}
\hline Māori & \\
\hline ako & learning and teaching \\
\hline hinengaro & mind, intellect, heart \\
\hline iwi & Māori tribal affiliations \\
\hline manaakitanga & $\begin{array}{l}\text { nurturing relationships, looking after people, and being } \\
\text { careful how others are treated }\end{array}$ \\
\hline Pākehā & non-Māori New Zealanders \\
\hline taonga & treasures \\
\hline te Ao Māori & Māori worldview and values \\
\hline te reo Māori & the Māori language \\
\hline tikanga Māori & Māori practices \\
\hline tinana & body \\
\hline tuakana-teina & learning from more experienced peers \\
\hline wairua & spirit \\
\hline whānau & family \\
\hline whanaungatanga & relationships within a wider community \\
\hline whare & house \\
\hline whare tapa wha & literally, the four sided house \\
\hline whāriki & mat \\
\hline \multicolumn{2}{|l|}{ Tongan } \\
\hline mālie & an uplifting, energising atmosphere \\
\hline
\end{tabular}

In this thesis, Māori* $\left(\mathrm{M}^{\star}\right)$, Pasifika* $\left(\mathrm{P}^{\star}\right)$, New Zealand European* $\left(\mathrm{E}^{\star}\right)$ are used to indicate the ethnic heritages claimed by the student study participants, and Māori $(M)$, Pasifika $(P)$, New Zealand European (E) to indicate the student study participants' ethnicities as recorded by their school for reporting to the Ministry of Education (Section 4.4). An ' $N$ ' is used to indicate the student's ethnicity is unknown. 


\title{
CHAPTER ONE
}

\section{Introduction: The Context of Teacher-Student Relationships}

\author{
If they don't care, I won't. (Study student)
}

Learning is intellectual, social, and emotional (Stoll, Fink, \& Earl, 2003). For successful learning, all three aspects need deliberate teacher attention. Recent research (Bishop, Berryman, Tiakiwai, \& Richardson, 2003) affirms the crucial importance for many New Zealand students' learning of increasing secondary school teachers' focus on the social and emotional environment of their classrooms, particularly in terms of developing and nurturing caring, learning-focused teacherstudent relationships. Bishop et al.'s (2003) study focused on exploring factors key to the academic achievement of New Zealand Māori students. The study included data gathered from students in their first two years of secondary school and their whānau (family), teachers, and school principals. The study found positive teacherstudent relationships and quality teacher-student interactions to be the foundations for effective learning of these students.

Bishop et al.'s (2003) study is not alone in highlighting the importance of teacherstudent relationships. Many, both nationally (e.g., Gorinski, Ferguson, Wendt-Samu, \& Mara, 2008; Hawk \& Hill, 2000; Ocean, 2005) and internationally (e.g., Bosworth, 1995; Eccles, 2004; Ladson-Billings, 1990), also emphasise the place of effective teacher-student relationships for learning, for students in general and more crucially, for those often served less well in mathematics, indigenous and marginalised students:

\footnotetext{
Teacher-student relationships are a key component of classroom climate: high quality teacher-student relationships help facilitate academic motivation, school engagement, academic success, self-esteem, and more general socioemotional well-being (Eccles, 2004, p. 129).
}

There were two initial motivators for this study. Firstly effective teacher-student relationships are strongly linked to equitable classrooms in which teachers are well attuned to students' backgrounds and needs and are committed to having every student achieve (Anthony \& Walshaw, 2007; Holloway, 2004) (Chapter Two). A further powerful motivator is that there is persistent mention of teacher-student relationships in the literature regarding excellence in teaching (e.g., Education Review Office, 2002; Hattie, 2003; McCabe, 1995; Powell, 2000). Understanding more about effective teacher-student relationships will help inform educators how 
academic, emotional, and social aspects of teacher practice can be improved, and thereby enable the quality of, and equity in, student learning opportunities to be enhanced. The purpose of this study is therefore to explore the factors that contribute to establishing and nurturing effective teacher-student relationships within the context of New Zealand Year 10 multicultural mathematics classrooms.

Within the New Zealand education system the term 'multicultural' is used to describe schooling contexts (classes, schools, communities) with students from many cultural heritage groups. In order to explore teacher-student relationships between students of Māori, Pasifika, and New Zealand European heritage and their teachers, each of the schools selected for this study comprised roughly equal proportions of students from these three cultural groups (Chapter Three).

This chapter further outlines the rationale for, and the educational setting of, the study. It describes the New Zealand secondary school context (Section 1.1), cultural identity (Section 1.2), classroom learning (Section 1.3), the study focus and research questions (Section 1.4), and introduces the chapters to follow (Section 1.5).

\subsection{The New Zealand context}

In most New Zealand secondary schools, learning mathematics is compulsory until the end of Year 11, the first of three years of formal external qualifications. To gain the National Certificate of Educational Achievement (NCEA) ${ }^{1}$ at level 1 (Year 11), students must achieve eighty credits over all their subjects, including a minimum of eight mathematics credits (New Zealand Qualifications Authority, 2008). The majority of New Zealand students continue some form of mathematics study into Year 12.

This section briefly describes the place of mathematics teaching and learning within the New Zealand curriculum and the students who make up New Zealand's schools. Particular focus is given to the cultural contexts of students of Māori, Pasifika, and New Zealand European heritage, the target groups of this study.

Mathematics instruction in New Zealand is guided by a national curriculum statement (Ministry of Education, 1993a, 1993b, 2007a). The 1993 curriculum was in place at the time of data collection; however, the data will be interpreted in light of the current curriculum document in order to enhance relevance to future practice.

\footnotetext{
${ }^{1}$ New Zealand's qualification for secondary school students
} 
Although presented somewhat differently (e.g., three strands rather than six), there was little change to curriculum content or intended delivery style in the 2007 curriculum for mathematics and statistics education at Year 10.

The current curriculum includes the specific detail of mathematics and statistics to be taught alongside the content for other curriculum areas. In order to achieve the curriculum's vision of helping young people become "confident, connected, actively involved, lifelong learners" (Ministry of Education, 2007a, p. 7), specific "principles", "values", and "key competencies" (pp. 7, 9, 10, 12-13) (Table 1.1) are intended to guide and underpin the curriculum decision making in all curriculum areas. Teachers are required to plan, evaluate, and reflect on how they will address the curriculum components (Table 1.1).

Table 1.1: The curriculum context for mathematics and statistics education in New Zealand schools (Ministry of Education, 2007a)

\begin{tabular}{|l|l|}
\hline \multicolumn{1}{|c|}{ Curriculum component } & \multicolumn{1}{c|}{ Elements of curriculum component } \\
\hline $\begin{array}{l}\text { Principles ("foundations of } \\
\text { curriculum decision making") } \\
\text { (p. 9) }\end{array}$ & $\begin{array}{l}\text { High expectations, Treaty of Waitangi, Cultural diversity, } \\
\text { Inclusion, Learning to learn, Community engagement, } \\
\text { Coherence, Future focus }\end{array}$ \\
\hline $\begin{array}{l}\text { Values ("to be encouraged, } \\
\text { modelled and explored") } \\
\text { (p. 10) }\end{array}$ & $\begin{array}{l}\text { Excellence, Innovation, inquiry and curiosity, Diversity, } \\
\text { Equity, Community participation, Ecological sustainability, } \\
\text { Integrity, Respect }\end{array}$ \\
\hline $\begin{array}{l}\text { Key Competencies } \\
\text { ("capabilities for living and } \\
\text { lifelong learning") (p. 12) }\end{array}$ & $\begin{array}{l}\text { Thinking, Using language, symbols, and texts, Managing } \\
\text { self, Relating to others, Participating and contributing }\end{array}$ \\
\hline
\end{tabular}

Emphasis on developing inter-personal skills is given within all of the curriculum components. Set within the overall context of the curriculum, mathematics and statistics learning is now to have greater emphasis than that given by previous curricula (e.g., Ministry of Education, 1993a) on the social and emotional aspects of learning.

Macfarlane, Glynn, Grace, Penetito, and Bateman (2008) describe consistencies between the curriculum's key competencies and an indigenous Māori worldview, which they maintain offers a useful pathway towards their understanding and implementation. For example, whakawhanaungatanga (building and maintaining relationships) can be seen within educational settings to include implications regarding how the terms 'knowledge', 'pedagogy', 'discipline', and 'curriculum' can be understood (Smith, 1995). Furthermore, while whakawhanaungatanga can be seen to sit well beside the key competency 'relating to others', it goes beyond a western/European view of establishing and nurturing teacher-student relationships 
(Macfarlane et al., 2008). Examples include that the term implies a greater focus on the responsibility of care for others in its broadest sense and necessitates a blurring of the boundaries between teachers' personal and professional lives.

The curriculum requires that mathematics and statistics be taught in ways that enable students to see their relevance to everyday and other meaningful contexts. This emphasis is shown within the essence statement for the mathematics and statistics learning area (Ministry of Education, 2007a) and the prefacing statement for all of the curriculum's mathematics and statistics achievement objectives:

In a range of meaningful contexts, students will be engaged in thinking
mathematically and statistically. They will solve problems and model situations
that require them to... (Ministry of Education, 2007a, fold-out charts)

The emphasis on real life and meaningful contexts for mathematics and statistics is not new to the current curriculum; however, challenges such as the lack of appropriate resources, and the diversity of students' everyday lives, can make it difficult for teachers to implement this ideal (Averill, 2001).

There are roughly 760000 students across New Zealand's primary and secondary schools of which $59.0 \%$ are New Zealand European, $21.6 \%$ are Māori, and $9.1 \%$ are of Pasifika heritage (Ministry of Education, 2007b). The proportions of Māori and Pasifika students are gradually increasing (showing $6.4 \%$ and $12.8 \%$ increases respectively between 2002 and 2006) while the enrolments of New Zealand European students have dropped slightly, with a 3.6\% decrease between 2002 and 2006 (Ministry of Education, 2007b).

There is a poor match in urban schools between the ethnicities of New Zealand secondary school teachers and those of their students. The proportions of teachers at New Zealand state and state integrated secondary schools with New Zealand European, Māori, and Pasifika ethnicity are 75.2\%, 7.4\%, and 2.2\% respectively while the proportions of students in urban secondary schools with these ethnicities are $48 \%, 19 \%$ and $9.2 \%{ }^{2}$. The ethnicity data for teachers in specific subject areas are not available, nor are data linking teacher ethnicity to school type (e.g., urban, non-urban, state, single sex...) but it is possible that the differences between the proportions of teachers and students of New Zealand European, Māori, and Pasifika

2 Data recorded in July 2007 and supplied by the Data Management and Analysis Division, Ministry of Education, $30^{\text {th }}$ July 2008. 
ethnicities are greater than those quoted above for mathematics teachers and students ${ }^{3}$.

Villegas and Lucas (2002) warn that the differences between the lives of teachers and their students "make it increasingly difficult for teachers to design instruction that capitalizes on the background experiences of their students" (p. xii). Furthermore, they warn that the relative absence of teachers of students' ethnicities within schools deprives students of "role models", "cultural brokers", and "cultural advocates" (p. xii). They and others (e.g., McDonald, 2005; Mills, 2008) are concerned that teacher education has often dealt with cultural diversity issues in a light or piecemeal manner and call for effective ways of preparing teachers to address cultural issues.

Students' cultural heritages and home backgrounds vary greatly both across and within ethnicities. For example, there is growing awareness that Māori are not and cannot be considered one homogeneous group (Ka'ai, Moorfield, Reilly, \& Mosely, 2004; Ministry of Education, 2006b; Pere, 2008). There are differences between customs and language both across and within iwi (Māori tribal affiliations) and great variation exists regarding how strongly people identify with their iwi or Māori roots and with the 'mainstream' (predominant New Zealand) culture. As for Māori, 'Pasifika peoples' is a descriptor that encompasses many diverse groups of people. It includes all those across New Zealand who identify with Pacific Nations (e.g., Samoa, Cook Islands, Tonga, Niue, Tokelau, Fiji, Solomon Islands...) including those of mixed heritages, and those of initial and all subsequent generations of Pasifika peoples in New Zealand (Samu, 2006).

For many Māori and Pasifika students, teacher-student interactions happen within the interface of two "culturally-embedded worlds: the formal education system....and the world of the individual learner" (Samu, 2006, p. 46). The apparent 'cultural discontinuity' between students' homes and school environments has over recent years attracted growing attention both nationally and internationally (e.g., Bishop et al., 2003; Gay, 2000; Gorinski et al., 2008; Nieto, 1999; Nieto \& Bode, 2008) and increasingly attempts are being made to recognise, understand, and reduce its effects on student affect, engagement, and achievement (e.g., Tyler et al., 2008). The greater the understanding of Māori and Pasifika identities, issues, and backgrounds teachers can bring to these interactions and the more they are able to

\footnotetext{
${ }^{3}$ Ethnicity data for New Zealand tertiary mathematics students could not be obtained. The statement is therefore based on personal experiences and informal consultation with tertiary mathematics educators.
} 
create learning communities meaningful for these students, the more effective these interactions are likely to be.

Over ninety percent of Māori students aged between twelve and fifteen attend English-medium education (Ministry of Education, 2007b). High achievement for Māori students in English-medium schools and strong teacher-student relationships are seen as going hand in hand (Bishop et al., 2003; Ministry of Education, 2006a). Being able to develop and maintain effective teacher-student relationships is now the focus of national teacher professional development programmes (e.g., Te Mana Kōrero, Ministry of Education, 2007c). The current focus of the New Zealand Ministry of Education on teacher-student relationships and calls for greater understanding of these (e.g., Trinick, 2006), highlight the timeliness of exploring how teachers can develop and nurture effective teacher-student relationships with Māori students. Given the historical and persistent differences between Māori and New Zealand European students on traditional measures of mathematics achievement (Boustead \& Strathdee, 2008; Clark, 1999; Crooks \& Flockton, 2002; Flockton \& Crooks, 1998; Flockton, Crooks, Smith, \& Smith, 2006; Forbes, Blithe, Clark, \& Robinson, 1990; Ministry of Education, 2006a) such studies are particularly urgent.

The Ministry of Education's Māori education policy Ka Hikitia (Ministry of Education, 2008c) is a call to action for all those involved in New Zealand education (learners, families, professional teachers, hapū (family groups), and iwi). It aims to provide:

...a way for wider educational strategies to be personalised to meet the needs, aspirations and expectations of Māori and the value they expect, want and deserve from the system. (p. 4)

...the opportunity to transform the system's performance for and with Māori, so that Māori are able to enjoy education success as Māori. (p. 4)

The second of four focus areas within the policy targets improving education opportunities for students in Years 9 and 10. The policy builds on the view that acknowledging, reflecting, and valuing student identity and culture are essential for success (Ministry of Education, 2008c). The goals include ensuring effective teaching and learning for and with Māori students, increasing student involvement in and responsibility for education-based decision making, improving whānau-school relationships, and ensuring professional development programmes are evidencebased. 
Ka Hikitia's (Ministry of Education, 2008c) central vision is ensuring Māori students are able to achieve "as Māori" (p. 4). Whilst acknowledging the vision's meaning will vary across individuals, the policy promotes the following towards this vision:

- "knowing, respecting and valuing who students are... and building on what they bring with them" (p. 20);

- providing support for Māori learners within te ao Māori (Māori worldview and values) in order that they have a strong sense of cultural identity;

- adopting the reciprocity of teacher and learner, quality teacher-student relationships, and shared decision-making offered by the concept of 'ako' (defined as both learning and teaching);

- developing partnerships between all involved in education (e.g., learner, teachers, whānau) and adopting a Māori worldview that the learner and their whānau are not separate entities; and

- ensuring Māori learners see themselves in their education, realise their "cultural distinctiveness and potential" (p. 18), participate in and contribute to te ao Māori, and achieve what they need for success in their "lives as Māori" (p. 18).

The policy provides strategic goals and priorities for action to assist with realising Māori students' potential. The priorities for pre- and inservice teacher education highlight actions that underline the importance of nurturing relationships between teachers and Māori students that lead to improved student engagement and achievement, and enable students to be actively involved in their learning.

Often considered New Zealand's founding document, the Treaty of Waitangi guarantees to Māori equal status with Pākehā (non-Māori New Zealanders). Teachers are required to reflect the Treaty principles (partnership, protection, and participation) in their practice (e.g., Ministry of Education, 1998; New Zealand Teachers Council, 2008). Although a national requirement, guidance on how the implications of the Treaty can be reflected in classrooms and practice varies (Averill, Anderson, Easton, \& Hynds, 2004).

The number of students studying predominantly in te reo Māori (the Māori language) is steadily increasing, with a 10\% increase since 2000 (Ministry of Education, 2007d). There is evidence that students studying within Māori immersion settings enjoy higher levels of achievement than their English-medium counterparts (Alton-Lee, 2003; Ministry of Education, 2007d) indicating that learning settings consistent with te ao Māori are more conducive to Māori students' achievement. 
The Education Review Office reviews and reports on each New Zealand school approximately every three years, using a framework of school specific priorities, areas of national interest, and compliance issues (Education Review Office, 2008). Research projects related to Ministry of Education policy implementation and their review work are another focus of the organisation. Sixteen schools that demonstrated quality educational practices for Māori students resulting in improved Māori achievement were the focus of one such study (Education Review Office, 2002). School review reports from the selected schools were analysed and interviews carried out with Māori students, parents, members of the schools' governance, principals, teachers, and other school staff. Findings included that the study schools affirmed success, promoted positive behaviour management strategies, facilitated peer learning support, provided leadership opportunities, and encouraged parental and community involvement. The schools' practices were found not only to benefit Māori students but also to provide high quality teaching and learning overall. The schools incorporated "substantial elements of traditional and contemporary Māori language, culture and knowledge into the curriculum and general operations of the school" (Education Review Office, 2002, p. 1). That only two of the sixteen selected schools were secondary schools may indicate that at secondary school level further complexity exists regarding the influences on Māori students' achievement or such practices may be more challenging to adopt.

Strong mathematics achievement gains by Māori students in English-medium schools have also been linked to characteristics of the school environment, pedagogical practices, and teachers' personal qualities (Te Maro, Higgins, \& Averill, 2008). A shared school commitment to promoting Māori students' achievement and adopting culturally responsive orientations within school and classroom environments were found in schools that attained high Numeracy gains for many Māori students. Specific examples of effective practice included acknowledging and incorporating taonga and tikanga Māori (Māori treasures and practices) such as using the Māori language and nurturing whanaungatanga (relationships within a wider community), making personal connections between learning and the learners, and developing shared responsibility for students' learning (Te Maro et al., 2008).

Traditional Māori pedagogies include experiential learning, context-based and integrated learning, tuakana-teina (learning from more experienced peers), learning through stories, metaphor, and song, and encouraging student responsibility for learning (Hemara, 2000). Common themes from recent research into Māori students' learning in English-medium secondary schools (Bishop \& Berryman, 2006; 
Bishop et al., 2003; Macfarlane, 2004; Tuuta, Bradnam, Hynds, Higgins, \& Broughton, 2004) included:

- the high importance placed by Māori students and their families on educational success;

- the value of incorporating principles from tikanga Māori (Māori practices) and a whole school developmental approach to responsiveness to Māori students;

- many teachers' lack of awareness of Māori culture; and

- the fundamental importance of quality teacher-student interactions and relationships.

The Ministry of Education's (2006c) Pasifika education plan highlights the goals of improving achievement and increasing the effectiveness of teaching of Pasifika students. These goals are particularly vital in mathematics education given that Pasifika students in New Zealand secondary schools consistently achieve poorer results on traditional mathematics achievement measures than students of other ethnic groups (Boustead \& Strathdee, 2008; Clark, 1999; Crooks \& Flockton, 2002; Ferguson, Gorinski, Samu, \& Mara, 2008; Flockton \& Crooks, 1998; Flockton et al., 2006).

The diversity amongst those described as Pasifika people and the diversity of their learning settings (e.g., home, education in language immersion settings, schools, and often, church) would seem to strongly suggest there are no specific pedagogical techniques or sets of teaching strategies which 'best suit' Pasifika learners (Gorinski et al., 2008; Samu, 2006). However, teaching pedagogies reported as 'useful' within a professional development initiative focussing on improving the literacy strategies of Pasifika students (Ministry of Education, 2007e) included: using humour to encourage engagement, "using Pasifika literature as a bridge into literature reflecting others' experiences" (p. 19), telling stories to explain concepts and to locate information in students' lives and experiences, providing one-to-one support, and encouraging students.

A comprehensive literature review written to inform the Ministry of Education on ways to improve teacher capability towards maximising Pasifika student outcomes (Ferguson et al., 2008) highlights the diversity of backgrounds and learning preferences of Pasifika students and the gaps in research (e.g., learning of Pasifika groups other than Samoan) as challenges to inclusive teaching practices. Furthermore, Ferguson et al. (2008) believe these challenges can increase the potential for "tokenism" (p. 23) in attempting to address the educational needs of Pasifika students. 
Recurrent themes in the literature regarding Pasifika students' learning particularly pertinent to this study include:

- the importance of teacher-student relationships and students having a feeling of connectedness with the teacher;

- the usefulness of teachers incorporating students' views about their learning in their teaching decisions; and

- the lack of teacher recognition of Pasifika students' experiences, needs, and skills (Ministry of Education, 2007e).

In a study involving interviews with Samoan parents of students in Auckland schools, Cahill (2006) found that some believed their children's teachers were ignorant, dismissive of, or insensitive to, Samoan cultural traditions. The parents stated that their children's teachers did not always have high expectations of their children, nor did they teach in ways that allowed for difference in prior knowledge or in the pace of learning. Findings included that the study parents wanted their children's teachers:

- to talk with the children about the children's educational goals and how they could achieve these (p. 63);

- to be approachable and provide encouragement when students were finding the work difficult (p. 64);

- to be faithful and follow up on promises (e.g., of academic support) (p. 64); and

- $\quad$ to care for their children as family members (p. 65).

Lack of teacher knowledge about Pasifika students is a barrier to teachers fulfilling the Ministry of Education's goals for Pasifika students' education (Ministry of Education, 2006c). Many researchers of Pasifika education (e.g., Coxon, Anae, Mara, Samu, \& Finau, 2001; Rata, with O’Brien, Murray, Mara, Gray, \& Rawlinson, 2001) believe that in order to develop their understanding of the diversity of Pacific peoples in New Zealand teachers need to learn about:

- the linguistic, cultural, and culturally-linked pedagogical differences between Pasifika groups and between Pasifika groups and schools' and teachers' own cultures;

- the colonial histories of the Pacific Nations from which people have immigrated and the reasons for immigration; and

- the political and economic relationships between Pacific Nations and New Zealand. 
New Zealand European students are also diverse in terms of their cultural heritage. Large numbers of immigrants (from England, Scotland, Ireland, Wales, Holland, Italy, Greece, Australia, Canada, South Africa, and Eastern Europe) have come to New Zealand since the 1800s. New Zealand European students show a wide range of achievement levels in mathematics but as a group tend to achieve well above their Māori and Pasifika peers (Crooks \& Flockton, 2002; Flockton \& Crooks, 1998; Flockton et al., 2006; Tagg \& Thomas, 2008).

The heritage language of many of the New Zealand European students is English either because their families came from an English speaking country or because their families have been in New Zealand for many generations. The schooling in their heritage countries is in most cases fairly similar to New Zealand's. The New Zealand Ministry of Education has invested in research and teacher professional development targeted towards improving teaching and learning for Māori and Pasifika students (e.g., Bishop et al., 2003; Ferguson et al., 2008) but there are no direct comparisons aimed specifically at improving the achievement of New Zealand European students. Most New Zealand European students are in the ethnic majority in their classes and schools; however, those in this study are not.

The proportion of New Zealand children with mixed ethnic heritage is increasing. In $2001,18 \%$ of all New Zealand children under 15 belonged to more than one ethnic group with more than half of these children of both Māori and New Zealand European ethnicity (Ministry for Culture and Heritage, 2008).

The Ministry of Education allocates a socio-economic rating to every New Zealand school which is calculated using a range of indicating factors within the school's surrounding community (e.g., household incomes, occupations, parents' educational achievement, income support payments, and ethnicity) (Ministry of Education, 2008a). The ratings range from decile one (low socio-economic status) to decile ten (high). Māori and Pasifika people are over-represented in low socio-economic groups in New Zealand.

There is clear international and national evidence linking student participation and achievement with socio-economic status (Chapple, Jeffries, \& Walker, 1997; Crooks \& Flockton, 2002; Fergusson, Lloyd, \& Horwood, 1991; Flockton \& Crooks, 1998; Flockton et al., 2006; Lock \& Gibson, 2008; Nash, 2004). Low socio-economic students often have greater emotional needs than others and therefore nurturing, caring, learning environments are particularly important for their engagement and 
success (Sharp \& Jenner, 2006). These results indicate the importance of understanding the characteristics and factors in teacher-student relationships most valued by low socio-economic students.

\subsection{Cultural identity}

Teachers' and students' cultural identities (in terms of their heritage culture), their understanding of one another's heritage cultures, and the place of these cultures within mathematics learning are fundamentally important to this study. This section provides an overview of three areas of the literature: how 'culture' is defined, how students' cultures are reflected in New Zealand classrooms, and culturally responsive practice.

Weiss, Kreider, Lopez, \& Chatman (2005) define culture as "a set of values, norms, beliefs and symbols that define what is acceptable to a given society, are shared by and transmitted across members of that society and dictate behavioural transactions within that society" (p. 137). Matthewson \& Thaman (1998) are among others who add to this definition, suggesting that people of different cultural groups have different ways of perceiving and organising the world around them. Culture is usually linked to ethnic heritage and is widely accepted to be dynamic and constantly evolving in response to social and physical structures and family and school processes (Mara, 1998).

Blum (1999) defines four types of ethnicity, two of which are a matter of circumstance ('thick' and 'thin') and two of which are self-chosen ('identity' and 'antidiscriminatory'). 'Thick ethnicity' is characterised by people living in largely monoethnic neighbourhoods and whose family life is permeated by ethnicity-based rituals and cultural expressions of ethnicity. Those with thick ethnicity are able to speak the language of their ancestors, and have friends almost exclusively of their own ethnic group. For some cultural groups they tend to live in poorer communities, and may have more recently immigrated.

Those fitting Blum's (1999) definition of having 'thin ethnicity' take part in some ethnicity-linked cultural activities but tend not to live primarily with those of their own ethnic group. Ethnicity is not very important to their daily existence. They can only speak the language of the majority, are more distant from their immigrant ascendants, and are generally more financially secure than the poorer 'thick' communities. 
Blum (1999) refers to a third group as having 'identity ethnicity.' This group is greatly assimilated into the dominant culture but physically and visually they are of a particular ethnicity and for this reason tend to identify with, or be identified with, that ethnicity. The degree to which they identify with the ethnicity varies. This group has social identity but generally lacks knowledge of the cultural practices associated with their heritage ethnicity. Blum's final group is those he describes as having 'antidiscriminatory ethnicity.' This type is grounded in individuals' feelings that the ethnic group is a target for discrimination. People in this group may not identify themselves as culturally ethnic but identify with the ethnicity of a group that is discriminated against.

The four types in Blum's (1999) model are not necessarily distinct; people can move between groups and differing values and beliefs exist within each group. However, the ethnicity types can provide a useful tool to help understand culturally diverse contexts and they afford an indication of the complexity of addressing the learning desires and needs of culturally diverse classes.

Further layers of complexity exist within New Zealand contexts. New Zealand classrooms are becoming increasingly culturally diverse with:

- $\quad$ increasing numbers of ethnicities represented in New Zealand society;

- increasing proportions of students of some ethnic groups (e.g., Māori and Pasifika); and

- increasing numbers of students of mixed ethnic heritage.

Each person's own culture is often so habitual it is invisible to them; it is often easier to notice differences between others and themselves than aspects of their own culture (Banks, 2004; Nieto \& Bode, 2008; Pang, 2005). This is particularly the case for people with heritage closely comparable to society's dominant culture, such as New Zealand European students.

A wide range of attitudes and beliefs about the personal relevance of individuals' ethnicity exists and hence students' ethnic identities may or may not be the same as their cultural identities. For example, a student's ethnicity may include both Māori and New Zealand European heritage but their cultural identity, by choice or by nurture, may include elements consistent with New Zealand European culture or Māori culture or both to varying degrees. Despite the complexity of issues surrounding the cultural identities of New Zealand students, many Ministry of 
Education research and policy documents (Bishop et al., 2003; Ferguson et al., 2008; Ministry of Education, 2006c, 2008c) call for pedagogy that acknowledges and validates students' individual and collective diversities and which enable students to see themselves and their cultures reflected in their classrooms. Although much is written about the link between cultural identity and academic achievement internationally (e.g., Arnaiz, Berruezo, de Haro, \& Martinez, 2005; Ogbu, 1992), few studies exist which explore the interplay between New Zealand students' cultural identities and their learning, particularly in mathematics.

There is some evidence that many Pasifika students readily self-identify with their culture (e.g., Tupuola, 1998); however, many school students are keen to downplay or hide manifestations of their heritage culture (Sheets, 2005) and may avoid acknowledging their ethnic ancestry. Evidence that environmental factors in New Zealand schools affect such decisions is indicated by a twenty percent increase in students identifying as Māori in one school, where "students recognised that they were valued as Māori” (Gorinski \& Shortland-Nuku, 2006, p. 17).

An exploratory study of effective practice (curriculum, pedagogy, and assessment) for Pasifika students (Gorinski et al., 2008) in eight New Zealand schools found diversity in students' views and understandings about their personal and cultural identities, and their identities as Pasifika learners. The report emphasises that because Pasifika identities are not static and many students identify themselves in multiple ways, teachers need to develop classroom relationships that allow them to know and cater well for their students as individuals. Data from focus group interviews held with teachers as part of their research indicated that teachers "had varied understandings of the relevance of culture in teaching" (p. 74). Gorinski et al. call for professional development focused on improving teachers' cultural knowledge as an essential step towards improving pedagogical practice for Pasifika students.

Nakhid (2003) hypothesised that students being able to engage with an identifying process, with teacher recognition, is necessary for academic success. However, Nakhid found few opportunities available in schools for Pasifika students to develop their own identities. Nakhid's interviews with five mathematics teachers (four New Zealand European and one New Zealand Chinese) found that despite all having at least seven years experience teaching Pasifika students, they knew very little about their Pasifika students' identities, about Pasifika parents, or the role the parents play in their children's education. 
As a result of her findings from a study of New Zealand Tongan students in Auckland secondary schools, Manu'atu (2000) advocated linking Tongan worldviews, cultural practices, and language into the school curriculum. She described two examples of how acknowledgement, understanding, and integration of elements of Tongan culture can assist in raising the achievement of these students. Manu'atu described the Tongan concept of mālie as the uplifting, energising atmosphere felt by performers and their audience through a sense of connection between and commitment to acts of performance, communication, and the human psyche and spirit, and suggested that for New Zealand Tongan students to be successful, the learning process must hold a sense of mālie. Manu'atu also found teacher-student warmth to be important for student learning.

Further evidence of the importance for students' learning of making Pasifika values, cultural knowledge, and language identities implicit in teaching and learning practices is provided by Fletcher, Parkhill, Fa'afoi, Taleni, and O'Regan's (2009) study of factors affecting the achievement of New Zealand Pasifika students in Years 5 to 9. This study built on the authors' previous work (e.g., Parkhill, Fletcher, \& Fa'afoi, 2005) exploring teachers' and parents' perceptions of the supports and barriers to literacy and other achievement of Pasifika students. They conclude that valuing students' cultural capital through acknowledgement, making connections between the students' first language and the language of instruction, and fostering home-school links are all important for maximising achievement of Pasifika students.

A study into how teachers in five culturally diverse New Zealand city schools manage cultural diversity (Loorparg, Tait, Yates, \& Meyer, 2006) found that most teachers attempting to use culturally responsive techniques used primarily 'contributions' and 'additive' approaches (Banks, 2004) to raise multicultural awareness. 'Transformational' and 'social action' style pedagogies (Banks, 2004) which more deeply integrate multicultural themes were seldom found. The report highlighted that many New Zealand teachers feel that adopting culturally responsive practices tends to be left for individual teachers to tackle. Teachers called for greater support in a range of areas to cater more effectively for the cultural diversity of their classes (e.g., inter and intra-school professional development, provision of resources addressing cultural diversity, greater numbers of specialist teachers from whom they could ask advice and seek support, and improved links between schools and communities). Loorparg et al.'s (2006) study explored the breadth of multicultural practice used to address the cultural diversity of secondary classrooms generally and the findings were not stated in relation to specific cultural groups or to specific subject areas. 
There is a growing body of research and other writing internationally and nationally in the area of culturally responsive practice. Gay (2000), Ladson-Billings (1990), Villegas and Lucas (2002), and Wlodkowski \& Ginsberg (1995) all provide useful models for culturally relevant and culturally responsive teaching and/or teacher education (Chapter Two). In keeping with New Zealand's bicultural partnership, ${ }^{4}$ New Zealand literature includes Māori-centred and bicultural approaches to inform teachers' culturally responsive practice (e.g., Averill, Anderson et al., 2009; Averill, Te Maro, Taiwhati, \& Anderson, 2009; Bishop, 2008; Macfarlane, 1997, 2004; Pere, 1994, 1997).

Ferguson et al. (2008) draw from a wide literature base (including Alton-Lee, 2003; Banks, 1994; CREDE, 2004; Gay, 2000; and Sheets, 2005) to recommend seven key dimensions for culturally responsive teaching. 'Inter-personal relationships' are the focus of one of the seven dimensions. To support teachers' understanding of this dimension, the authors make a strong call for "school-based, in-depth research that explores and analyses Pasifika students and their personalised interactions and relationships with their teachers, particularly in terms of how such relationships affect their academic engagement and success" (p. 32).

The New Zealand curriculum (Ministry of Education, 2007a) and a landmark New Zealand report synthesising the best evidence from many New Zealand and international studies on effective pedagogy for mathematics (Anthony \& Walshaw, 2007) call for practice that enhances students' sense of cultural identity. Studies that explore the current state of culturally responsive practice in New Zealand mathematics classrooms, particularly in terms of Māori and Pasifika students and teacher-student relationships, are clearly needed. This is an important area to investigate given the increasing proportions of students with these cultural heritages in New Zealand schools and the persistent achievement differences between these students and others.

In understanding how cultural similarities and differences may affect our classrooms, it can be helpful to consider cultural behaviours and understandings such as: how emotion, interest, and respect are displayed; when questioning is appropriate; how lateness is interpreted; how conversations are begun (e.g., by re-establishing personal connections before getting into core business); and how body language is used and interpreted. These and other characteristics of person-to-person interactions can vary from one culture to another, are easy to misinterpret, and misinterpretation can undermine the quality of inter-personal relationships.

4 The bicultural partnership was established formally between Māori and the British crown by the signing of the Treaty of Waitangi in 1840. 


\subsection{Classroom learning}

This section briefly introduces theoretical perspectives that support the notion that quality teacher-student relationships are important for maximising students' learning. Student engagement, motivation, and achievement, the classroom environment, adolescent development, learning of mathematics, and affect and achievement are discussed in turn in relation to the focus of this study.

There is a growing body of evidence regarding the influence of effective teacherstudent relationships on student engagement, motivation, and achievement. Teacher-student relationships are strongly related across ethnicities to sustaining student engagement, achievement, and future education (Ferguson, 2002; Holloway, 2004; Hudley \& Daoud, 2007; Meece \& Daniels, 2008; Mortimore, Sammons, Stoll, Lewis, \& Ecob, 1988; Thomas, Smees, MacBeath, Robertson, \& Boyd, 2000). Teacher supportiveness (i.e., nurturing, respectful attitudes conveying personal interest and expectations) as perceived by students, is an important factor in students' perceptions of the wider school climate (Hudley \& Daoud, 2007) and is likely to be particularly important for motivation for the lowest-achieving students (Hudley \& Daoud, 2007; Midgley, Feldhauer, \& Eccles, 1989). Teacher encouragement, help, understanding, and friendliness have also been found to be important for student motivation and achievement (Ferguson, 2002; Fisher \& Rickards, 1996; Fisher, Waldrip, \& Chuarch, 2003) supporting the importance of the role of effective teacher-student relationships in promoting achievement.

It is widely accepted that the classroom context also greatly influences students' motivation and achievement (Anthony \& Walshaw, 2007; Eccles \& Wigfield, 2002). These effects are known to vary across students from different cultural backgrounds and are likely to be further confounded by differences across cultures in how 'achievement' is perceived both in terms of what constitutes achievement and its relevance to individuals. Students of some cultures can be more influenced by extrinsic motivators (e.g., testing) and of other cultures more influenced by intrinsic motivators (e.g., personal relevance of contexts of the learning experiences) (Salili \& Hoosain, 2007).

Students from different cultural backgrounds have also been found to interpret similar teacher actions in different ways. For example what can be interpreted by some as teachers' apparent lack of engagement with individuals and hence lack of personal interest in them, can be seen by others as teachers being focused on learning and caring for achievement (Hudley \& Daoud, 2007). 
Boaler \& Greeno (2000) argue that the nature of mathematics learning environments influences students' decisions regarding whether or not they will continue with mathematics learning. They suggest that students in environments that encourage the development of agency and personal identity, in which personal relationships are integral to learning, and in which they are co-authors of their mathematical understanding are those most likely to pursue further mathematics study. Similarly, Boaler (2004) found classrooms that were particularly effective for American minority students' mathematics learning included those in which teaching practices emphasised the development of students' sense of responsibility for one another's learning and in which public specific acknowledgement was linked to learning. Others also regard sharing ownership of learning with students as important (e.g., Wlodkowski \& Ginsberg, 1995).

Year 10 students are in early adolescence. While there is some evidence that adolescence is experienced differently across cultural groups (e.g., Tupuola, 1998), at this stage of their lives, the personal identities of many students are beginning to take shape. This includes the development of their personal thoughts, morality, and ethics (Jackson \& Davis, 2000; Meece \& Daniels, 2008). They are involved in decisionmaking and boundary testing as they are striving to become themselves. Those in early adolescence are moving from concrete-operational thinking to more abstract, hypothetical, and future-oriented thinking (Stoll et al., 2003) and it is an important time for developing positive social relationships (Eccles \& Midgley, 1989). Their developing understandings, beliefs, and perspectives affect adolescents' perceptions of interpersonal relationships and classroom environments (Boaler \& Greeno, 2000; Connolly, Hatchette, \& McMaster, 1998). Nurturing, supportive learning environments that encourage student ownership and incorporate meaningful learning experiences are particularly important during this developmental stage (Eccles, 2004; Perso, 2004; Stoll et al., 2003).

Differences between mathematical learning and learning in general may also impact on the characteristics of teacher-student relationships within mathematics classrooms. Mathematical thinking is considered to be different to thinking in other curriculum areas particularly in terms of problem solving methods, mental flexibility (the ability to move between ideas and different representations of these ideas, e.g., equations, diagrams, words), the ability to perceive and continue patterns, and the propensity to generalise (Watson, 2002). Mathematics students need to be interested in and able to use and persevere with these ways of thinking in mathematical and real life contexts even while encountering obstacles such as difficult content (Watson, 2002). 
Mathematics teachers need to be particularly attuned to these factors and are likely to need to place greater emphasis on specific acts of care (such as use of encouragement, building mathematical confidence, and ensuring students perceive the work to be personally relevant) than teachers in other curriculum areas.

Many studies show links between students' affective views and mathematical achievement. Examples include links between affect and: student attitude (e.g., Antonnen, 1969); students' perceived confidence (e.g., Bouchey \& Harter, 2005); and the perceived usefulness of mathematical achievement (e.g., Fennema \& Sherman, 1978). In the New Zealand context, Nuthall (1999) describes five studies in science and social studies classrooms that found similarly significant effects on learning due to the classrooms' social and cultural factors to those of the intellectual climate of the classroom. Nuthall calls for greater understanding regarding the links between the social experience of the classroom and cognition and calls for teachers to learn more about the social and cultural characteristics of their students in order to cater more effectively for students' individual needs.

\subsection{The study focus}

The literature in the areas described above clearly identifies a need for further investigation of effective teacher-student relationships to facilitate movement towards greater equity in achievement in education and to assist with the implementation of a new curriculum more overtly focused on inter-personal skills. Therefore, within the context of teacher care, this study explores the characteristics of caring teacher-student relationships and the factors that help to establish and maintain such relationships for Māori, Pasifika, and New Zealand European students in urban, low decile mathematics classrooms.

Teacher care is considered by many to be the foundation for effective teacherstudent relationships (e.g., Elias et al., 1997; Powell, 2000). Teacher care enables students to feel important, accepted, and respected and thus they are more likely to express themselves freely and take risks in their learning because they feel safe and that they will be affirmed. Bishop et al. (2003) highlight aspects of teacher care within their "effective teaching profile" (pp. 95-116): "caring for the person" (p. 96), "caring for performance" (p. 99), and "caring for students as culturally located human beings" (p. 96). In order to understand teacher-student relationships, how teachers exhibit these areas of care was chosen as the focus of this study. 
Within the context of Year 10 mathematics classrooms, three aspects of teacher care were investigated: (i) teacher care for students as individuals; (ii) teacher care for students' mathematical progress; and (iii) teacher care for students as culturally located individuals. Questions of particular interest included:

- What are teachers' and students' views regarding the importance of teacherstudent relationships and how teacher-student relationships are created and nurtured? (Chapters Six and Seven)

- Which teaching pedagogies are particularly effective for developing caring teacher-student relationships? (Chapters Five and Nine)

- Do learning experiences grounded within contexts meaningful to the students assist in developing caring teacher-student relationships? (Chapters FiveNine)

- What similarities and differences exist between students of different heritage cultural groups regarding caring teacher-student relationships? (Chapters Seven and Eight)

Because research suggests low socio-economic status students are both less likely to achieve academically and more likely to need caring learning environments, in keeping with the study theme of exploring ways towards greater equity in educational achievement, low-socio-economic schools were selected as the target group for this study. Similarly, as previously discussed, evidence shows Māori and Pasifika students have traditionally not been well served in New Zealand educational contexts and effective teacher-student relationships are important for their academic success. Thus the target group for the study includes low socioeconomic schools with approximately $30 \%$ each of Māori and Pasifika students.

Year 10 classrooms were chosen for this study: to match Bishop et al.'s (2003) study focus on Year 10 and to reduce the potential for study results to be confounded by any effects of formal external assessment (Years 11-13) or by students adjusting to a new environment and establishing new relationships with peers (Year 9). Furthermore, formal success in mathematics is known to be a gatekeeper (Barton, 1995) for later educational choices and career pathways. Students' experience of mathematical success in Year 10 is likely to influence their decisionmaking regarding subject choice for qualification-based assessment, which for most students, begins in Year 11. Evidence exists that minority students drop mathematics earlier than others (Holloway, 2004). As Year 10 immediately precedes 
assessment for qualifications the study may therefore shed light on effective practice for enhancing students' formal mathematics achievement and retaining students in mathematics study.

Classes of able and average to above average Year 10 students were selected as the target group for the study as these students were deemed those most likely to still be engaged with mathematics learning and to continue with some success into further mathematics study. Finding what works for these students regarding teacher-student relationships is important to maximise their continued participation in mathematics. Lack of mathematical success, negative feelings towards mathematics, and lack of hope for personal mathematics achievement, all of which can reduce motivation for learning mathematics, were thought likely to be comparatively prevalent for less able low socio-economic Year 10 students, making these students less suitable for this study.

Research-based evidence regarding the nature of caring classroom relationships and the importance of such relationships for New Zealand students will inform preand inservice teacher education, education policy, and international debate regarding reducing disparity in mathematical achievement.

A socio-cultural paradigm frames this study (Chapter Three) and it is acknowledged that the researcher's interpretations of the study contexts, interactions, processes, and data are shaped by the researcher's own lived experiences (Creswell, 2003). Salient components of the researcher's background are briefly described below to allow interpretation of the data analysis and study findings in the light of the researcher's prior experiences.

Aspects of personal experience most relevant to this study include my own education, where I have lived, the way in which I was brought up, and my family situation. My first years of schooling were in Memphis, Tenessee in the 1960s. In my memory, race was a noticeable and divisive aspect of society: African American people tended to hold jobs such as picking cotton, garbage collecting, and serving others (e.g., maid). Across the two predominantly 'white' schools I attended, there was only one African American teacher. Martin Luther King was assassinated in Memphis while we lived there. The rest of my schooling and childhood was in New Zealand and included attending one private and several state co-educational and single sex schools. My teaching background includes teaching at two large coeducational city secondary schools, and pre- and inservice teacher education. My 
family had a keen ethical sense with my grandfather and great grandfather working within the Anglican Church. At the time of this study my son was roughly the same age as the student participants.

My interest in the study topic arises through my long-held commitment to exploring ways to achieve greater equity in compulsory school sector mathematics participation and achievement, particularly for Māori and Pasifika students. My previous research includes explorations into culturally responsive mathematics education practice, particularly using bicultural frameworks, and the production of equity-oriented mathematics teaching resources (e.g., Averill, Anderson et al., 2009; Averill, Phillips, \& French, 2003; Averill, Te Maro et al., 2009; Mclntyre, 1994). All of my educational experiences as a learner and a teacher in schools and teacher education, and my interactions and relationships with students, mathematics teachers, and colleagues have helped to inform my research and my teaching philosophy and practice.

Early in the study it was decided that cultural advice would be sought throughout to help ensure responsiveness to Māori and Pasifika cultural nuances and behaviours. Cultural advisors amenable to continued involvement were secured, one Māori and one Pasifika. Their selection and roles are discussed in Section 3.2.

\subsection{Overview of chapters}

The thesis describes the rationale for and context of the study, uses pertinent literature to frame the research, explains how the study was carried out, presents analysis of the data, and discusses conclusions and implications of the study findings.

In particular:

- Chapter Two describes recent studies and the current literature and knowledge about teacher care used to inform the study method, data gathering tools, and data analysis.

- Chapter Three outlines and justifies the methodology and methods of the study, the development of data gathering tools (observation schedules, interviews, and questionnaires), the selection of the study participants, the analysis methods, and the model developed to describe the study findings.

- Chapter Four provides contextual information relevant to the study participants; the study schools, teachers, and classes. 
- Chapter Five focuses on classroom practices that help create caring teacherstudent relationships using analysis and findings from the data gathered through classroom observations and audio-recordings.

- Chapter Six describes the study teachers' perspectives on teacher care determined primarily through the analysis of data from teacher questionnaires and interviews.

- Chapters Seven and Eight present students' views about teacher care again determined through the analysis of data from questionnaires and interviews, this time using students' data.

- Chapter Nine summarises themes that emerged frequently throughout the study and thesis and summarises the study findings in relation to the main model used for analysis.

Finally, the overall study conclusions and links between the study findings, literature, and Ministry of Education policy documents are given in Chapter Ten. Issues affecting the research process and results are discussed and suggestions for future areas of research outlined. 


\title{
CHAPTER TWO
}

\section{Teacher Care: The Literature}

\begin{abstract}
Caring is central to the shaping of relationships that are meaningful, supportive, rewarding, and productive. Caring happens when children sense that the adults in their lives think they are important and when they understand that they will be accepted and respected, regardless of any particular talents they have. Caring is a product of a community that deems all of its members to be important, believes everyone has something to contribute, and acknowledges that everyone counts. (Elias et al., 1997, p. 6)
\end{abstract}

The literature provides a strong case for teachers to show care to their students and provides a broad range of teacher behaviours known to be and advocated as 'caring'. However, much of this literature is neither based within New Zealand contexts (and therefore may not be directly applicable to our unique cultural setting) nor specifically focused on the interplay between teacher care and mathematics learning. Many aspects of caring teacher-student relationships appear common across varied learning environments. Further research is needed to illuminate key similarities and differences between how teacher care is expressed and interpreted within specific subject areas.

This chapter provides an overview of the literature regarding teacher care by: introducing the main studies and other key literature used to inform this project (Section 2.1); examining why teacher care is important to explore (Section 2.2); and describing the main aspects of teacher care that relate to this study (Sections 2.3 and 2.4).

The factors that help constitute a 'caring relationship' can vary across ethnicities (Thompson, 1998). Furthermore, teachers and students of different cultures can easily misinterpret one another's actions and interactions (Clark, 2001; Greenbaum \& Greenbaum, 1983). This chapter presents findings and views from a wide range of literature, some drawn from culturally diverse contexts, and some from comparatively homogeneous groups. It is unlikely that all aspects throughout the chapter discussed as 'caring' practices are experienced as such by all students. In order to capture all possible caring aspects for consideration in this study, all found in the literature are presented here and all were used to inform the development of the study's data gathering tools (Chapter Three).

Some aspects discussed within the chapter appear more than once due to their relevance to the particular aspects under discussion. 


\title{
2.1 Overview of key literature on teacher care
}

The key literature used to inform this study included both empirical studies and landmark authoritative texts. The empirical New Zealand and international studies that have informed this project (Appendix 1) include two valuable New Zealand studies: Bishop et al. (2003) and Hill \& Hawk (2000). Both studies were focused within secondary schools and investigated practice across all curriculum areas.

Bishop et al. (2003) held group and individual interviews of 70 students, 80 teachers, 4 principals, and 50 parents across four urban and rural town Englishmedium schools. The study found effective teacher-student relationships to be vital for the academic success of Māori students at Years 9 and 10:

\begin{abstract}
What is clearly of major importance to Māori students' educational achievement is the quality of the in-class face-to-face relationships and interactions between themselves as Māori people and their teachers. (p. 206)

The Ministry of Education... in its tender for this research identified a number of influences that are reported in the literature that impact upon Māori students' achievement - these included: Home and school relationships, Pedagogy, Teachers' expectations, Schools (climate, environment and leadership), Peer effects, Classroom/group dynamics, Transition (from intermediate or full primary to secondary), Mentors, Whānau support, Socioeconomic factors.

The present study identified that while all of these factors could well influence the achievement of Māori students, most of them were subsumed by the quality of the face-to-face, in-class relationships and interactions between the teachers and Māori students as major influences on Māori students' educational achievement. (p. 192)
\end{abstract}

Bishop et al.'s (2003) study was not intended to investigate the importance of such relationships for students other than Māori, or to include specific focus on mathematics teaching and learning. Their findings indicate clear pathways towards improving academic success of Māori students including a teaching model consistent with excellent teaching practices (Hattie, 2009) ${ }^{5}$. As the findings have been used to inform professional development projects in schools with students other than Māori, it is important to extend aspects of Bishop et al.'s (2003) investigation to these groups.

Hill and Hawk's (2000) study was carried out in eight low decile secondary schools with high proportions of Pasifika students. Over 100 lessons were observed with each followed by an interview with the observed teacher and another with a group of students from the class.

5 It should be noted that critiques of Bishop et al.'s (2003) findings exist (e.g., Openshaw, 2007). 
Both Alton-Lee's (2003) and Anthony \& Walshaw's (2007) comprehensive literature reviews of key messages from national and international literature for teaching and learning mathematics in New Zealand early childhood to secondary school settings identify caring learning communities and in particular 'the ethic of care' as important for effective learning and were used to inform this study.

Other literature used to inform this research includes a range of writing on teacher care, teacher-student relationships, and culturally responsive teaching (Appendix 2). In particular, Nel Noddings has written widely about teacher care, caring classrooms, and caring schools (e.g., Noddings, 1992; 1993; 1995; 2003). Other key texts included Elias et al. (1997), Haynes, Ben-Avie, and Ensign (2003), McCombs and Whisler (1997), all of which draw from a range of research and theoretical literature. Gay (2000) pulls together key findings and thinking of many researchers and theorists to give clear messages about culturally responsive teaching. Similarly Good and Brophy (2003) summarise salient information from an extensive range of sources regarding teacher-student relationships and researching classrooms.

\subsection{The importance of teacher care}

\subsubsection{Modelling and teaching an important human value}

People all need to feel cared for. Elias et al. (1997) and McCombs and Whisler (1997) suggest that we work better when we are part of a caring community. Manaakitanga (nurturing relationships, looking after people, and being careful how others are treated) is the foundation for all tikanga (Māori cultural practices) and is important for Māori no matter the circumstances (Macfarlane et al., 2008; Mead, 2003). Traditionally, high value is placed by Māori on manaakitanga and host groups go to great lengths to ensure their responsibility of manaakitanga is met. There are implications of such beliefs for New Zealand classrooms; students and families rich in tikanga Māori are likely to hold this view of caring behaviour and may expect consistency with these views in teacher practice. Those who do not experience such care may feel offence and alienation.

Care for others is similarly important to Pasifika groups. As an example of Pasifika perspectives, Tongan cultural and social values such as "'ofa (love), faka'apa'apa (respect), fetokoni'aki (sharing) and foaki (giving)" govern individuals' behaviours (Koloto, 2004, p. 62). Children are seen as gifts, blessings, and treasures from God; "the future of the family, church and nation" (p. 64). Care for children is seen as very important and is a shared responsibility across the extended family. The main 
purpose in life is the holistic development of three aspects of the "tangata kakato" (the total person) (p. 61): "mo'ui fakasino" (physical well-being), “mo'ui faka'atamai” (intellectual well-being), and "mo'ui fakalaumalie" (spiritual well-being) (p. 62) illustrating the high level of importance in Tongan society of holistic care for others.

Peterson (1992) discusses the importance for their learning of students feeling they are part of a caring community, claiming "community in itself is more important to learning than any method or technique. When community exists, learning is strengthened" (p. 2). He goes on to state his belief that the care and interest of others "breathes purpose and life into learning. Learning is social" (p. 3). Gay (2000) agrees, claiming "teachers who genuinely care about students generate higher levels of all kinds of success than those who do not" (p. 47).

Hawk and Hill (2000) refer to learning as a complex process that necessitates addressing social as well as educational and administrative domains. Similarly, Noddings (1992) claims "schools cannot accomplish their academic goals without attending to the fundamental needs of students for continuity and care" (p. 36). The importance of establishing a positive sense of community within the wider school context is shared by many other authors who hold a common view that an emotional attachment between students and their teachers, peers, and school is crucial for academic success (e.g., Hawkins, Catalano, \& Associates, 1992; Solomon, Watson, Battistich, Schaps, \& Delucchi, 1992 as cited by Elias et al., 1997, p. 45).

Noddings (1992) believes that ways of caring for one another should be explicitly taught. She encourages the view that learning to live with others in the world, rather than improving achievement, should be the primary purpose for teaching care. Gay (2000) strongly advocates caring teacher-student relationships for effective learning and community cohesion, stating:

Caring is a value and moral imperative that moves "self determination into social responsibility and uses knowledge and strategic thinking to decide how to act in the best interests of others. Caring binds individuals to their society, to their communities, and to each other" (Webb, Wilson, Corbett, \& Mordecai, 1993, pp. 33-34). (Gay, 2000, p. 45)

\subsubsection{Impact of teacher care on learning and achievement}

In describing expert teachers, Hattie (2003) highlights the role of teachers' care of and commitment to students in showing high respect for them as learners and as people. Anthony and Walshaw (2007) found much research evidence indicating that an 'ethic of care' (including relationship and confidence building, valuing students' contributions, 
and teacher care for progress in students' mathematical proficiency) is essential for diverse learners' mathematics learning. They describe effective classrooms as those that engender a sense of belonging and mathematical purpose.

Haynes et al. (2003) explored the effect of students' social and emotional development within learning in mathematics and science. They report on a series of experiments that gathered evidence of the impact of interventions on students' social and emotional competence and associated links with mathematics and science performance. They found strong evidence of the importance of teacherstudent relationships for learning mathematics:

The students' relationship with the teacher is key. Much of the learning process involves social interaction. Students are motivated to learn (1) from teachers they respect and feel comfortable with, and (2) from competent teachers who effectively communicate their knowledge and enthusiasm about their subject matter. (p. $x)$

Haynes et al. (2003) discuss how addressing students' social and emotional needs can help enhance both achievement motivation and academic outcomes. Principles that describe the links they found between social, emotional, and academic learning include:

Cognitive development is inextricably linked to social and emotional development.

Social and emotional learning facilitates academic learning and vice versa.

Social, emotional, and contextual factors contribute significantly to children's learning and achievement in math and science.

School climate and classroom climate contribute significantly to the development of positive attitudes and behaviors that result in strong mathematics learning and high mathematics achievement.

Effective math and science teaching and learning involve approaches that integrate strategies that help students make meaning of the processes and content of math and science by building bridges between the content of what is being taught and the life experiences of the students who are being taught. (pp. xiii, xiv)

Hattie's landmark synthesis (2009) of over 800 meta-analyses relating to student achievement highlights the crucial role of teacher-student relationships and emphasises that "developing relationships requires skill by the teacher - such as the skills of listening, empathy, caring, and having positive regard for others" (p. 118). Many other authors also believe that caring teacher-student relationships lead to effective mathematics learning (e.g., Borton, Preston, \& Bippert, 1996; Elias et al., 1997; Gay, 2000; Hill \& Hawk, 2000; Hudley \& Daoud, 2007; McGee \& Penlington, 2001; Noblit, Rogers, \& McCadden, 1995; Noddings, 1992; Peterson, 1992; Powell, 2000). Furthermore, many studies into teacher care are centred on teachers identified by their colleagues, principals, communities, or by researchers as excellent teachers (e.g., 
Mayer \& Marland, 1997; Ramsay \& Oliver, 1995), indicating an implicit assumption that excellence in teaching is inter-woven with caring teacher-student relationships.

\subsubsection{Impact of teacher care on affect and engagement}

Students who believe their teachers are caring and fair are more likely to have positive attitudes to learning, greater motivation to achieve, and increased engagement in learning tasks (Babad, 1996; Hudley \& Daoud, 2007; Wigfield, Eccles, Maclver, Reuman, \& Midgley, 1991; Wigfield \& Harold, 1992). A longitudinal study of 248 students from Year 6 to Year 8 in the U.S.A. (Wentzel, 1997) found that students described effective teachers as having caring attitudes, shown by democratic interaction styles, individualised expectations and constructive feedback, and modelling a caring attitude towards their work. These behaviours helped the students feel valued and motivated to achieve social and academic outcomes (Wentzel, 1997).

Teven and McCroskey's (1996) study included data from 235 students in Communication classes in one North American university. Similar to Wentzel's (1997) findings, they found that students' perceptions of teacher care were closely associated with students' evaluations of the teachers, their dispositions towards the course content, and their perceptions of their cognitive learning. Teven and McCroskey (1996) postulate that it is not the teacher care itself that counts; rather it is the behaviour which leads to students' perceptions of teacher care. They believe that teacher care will not be effective unless it is communicated to students in some way. Teven and McCroskey call for studies that will help identify specific teacher behaviours perceived by students to be caring.

Noblit et al. (1995) report on studies with two teachers (of Year 2 and Year 4 classes) and the positive effects caring relationships had on individual students' academic achievement and social skills. The teachers in the study believed that without relationships with the teacher, students had little reason to commit to instructional activities. One student in their study said:

If a teacher doesn't care about you, it affects your mind. You feel like you're nobody, and it makes you want to drop out of school (Noblit et al., 1995, p. 683).

Students' perceptions of teacher care are also known to enhance their feelings of self-esteem, school belonging, and positive affect to learning (Roeser \& Eccles, 1998; Roeser, Midgely, \& Urdan, 1996). Ocean (2005) interviewed twelve New Zealand women who had become disengaged with mathematical study during their time at secondary school. Ocean explored the effects of the absence of teacher care 
in their mathematics learning and found that feeling uncared for contributed to students' decisions to discontinue with mathematical study. Given the very small numbers of New Zealand mathematics and statistics teachers and academics who have Māori and/or Pasifika heritage, understanding the characteristics of teacher care most valued by these students is vital in endeavouring to ensure these students continue with mathematical and statistical study and potentially into careers that use mathematics and statistics.

\subsection{Students' sense of being cared for at school}

Caring can be expressed in many ways: spoken, enacted, and implied. It can involve showing respect, taking time for informal interactions, giving expert advice, showing affection, or simply giving recognition to a person or their feelings (Noddings, 1992). Expression of care can be part of every interaction and thus teacher-student caring can occur in or outside the classroom and inside or outside the school. People, and in particular students, are attuned to caring. We know when we feel cared for and when we do not. Elias et al. (1997) suggest that the students most attuned to teacher care are the ones who need it the most, the emotionally troubled students.

The literature provides a wide range of ideas regarding how teachers can show care for students. Noblit et al. (1995), however, remind us that care is individualised and is expressed and received by each person in different ways. With this in mind this section describes common features found in caring schools, classrooms, and teacher behaviours, accepting that each teacher will bring together their own unique combination of such features to their classroom interactions with each different class they teach as suits their purpose and style. The section finishes with indicators that may suggest students feel cared for in the classroom.

\subsubsection{Caring school environments}

Many authors discuss the importance of a positive school climate as a necessary pre-requisite for a caring classroom environment (Elias et al., 1997; Haynes et al., 2003; McCombs \& Whisler, 1997; Noblit et al., 1995; Noddings, 1992). Bosworth (1995) and Peterson (1992) offer brief commentaries on traditional school practices, Bosworth claiming that many schools maintain a predominance of uncaring practices that emphasise ways to deal with negative behaviours. Peterson (1992) agrees, citing traditional classroom practices of restricting normal human behaviours such as movement, talking, passing notes, and of establishing classroom control with a 'don't smile until Easter'-type approach. 
Noblit et al. (1995) insist that caring relationships have not been a priority of policy or curricula, and suggest that the "more technical aspects of teaching dominate our thinking about what makes schools and classrooms "good"” (p. 681). They offer specific examples to support this (e.g., schools' competitive approach, traditional discipline approaches, class sizes, daily schedules) and warn teachers to be aware of school and classroom rituals that may undermine a caring atmosphere.

The idea of students caring for themselves and others is consistent with the "key competency', "relating to others" (Ministry of Education, 2007a, p. 12). The five interconnected key competencies are intended as a way of viewing and delivering the curriculum and 'reframing' the essential skills and attitudes of the previous New Zealand Curriculum Framework policy implementation document (Ministry of Education, 1993b). To achieve the goals of the key competencies time and focus must be given to building caring communities.

Noddings (1992) argues that schools cannot achieve their academic goals for students without providing care and continuity for students. However, even if a caring school climate is not in place, there may be hope. McCombs and Whisler (1997) claim that caring classroom communities can ameliorate student feelings of frustration with, and alienation from, school.

\subsubsection{Caring classroom environments}

Many authors discuss the importance of teachers establishing and maintaining strong, safe, supportive, and caring learning communities (e.g., Brophy \& Good, 1974; Good \& Brophy, 2003; Haynes et al., 2003; McCabe, 1995). Alton-Lee (2003) also emphasises teachers must acknowledge the interdependence between the social and academic aspects of learning conditions and that caring and supportive environments are generated through teacher practices and quality teacher-student interactions. She strongly advocates the use of:

pedagogical practices [which] enable classes and other learning groupings to work as caring, inclusive, and cohesive learning communities. (pp. vi, 22)

Blum (1999) claims that caring is affected by one's temperament, interest, opportunity, and character. He sees the interpersonal bonds in a caring classroom as being deeper and stronger than those formed within a 'comfortable' classroom (e.g., Clark, 1997). He differentiates between care directed towards individuals and care directed at the collective or groups within the class. However, Noblit et al. 
(1995) believe that acts of individually directed care could have positive effects on the sense of care felt by the whole class.

In a United States based study of $6^{\text {th }}$ to $8^{\text {th }}$ grade students in two schools (one city and one suburban), Bosworth (1995) found that while most observed classroom interactions were pleasant and polite, the interactions did not involve care. Bosworth claims that few classroom opportunities exist to develop a caring community; hypothesising that in many classrooms the goal to improve academic performance leaves little time for personal interaction. Noddings (1992) advocates teachers and schools legitimising such time. Peterson (1992) also acknowledges and values teachers taking time to attend to students' experiences and feelings.

Haynes et al. (2003) provide a range of questions that they believe students consciously or subconsciously ask themselves in mathematics classrooms including:

If I reveal things about myself in this class, what will others think about me?

What does the teacher think of me when I make a mistake?

Does the teacher care about what I do or feel?

What do my classmates think of me when I ask for help?

When I work in small groups do my classmates want me in the group and want my ideas? (pp. 109, 110)

Considering questions such as these, a caring environment is clearly essential for students to be able to feel sufficiently comfortable in the classroom to be able to maximise their learning.

Peterson (1992) recommends using practices that help students develop a sense of community belonging, such as engendering a sense of shared endeavour and using learning contexts drawn from students' interests and experiences. Others emphasise the importance of establishing an orderly and safe environment (e.g., Bosworth, 1995; Haynes et al., 2003; Noblit et al., 1995). Clark (2001) discusses the relevance for Pasifika students of similar ideas and links teachers' high expectations for mathematics learners with respect for students' abilities and their comfort in the classroom.

Developing trust, advocated by Noddings (1992) and McCombs and Whisler (1997), also helps create a caring learning community. Lave and Wenger (1991) used a range of communities outside of schooling to develop their theories of community of practice and legitimate peripheral participation. They found these concepts to be useful tools 
for thinking about classroom learning environments. They describe learning as not merely situated in practice but as "an integral part of generative social practice in the lived-in world" (p. 35). They see communities as complex combinations of multiple factors and use the term 'peripherality' to describe the many and varied ways people can be involved within a community: more or less engaged and with differing feelings of belonging and inclusion. Lave and Wenger suggest that intrinsic personal rewards can come from being a member of a learning community and found that peripheral participation can lead to full community participation.

Peterson (1992) analyses the classroom in terms of community building practices, such as ceremony, ritual, rite, celebration, play, and critique. He argues these are essential elements of community life. He defines 'ceremony' to include opening and closing activities, gaining readiness for the day's learning, and acknowledging one another, stating these practices put shape and life into a learning community. He defines a class 'ritual' to be an activity that can signal a change in what is going to happen or what to do (e.g., writing the date, taking the role). A rite or rites are usually involved in the lesson opening ceremony and allow everyone to know what is happening. He continues that individual students may have their own personal rituals to help them get ready for doing their independent work (e.g., sharpening a pencil), which teachers should accommodate.

A model for fostering caring classroom communities, in which students are engaged both emotionally and intellectually, is proposed by Andersen (1995). He advocates using a seven-stage process: developing classroom caring; sharing and selfdisclosure; awareness; respect; faith; responsibility; and purposefulness, with each stage leading to the next.

Thus the literature provides common themes regarding the importance of establishing caring and effective learning environments conducive to learning and many ideas for classroom practices to help to create such environments.

\subsubsection{Behaviours of caring teachers}

There are many specific teacher practices that have been found to help develop caring mathematics learning communities, including:

- involving students in classroom decision-making (Alton-Lee, 2003; Haynes et al., 2003; Noddings, 1992; McCombs \& Whisler, 1997); 
- $\quad$ using 'safe' questioning practices (i.e., those that do not expose students to potential embarrassment or intimidation) (Bills, 2000; Clark, 2001; Noddings, 1992, 2003). For example, Noddings recommends against the use of 'coldcalling' (calling on students who have not offered a response);

- creating a sense of shared endeavour (McCombs \& Whisler, 1997; Peterson, 1992);

- encouraging and expecting respectfulness (Anthony \& Walshaw, 2007; Bosworth, 1995; Elias et al., 1997; Haynes et al., 2003; McCombs \& Whisler, 1997) and being respectful of students (Fraser, 2008); and

- incorporating pedagogies such as collaborative work (Anthony \& Walshaw, 2007; Haynes et al., 2003; Hill \& Hawk, 2000), stories and narratives (Noblit et al., 1995), and journaling (Ensign, 2003).

Noddings (1992, 1995) goes beyond advocating a caring teaching approach discussing teachers' responsibilities of teaching themes of care. Her approach includes intentionally teaching students how to care for others, themselves, plants and animals, and for their environment:

\footnotetext{
When we discuss teaching and teacher-learner relationships in depth, we will see that teachers not only have to create caring relations in which they are the carers, but that they also have a responsibility to help their students develop the capacity to care. (Noddings, 1992, p. 18)
}

Gay (2000) considers that teachers who really care for their students as people and for their performance honour their humanity, hold them in high esteem, have high academic expectations, and assist students to achieve these. She states that students respond to such practice by aiming high and working well. Gay considers teacher care to be evident in teachers' attitudes, expectations, and behaviours, and claims caring teaching to be one of the pillars of culturally responsive pedagogy (see later in this section).

Cowie and Bell (1999) found evidence in New Zealand science classrooms of 'carereferenced ways of acting' in how teachers acted on formative assessment information. They found that in explaining their practice, teachers often indicated that they would not want their classroom response to damage their relationship with their students, and reported that at times their actions were chosen in order to nurture these relationships. Models used to analyse data for their study, such as teacher "noticing", "recognising", and "responding" (pp. 108-112, 115) usefully inform this study, as they are involved with empathy and caring. For example, Cowie 
and Bell explain that 'responding' requires "reciprocity, empathy, spontaneity, and flexibility" (p. 111) and emphasise the importance of 'teacher wisdom' drawn from previous teaching experience to inform teaching decisions.

McCroskey (1992) identified three factors likely to lead students to perceive the teacher as caring about their welfare: empathy, understanding, and responsiveness. Teachers who are perceived by students as caring teachers use democratic interaction styles, acknowledge difference in developing behavioural expectations, model a 'caring attitude' to their own work, and provide constructive feedback (Wentzel, 1997).

There is some evidence of variation by ethnicity in the aspects of teacher-student relationships most valued by students (Thompson, 1998). Hudley and Daoud (2007), for example, found in their Californian study that teacher warmth was more highly valued by Latino/Latina students, and academic validation was more important to Anglo-American students, than by others.

The importance of using contexts relevant to students for engagement, motivation, and achievement is emphasised by many (e.g., Anthony \& Walshaw, 2007; Brophy \& Good, 1974; Eccles, 2004; Gay, 2000; Hill \& Hawk, 2000; Holloway, 2004; McCombs \& Whisler, 1997; Noddings, 1992; Stoll \& Fink, 1996). Haynes et al. (2003) found that such teaching motivated students because it encouraged social and emotional responses to lesson content, which in turn assisted with cognitive response. They found students felt heard and respected when their teachers solicited and used their everyday experiences, helping to develop a sense of trust in their teachers and to form affirming relationships. As a discipline in which much content is, or quickly becomes, abstract and is loosely or not directly related to students' lives, teachers of mathematics in particular are challenged to incorporate contexts that draw from the lives and interests of their students, particularly with contexts drawn from cultures other than their own. Use of real life contexts in mathematics is more likely than in comparatively context-rich subject areas (e.g., Social Sciences, Sciences) to require a deliberate teacher act. The use of contexts relevant to students' lives will be discussed further later in this section.

Teacher care is likely to be holistic in nature; however, for the purposes of exploring the detail of teacher care, three broad aspects of teacher care are now explored:

Care for students as individuals;

Care for students' mathematics learning; and

Care for students as culturally located individuals. 


\section{Care for students as individuals}

Teachers can show their care for students as individuals in a wide range of physical, verbal, aural, and empathetic ways. Teachers' body language is a strong clue to students that the teacher cares for them. Although all students are likely to hold differing preferences, a range of authors discuss the importance of affirming body language including smiling (Bosworth, 1995; Powell, 2000), facing students, touching (Bosworth, 1995; Noblit et al., 1995), showing they like their students (Hawk \& Hill, 2000), and sustained eye contact (Haynes et al., 2003; Noblit et al., 1995). Noblit et al. (1995) highlight that people's perceptions of touching are highly dependent on the individuals involved. Attitudes towards eye contact are known to vary across ethnicities and it is unlikely to be seen as a caring act by some, for example, some Māori $(\mathrm{H}$. Easton, personal communication, 19 April, 2006), some Pasifika (F. Rimoni, personal communication, 28 June, 2006), and Navajo (Greenbaum \& Greenbaum, 1983).

Bosworth (1995) interviewed over 100 students regarding what they expected from caring teachers. The students' views included that caring teachers would be polite, use praise, see students as individuals, talk with and listen to them, and give of their time and of themselves. Other authors concur with many of these ideas. Being polite is achieved in a variety of ways. Peterson (1992) and Noblit et al. (1995) discuss the importance of acknowledging students, in particular by ensuring they greet and farewell them and by talking with them. Students in the Noblit et al. study (1995) stated that a teacher talking with them was a sign of teacher care. The researchers saw such discussions as fundamentally important for implementing teacher care, as through talk students reveal their lives and thoughts, thus providing teachers with a ready means of supporting learning. Taking time to show interest in students as people, and teachers being and sharing themselves with students have also been highlighted as effective teacher practice by others (e.g., McCabe, 1995; Powell, 2000).

Hawk and Hill (2000) advocate using students' names and Noddings (2003) encourages teachers to admit and apologise when appropriate and to explain their practice, allowing students to understand why they are making specific teaching decisions. Such practices are likely to help students feel cared for, that they are an integral part of the lesson, and develop a sense of shared endeavour. By explaining their practice, teachers are also modelling that explanations are a useful tool for understanding others. This is consistent with Elias et al.'s (1997) discussion suggesting teachers should aim to understand rather than to judge, McCombs and Whisler's (1997) emphasis on developing sharing class communities, and Bills' 
(2000) emphasis on inclusive classroom language (e.g., using 'we' rather than 'you' when discussing classroom activities).

Use of specific praise is advocated in part to help encourage students' selfconfidence (Good \& Brophy, 2003; Hawk \& Hill, 2000). Many authors (e.g., Elias et al., 1997; Hawk \& Hill, 2000; Powell, 2000) encourage teachers to give of themselves, claiming that sharing parts of their personal lives and encouraging this of their students builds empathy, allows recognition of shared experience, and strengthens a sense of community. The importance of teachers knowing their students as individuals and the ability to relate to students on a personal level as well as a teacher level is commonly emphasised (Bosworth, 1995; Brophy \& Good, 1974; Gay, 2000; Hill \& Hawk, 2000; Haynes et al., 2003; McCombs \& Whisler, 1997; Noddings, 1992, 2003). Noddings (1992) believes that knowing each other creates a sound foundation for caring interactions.

Hawk and Hill (2000) and Mead (2003) emphasise the importance of care for students' self-esteem (mana) suggesting teachers working one-to-one speak quietly when appropriate. Friedel, Marachi, and Midgley (2002) go further to link teachers' care not to embarrass students to increased student risk taking and reduced maladaptive behaviour.

Appropriately used, humour is a useful tool for developing rapport (Hawk \& Hill, 2000; McCabe, 1995; Powell, 2000). It is possible that this element is even more pertinent to New Zealand students (in particular, Māori and Pasifika students) than those from other countries, as humour is a common and powerful strategy used in these cultures for interpersonal relations.

Many authors (e.g., Alton-Lee, 2003; Bills, 2000; Noddings, 2003; Peterson, 1992) encourage teachers to consider carefully the way they exercise authority. Downgrading the power relationship and using classroom guidelines to facilitate learning rather than to emphasise compliant behaviour or control are seen as strategies conducive to developing caring relationships.

There is general agreement that a caring teacher shows empathy (Bosworth, 1995; Elias et al., 1997; Hill \& Hawk, 2000; Peterson, 1992), responds to needs (Elias et al., 1997; McCombs \& Whisler, 1997; Noddings, 2003), and is non-confrontational (Hill \& Hawk, 2000). They are supportive (Haynes et al., 2003) and show they listen to students (Bosworth, 1995; Elias et al., 1997; Hill \& Hawk, 2000; Haynes et al., 2003; Stoll \& Fink, 1996). 


\section{Care for students' mathematics learning}

Caring teachers expect all students to do well; they do what it takes to the best of their abilities to help each pupil achieve. (Stoll \& Fink, 1996, p. 192)

This section presents ideas from a range of literature regarding how teachers can show they care for students' mathematical progress. This will be discussed within two areas, care for learning in general and care for mathematics learning.

There is a range of ways in which teachers can show care by encouraging students, for example, to work hard, do well, maintain focus on and take pride in their work, and answer questions (Brophy \& Good, 1974; Hill \& Hawk, 2000; Haynes et al., 2003; Noblit et al., 1995; Powell, 2000). An encouraging teacher also acknowledges mathematical success and effort (McCombs \& Whisler, 1997) and enables affirmation by students' peers (Brophy \& Good, 1974; Hill \& Hawk, 2000; McChesney, 2009). All aspects relating to teacher encouragement are also consistent with Alton-Lee's (2003) view that effective "teachers manage the evaluative climate so that academic norms are not undermined but supported by social norms" (p. x).

Hackenberg (2005) offers a model that links mathematics learning and caring classroom relations. She describes personal interactions as 'depleting' (leaving a feeling of being drained or diminished) or 'stimulating' (leaving a feeling of being boosted) and contends that both types of interactions are necessary for learning in any area. In mathematics learning, there is a particularly high potential for feelings of depletion because of factors including: the differences between mathematical thinking and thinking in other curriculum areas (Watson, 2002); the difficulty of the subject; some students' negative attitudes to learning mathematics; and the dependence for new learning of prior mathematics learning.

Hackenberg (2005) believes that prolonged periods of depletion can lead to students feeling uncared for by their teachers. She sees teacher care for mathematics learning as working towards a balance of depletion and stimulation effects and engendering confidence in students that they can overcome the depletion feelings they may encounter. For these to be achieved teachers must be in tune with students' mathematical thinking and spend time on mathematical confidence and pleasure building activities.

The students in Bosworth's (1995) study also found encouragement, assistance, and being part of a positive learning community important for their learning. They stated that caring teachers showed tolerance, liked helping students and meeting 
individual needs, were success orientated, provided a structured classroom environment, checked for understanding, planned fun activities, and gave praise and encouragement.

Many authors agree that caring teachers help when needed (e.g., Brophy \& Good, 1974; Elias et al., 1997; Gay, 2000; Hill \& Hawk, 2000; Powell, 2000). The students in Bosworth's (1995) study did not relate teacher care directly to curriculum content but it can be difficult for students to notice the caring teacher practices most closely related to curriculum delivery. For example, Noddings (1992) suggests that reminding students early in a lesson of prior learning specifically useful for the lesson content is an effective and caring teaching strategy. Students may or may not identify such practice as a deliberate teacher act of care for learning.

\section{Caring for students as culturally located individuals}

Caring-centred approaches foregrounding teacher-student relationships and addressing "students' needs with explicit attention to students' cultural contexts" (Pang, 2005, p. 389) are also advocated within the literature regarding teaching in multicultural classrooms. Alton-Lee (2003) addresses diversity in a range of ways. She advocates creating effective links "between school and other cultural contexts in which students are socialised, to facilitate learning" ( $p$. vii), as the third of ten research-based characteristics of quality teaching. The descriptors for this characteristic include:

Quality teaching respects and affirms cultural identity (including gender identity) and optimises educational opportunities. (Alton-Lee, 2003, p. vii)

Descriptors of other quality teaching characteristics (Alton-Lee, 2003) also pertain to addressing diversity, for example:

Pedagogical practices pro-actively value and address diversity.

The language and practices of the classroom are inclusive of all students.

Teachers use class sessions to value diversity, and to build community and cohesion. (Alton-Lee, 2003, p. vi)

Gay (2000) insists that many types of ability are lying untapped in ethnically diverse students and advocates culturally responsive teaching as "a means for unleashing the higher learning potentials of ethnically diverse students by simultaneously cultivating their academic and psychosocial abilities" (p. 20). Wlodkowski and Ginsberg (1995) offer a framework for teaching and learning which is sensitive to cultural differences. The framework includes four interactive elements: establishing 
inclusion, developing attitude, enhancing meaning, and engendering competence. They claim a link between motivation and culture, and discuss the relationships between emotion, engagement, and intrinsic motivation. They also put forward factors they believe can inhibit effective culturally responsive practice (e.g., teachers blaming the situation and the students for lack of student achievement, and assessment requirements and modes).

Wlodkowski and Ginsberg (1995) state "to be effective in multicultural classrooms, teachers must relate teaching content to the cultural backgrounds of their students" (p. 17). Many other authors (e.g., Elias et al., 1997; Hill \& Hawk, 2000; Haynes et al., 2003; Noddings, 1992; Peterson, 1992) agree with their view of the importance of drawing learning contexts from the students' culturally located expertise, knowledge, interests, and experiences, and of providing learning experiences which "nurture and serve the community" (Elias et al., 1997, p. 45). Gay (2000) presents evidence from a range of research projects which supports these views, including one project (McCarty, Lynch, Wallace, \& Benally, 1991) that found that when the students' "social experiences were incorporated into curriculum and instruction, and their cultural and linguistic resources were used to solve academic problems the Navajo students became physically energized, intellectually engaged, and verbally fluent in the classroom" (Gay, 2000, p. 14).

Ways of responding to students' cultural identities and communities within learning programmes is a matter of increasing interest amongst educational researchers. Howard, Perry, and Butcher (2006) describe key features of the processes used to build the involvement of parents and communities with schools at two project sites towards collaborative development and implementation of mathematics teaching strategies (e.g., Perry \& Howard, 2008) to enhance the learning outcomes for Australian Aboriginal students. Other strategies and investigations aimed at improving links between communities and mathematics learning include the Algebra Project (Moses \& Cobb, 2001), teaching mathematics for social justice (e.g., Gutstein, 2003), building awareness of cultural diversity issues pertinent to education (e.g., Philpott, Nesbit, Cahill, \& Jeffery, 2004), and working within communities to develop mathematics learning experiences and processes relevant to students (e.g., Nicol et al, 2007).

McCombs and Whisler (1997) advocate teachers expanding rather than diminishing individual difference in their classrooms, and recommend incorporating aspects of the diversity within their class into class time. Hawk and Hill (2000) encourage 
teachers to relate to culturally diverse students on a personal as well as a teacher level, to understand and allow for students' code/s of behaviour and cultural responsibilities, and to acknowledge their cultural knowledge and expertise. Successful teachers of minority students consider students' cultures, communities, and languages as assets and recognise that all cultural groups have contributed to mathematical knowledge (Holloway, 2004). Noddings (2003) goes further still advocating that teachers know students well enough that classroom interactions and relationships can be home-like and that schools and classrooms provide family-type relationships like students experience at home.

Thus the literature informs us that in order to show caring for students' cultural identities, teachers must recognise, understand, and value the individuality and diversity of the students in their classes, and must link mathematics learning to the cultural identities of their students.

\subsection{Discussion}

There is ample evidence that caring teacher-student relationships are vital for effective learning and the literature offers much to help describe caring teacher practices and informs this study. The consistency across studies and authors and across student groups of differing contexts and ethnicities suggest that the most salient aspects of teacher care may be stable across time and place. What is missing, however, are studies which describe teacher care as experienced and preferred by New Zealand students, in particular our Māori, Pasifika, and low socioeconomic students.

Furthermore, much of the literature is concerned with classrooms in general and does not focus specifically on mathematics classrooms. Mathematics is both a key area for the New Zealand Ministry of Education's strategic national Literacy and Numeracy initiatives and an important subject for students in helping to keep future study and career options wide. It is very important, therefore, to find out how mathematics teachers can enhance students' opportunities in mathematics by describing what assists with establishing and maintaining effective caring teacherstudent relationships. 


\section{CHAPTER THREE}

\section{Methodology and Implementation}

This chapter describes and justifies the theoretical methodological perspectives which led to the study design (Section 3.1), the development process used for creating the data gathering tools (Section 3.2), the study participants (Section 3.3.), data collection and feedback to participants (Section 3.4), and data analysis and description (Section 3.5).

\subsection{Study design}

A sociocultural epistemological stance was selected for the study. Sociocultural research privileges the idea that learning and understanding "originate on the social plane, in social interactions, in language, and/or in enculturation" (Lerman, 2006, p. 356) and provides a suitable framework for studies exploring issues of equity. The following considerations also informed the choice of epistemological stance for the study. The research questions are centred within the context of multicultural Year 10 classrooms on investigating and understanding teacher beliefs and behaviours, and how teacher behaviours are received and interpreted by their students. The study was intended to generate a "theory or pattern of meaning" (Creswell, 2003, p. 9). To thoroughly examine and understand the complexity of participant views, and best answer the research questions (Pirie, 1998), it is important to spend considerable time within the context of the investigation in order to gather and draw meaning from the first-hand perspectives of multiple participants (including those of the researcher). It is recognised that the researcher's background, beliefs, and experiences affect researcher decision-making regarding interpreting the context and data. Such effects and ways to reduce these should be considered particularly when the researcher and the researched are of different cultural backgrounds. A naturalistic (Merriam, 2001; Patton, 2002) stance was taken in order to minimise the effects of the research process on the data.

Three alternative knowledge claims (postpositivism, pragmatism, and an advocacy/participatory paradigm) (Creswell, 2003) were rejected for the study (Table 3.1) because of the lack of pre-existing theory, the likely strong relationship between the data and contextual factors, and the investigative nature of the study. 
Table 3.1: Reasons for rejection of potential paradigms

\begin{tabular}{|l|l|}
\hline $\begin{array}{c}\text { Knowledge Claim/ } \\
\text { Worldview (Creswell, } \\
\text { 2003) }\end{array}$ & \multicolumn{1}{c|}{$\begin{array}{c}\text { Main reason/s the knowledge claim/worldview } \\
\text { was rejected for this study }\end{array}$} \\
\hline Postpositivism & $\begin{array}{l}\text { There is no existing theory for the specific context of the study, } \\
\text { therefore the focus was not to test and refine theory. } \\
\text { The research aim was to investigate and understand rather than } \\
\text { to find causes or outcomes. } \\
\text { The research focus was not well suited to an empirical } \\
\text { investigation because the data required were likely to be highly } \\
\text { influenced by the specific context within which it was gathered. }\end{array}$ \\
\hline Pragmatism & $\begin{array}{l}\text { The research did not start with a problem to solve, to analyse } \\
\text { consequences of actions, or to determine a real-world practice- } \\
\text { based solution. }\end{array}$ \\
\hline Advocacy/participatory & $\begin{array}{l}\text { The research aim was not to intertwine the study with a political } \\
\text { agenda, to set an agenda for reform, or to collaborate with or } \\
\text { empower research participants. } \\
\text { Although equity issues are important drivers for the research, the } \\
\text { study did not start from the stance of an issue or problem in } \\
\text { society. }\end{array}$ \\
\hline
\end{tabular}

The main focus of the study was to interpret teacher-student classroom interactions in terms of teacher care. Qualitative methods were used to provide the bulk of the study data as they can lead to clear descriptions of beliefs and behaviours, and as such, are best suited to a sociocultural theoretical frame. An ethnographic strategy of inquiry (Creswell, 2003; Grbich, 2007; Merriam, 2001) was selected as the primary methodology for the study as it provides the best methodological fit with sociocultural perspectives (Pirie, 1998) and the research questions. Ethnographic studies allow for flexibility in the research process, an essential factor for classroom contexts in order to accommodate school and teacher needs and constraints. Furthermore, ethnographic studies involve intense focus on intact context-based groups within their natural setting over extended periods (Creswell, 2003). They draw from participants' own voices to help interpret their 'lived experiences'. These were highly appropriate strategies for understanding and describing the development and maintenance of teacher-student relationships over time.

Narratives and phenomenology were considered for the study to enable illumination of the "essence" (Creswell, 2003, p. 15) of teacher care. However, these methodologies were rejected in order to allow understanding of the phenomenon of teacher care across a range of settings as actioned and experienced by many teachers and students, rather than to focus in depth on teacher care within the broader context of the lived experiences of a relatively small number of participants. The use of case study as a methodology was also considered, as one which would allow comparisons of holistic pictures of teacher care across the study classes. 
However, this method was rejected because ethnographic research held greater opportunity for generation of theory and description of patterns across different teacher, school, and classroom contexts.

Grounded theory involves multiple stages of data collection carried out concurrently with analysis throughout a study allowing for "theoretical sampling" (Charmaz, 2006, p. 96; Denscombe, 2001, p. 16; Pirie, 1998). This process allows continual comparison of new data with emerging theoretical categories (Creswell, 2003; Merriam, 2001). A benefit of grounded theory is that information gathered from all sources (e.g., from literature, participants, researcher journals, emails...) can be included as data that help to inform the evolving theory. Grounded theory was considered for the study but as the study was to encompass two years of data collection with different participants in each of the years, grounded theory was rejected as the sole methodology in order to allow common questionnaire data to be gathered for all study groups. However, elements consistent with grounded theory were used in order to maximise responsiveness to new information and interim and informally developed theory. For example early study data were used to inform interview questions in later data collection.

Qualitative methods can provide rich, thick descriptions (Creswell, 2003; Merriam, 2001; Patton, 2002) of the phenomenon under investigation but are less suitable for generalising to populations or comparing results from groups within the study participants. Non-experimental quantitative data (Creswell, 2003) allow closer examination of the participants' thoughts, feelings, and motivations, identification of patterns and trends, and comparison of data from different groups. Quantitative methods were also useful for the study in order to enable further understanding of the phenomena of teacher care, to compare data from different subgroups, and to assist in generalising findings (Creswell, 2003).

A typology of research purposes and links between research purpose/s and suitable methodologies (Newman, Ridenour, Newman, \& DeMarco, 2003) was investigated in order to confirm the appropriateness of combining qualitative and quantitative methodologies for this study. The research purposes for this study best matched the second and fifth of nine general purposes for social science research ("add to the knowledge base" and "understand complex phenomena") (p. 175). Traditional paradigms for these purpose groups are quantitative and qualitative respectively (Newman et al., 2003) but both purposes provide opportunities for mixed methods research, with qualitative methods aiding in developing theory and quantitative 
methods allowing greater responsiveness to multiple stakeholders, confirming the appropriateness of using a mixed methods approach for this study.

Informally researchers have been combining qualitative and quantitative methods throughout the twentieth century; however, more formal descriptions, designs, and processes of mixed methods research (i.e., using a mix of quantitative and qualitative methodologies and methods) have a comparatively recent history, having evolved from ideas of triangulating information from different sources (Campbell \& Fiske, 1959). Mixed methods research, still emerging and developing as a separate methodological movement (Tashakkori \& Teddlie, 2003), seeks to use triangulation of quantitative and qualitative approaches (Jick, 1979; Patton, 2002) to allow convergence of study findings across approaches and to help overcome the limitations and biases of each method. Mixed methods research can provide answers to questions that cannot be answered by qualitative or quantitative approaches alone. It allows for an expanded understanding of the data through combining research methods and tools from both paradigms (e.g., observations, interviews, and surveys). It enables results from one approach to inform the other or inform specific aspects of a study, and can help enhance the transformative nature of the findings by allowing focus on results from subgroups (Creswell \& Plano Clark, 2007; Tashakkori \& Teddlie, 2003). Further strengths of mixed methods research include:

- meaning can be enhanced through the mutual support of words, descriptions, diagrams, and numerical values;

- concerns of diverse groups can be appropriately addressed; and

- insights may be gained that would have been missed if only a single paradigm was used (Johnson \& Onwuegbuzie, 2004; Mertens, 2003).

Although varied views existed in the recent past regarding the suitability of mixing quantitative and qualitative approaches (Boulton \& Hammersley, 2006; Johnson \& Onwuegbuzie, 2004), mixed methods research is now well established and more and more frequently used for research across a wide range of disciplines (Alexander, 2008; Creswell, 2003; Johnson \& Onwuegbuzie, 2004; Tashakkori \& Teddlie, 2003). The challenges posed by mixed methods research include the need for extensive data collection, time-intensive analysis of text-based and numeric data, and researcher familiarity with both qualitative and quantitative paradigms and techniques (Creswell, 2003; Tashakkori \& Teddlie, 2003). 
A fundamental principle of mixed methods research design is to combine methods in such a way that complementary strengths are utilised and overlapping weaknesses avoided (Johnson \& Turner, 2003). Consideration was given to this principle at two stages of the study, in particular, during the research design (Section 3.1) and the development of the data gathering tools (Section 3.2).

In summary, the study draws from a constructivist framework and primarily takes an ethnographical stance. Within a mixed methods approach, the majority of study data were qualitative in nature supported by some quantitative data.

\subsubsection{Data collection methods}

Classroom environments are exceedingly complex. They are places where many individuals from a variety of backgrounds, experiences, and situations, and with a variety of personalities, priorities, belief systems, interests, and biases meet for learning within the wider school and other social contexts. Classroom actions and interactions include multiple inter-related and independent events occurring simultaneously and quickly. What happens within a classroom is affected by factors internal to the lesson itself as well as external factors such as links with the previous lesson, an impending event, and how the teacher interacts with students outside the lesson times.

Data collection methods and tools must suit the purpose of the study (Denscombe, 2001; Pirie, 1998; Wragg, 1999). The study design included predominantly qualitative methods supported by quantitative data collection (Table 3.2). The central data collection method for this study was chosen to be classroom observation as it would enable the words, actions, and reactions (Good \& Brophy, 2003) of the 'carer' and the 'cared for' to be observed as they happened within the context of the classroom. Observation also allows aspects of body language (identified as a vital area to explore by both the Māori and Pasifika cultural advisors) to be recorded and incorporated within this study.

Questionnaires, interviews, and audio-recording of observed lessons were used to supplement observational data to allow participants' perceptions to be gathered and crosschecked with observational data, consistencies between teacher and student behaviours and their thoughts and feelings to be explored, researcher biases to be ameliorated, and to enhance the quantity and mix of qualitative and quantitative data, thus creating a more comprehensive data set. During the analysis of study data it became apparent that some teacher-student interactions were out of range of the audio-recorder and hence data which may have shown examples pertinent to 
the study may have been missed. Due to the primary emphasis on observational data, the quantity of data already being collected, and the triangulation methods used to ensure validity, it was decided not to include a further data collection method to capture these conversations.

Using a range of data collection methods is important given that each method (observations, questionnaires, and interviews) has different practical constraints, strengths, and weaknesses in relation to examining the research questions. Using concurrent triangulation (Creswell, 2003), qualitative and quantitative methods were integrated within data gathering instruments (observation schedules and questionnaires) and data of both types used in parallel and together to inform analysis and reporting.

\section{Table 3.2: Data gathering methods}

\begin{tabular}{|c|c|c|}
\hline $\begin{array}{l}\text { Data gathering } \\
\text { method and } \\
\text { participants }\end{array}$ & $\begin{array}{l}\text { Purpose/s (within the contexts of students as } \\
\text { individuals, students as mathematics learners, and } \\
\text { students as culturally located individuals) }\end{array}$ & $\begin{array}{l}\text { Qualitative or } \\
\text { Quantitative }\end{array}$ \\
\hline $\begin{array}{l}\text { Classroom } \\
\text { Observation }\end{array}$ & $\begin{array}{l}\text { To collect: } \\
\text { - ways in which teachers demonstrate care during } \\
\text { mathematics lessons; } \\
\text { - how acts of teacher care are received by students; } \\
\text { and } \\
\text { - student-initiated interactions with the teacher (as } \\
\text { indicators of the strength of relationships and the } \\
\text { level of engagement with learning). }\end{array}$ & $\begin{array}{l}\text { Predominantly } \\
\text { qualitative }\end{array}$ \\
\hline $\begin{array}{l}\text { Audio-recording } \\
\text { of observed } \\
\text { lessons }\end{array}$ & $\begin{array}{l}\text { To collect real-time verbal teacher-whole class } \\
\text { interactions and teacher-student interactions. }\end{array}$ & Qualitative \\
\hline $\begin{array}{l}\text { Questionnaires } \\
\text { (teachers) }\end{array}$ & $\begin{array}{l}\text { To collect teachers' perceptions of: } \\
\text { - mathematics learning; } \\
\text { - effective ways to demonstrate teacher care; } \\
\text { - effective ways to establish and maintain rapport; } \\
\text { and } \\
\text { - } \quad \text { students' views of teacher practice. }\end{array}$ & Both \\
\hline $\begin{array}{l}\text { Questionnaires } \\
\text { (students) }\end{array}$ & $\begin{array}{l}\text { To collect students' perceptions of: } \\
\text { - mathematics learning; } \\
\text { - effective ways teachers use to demonstrate care; } \\
\text { - effective ways teachers use to establish and } \\
\text { maintain rapport; and } \\
\text { - } \quad \text { students' views of teacher practice. }\end{array}$ & Both \\
\hline $\begin{array}{l}\text { Semi-structured } \\
\text { interviews } \\
\text { (teachers) }\end{array}$ & $\begin{array}{l}\text { To collect teachers' perceptions of: } \\
\text { - how they get to know students and how well they } \\
\text { know their students; } \\
\text { - how they establish and maintain rapport with } \\
\text { individuals and the class as a whole; } \\
\text { - the challenges to establishing and maintaining } \\
\text { rapport with individuals and the class as a whole; } \\
\text { and } \\
\text { - the importance of teacher care for their students' } \\
\text { learning. }\end{array}$ & Qualitative \\
\hline
\end{tabular}




\begin{tabular}{|l|l|l|}
\hline $\begin{array}{c}\text { Data gathering } \\
\text { method and } \\
\text { participants }\end{array}$ & $\begin{array}{c}\text { Purpose/s (within the contexts of students as } \\
\text { individuals, students as mathematics learners, and } \\
\text { students as culturally located individuals) }\end{array}$ & $\begin{array}{l}\text { Qualitative or } \\
\text { Quantitative }\end{array}$ \\
\hline $\begin{array}{l}\text { Semi-structured } \\
\text { interviews } \\
\text { (students) }\end{array}$ & $\begin{array}{l}\text { To collect students' perceptions of: } \\
\text { how well their teacher knows them and how they } \\
\text { would prefer their teacher to get to know them; } \\
\text { how their teacher establishes and maintains } \\
\text { rapport with the class and with them individually; } \\
\text { and } \\
\text { the importance of teacher care for their learning. }\end{array}$ & Qualitative \\
& & \\
\hline
\end{tabular}

Triangulation enhances the validity of the data set by ensuring the use of many people's perceptions to inform the researcher's interpretations of observed events. The chosen range of data collection methods allows for methodological triangulation (more than one data gathering method including both qualitative and quantitative methods) and data triangulation (data collected from more than one source and at different times) (Denzin, 1985). The study overview shows the intended purposes for, and relationships between, the data collection methods (Table 3.2, Figure 3.1).

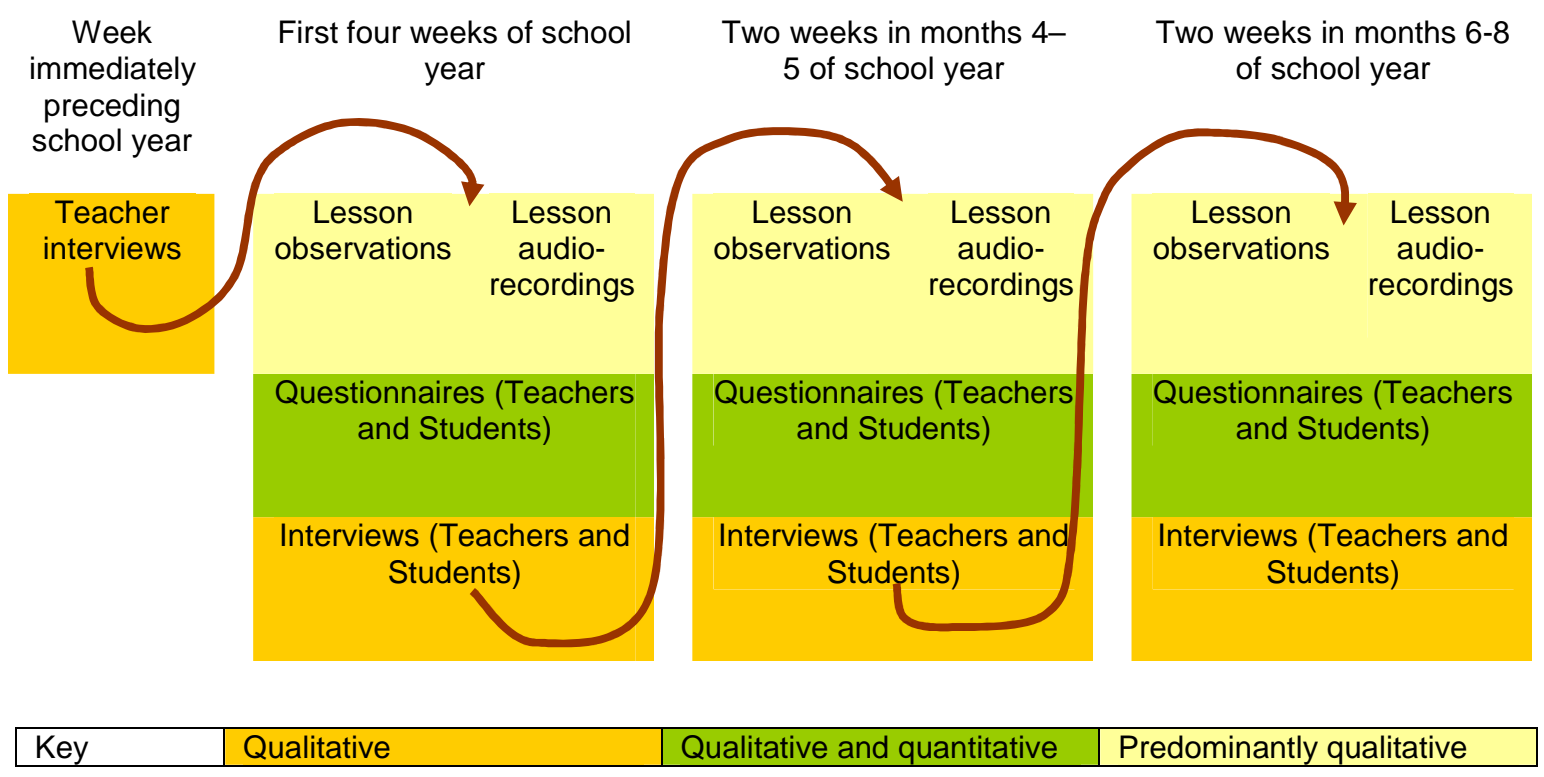

Figure 3.1: Study design

To gather baseline data on teachers' views regarding teacher-student relationships and establishing rapport with their class, an initial interview was held with each teacher in the week immediately prior to the teacher meeting the study class. Three rounds of data gathering were completed with each teacher and their class, with classroom observation, then questionnaires, and lastly interviews in each set (Figure 3.1). The first data gathering round was held in the first weeks of the school year in order to capture how teacher-student relationships were developed during this time. The second and third rounds were intended for data collection once the teacherstudent relationships were in place in order that their characteristics and ways in 
which the relationships were maintained could be recorded within two time periods spread through the school year.

The sequence of the data gathering methods was designed to enable each set of classroom observations and questionnaire results to inform the subsequent teacher and student interviews. Furthermore, carrying out the lesson observations before gathering the questionnaire and interview data in each round would provide a body of shared experience between researcher and participants, enhancing teachers' and students' comfort in providing questionnaire and interview data, and the researcher's understanding and interpretation of interview responses. The order also allowed examination of specific observed phenomenon and questionnaire responses in the interviews.

Theoretical sampling, often used within grounded theory (Creswell, 2003; Denscombe, 2001; Pirie, 1998), is an iterative process in which each stage of theory development informs the next set of data collection. Theoretical sampling was used in this study in order that all useful information from the literature, conversations with cultural advisors and others, as well as that gained through interim findings using data from all gathering methods could be used in a responsive and efficient way to inform further data collection. Examples illustrating how the study design allowed interim findings, further reading, and consultative discussions to be used to enhance data collection include:

- data gathering being carried out over two years (Phase One in the first year and Phase Two in the second) allowing later data collection to be informed by discussion of earlier data and researcher reflection;

- the inclusion of further interview questions as the study progressed to probe areas of interest; and

- modifying Questionnaires Two and Three prior to their initial use to capture further detail in the data.

To further increase data validity, this study included repeated observations at several research sites, "peer examination" (Merriam, 1998, p. 204), and "member checking" (Kyriacou, 1990, as cited by Sanger, 1996, p. 40) of selected data summaries. 
The study's predominant data gathering methods (observation, questionnaires, and interviews) are now discussed in turn to describe their methodological strengths and weaknesses, further rationale for their inclusion, and the way in which they were used.

Systematic focused observation allows efficient, reliable data collection within the natural setting of the phenomenon being explored (Denscombe, 2001; Johnson \& Turner, 2003) and is an important tool for understanding classroom settings:

Good classroom observation can lie at the heart of both understanding professional practice and improving its quality. (Wragg, 1999, p. 17)

Strengths of observational data collection include that it allows relatively objective measurements of behaviour, access to the contextual factors of natural social settings, and provides useful data for describing phenomena and contexts (Johnson \& Turner, 2003).

There are many quantitative and qualitative methods for recording classroom observational data (Wragg, 1999). Using a combination of observation methods allows a breadth of data types to be collected and can help reduce subjectivity. In this study a combination of counts, ratings, category recording, and annotations were used (Appendix 3). Neither 'event' nor 'time' sampling methods (Good \& Brophy, 2003) were used in their pure forms but adaptations of these were used to record comparative frequencies of events alongside specific quotes or examples particularly pertinent to the research questions. Sufficient observations were completed in each data round for distinct patterns of teacher and student behaviours to be apparent, for effects of researcher presence to be minimised, and to ensure pertinent examples of teacher practice could be recorded.

Weaknesses in observational data gathering include that the reasons underlying behaviours can be unclear, interpretive validity can be low (data are susceptible to observer bias), and data gathering and data analysis can be time-consuming (Johnson \& Turner, 2003). The accuracy of observation data can be limited by the complexity of the classroom (how much is happening at once), misinterpretation of events, oversimplification of behaviours (Denscombe, 2001), the observer's background, past experiences, and prejudices (which can cause selective noticing and hinder objectivity), trying to interpret information prematurely, and changes in classroom behaviour due to the observer's presence (Angrosino \& Mays de Perez, 2000; Good \& Brophy, 2003; Wragg, 1999). Using observation in combination with 
other data collection methods and being aware of researcher preconceptions that may reduce objectivity help to ameliorate the impact of these factors.

Questionnaires are an important component of mixed methods research as data gathered by way of questionnaire can be used to inform the development of subsequent data collection tools (Johnson \& Turner, 2003). They enable a large amount of quantitative and qualitative information to be gathered efficiently (Denscombe, 2001, p. 27) and allow participants time to give considered replies (Wragg, 1999). Questionnaires are useful for measuring attitudes, convenient for analysis of closed-ended items, and can have higher perceived anonymity than interviews or observational data gathering methods (Johnson \& Turner, 2003). However, good questionnaires can be difficult to construct (Good \& Brophy, 2003) and responses are generally less detailed than those collected through interviewing. As with interview data, the accuracy of data from questionnaires is susceptible to respondents' honesty, perceptions, and emotions. In spite of these issues, the large quantity of data produced by questionnaires can be used to sensitise the researcher to key trends and salient issues, thus maximising the effectiveness of subsequent interviews (Merriam, 1998).

Care was taken to maximise the validity of the data in constructing the study questionnaires. Principles for questionnaire design were used to guide development; for example, ensuring the questions matched the research questions, using simple, clear, precise items, measuring abstract aspects using multiple items, and piloting (Johnson \& Turner, 2003). These principles were used alongside considerations and information regarding questionnaire development (S. Fontaine, personal communication, 16 May, 2005) to create draft questionnaires.

The choice of focused observation necessitated the use of interviewing to help understand and describe what had been observed (Angrosino \& Mays de Perez, 2000). Interviews generate detailed data, provide insights into participants' thoughts and feelings, and allow data to be checked as they are gathered. Interviews are also useful for assessing attitudes (Johnson \& Turner, 2003). Semi-structured interviewing (Denscombe, 2001; Merriam, 1998) was selected for this study as it allowed for a conversational style, consistent issues to be addressed by all participants, and the flexibility to pursue in depth particular areas of interest arising from responses. 
Interviews are time consuming and therefore not all participants' views can be sought. Furthermore, consistency and objectivity can be hard to achieve in interviews. The perceived anonymity of respondents is low, data analysis can be time-consuming, and interview data are susceptible to 'interviewer effect', i.e., responses may be influenced by participant biases, aspirations, or by what they believe the interviewer may wish to hear (Denscombe, 2001; Johnson \& Turner, 2003). Furthermore, teachers may be not fully aware of their own classroom practice (Good \& Brophy, 2003) and may therefore have difficulty in answering questions designed to help the researcher understand their motivations and thoughts about their practice. Attempts to minimise these effects are discussed in Section 3.4.

All interviews were recorded and transcribed and researcher reflections noted following each set of interviews.

\subsubsection{Researcher-participant relationships}

Situating the researcher on the periphery of the classroom community as 'observeras-participant' (Angrosino \& Mays de Perez, 2000) is common in classroom research and was the positioning chosen for this study. This role was chosen in order to maximise the quality of the data by achieving a contextually appropriate balance between the purely objective and distant observer and one fully immersed and involved. Researcher/practitioner links are important to ensure educational studies are relevant and grounded in everyday practice (Pring, 2000) and many encourage connection between researcher and participants (Fontana \& Frey, 2000; Mertens, 2005), particularly in research involving Māori (Bishop \& Glynn, 1999; Smith, 2005) and Pasifika participants (Anae, Coxon, Mara, Wendt-Samu, \& Finau, 2001) such as this study.

Establishing and maintaining rapport with participants is vital for effective research involving Māori and marginalised communities:

For indigenous and other marginalized communities, research ethics is at a very basic level about establishing, maintaining, and nurturing reciprocal and respectful relationships, not just among people as individuals but also with people as individuals, collectives, and as members of communities, and with humans who live in and with other entities in the environment. The abilities to enter pre-existing relationships; to build, maintain, and nurture relationships; and to strengthen connectivity are important research skills in the indigenous area. They require critical sensitivity and reciprocity of spirit by a researcher. (Smith, 2005, p. 97) 
Furthermore, this practice is consistent with kaupapa Māori research (Bishop, 2005), Māori-centred research (Cunningham \& Durie, 1998), and with suitable approaches for researcher conduct (Smith, 2005, p. 98).

The researcher's intended relationship with teacher and student participants was as a respectful, non-judgmental observer, conveyed by being unobtrusive and uninvolved in the mathematics teaching and learning yet being pleasant and responsive to greetings and questions about the study. These compromises with a purely objective researcher approach, in which even eye contact with students is avoided (Good \& Brophy, 2003), were made to be consistent with the intended researcher-participant connections and enhance students' trust and comfort levels with the researcher in order to minimise the effect of the researcher on observational data and facilitate quality in questionnaire and interview responses.

\subsection{Developing culturally responsive data gathering tools}

A rigorous process was used to develop the data gathering tools in order to ensure they were fit for the purpose. This section describes the rationale and processes used to develop the data gathering tools for this study (Sections 3.2.1 and 3.2.2) with particular focus on describing ways in which cultural responsiveness was ensured. The importance of consultation with cultural advisors, student advisors, teacher experts, trial classes and teachers, alongside the literature, to the development of the data gathering tools is described and illustrated with examples.

Data gathering in multicultural classrooms is particularly complex as teachers, students, and classroom observers often hold differing worldviews, values, perspectives, behaviours, and interpretations of verbal and non-verbal communication. The challenge faced by qualitative researchers in recording the views and actions of participants from cultures other than their own is considerable:

\footnotetext{
Any gaze is always filtered through the lenses of language, gender, social class, race, and ethnicity. There are no objective observations, only observations socially situated in the worlds of - and between - the observer and the observed. (Denzin \& Lincoln, 2000, p. 19)
}

All researchers have a range of levels of immersion in their own and society's dominant and less prominent cultures (Banks, 2006). Challenges to effective data collection often exist in cross-cultural classroom-based research, for example, despite employing 'auto-ethnographies' and 'cultural border crossing' to minimise cultural bias, Aldridge and Fraser (2000) found that key elements of classroom 
observations (e.g., 'active participation') were interpreted very differently by observers in Australian and Taiwanese science classrooms. These views and results indicate that researchers gathering data in contexts involving cultures other than their own must seek and use ways to reduce cultural bias in their recorded data. Examples of suitable strategies include researchers maintaining mindfulness that their perceptions of events can be related to their own cultural background, finding ways to ensure their decision making is valid (e.g., by discussing decisions with participants and cultural advisors), and ensuring they incorporate data gathering methods which draw directly from participants.

It is widely accepted that, in research settings including cultures other than one's own, data and research quality can be enhanced through participant involvement (Bishop \& Glynn, 1999; Sieber, 1992) and many researchers have deliberately sought cultural expertise to inform their research. Banks (2006) encourages researchers to "work with people indigenous to the community who can provide them with an accurate knowledge of the perspectives, values, and beliefs within the community" (p. 196).

Examples of such practice within mathematics education include Howard, Perry, Lowe, Ziems, and McKnight (2003) who highlight important effects in their Australian study of targeted involvement of those with specific cultural expertise ('Aboriginal Education Assistants'), for example, their mentoring role (for teachers) and their ability to facilitate community links. Clark (1995a) discusses the involvement of Pasifika 'facilitators' within an initiative that improved the participation and performance of Pasifika tertiary statistics students. These students were enlisted to provide a 'cultural bridge' between the tutors and students.

Minority communities within urban settings are complex; therefore cultural advisors should be seen as informed voices from their cultural group rather than as cultural spokespeople (Cutmore \& Howard, 1995). Although researchers have identified the importance of cultural experts' advice, the roles of cultural advisors in the design of data gathering tools is less often described.

Classroom observation is an important component of much education research, preservice teacher education, and teacher development. Detailed and robust procedures for the systematic collection of observation data are well documented (e.g., Good \& Brophy, 2003); however, less attention is given to descriptions of ways to ensure the integrity of observation schedules themselves particularly in terms of 
the cultural perspectives of those being observed. Without ensuring cultural perspectives can be appropriately recorded there is potential for vital data to be misinterpreted or missed altogether, particularly as classrooms nationally and internationally are becoming increasingly culturally diverse.

This potential is magnified in classrooms with students and teachers of cultures other than those of the researchers. The context of this study is New Zealand multicultural (Māori, Pasifika, and New Zealand European) secondary school mathematics classrooms and the researcher is New Zealand European. Thus particular care was necessary in the development phase to ensure the data gathering tools would be sufficiently culturally responsive to allow all relevant data to be gathered.

For this study, literature, advice from a range of advisors, and trials were used to develop the data gathering tools ensuring the views and knowledge of many stakeholders were gathered and used. The following proverb describes the usefulness of purposefully integrating many people's skills, resources, and knowledge into one task, in this case that of designing data gathering tools for research in multicultural mathematics classrooms:

Ko tou rourou, ko tōku rourou, ka ora te iwi ${ }^{6}$

With your food basket and my food basket we will feed the people.

The data gathering tools (Appendices 3-11) were informed by the literature, the researcher's experiences in teaching and teacher education, consultation, classroom trials, and crosschecking areas of interest across tools (Appendix 12) (Figure 3.2).

\footnotetext{
${ }^{6}$ An ancient Māori (indigenous New Zealand) proverb.
} 


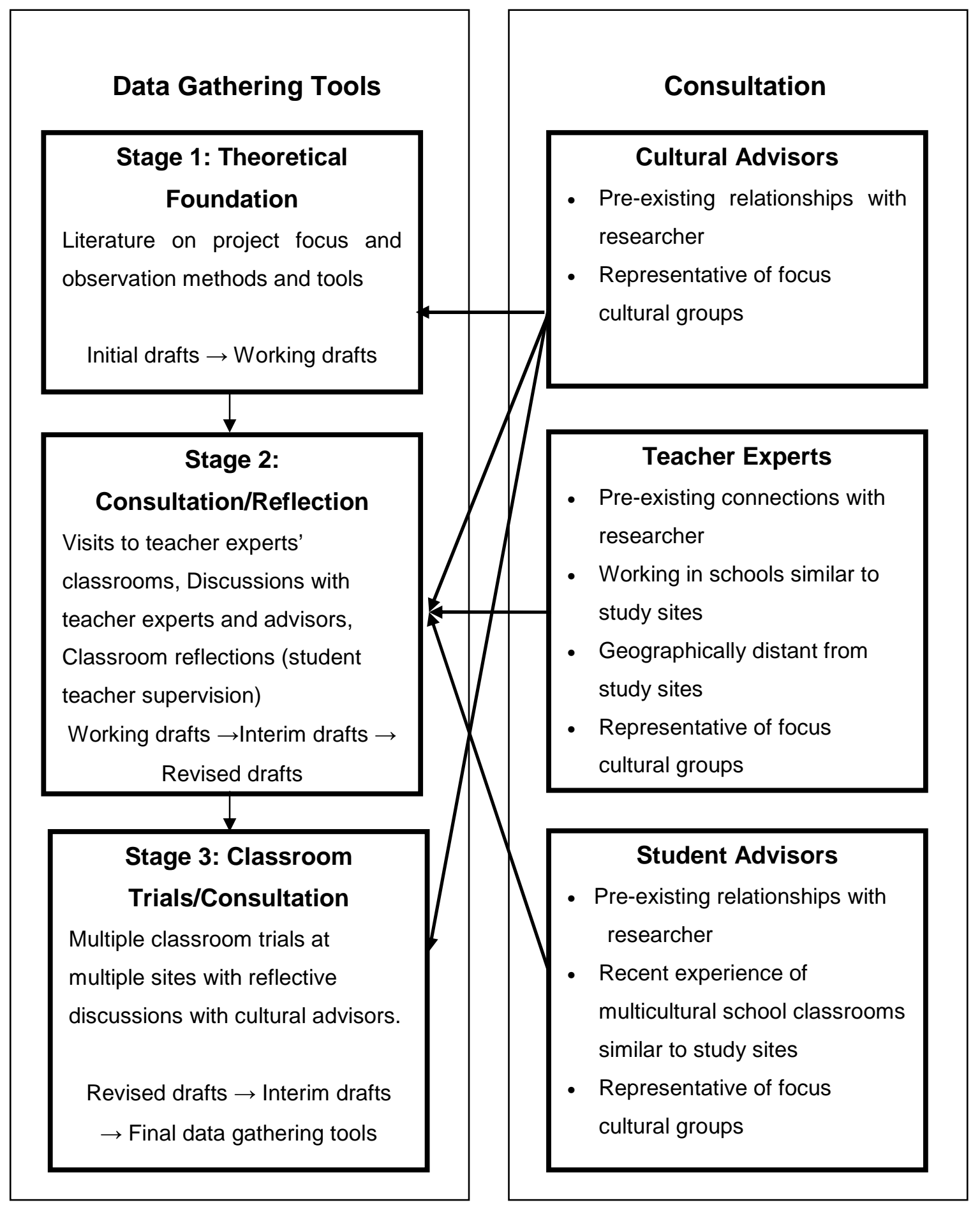

Figure 3.2: The data gathering tool development process Note: Based on table from Averill \& Clark (2007)

Initial drafts of the observation schedule, questionnaires, and interviews were compiled using key ideas distilled from a wide range of literature on teacher care (Chapter Two). Factors less frequently discussed in the literature, such as how students indicate that they have experienced aspects of teacher care, were also incorporated. 
Advisors were chosen to capture students', teachers', and cultural perspectives. The advisors' group consisted of two cultural advisors, seven student advisors, and six teacher experts. Cultural advisors were sought for their indigenous Māori and/or Pasifika cultural knowledge, expertise, and heritage and experience of New Zealand multicultural secondary school classroom contexts. In keeping with the study theme and the importance of relationships within research projects involving indigenous and minority group participants, a further consideration for advisor selection was the relationship between potential advisors and the researcher.

The cultural advisors were colleagues of the researcher and thus professional relationships already existed. These advisors carried status within their respective education communities. The Māori cultural advisor was nominated for the study by the Faculty's Te Kura Māori ${ }^{7}$ and the Faculty's Pasifika Education Committee endorsed the selection of the Pasifika cultural advisor.

The seven student advisors were volunteers from one of the researcher's classes; had recent classroom experience similar to the study students; and although their education background included negative secondary school mathematics learning experiences, they had proven to be capable mathematics learners.

In order to minimise any impact on the eventual study participants, the selected teacher experts worked in schools demographically similar to, but geographically distant from, the study sites. A mathematics education specialist, a former colleague of the researcher, was one of the teacher experts and nominated the remaining teacher experts. No pre-existing relationships existed between the remaining teacher experts and the researcher.

\subsubsection{Consultation}

Consultation regarding the data gathering tools was carried out with all advisors (cultural advisors, student advisors, and teacher experts). The views of all advisors were collected through informal semi-structured interviews. Summaries of the key ideas from each interview were returned to the advisors to check that they matched the views discussed and to invite further comments. Their input will be discussed in turn.

7 roughly translated as The School of Māori, Pacific and Indigenous Studies, which sits within the University's Faculty of Education. 
Involving cultural advisors throughout the research study rather than solely at the outset, was a deliberate act aimed at generating understanding about, engagement with, and commitment to the entire study. The cultural advisors were consulted throughout the development process. Their advice was sought on the initial drafts of the data gathering tools, revised drafts after consultation with the student advisors and the teacher experts, as the trials progressed (Figure 3.2), and during the data collection and analysis. This extended involvement ensured multiple opportunities for the cultural advisors to inform the study and for the researcher to ensure the study as a whole, and the design and use of the data gathering tools in particular, was responsive to their advice.

The cultural advisors described the benefits for research quality of the use of cultural advice for studies involving multicultural classrooms as: including safety for the researcher; increasing the depth and scope of the findings; and increasing the standing of the research:

Involving cultural advisors input [is] he whakaaro rangatira (a chiefly thought),
This is the most important stance one could make...this will help set 'safe'
guidelines for the researcher and perhaps add another dimension to the topics
being researched. Using cultural advice gives mana (standing), credibility, and
integrity to the project. Kanohi ki te kanohi (face to face) dialogue is extremely
important. (Māori Cultural Advisor)

I think it's really important, for example if you're going to be going in and you're going to be looking at multicultural [classrooms] there is no way you're going to have all that knowledge by yourself so you have to go and seek advice... because it just gives you just a little bit more insight and gives you more of a background on what you're heading into and a bit of knowledge of why and how kids are acting...the types of kids that you'll have, or the backgrounds that they come from. I see [Pasifika students in multicultural classrooms] as communities within communities. I think [cultural advice] is really important. It benefits the researcher. (Pasifika Cultural Advisor)

Particularly important additions to the observation schedule from the cultural advisors' input included methods for recording:

- how well the lesson matched Durie's (1998) "'whare tapa whā" (p. 76) model for personal health and well-being (Māori cultural advisor); and

- how the community of practice was re-established at the beginning of the lesson (Pasifika cultural advisor).

The cultural advisors' advice led to audio-taping of observed lessons to allow interpretations of specific teacher-student interactions to be sought. 
The majority of suggestions from the cultural advisors were fairly consistent with the literature on teacher care; however, further specific modifications made to the observation schedule (Appendix 3 ) following their advice included:

- sections for holistic anecdotal statements of the impact of teacher interactions (the cultural advisors' views indicated a greater interdependence of facets of caring relations than conveyed by the distinct sections of the schedule); and

- making sufficient space for quotes, anecdotal notes, and the researcher's views regarding the consequences of teacher and student actions. (Cultural advisors expressed strongly that how teachers or students displayed each component of the schedule was as or more important than its presence. For example, a checklist can record how often humour is used by a teacher but not how students appeared to interpret the humour or how it appeared to affect the classroom climate.)

Cultural advisors' suggestions also led to additions to the questionnaires and interviews. In particular, many questions were added to the third set of questionnaires (Appendices 6 and 9) in response to a discussion with the Pasifika advisor regarding achieving greater depth to the investigation of participants' views about the relevance of their culture to themselves and to their mathematics learning. Corresponding changes were made to the student and teacher questionnaires to allow comparison of teachers' and students' data.

The student advisors' responses were also fairly consistent with the literature and those of other advisors. However, the observation schedule was adapted to enable greater specificity in recording student-initiated interactions (Appendix 3 ) in response to their advice regarding the importance of teachers':

- ability to respond to students' body language:

Teachers have got to work the crowd. They have to read the kids and [when appropriate] change the activity. (Student advisor)

- knowledge of the students as individuals; and

- sensitivity with such knowledge.

Discussion of these aspects also increased the researcher's sensitivity to actions and reactions in the classroom thereby increasing the effectiveness of the use of the schedule. 
In contrast to the literature and the cultural advisors' and teacher experts' views, the student advisors were initially surprised that the observation tool included certain components (e.g., 'mathematical contexts which reflect Māori and Pasifika cultures', 'teachers sharing of themselves', and 'smiling'). However on reflection they agreed that, dependent on the teacher, these factors could be useful in developing a caring atmosphere for mathematics learning. Further, they reported that it was not important for mathematics teachers to know their students' ethnicities stating that teachers should "treat all students the same"; and that they did not all regard sustained eye contact as a positive aspect of teacher care:

I hate eye contact. You feel like they are singling you out. (Student advisor)

Subsequent to advice from the cultural and student advisors, the researcher carried out over twenty classroom visits in a wide range of school types as the visiting lecturer for student teachers' teaching practice. As a result of reflecting on these classroom experiences in light of further consultation, more modifications were made to the schedule (Appendix 3) to allow greater specificity to be recorded regarding teachers':

- explanations of their practice (e.g., "I would like you to listen to one another in the presentations because it shows and it allows us to hear and think about...." in contrast to "Be quiet");

- use of humour (from the teacher directly (related or unrelated to the lesson's mathematical content) and through the teacher using learning experiences which allowed students to incorporate their own humour);

- ways of building a sense of shared endeavour (for example using language such as "We are going to explore..." rather than "You are to copy what is on the board"); and

- sharing aspects of their personal identities.

Practices such as these are described in the literature concerning effective classroom practice (e.g., Darling-Hammond \& Bransford, 2005; Mclnerney \& Mclnerney, 2006) but appear less often in the literature on teacher care. Reflections on the classroom visits enabled them to be considered and incorporated into the study data collection.

Teacher experts were the last group to be consulted. Their opinions largely matched those gathered from the literature, other advisors, and the researcher's classroom reflections, in itself a useful reinforcement that the data gathering tools were fit for 
the purpose. However, incorporating a way to record how teachers make opportunities for students' sharing of themselves was an example of one addition to the schedule as a result of discussions with the teacher experts.

\subsubsection{Trials and crosschecking}

A working draft of the observation schedule was created using all input from the advisors and classroom reflections. It was then iteratively used and refined over ten classroom trials across four schools. Factors used to select trial classrooms included: the schools' socio-economic status and ethnic composition (to match those of the study schools); teacher skill and experience (to match those of study teachers); and the existing professional relationships between the researcher and the trial teachers.

The importance of incorporating the classroom trials in the development process is best illustrated through examples of the rationale for alterations to the observation schedule and the consequential changes (Table 3.3). The rationale for each change and suitable modifications were deliberated through an informal process including discussions between the researcher and cultural advisors and between the researcher and other teacher educators and education researchers across the trial phase.

Table 3.3: Modifications to the observation schedule as a result of classroom trials

\begin{tabular}{|l|l|}
\hline \multicolumn{1}{|c|}{$\begin{array}{c}\text { Rationale for modifying the } \\
\text { observation schedule }\end{array}$} & \multicolumn{1}{|c|}{ Additions to the schedule (Appendix 3) } \\
\hline $\begin{array}{l}\text { To be able to record further detail } \\
\text { regarding teacher care for students' } \\
\text { mathematics progress }\end{array}$ & $\begin{array}{l}\text { one-to-one teacher-student interaction } \\
\text { (mathematics content) } \\
\text { acknowledgement of students' mathematical } \\
\text { success } \\
\text { persistently maintains students' focus on lesson }\end{array}$ \\
\hline $\begin{array}{l}\text { To explore possible links between } \\
\text { teacher care and class engagement }\end{array}$ & $\begin{array}{l}\text { recording the proportion of the class highly } \\
\text { engaged at four roughly equally spaced points } \\
\text { through the lesson } \\
\text { recording whether or not the class was engaged } \\
\text { to (apparent) teacher expectations }\end{array}$ \\
\hline $\begin{array}{l}\text { To include a further measure of teacher } \\
\text { care for individual students }\end{array}$ & $\begin{array}{l}\text { one-to-one teacher-student interaction } \\
\text { (unrelated to the lesson's mathematics content) }\end{array}$ \\
\hline $\begin{array}{l}\text { To include a measure of the quality of } \\
\text { teacher-student relationships }\end{array}$ & $\begin{array}{l}\text { student-initiated interactions with the teacher } \\
\text { (e.g., use of humour, asking questions) }\end{array}$ \\
\hline $\begin{array}{l}\text { Completed observation schedules for } \\
\text { different lessons for the same teacher } \\
\text { could vary greatly depending on the } \\
\text { nature of the lesson and learning } \\
\text { experiences. }\end{array}$ & $\begin{array}{l}\text { a brief overview of the topic and style of the } \\
\text { lesson }\end{array}$ \\
\hline
\end{tabular}


The classroom trials also led to practical data gathering decisions important for the eventual consistency and quality of the study data. For example, decisions regarding the start and end time for the observations could be considered, trialled, and made. Some trial teachers required students to line up outside the classroom prior to the lesson and therefore for these teachers the first interactions with students were conducted outside the classroom. For others, the first interactions were as the students arrived into the classroom from their previous lessons. The trials showed that despite the importance of capturing the initial teacher-student interactions, data gathering outside the classroom before the lesson was comparatively intrusive and therefore less likely to generate representative data. For consistency in approach across all study observations, it was decided the data collection period would begin when students first entered the classroom.

Similarly, it became apparent that data collected during the minutes in the classroom after the lesson had concluded were also important as it is often in this time that students initiate informal conversations with the teacher, offer farewells, questions, comments, and use humour. Therefore each observation continued until all students had left the classroom. Exceptions to this pattern were made when teachers held students back for disciplinary discussions at which times student privacy was given precedence over data collection.

Other examples of changing the schedule through the classroom trial phase which allowed further specificity in data to be obtained included: recording acknowledgement of 'effort' as well as of 'mathematics success'; recording whether students' questions were about or unrelated to mathematics; and indicating the level of application of observation aspects (Appendix 3).

Very few events were observed during classroom trials in the area of teacher care for students as culturally located individuals and hence rather disappointingly there was little opportunity to revise this section of the schedule. This difficulty highlights the importance of ensuring trial classrooms can provide the necessary environments to inform all aspects of the schedule.

Trialing of the draft student and teacher questionnaires and interviews was also carried out within the six months immediately preceding initial data collection. The general themes and specific questions of the questionnaires and interviews (Appendices 4-11) were drawn from theory described in Chapter Two. Questions adapted from those used 
within the 'Tripod Project' (which explores similar themes in the area of teaching care for students as individuals) were included in questionnaires where suitable.

The first student questionnaire (Appendix 4) and all student interview questions (Appendix 10) were trialed with Year 9 classes at a school similar to the study schools. As trialing was carried out late in the school year, Year 9 students were chosen rather than those then finishing Year 10, as they were closer to the stage the study students would be at the beginning of data collection. An iterative process of trial and refine was used. In each iteration one entire class completed the questionnaires; two individual students from the class filled out their questionnaires voicing their thinking to the researcher as they responded, in order that the researcher could enhance the content and face validity of the questions (S. Fontaine, personal communication, 16 May, 2005); and the same two students then responded to the interview questions. This process was repeated twice more with different classes within the same school over a two week period. The main changes made to the questionnaires and interviews were clarifying wording of questions and including further options in questions using lists.

Teacher questionnaires (Appendices 7-9) and interviews (Appendix 11) were trialled with teachers geographically distant to the study in order to avoid exposure of the study teachers to the questions before the study began. Again, questionnaire responses were voiced to the researcher allowing issues of clarity and relevance to be identified. Again three cycles were used to ensure the questionnaires and interview questions were fit for the purpose.

After all trials were complete, all data gathering tools were discussed and refined using the trial results and the advice of the cultural advisors and mathematics education colleagues. A complete crosscheck was carried out to ensure that relevant data for all of the key aspects of the study were being gathered across each of the three data collection methods (Appendix 12). As a result of the crosscheck further questions were included in the third student questionnaire to ensure a fuller set of data could be gathered on students' perceptions of barriers to their learning and of their teachers' use of real life contexts. Consistency of coverage was confirmed through the crosscheck process providing confidence that the study had a sound methodological triangulation foundation (Denzin, 1985). All student questionnaires were then translated into Māori and Samoan (Appendices 4-6) enabling students proficient in those languages to select whether to use the

\footnotetext{
${ }^{8}$ Available at http://www.ksg.harvard.edu/tripodproject/ (retrieved June 16, 2008)
} 
translated or the English-language questionnaires. To maximise the integrity of the translations, the translators were nominated as experts by the respective cultural advisors and were fluent speakers of te reo Māori and Samoan respectively. A further check on the suitability of the translations was provided by the cultural advisors.

Further additions were made to the second and third sets of questionnaires and interviews during the first year of the study in response to data gathered earlier in the study, further reading, and consultation with cultural advisors. The observation schedule and the questionnaires remained constant for the second year of the study to facilitate comparison of data across both years.

\subsubsection{Discussion}

The evidence of changes made to the data gathering tools due to all development stages as described in Section 3.2 indicates the importance of using a range of ways to inform the development of rigorous data gathering tools that can be used with confidence for multicultural classroom contexts, and indicates that development by theory alone or through a combination of theory and trials may not provide tools fit for the purpose. Every aspect of the development process contributed to the final form of the observation schedule, questionnaires, and interviews thus enabling further detail of a broader range of relevant data to be captured including aspects less well documented in the literature. Use of such a process is important in order to enhance the credibility of findings particularly amongst teachers and researchers involved with, and of, indigenous and minority cultures, often the very groups performing less well within traditional achievement measures and therefore those for which we most need suitable research data, analysis, and findings.

Rigorous development of data gathering tools can place considerable demands on timeframes and budgets. However, quality research is dependent on quality data and the use of a comprehensive process to ensure data gathering tools allow the full scope of relevant data to be captured and to sensitise the researcher/s to the research context is essential. For small or large-scale classroom-based investigations in multicultural contexts attention must clearly be paid to seeking and integrating stakeholders' (in this instance, students, teachers, and cultural experts) skills and knowledge. 


\subsection{Ethical considerations and study participants}

This section describes ethical considerations of the study design and implementation and participant selection. Full ethical approval for this study was given by the Victoria University of Wellington Human Ethics Committee. Because the study was to include Māori and Pasifika participants, methods consistent with Māori-centred research (Cunningham \& Durie, 1998) and the Pasifika Education Research Guidelines (Anae et al., 2001) were used in the research design and implementation. Specifically, emphasis was given to:

- integrating the values of reciprocity (e.g., providing summaries of collected data), humility, service (e.g., disseminating findings), and respect (e.g., seeking participants' permission to tape record interviews) (Anae et al., 2001) into the study design and implementation;

- establishing contact with student participants and their parents (for student consent and parental consent) through "a common person" (Anae et al., 2001, p. 37), their classroom teachers;

- building appropriate levels of rapport with participants;

- acknowledging oral language as a central means of sharing information (e.g., using face-to-face interviews);

- incorporating ways to be sensitive to and record messages conveyed using body language (e.g., through consultation and lesson observations);

- incorporating Māori and Pasifika cultural advice at all stages of the study;

- providing the student questionnaires in te reo Māori and Samoan (the origin language of the highest proportion of Pasifika study students);

- adopting a dynamic and flexible research process (Anae et al., 2001) (e.g., modifying the research tools during the study);

- ensuring confidentiality of all responses;

- giving food in acknowledgement of participants' freely-given data (at the conclusion of the data gathering with each class); and

- having a clear focus on disseminating the study findings to inform those involved in teaching and learning of Māori and Pasifika students (e.g., as a pre-service teacher educator and academic).

In selecting the study participants, the study schools were determined first, followed by selection of the study teachers for the initial year of data collection. After all data were 
gathered from the first year of the study, negotiations with the initial study teachers and their colleagues determined the study teachers for the second year of data collection. The study classes were selected as the class of each study teacher that most closely matched the study target group (i.e., average to above average Year 10 students). In order to ensure all target groups (Māori, Pasifika, and New Zealand European students) would be included, "purposive sampling" techniques (Denscombe, 2001, p. 15; Kemper, Stringfield, \& Teddlie, 2003; Merriam 2001) were chosen as those most appropriate for selecting the study schools.

For reasons of practicality and manageability it was decided to use three study schools in the greater Wellington city area. Consistent with purposive sampling, further parameters for the selection of study schools were determined to facilitate data collection that would most effectively address the research questions in a way relevant for many New Zealand multi-cultural city schools and students. Consideration was given to school size, ethnic composition, and inclusion of both boys and girls. The initial decisions were that study schools must have:

- at least 500 students (to ensure sufficient Year 10 students in the sample);

- at least 20\% Māori, 20\% European and 20\% Pasifika students (to ensure sufficient Māori and Pasifika students for validity of the study); and must be

- co-educational state secondary schools (consistent with the majority $(71 \%)$ of New Zealand state secondary schools (251 of 351) (Education Counts, 2007).

Using these criteria, three possible sample schools were identified using information included in the most recent Education Review Office school review reports. The Heads of Department: Mathematics of these schools were approached in April 2005 regarding involvement in the project. Two schools agreed and the third declined due to an impending change of Head of Department.

In order to gain a full complement of study schools, four further schools were approached: three which closely matched the original parameters; and one further school (very large, single sex, comparatively high socio-economic and monocultural) was approached as a contrast to the two existing study schools in case all three other schools declined involvement. Three of these schools including the contrast school agreed to be involved in the study. One school declined due to workload pressures. 
Of the five willing schools the three that most closely matched the parameters originally determined were selected to be the study schools (subsequently referred to as Samasama, Whero, and Uliuli) (Table 3.4). The remaining two schools were used to trial the data gathering tools and as back-up schools in case of the withdrawal of a study school.

Table 3.4: Study schools ${ }^{9}$

\begin{tabular}{|l|l|l|l|}
\hline \multicolumn{1}{|c|}{ School } & \multicolumn{1}{|c|}{ Samasama } & \multicolumn{1}{c|}{ Uliuli } & \multicolumn{1}{c|}{ Whero } \\
\hline Decile ${ }^{10}$ range & $1-3$ & $1-3$ & $1-3$ \\
\hline Type & $\begin{array}{l}\text { Integrated } \\
\text { educational }\end{array}$ & St- & State co-educational \\
\hline $\begin{array}{l}\text { Number of } \\
\text { students }\end{array}$ & $500-600$ & $900-1100$ & $500-600$ \\
\hline $\begin{array}{l}\text { Students' } \\
\text { gender }\end{array}$ & $\begin{array}{l}\text { Approximately 55\% } \\
\text { male and 45\% female }\end{array}$ & $\begin{array}{l}\text { Approximately 60\% } \\
\text { male and 40\% female }\end{array}$ & $\begin{array}{l}\text { Approximately 55\% } \\
\text { male and 45\% female }\end{array}$ \\
\hline $\begin{array}{l}\text { Students' } \\
\text { ethnicities }\end{array}$ & $\begin{array}{l}\text { Over 20\% New Zealand } \\
\text { European } \\
10-20 \% \text { Māori } \\
50-60 \% \text { Pasifika } \\
\text { (predominantly } \\
\text { Samoan, with less than } \\
10 \% \text { of each of Cook } \\
\text { Island Māori, and } \\
\text { Tokelauan) } \\
\text { Less than 10\% Asian }\end{array}$ & $\begin{array}{l}\text { Over 30\% New Zealand } \\
\text { European } \\
20-30 \% \text { Māori } \\
30-40 \% \text { Pasifika } \\
\text { (predominantly } \\
\text { Samoan, with less than } \\
10 \% \text { of each of Cook } \\
\text { Island Māori and } \\
\text { Tokelauan) } \\
\text { Less than 10\% Asian } \\
\text { Less than 5\% Other }\end{array}$ & $\begin{array}{l}\text { Over 20\% New Zealand } \\
\text { European } \\
50-60 \% \text { Māori } \\
20-30 \% \text { Pasifika } \\
\text { (predominantly } \\
\text { Samoan, with 5\% of } \\
\text { Tokelauan and other } \\
\text { Pacific groups) } \\
\text { Less than 5\% Asian }\end{array}$ \\
\hline
\end{tabular}

Data were collected over two years: Phase One (2006) and Phase Two (2007). Negotiation regarding schools' involvement including which teachers would be the study teachers was carried out in each case with the Head of Department: Mathematics. Two initial criteria were used to select Phase One study teachers. Teachers must be teaching a class that included students within the study target group (average to above average Year 10 students) and be willing to be involved. Consistent with "unique sampling" (Merriam, 2001, p. 62) sometimes called "extreme case sampling" (Kemper et al., 2003, p. 280) a further criterion for participant teachers in Phase One was that they must be able to provide examples

9 Based on data taken from the most recent Education Review Office reports (as at mid2005, the time of participant selection) retrieved from http://www.ero.govt.nz/ero/publishing.nsf/Content/Home+Page.

To disguise the identity of study schools, specific reports are not referenced and ranges of values and rounding have been used.

10 A measure of the comparative socio-economic status of the school and its local community

11 A school, previously private, but now integrated within the state-funded education system

${ }^{12}$ The nomenclature of the ethnicity groups is widely accepted practice in official New Zealand documents and is as recorded in official school reviews (Education Review Office). 
of very good practice (i.e., have well managed classrooms where effective teacherstudent relationships were evident) in order that the sample cases would best illuminate the research questions.

Each Head of Department was invited to identify a teacher in their department who would be suitable for the study. For Phase One, in each case they self-nominated (SS ${ }^{13}, \mathrm{WW}$, and UU at Samasama, Uliuli, and Whero respectively).

Towards the end of Phase One, discussions were held with the participant teachers regarding their school's involvement in Phase Two. Three options were offered: withdrawal of their school from the study; continuing in the study with the same teacher and a new Year 10 class; continuing in the study with a new teacher or teachers who, consistent with "snowball sampling" (Merriam, 2001, p. 63; Kemper et al., 2003, p. 283), would be nominated by the school's Phase One teacher. Snowball sampling uses informants with insider knowledge to identify cases likely to maximise the chances of illuminating the research questions.

Samasama elected to continue with the study with two teachers new to the study (ST and SG). SS identified the teachers as potentially benefiting from the professional development possible through involvement and the teachers both indicated independently to SS and the researcher that they would be happy to be involved. A compromise had to be made regarding the year level of the Phase Two Samasama study classes as neither ST nor SG were to teach Year 10 classes in 2007. In each case, the Phase Two Samasama study classes were to be Year 9 classes. However, when Phase Two data collection commenced the researcher decided that classroom management problems made SG's classroom unsuitable for the study and therefore data gathering was not continued with SG or her class.

Uliuli did not initially pursue involvement in Phase Two; however, UU agreed to students in her Year 10 class during the Phase Two year being involved in Phase Two interviews and questionnaires. The researcher and UU jointly agreed that classroom observations, teacher questionnaires, and interviews would not be used again with her, as they would not be likely to differ greatly from her Phase One data. This arrangement was changed in the week before school began in Term 1 as

13 The codes indicate the study school (first letter of the code) and the teacher (second letter of the code), e.g. SS and ST were the two teachers at Samasama school, WW and WK were the two teachers at Whero school, and UU and UM were the two teachers at Uliuli school. In each case the double letter named teacher participated in the study in 2006 and the teachers with unlike letters (ST, WK, and UM) participated in 2007. 
another Uliuli teacher (UM) indicated interest in being involved in the study. UM's involvement removed the need to collect data from UU's 2007 class.

Whero chose to continue in Phase Two with a new teacher (WK). Of the three Phase Two teachers, two were in their third year of teaching (ST and UM) and the other was the assistant head of the mathematics department (WK) (Table 3.5).

All study classes included between twenty-one and thirty students (Table 3.5). Altogether there were 161 students in the study classes across Phase One and Phase Two: 49\% female, 51\% male, 29\% Māori, 32\% Pasifika, 32\% New Zealand European, and $5 \%$ Other. $^{14}$

\section{Table 3.5: Summary of study participants}

Note: Percentage values have been rounded to disguise the identity of study participants.

\begin{tabular}{|c|c|c|c|}
\hline School & Samasama & Whero & Uliuli \\
\hline \multirow{2}{*}{$\begin{array}{l}\text { Phase } \\
\text { One } \\
\text { teachers } \\
\text { and } \\
\text { study } \\
\text { classes } \\
\text { (2006) }\end{array}$} & $\begin{array}{l}\text { SS } \\
\text { Experienced teacher } \\
\text { Māori/New Zealand } \\
\text { European female }\end{array}$ & $\begin{array}{l}\text { WW } \\
\text { Experienced teacher } \\
\text { New Zealand European } \\
\text { male }\end{array}$ & $\begin{array}{l}\text { UU } \\
\text { Experienced teacher } \\
\text { New Zealand European } \\
\text { female }\end{array}$ \\
\hline & $\begin{array}{l}\text { Able Year } 10 \text { class } \\
25 \text { students } \\
\\
70 \% \text { female } \\
30 \% \text { male } \\
\\
5 \% \text { Māori } \\
50 \% \text { Pasifika } \\
30 \% \text { New Zealand } \\
\text { European } \\
12 \% \text { Other }\end{array}$ & $\begin{array}{l}\text { Able Year } 10 \text { class } \\
21 \text { students } \\
\\
40 \% \text { female } \\
60 \% \text { male } \\
\\
60 \% \text { Māori } \\
25 \% \text { Pasifika } \\
15 \% \text { New Zealand } \\
\text { European } \\
0 \% \text { Other }\end{array}$ & $\begin{array}{l}\text { Mixed ability Year } 10 \\
\text { class } 30 \text { students } \\
50 \% \text { female } \\
50 \% \text { male } \\
\\
25 \% \text { Māori } \\
35 \% \text { Pasifika } \\
35 \% \text { New Zealand } \\
\text { European } \\
7 \% \text { Other }\end{array}$ \\
\hline \multirow{2}{*}{$\begin{array}{l}\text { Phase } \\
\text { Two } \\
\text { teachers } \\
\text { and } \\
\text { study } \\
\text { classes } \\
\text { (2007) }\end{array}$} & $\begin{array}{l}\text { ST } \\
\text { Early career teacher } \\
\text { First generation New } \\
\text { Zealander }\end{array}$ & $\begin{array}{l}\text { WK } \\
\text { Experienced teacher } \\
\text { New Zealand European } \\
\text { male }\end{array}$ & $\begin{array}{l}\text { UM } \\
\text { Early career teacher } \\
\text { First generation New } \\
\text { Zealander }\end{array}$ \\
\hline & $\begin{array}{l}\text { Mixed ability Year } 9 \text { class } \\
29 \text { students } \\
45 \% \text { female } \\
55 \% \text { male } \\
\\
10 \% \text { Māori } \\
55 \% \text { Pasifika } \\
30 \% \text { New Zealand } \\
\text { European } \\
3 \% \text { Other }\end{array}$ & $\begin{array}{l}\text { Able Year } 10 \text { class } \\
23 \text { students } \\
\\
45 \% \text { female } \\
55 \% \text { male } \\
\\
60 \% \text { Māori } \\
20 \% \text { Pasifika } \\
15 \% \text { New Zealand } \\
\text { European, } \\
0 \% \text { Other }\end{array}$ & $\begin{array}{l}\text { Able Year } 10 \text { class } \\
30 \text { students } \\
40 \% \text { female } \\
60 \% \text { male } \\
\\
30 \% \text { Māori } \\
10 \% \text { Pasifika } \\
60 \% \text { New Zealand } \\
\text { European } \\
7 \% \text { Other }\end{array}$ \\
\hline
\end{tabular}

This study is concerned with effective classroom relationships. Care was actively and consistently taken by the researcher to acknowledge and foster relationships

${ }^{14}$ Ethnicity data taken from school roll information 
with research participants in order to reflect this theme at all stages of the project and maximise the quantity and quality of the data. The researcher had existing collegial relationships with all of the Heads of Department approached for their school's involvement. This facilitated communication and rapport with them and through them, with other involved teachers at their schools.

Meetings with Phase One teachers were carried out in July and August of the year preceding data collection. The purpose of these meetings was to provide information about the project including the project focus and data gathering methods, discuss consent procedures, and to set up classroom visits (two for each classroom) prior to commencing Phase One. Observation can affect teachers' practice (Angrosino \& Mays de Perez, 2000; Good \& Brophy, 2003; Merriam, 2001; Wragg, 1999) and none of the study teachers had previously been involved in classroom research or had been consistently observed by another teacher. The classroom visits were intended to:

- help minimise the eventual impact of researcher presence on teacher practice;

- further refine the study focus and the content and design of the data gathering tools;

- immerse the researcher in classroom environments of experienced teachers working with Year 10 multicultural classes; and

- further develop researcher-teacher rapport.

All teachers subsequently reported comfort regarding the classroom visits indicating they assisted them in becoming used to being observed. It was therefore hoped that teachers were less likely to modify their classroom behaviour when data gathering commenced.

Similar meetings to provide information about the project were held with Phase Two teachers in the final week of Term 4, 2006 (ST and WK) and the week preceding Term 1, 2007 (UM). The Phase One teachers had all discussed the project with the Phase Two teachers from their schools and as a result the offer of classroom visits prior to data collection was made but not felt necessary by the Phase Two teachers or the researcher.

The initial teacher interviews were held in the week prior to the first set of classroom observations by which time the teachers knew which students would be in the study classes. At this stage teachers were able to become re-familiarised with the practicalities of the project and have last minute questions answered before 
observations began, potentially increasing their comfort levels and therefore the quality of study data.

All aspects of the data gathering and feedback were negotiated with the teachers. This was generally managed via email or telephone conversations in the weeks before the data gathering rounds, and by email and mail for consultation over conference and other publications ensuing from the study. Feedback was provided to all study teachers (Section 3.4.1). The researcher ensured that all data gathering, feedback sessions, timing, and processes were convenient for the teachers. Similarly, communication with school principals (for information giving, consent, and sharing of data summaries, draft publications etc.) and parents (for information giving and consent) was carried out through the study teachers. These decisions regarding researcher-participant interaction were intended to show respect for the teachers and give them shared control over how the study may impact on their teaching and lives.

Written information about the project and consent forms (Appendix 13) were distributed to Phase One teachers in Term 4, 2005 and to Phase Two teachers in Term 1, 2007. The study teachers agreed to manage the consent process within the school as they had established rapport with students, parents/guardians, and principals.

Four of the teachers (WW, UU, ST, and UM) requested that the researcher explain the study and consent process to the students and this was done. WW then talked through the information sheet with the class, so that they could explain it to their parents. The classes with the greatest proportion of student participation had teachers who expressed their view that both the research project and returning the forms were important. WW believed that the reason for the lack of consent for two of his students was their parents' lack of ability with English and therefore ability to read the information letter and consent form. This may also have been a contributing factor for lack of consent for some students in other classes.

Teachers' unfamiliarity with formal consent procedures was evident in Phase One. For example, SS initially stated to her class that she required all forms to be signed and returned. Following further explanation by the researcher of consent procedures including a request that students not be compelled to consent, SS did not enforce this. UU offered incentives of lollipops for returning the signed forms. Despite this incentive, class UU had one of the poorest return rates in the study (41\% compared with $92 \%, 91 \%, 38 \%, 70 \%$, and $53 \%$ for classes SS, WW, ST, WK, and UM respectively). Students for whom consent was not received did not participate in 
interviews, were invited to sit at desks distant from the audio-recorder, and completed questionnaires anonymously if they asked the researcher if they could participate. Observational data did not identify non-consent student responses or behaviours.

\subsection{Data gathering}

This section describes the practicalities and details of the data collection methods by providing an overview and details of each method in turn. For each study class data were gathered by means of:

- teacher interviews prior to the start of Term 1;

- eight or more lesson observations, student and teacher questionnaires, and student and teacher interviews (in the first four weeks on Term 1);

- four lesson observations, student and teacher questionnaires, and student and teacher interviews (late Term 2); and

- four lesson observations, student and teacher questionnaires, and student and teacher interviews (early Term 4 for Phase 1, late Term 3 for Phase Two).

The total data set included 100 lesson observations, 339 student questionnaires, 18 teacher questionnaires, 18 sets of student interviews (involving a total of 138 student interviews), and 24 teacher interviews.

In order to maximise the comparability of data across the two years of data collection, the observation schedule and questionnaires were identical and the interview questions were close to identical across Phases One and Two. The observation schedule (Appendix 3) was used for all lesson observations. Different questionnaires and different sets of interview questions were used in the first, second, and third rounds of data collection for students and for teachers (Appendices 4-11).

Methods used to enhance the validity, reliability, and consistency of the data included:

- repetition across schools and across Phases One and Two;

- repetition across questionnaires and interviews; and

- triangulation of data collection methods (observations, interviews and questionnaires). 
The observation schedule was used to record teacher and student behaviours, brief quotes and descriptive anecdotal notes. Minor timetable clashes between study classes caused a small number of early Term 1 lessons to be missed. When clashes did occur, decisions regarding which lessons to observe for each class were based on three criteria: achieving a balance between observing morning and afternoon lessons; observing sequences of at least three consecutive lessons; prioritising early lessons of classes new to their teacher. Timetable clashes were avoided for the second and third sets of observations in both Phases of the study by selecting different weeks for observing lessons with each class. ${ }^{15}$ Two processes were used at the end of each observed lesson to ensure comparability of recording between lessons within and across data gathering rounds: a brief written overview of the lesson was completed; and the entire schedule was reviewed as a final check that recorded data were representative of the lesson.

An audio recorder was set up unobtrusively at a front corner of each classroom to record teacher/whole class interactions. Whenever possible, before the class arrived the recorder was in place and the researcher was in a seat at a back corner of the classroom. At the end of the lesson the researcher remained in place until most of the class had left thereby keeping the researcher's presence as unobtrusive as possible and limiting researcher interactions with students and teachers. Exceptions included when the lesson prior to the observed lesson went over time (fewer than five lessons in total) and when teachers were having discipline-related discussions with individuals (over half the lessons for SS, seldom for the other teachers).

In an attempt to avoid the audio recording altering normal classroom practice, four lessons were not recorded: in three cases this was for the initial lesson observations, in an attempt to enhance the comfort levels of the teachers; in the other case several students had been altering their normal classroom practice to speak directly into the recorder during class. Removing the recorder for one lesson was effective in stopping this.

Written lesson observation data were summarised by theme and holistically (Chapter Five). Audio recordings were saved but not transcribed in full. Components

15 Observed lessons: SS, 8 of the first 9; UU, 9 of the first 10; WW, 8 of the first 16 (including 3 of the first 5); ST, 8 of first 11; UM, 9 of first 13; WK, 9 of first 14 (including 5 of the first 7).

WW and WK had taught the study classes in the year immediately prior to the study and therefore in these classrooms the teacher-student relationships were already established. 
of the lesson, teacher behaviours, and teaching strategies were analysed from the audio files and sections were transcribed as appropriate (Chapter Five).

The teacher questionnaires were completed following each set of classroom observations. No questions of clarification were asked regarding the teacher questionnaires. Student questionnaires were generally carried out in the lesson immediately following the final lesson observation of each set. In two cases, due to school-based and researcher-based logistical constraints, they were completed in the final ten minutes of the last observed lesson. This was not ideal as it placed a time constraint on completion. Students were asked to complete the questionnaires without discussing the questions and in almost all cases complied with this request.

The three student questionnaires were completed by 96,107 , and 136 students respectively. After each class had completed each student questionnaire, teachers were left with spare questionnaires and postage-paid addressed envelopes in an attempt to collect completed questionnaires from students absent from class; however, only two catch-up questionnaires were received.

Students raised very few questions of clarification when answering the questionnaires. Two students who had completed their questionnaires in Samoan asked who would be translating their answers and another student asked for clarification of what was meant by one of the options given for a question in Questionnaire One (whether or not 'cultural activities' referred to their in-school or out-of-school activities or both). The corresponding question in Questionnaires Two and Three was subsequently refined to distinguish between these possibilities.

When discussing the summarised data from the first two student questionnaires, both cultural advisors suggested trying to investigate further the apparent contradiction between data on students' responses regarding the importance to them of their culture and whether or not they want their teachers to:

- know their ethnicity;

- know about the worlds of Māori and Pasifika people; and

- use these contexts in their teaching.

Questions were added to the third student questionnaire (Appendix 6) in an effort to further illuminate these areas. 
Student questionnaire data were stored within and analysed using SPSS due to the quantity of data. With only six sets of teacher questionnaires, analysis of teacher questionnaire data was carried out manually.

The timing of all of the interviews was negotiated and interviews were held in locations selected by teachers: for teacher interviews, in their offices with SS and WW and in the school interview room or an empty classroom (ST, WK, UU, UM); for student interviews, in SS's office for SS's class and in nearby empty classrooms or resource rooms for all other classes. With the exception of UM, teachers liaised with school staff when necessary for booking interview rooms and times for students to be out of other teachers' classes. The researcher booked the rooms for interviewing students from UM's class.

Prior to each interview teachers and students were told the approximate length of the interview, the number of interview questions, and their permission to audiotape responses was sought. The interviews (Appendices 10 and 11) were semistructured allowing further detail to be explored.

In an attempt to maximise the quality of students' interview data, four strategies intended to help students feel comfortable about being interviewed were used:

- The first set of student interviews was carried out after the researcher had observed at least eight complete lessons in their classroom and the students appeared comfortable with the researcher's presence. By then the researcher's commitment to the study had already been demonstrated and students and researcher had shared experiences of their mathematics classroom that aided the interview discussions.

- Students had been encouraged to give open and honest replies. In each classroom, either the teacher or the researcher or both had explained the project, confidentiality, and the importance of speaking honestly. In order to enhance students' confidence that their responses would be anonymous they were not asked to identify themselves in the first set of interviews. A cost of this decision is that some interview responses are unable to be linked to students' ethnicities or to data gathered in other ways.

- Questionnaires were completed before the interviews so students knew the types of questions they were likely to be asked.

- Having had the option of answering questionnaires in Samoan or Māori it was hoped students of these ethnicities would have increased comfort. 
However, in spite of these factors, the initial set of student interviews (Uliuli) was disappointing. Through these interviews students often gave one-word answers and did not give clear reasons or full explanations. On reflection, the researcher felt that the method of selecting students for the interviews was at fault. Students had been selected in pairs by UU. The researcher felt that the students had not necessarily felt comfortable talking in front of one another and additionally they may not have felt that they had valuable things to offer. Therefore in all subsequent student interviews the following strategies were used to gain more substantive responses:

- Students were invited rather than required to be interviewed and were able to choose to come to the interview on their own or with another student in all interviews. A total of 138 students were interviewed: 56 individually and 82 in pairs.

- Students were told at the beginning of the interview that their responses were important and would be valuable and useful.

All subsequent student interviews produced more useful and detailed responses.

Some interview questions that used the word 'you' or 'your' created an unanticipated dilemma in that they generated two types of student response depending on whether the student had interpreted 'you' or 'your' in the singular or the plural form. For example:

How important to you is it for your teacher to know about your cultural identity?

Does your teacher care about your maths progress?

How does your teacher encourage you?

Subsequently students were asked to clarify whether they were responding from an individual or whole class perspective.

All interviews were transcribed and transcriptions were checked with the original tapes and corrected where necessary.

\subsubsection{Participant feedback}

Teacher participants were given feedback about the study through the researcher:

- providing and discussing summaries of student questionnaire data;

- providing and discussing the observation schedule;

- seeking their feedback on draft journal articles and conference papers; and

- providing final versions of journal articles, conference papers, and other writing drawn from the study. 
The student participants and principals received feedback about the study through the study teachers. Questions exploring the impact on opinions or behaviour of having shared study feedback were included in the third round of interviews and questionnaires.

In both Phases One and Two, summaries of responses across all classes from Student Questionnaires One and Two (combined) and from Questionnaire Three (Appendices 4-6) were shared with teachers and subsequently with students. In each case sharing was carried out in the fortnight following the second and third rounds of data gathering. The teachers reported finding the summaries interesting in terms of: what had been asked; the student responses; and the areas of greatest change between the data gathering rounds.

As the summaries combined data from all study classes but did not identify which results came from each school, students could feel confident that their personal responses and those of their class group were included but anonymous. Students' interest in the summaries varied from none at all to moderate interest. Their reactions to the summarised data provided a further check on the validity of the data. These included some student interest and a general lack of surprise at the results, and through their discussions indicating general acceptance of the summary as being representative of their and their peers' thoughts and feelings. A small number of results which surprised some students included that some students wanted sweets as rewards and that one student felt that because they were "white" they did not have a culture.

Teachers were offered a copy of the observation schedule (i.e., a clean copy with no observational data) after the second round of data gathering and were able to discuss the schedule with the researcher. All teachers were interested in the schedule and reported that they did not know whether or not they did some of the things being observed (consistent with discussion in Good \& Brophy, 2003). SS requested that the schedule be shared with the school staff in a professional development meeting once the study was complete as she felt it would be useful for the school staff to use to consider their own practice. This was taken as affirmation of the pertinence of schedule content.

A further means of feedback to participants included that participant teachers were offered copies of draft and final versions of journal articles and conference papers drawn from the study data both as study feedback and to incorporate their 
perspectives on the data analysis and reporting. All were interested in receiving these but few responses were offered. Although generous timeframes were allowed for feedback, at times the reason given for not giving feedback included lack of time, an indication of the demanding nature of classroom teaching. Responses received were affirming of the articles and papers. Again their reactions of apparent comfort with the findings can be regarded as helping confirm the validity of the data and analysis.

\subsection{Data analysis and description}

The two main rationales for mixed method data analysis include considerations of representation and legitimation (Onwuegbuzie \& Teddlie, 2003). Mixed methods research allows greater representation within analysis through triangulation, complementarity (clarifying results obtained by one method through using another method), development (using results from one method to inform another method), and expansion (expanding the scope of an inquiry by use of different inquiry methods for different components). Onwuegbuzie and Teddlie argue that mixed methods research offers a more comprehensive means of legitimising findings (ensuring the validity, trustworthiness, credibility, as well as the confirmability and transferability of findings) than qualitative or quantitative methods alone. "Legitimation" can be enhanced through descriptive, interpretive, theoretical, and evaluative validity and generalisability of findings (p. 354).

A mix of "variable-oriented" and "case-oriented" approaches was used in both data gathering and data analysis (Onwuegbuzie \& Teddlie, 2003, p. 363) in order to gain optimal understanding of specific teacher behaviours while also maintaining a holistic perspective of teacher care. Consistent with qualitative analysis approaches (Grbich, 2007; Patton, 2002) informal reflective summaries of qualitative and quantitative data were recorded throughout the study and were used to inform the formal analysis. Formal analysis of qualitative and quantitative data was carried out separately ("parallel mixed analysis", Onwuegbuzie \& Teddlie, 2003, p. 365) in order to maintain the integrity of, and acknowledge the assumptions underlying, the inferences gained from each method.

Analysis within ethnographical research carried out in educational settings usually involves categorising the data into categories and sub-categories that emerge from the data (Merriam, 2001). Data are arranged into the categories until saturation occurs and no further change to categories results from including analysis of further 
data. Relationships between the categorisations can be shown using grids, tables, diagrams, flow charts, etc. in order to provide a "cognitive map" (Werner \& Schoepfle, 1987, p. 24) to illustrate findings.

For this study categorisation was carried out after all of the data were gathered. Two main sources of typology were used: "interpretive" or "deductive" (constructed from pre-existing analytical concepts) and "investigative" or "inductive" (created by the researcher) (Onwuegbuzie \& Teddlie, 2003, p. 370; Patton, 2002, p. 453) (Table 3.6). Interpretive approaches were used in order to link new understandings arising from the study data into several existing analytical frameworks (Averill, Te Maro et al., 2009; Bishop et al., 2003; Durie, 1998) and an investigative approach was used to determine a new typology based on teachers' and students' dispositional characteristics (Chapter Six). In order to clarify links between the analysis and existing typologies and to ensure that new typologies were drawn as closely as possible from the data, category names were derived from pre-existing analytical frameworks, the data ("insider" terms, i.e., words commonly used by participants but not necessarily in the same way by those outside the context (Boulton \& Hammersley, 2006, p. 253)), and the researcher.

Categories were checked for "internal" and "external" homogeneity, plausibility, and credibility (Patton, 2002, p. 465) through ensuring saturation in reviewing data against the categories (i.e., no further changes to the categories). Consultation regarding the typologies was carried out with the research supervisor, both cultural advisors, and two mathematics education experts. The three typologies resulting from the study were then integrated into one holistic model (Figure 3.3, Section 3.5.1, Chapter Nine). 
Table 3.6: Main data types, analysis methods, theoretical foundations, and thesis locations of analysed data

\begin{tabular}{|c|c|c|c|}
\hline Type of data & Main analysis method/s & $\begin{array}{l}\text { Main location of } \\
\text { analysis and } \\
\text { results }\end{array}$ & $\begin{array}{c}\text { Theoretical model } \\
\text { underlying } \\
\text { analysis }\end{array}$ \\
\hline Observations & $\begin{array}{l}\text { Qualitative analysis using } \\
\text { coding and linking to pre- } \\
\text { existing typologies }\end{array}$ & Chapter Five & Durie, 1998 \\
\hline $\begin{array}{l}\text { Audio } \\
\text { recordings }\end{array}$ & Quantitative analysis & Chapter Five & Bishop et al., 2003 \\
\hline $\begin{array}{l}\text { Questionnaires } \\
\text { (Teachers) }\end{array}$ & $\begin{array}{l}\text { Quantitative analysis (carried } \\
\text { out manually) } \\
\text { Qualitative analysis using } \\
\text { coding and linking to teacher } \\
\text { interview data, pre-existing, and } \\
\text { investigative typologies }\end{array}$ & Chapter Six & \multirow{4}{*}{$\begin{array}{l}\text { Averill, Te Maro et } \\
\text { al., } 2009\end{array}$} \\
\hline $\begin{array}{l}\text { Questionnaires } \\
\text { (Students) }\end{array}$ & $\begin{array}{l}\text { Quantitative analysis (using } \\
\text { SPSS) } \\
\text { Qualitative analysis using } \\
\text { coding and linking to student } \\
\text { interview data, pre-existing, and } \\
\text { investigative typologies }\end{array}$ & $\begin{array}{l}\text { Chapters Seven } \\
\text { and Eight }\end{array}$ & \\
\hline $\begin{array}{l}\text { Interviews } \\
\text { (Teachers) }\end{array}$ & $\begin{array}{l}\text { Qualitative analysis using } \\
\text { coding and linking to teacher } \\
\text { questionnaire, pre-existing, and } \\
\text { investigative typologies }\end{array}$ & Chapter Six & \\
\hline $\begin{array}{l}\text { Interviews } \\
\text { (Students) }\end{array}$ & $\begin{array}{l}\text { Qualitative analysis using } \\
\text { coding and linking to student } \\
\text { questionnaire, pre-existing, and } \\
\text { investigative typologies }\end{array}$ & $\begin{array}{l}\text { Chapters Seven } \\
\text { and Eight }\end{array}$ & \\
\hline
\end{tabular}

Sandelowski (2003) provides examples of writing styles useful for mixed methods research approaches. Several possibilities are suitable for describing the results from studies involving concurrent qualitative and quantitative methods as used in this study (i.e., QUAL + quan ${ }^{16}$ ). Examples include using a narrative style, comparison of cases, a perspective/polyvocal style (in which shared and divergent voices are described), and a conceptual approach involving sensitising the reader to concepts, linking with theoretical frameworks and using coding families (Sandelowski, 2003). In order to best describe links between the inferences from this study to existing frameworks, to include the work of the study which triggered this research (Bishop et al., 2003), to derive concepts from existing theory, and to develop new theory, the conceptual mode of analysis and writing was selected for this thesis. Writing devices from both paradigms (e.g., visual displays, numerical values, and quotes) were integrated as appropriate.

16 The use of capitals and lower case is used to indicate that while both qualitative and that quanitative methods were used, the predominant study methods were qualitative. 
Wherever possible the original wording has been retained in quotes taken directly from the data. However, some of the quotes have been edited for reasons of coherence (as indicated by square brackets). All names are pseudonyms. The use of statistical tests for finding the degree of significance of the differences between subgroups was considered but rejected as invalid, culturally inappropriate, and unhelpful for the consideration of culturally linked data.

\subsubsection{The Teacher Care Mathematics Whare (house): A model integrating the three study typologies}

The three typologies used to analyse and describe the study data are integrated into one holistic model, 'the teacher care mathematics whare' (Figure 3.3). An adaptation of Durie's (1998) well-known model for health and well-being (discussed further in Chapter Five), the integrated model emerged during a discussion with the Māori cultural advisor regarding the study analysis results.

Durie's (1998) model is consistent with contemporary Māori thinking and was considered by the study's Māori and Pasifika cultural advisors to be an appropriate tool for analysing the study data. Similarities between the model and a Tongan perspective on human development (Koloto, 2004; Section 2.2.1) reinforce its suitability. Durie's (1998) model is used in a range of New Zealand health contexts, including the New Zealand Health and Physical Education curriculum (Ministry of Education, 1999, 2007a). It links each of four interacting dimensions of personal health and well-being to one of the four sides of a house, all necessary in combination to "ensure strength and symmetry" (Durie, 1998, p. 70). Each part of the 'teacher care mathematics whare' will be discussed in detail (Chapters FiveEight) and the parts will be brought together again in Chapter Nine.

Durie's (1998) model was chosen for the analysis of the study data from observed lessons because of its simplicity and its suitability for probing, analysing, and discussing practices of teacher care recorded within the data. Other models were also considered (e.g., Pere's (1997) te wheke, literally translated as 'the octopus'). The wheke model comprises eight components, four of which relate closely to those of the whare tapa wha. The remaining components are 'uniqueness', 'vitality', 'cultural heritage', and 'emotions'. Pere's (1997) te wheke model was rejected in part due to the number of components and difficulties categorising the observation data into some of the components (e.g., uniqueness and vitality). 
In modifying Durie's (1998) model, the whare tapa wha (literally, the four-sided house) became a 'teacher care mathematics whare', a mathematics classroom with:

- four walls, each representing one of Durie's (1998) dimensions;

- wall panels, each showing a three-strand woven design and each strand representing one of the emergent themes of the dimension;

- a whiteboard, representing the lesson elements most closely focused on mathematical thinking and learning;

- a whāriki (mat), representing interpersonal elements; and

- a roof, serving to show that the students and teacher come together in this place for mathematics learning.

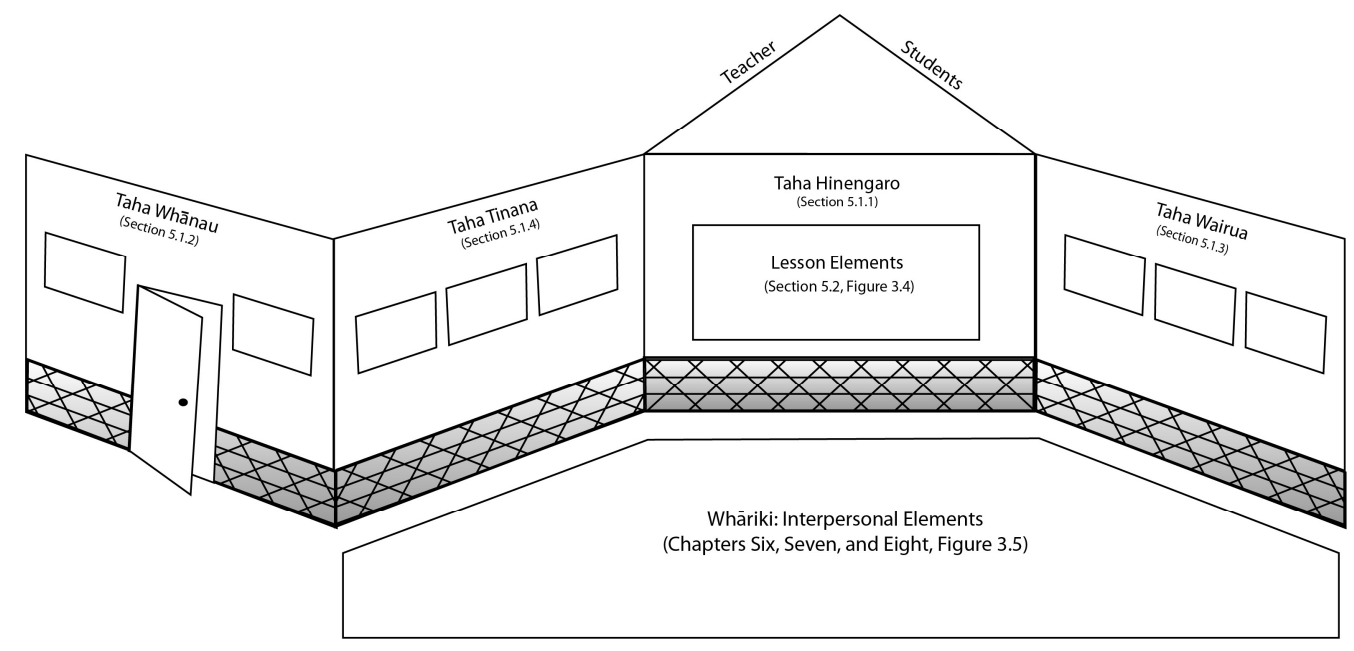

Figure 3.3: The Teacher Care Mathematics Whare

The first typology of the study is represented by the four walls of the whare. Each of the walls represents how one of the four dimensions of personal health and wellbeing in Durie's (1998) whare tapa wha are experienced in a caring mathematics classroom (Section 5.1). Analysis of the classroom observation data provided the basis for this part of the model. The taha hinengaro (Section 5.1.1) represents the purpose and main focus of the whare, the mathematical knowledge, thinking, understanding, and learning. Without mathematical learning there is no point to the whare from the teacher's, the students' or other stakeholders' perspectives (e.g., families, school, Ministry of Education). The remaining walls help represent the physical, family, and spiritual aspects of the study's observational data (Sections 5.1.2-5.1.4).

The whiteboard on the taha hinengaro (Figure 3.4) represents ways towards developing mathematical knowledge and understanding. This is illustrated by the 
whiteboard holding the second typology of the study, that of the essential mathematics lesson elements (Section 5.2), elements informed by Bishop et al.'s (2003) 'effective teaching profile'. The second typology is embedded in the first as it sits on the wall. The audio-recordings of the observed lessons and observation data were the main data sources for analysis using the second typology.

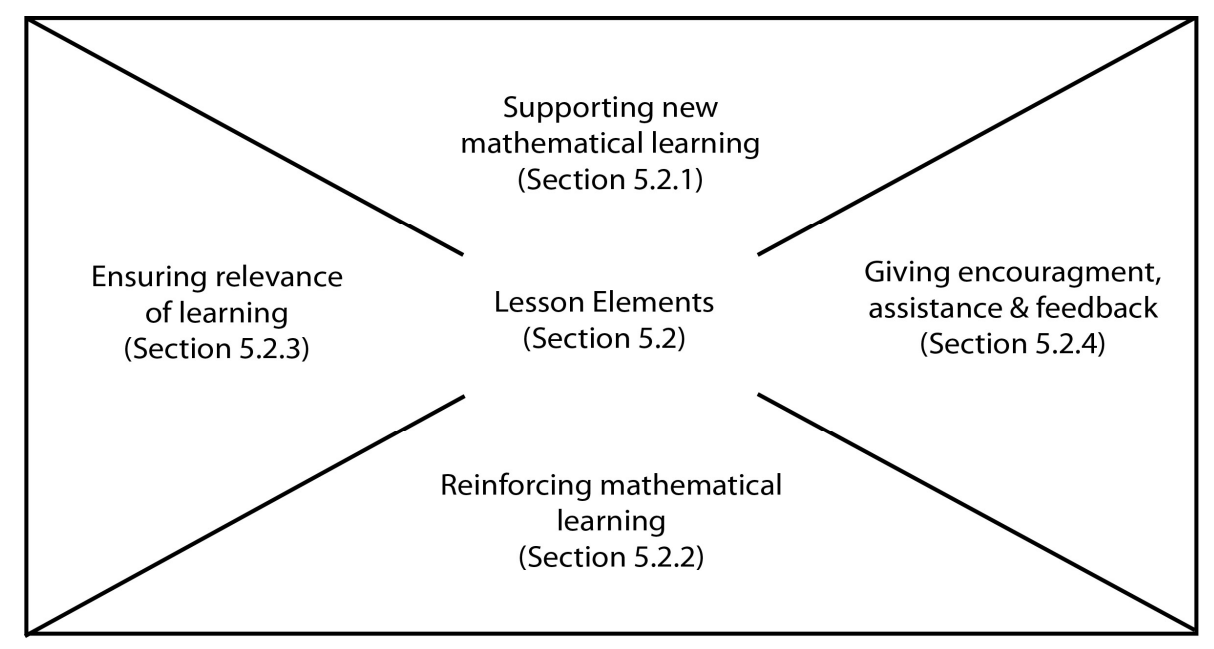

Figure 3.4: Typology for lesson elements (the whiteboard of the whare)

Analysis of the teachers' and students' questionnaire and interview data was used in conjunction with Averill, Te Maro et al.'s (2009) framework for culturally responsive teaching to generate a third typology for the study findings, represented by the whāriki (mat) which covers the classroom floor (Figure 3.5, Chapters Six-Eight). The whāriki represents the interweaving in caring mathematics classrooms of four dispositional aspects (liking, respecting, and being tolerant of each other, and reflecting one's identity) and four broad themes (knowing each other, knowing each other as learners, knowing each other's cultures, and enhancing feelings of cultural identity). 


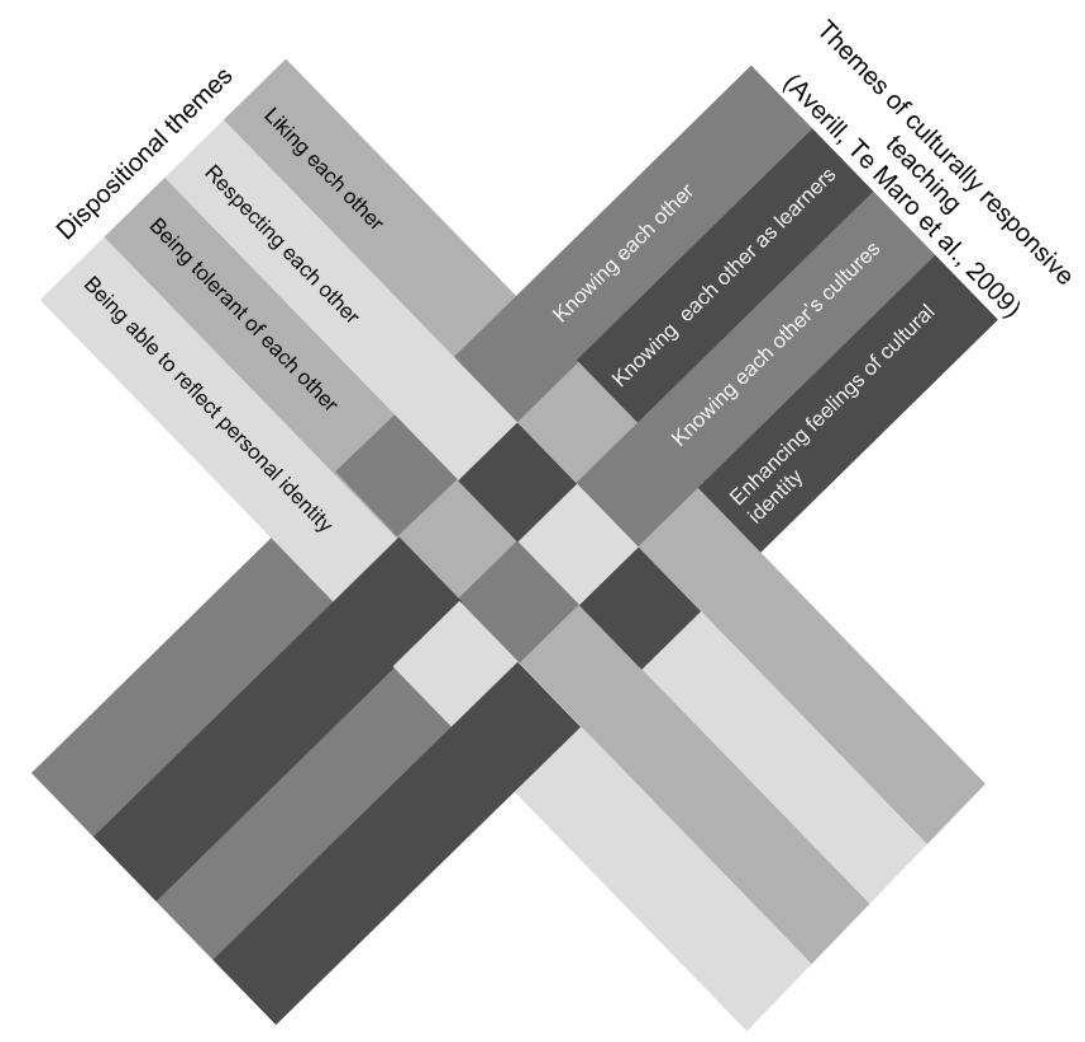

Figure 3.5: Typology of interpersonal elements (the whāriki of the whare) ${ }^{17}$

The roof of the teacher care whare represents the coming together of the teacher and students. The typologies are inter-related and all impact on the caring nature of the classroom and students' mathematics learning. Therefore each theme from the analysis should not be seen as lying only on its own two-dimensional surface; rather it should be seen three-dimensionally, as emanating from its metaphorical homebase and interacting with other themes of care within the whole whare space.

Chapter Three has introduced the study design, the study participants, the data gathering tools and their development, and how the data have been analysed. Chapter Four provides further contextual information of the study schools and participants pertinent to understanding the study data and analysis. Relevant aspects of the teacher care mathematics whare will be discussed throughout the results chapters (Chapters Five-Eight) and the model will be revisited in its entirety in Chapter Nine.

\footnotetext{
${ }^{17}$ Coincidentally, some similarities exist between the appearance of the whāriki and the organisational framework of New Zealand's Early Childhood Curriculum statement (Ministry of Education, 1996, p. 13).
} 


\section{CHAPTER FOUR}

\section{Study Schools, Teachers, and Classes}

This chapter describes the contexts within which the data were gathered and provides an overview of the study participants. Drawing from information within Education Review Office school review reports, school information, teacher interviews, and researcher perceptions, Chapter Four describes the study school environments (Section 4.1), the study teachers, their classes, and classroom contexts (Section 4.2), the study teachers' approaches to teaching mathematics (Section 4.3), and study students' ethnicities (Section 4.4). Section 4.5 summarises the contextual factors most pertinent to interpreting and analysing the study data.

\subsection{School environments}

Although the study focuses on teacher care within mathematics lessons, school environmental factors are important to consider as they can enhance or constrain teachers' expressions of care (Alton-Lee, 2003; Gay, 2000; Noddings, 1992). Examples particularly relevant to this study include the emphasis schools place on learning and on pastoral care, how inter-personal relationships are fostered, school expectations of school-wide lesson and behaviour management practices (and how teachers and students perceive these), and the emphasis schools place on care of the physical environment. This section provides a summary of the aspects of the study schools' physical, emotional, and cultural contexts most relevant to the research questions.

The researcher visited each school approximately sixty times gathering the study data. The visits included meetings and interviews with study teachers, lesson observations, and gathering questionnaire and interview data across three cycles of data gathering in each year in each school (Section 3.4). Samasama and Whero maintained tidy, well-kept school buildings and grounds. These schools felt welcoming and adults and students generally greeted and smiled at visitors. In contrast, Uliuli's physical environment looked uncared for. The gardens were poorly kept and there was always new and old rubbish around the grounds. The office entrance was sometimes crowded with boxes. Adults and students around the school tended to ignore visitors. 
Information pertinent to the study (in particular the schools' interpersonal environments, and Māori, and Pasifika dimensions) from the Education Review Office school review reports ${ }^{18}$ carried out in 2006 and 2007, immediately preceding the data collection, provides useful school contextual information. ${ }^{19}$ Individual school reports vary in the aspects and detail discussed (e.g., some reports state the number of Māori teaching staff at the school and others do not) making direct comparisons across schools difficult. However, the reports provide glimpses of the nature of each study school.

The reports for all three schools indicated the reviewers found positive and respectful relationships between teachers and students, with positive teacherstudent interactions noted at Samasama and Uliuli (Table 4.1).

\section{Table 4.1: Schools' interpersonal environments}

Note: The table entries have been drawn from the study schools' Education Review Office reports

\begin{tabular}{|l|l|}
\hline \multicolumn{1}{|c|}{ School } & \multicolumn{1}{c|}{ Interpersonal environment } \\
\hline Samasama & $\begin{array}{l}\text { positive tone in classrooms } \\
\text { warm inclusive environment } \\
\text { students are welcoming and friendly } \\
\text { generally respectful interactions } \\
\text { caring affirming approach from senior management enhances good } \\
\text { relationships within the college }\end{array}$ \\
\hline Whero & \begin{tabular}{l} 
respectful relationships between teachers and students \\
\hline Uliuli
\end{tabular} \\
$\begin{array}{l}\text { positive relationships between staff and students } \\
\text { interactions between teachers and students are generally positive and } \\
\text { supportive }\end{array}$ \\
\hline
\end{tabular}

The teachers were asked whether or not their school practised a school-wide ethic of care. Teachers at Whero reported their school did have a strong school-based ethic of care. The teachers at Samasama and Uliuli stated either that their schools did not have a school-wide approach to care or that they were not aware of one. Responses related to the role of the principal, school structures for pastoral care, personal commitment, the community, and the length of time a teacher has taught in the school.

When we look at our school our support structures are probably bigger than our academic structures. There's a huge amount of time demanded of staff in terms of pastoral work because many of the students come into the

18 The Education Review Office is a national organisation established by the government to provide external evaluations of the education for school students in all state schools and publish national reports on current educational practice. Copies of each school's review report go to the governing board of the school and the government. School reviews are also publicly available.

19 The Education Review Office reports used for the summaries identify the schools and therefore cannot be referenced in this thesis. 
classrooms with a lot of issues and the bottom line is you can't teach them unless you have some understanding of where they are coming from, not in terms of their ethnicity, but from what is going on in their homes. (WW)

I think as far as teachers caring for students and students caring for each other, personally I do believe that this comes across very strongly [at our school]... sometimes the policies might be about other things but looking out for one another and caring about one another comes through very strongly and I think that's very genuine in this school. (WK)

And so does this come from the community outside the school, or does it come from within the school, or from one another, or is it just here? (Interviewer)

I think probably about $50 \%$ comes from the school. There's a lot of flexibility and the physical structure and the beauty of the school and people can see each other quite a lot [moving around the school]. The other part is from the Pacific Island culture. It is different from the mainstream culture, there is a strong influence from that and also a lot of the families have lots of children at the school or their families are in some way connected to the school, so basically if you have been at this school for a few years the chances are you have taught a student's brother or sister. So if they don't know you personally they will know you from the connections, so that helps. If you have been here for years, the feeling will be different than just being here for a year. I care personally, but for other teachers I think some are just happy enough to get out of the classroom and on their way home. (WK)

I don't think [an ethic of care] is necessarily school wide [here]. I think the principal is a very caring person so the ethos is coming through from him. The principal uses the intercom to remind students of things like being considerate because there is a feeling that the more he repeats those kinds of expectations the more likely it is that the kids will do them. Having said that, if [student] issues are not dealt with the caring can be lost. We had an issue of a fight that affected the whole year level and I don't think it was dealt with properly and that permeated through the year level because there was a feeling of injustice. (SS)

I'm not sure at the moment, I'm not sure if it's a strong [ethic of care here]. I mean I care for the students, and it depends on what kind of students you have if they care back. Low ability students may need more attention, that's caring. (UM)

The study teachers were asked whether or not any of their school's policies helped to enhance or served to detract from teacher-student relationships (Questionnaire One, Appendix 7). Responses varied greatly between teachers, including teachers within the same school. SS and WW both held pastoral care responsibilities and may therefore have been more aware of such policies and more positive in reporting their effects. School policies and expectations reported to enhance teacher-student relationships included:

- expectations of students having respect for their teacher, themselves, and others (SS); 
- expectations of student behaviour which enhances the learning of others (SS);

- using common practices across classes (e.g., seating plans, "do now" activities for the beginning of every lesson, lesson overviews (UU)); and

- encouragement to establish effective teacher-student relationships (WW).

Other teachers (UM, ST, WK) did not recall any school policies that enhanced teacher-student relationships.

Four teachers identified school policies that detracted from teacher-student relationships. These teachers reported that large junior classes (enabling the school to offer broader option choices in the senior school) (UU, ST) and school rules (e.g., regarding lateness, chewing gum, and checking school uniform) were examples of school policies and expectations that could impact negatively on teacher-student relationships. Establishing conformity with rules was reported as starting lessons off in a negative way (SS, WK) and hindering positive teacher-student relationships and interactions. Two teachers (WW, UM) did not recall any school policies that detracted from teacher-student relationships.

It is important to consider how the school environments reflect the cultural identities of their students, particularly as the three study schools each included roughly equal numbers of Māori, Pasifika, and New Zealand European students, and a key area of the study is concerned with students' cultural identities in the classroom. The Education Review Office reports indicate that the study schools in general had systems in place for consultation with their Māori and Pasifika communities and included some Māori dimensions in their learning programmes. None of the reports commented on any Pasifika dimensions in the learning programmes. The report comments suggest that all study schools were attempting to reflect Māori and Pasifika dimensions within their school contexts but that these were not yet strongly interwoven (Tables 4.2 and 4.3). 
Table 4.2: Schools' Māori dimensions: Comments from Education Review Office reports

\begin{tabular}{|c|c|}
\hline $\begin{array}{l}\text { School, } \\
\text { aspect }\end{array}$ & Māori dimensions \\
\hline \multicolumn{2}{|l|}{ Samasama } \\
\hline $\begin{array}{l}\text { School } \\
\text { context }\end{array}$ & a bicultural dimension exists within the special character of the college \\
\hline $\begin{array}{l}\text { Learning } \\
\text { programme }\end{array}$ & $\begin{array}{l}\text { Te reo Māori (Māori language) classes available } \\
\text { opportunities for students to study te reo Māori at a local tertiary institution } \\
\text { all Year } 9 \text { students visit the local marae (traditional Māori meeting place) } \\
\text { the school monitors the academic progress of Māori students }\end{array}$ \\
\hline Consultation & $\begin{array}{l}\text { regular combined whānau and Pasifika fono (meeting) with parents and } \\
\text { students } \\
\text { established links exist with the local Māori community that enhance students' } \\
\text { identity } \\
\text { Māori representation on the Board of Trustees }\end{array}$ \\
\hline Personnel & four teachers identify as Māori ( $10 \%$ of teaching staff) \\
\hline \multicolumn{2}{|l|}{ Whero } \\
\hline $\begin{array}{l}\text { School } \\
\text { context }\end{array}$ & $\begin{array}{l}\text { follows protocols of local iwi (tribes) } \\
\text { strong kapahaka group (cultural arts group) } \\
\text { holds multicultural concerts }\end{array}$ \\
\hline $\begin{array}{l}\text { Learning } \\
\text { programme }\end{array}$ & $\begin{array}{l}\text { school-wide teacher development consistent with practices known to be } \\
\text { effective with Māori students } \\
\text { the school needs to analyse achievement data of Māori students }\end{array}$ \\
\hline Consultation & $\begin{array}{l}\text { has links with the local iwi (tribes) on the Board of Trustees } \\
\text { the school needs to consult with the Māori community over achievement of } \\
\text { Māori students }\end{array}$ \\
\hline Personnel & (no statement made) \\
\hline \multicolumn{2}{|l|}{ Uliuli } \\
\hline $\begin{array}{l}\text { School } \\
\text { context }\end{array}$ & new school wharenui (meeting house) \\
\hline $\begin{array}{l}\text { Learning } \\
\text { programme }\end{array}$ & $\begin{array}{l}\text { school-wide teacher development consistent with practices known to be } \\
\text { effective with Māori students } \\
\text { career planning opportunities aim to reflect the needs of Māori students } \\
\text { the school needs to analyse achievement data of Māori students and set } \\
\text { achievement targets for Māori students }\end{array}$ \\
\hline Consultation & the school needs to consult with the Māori community \\
\hline Personnel & (no statement made) \\
\hline
\end{tabular}


Table 4.3: Schools' Pasifika dimensions: Comments from Education Review Office

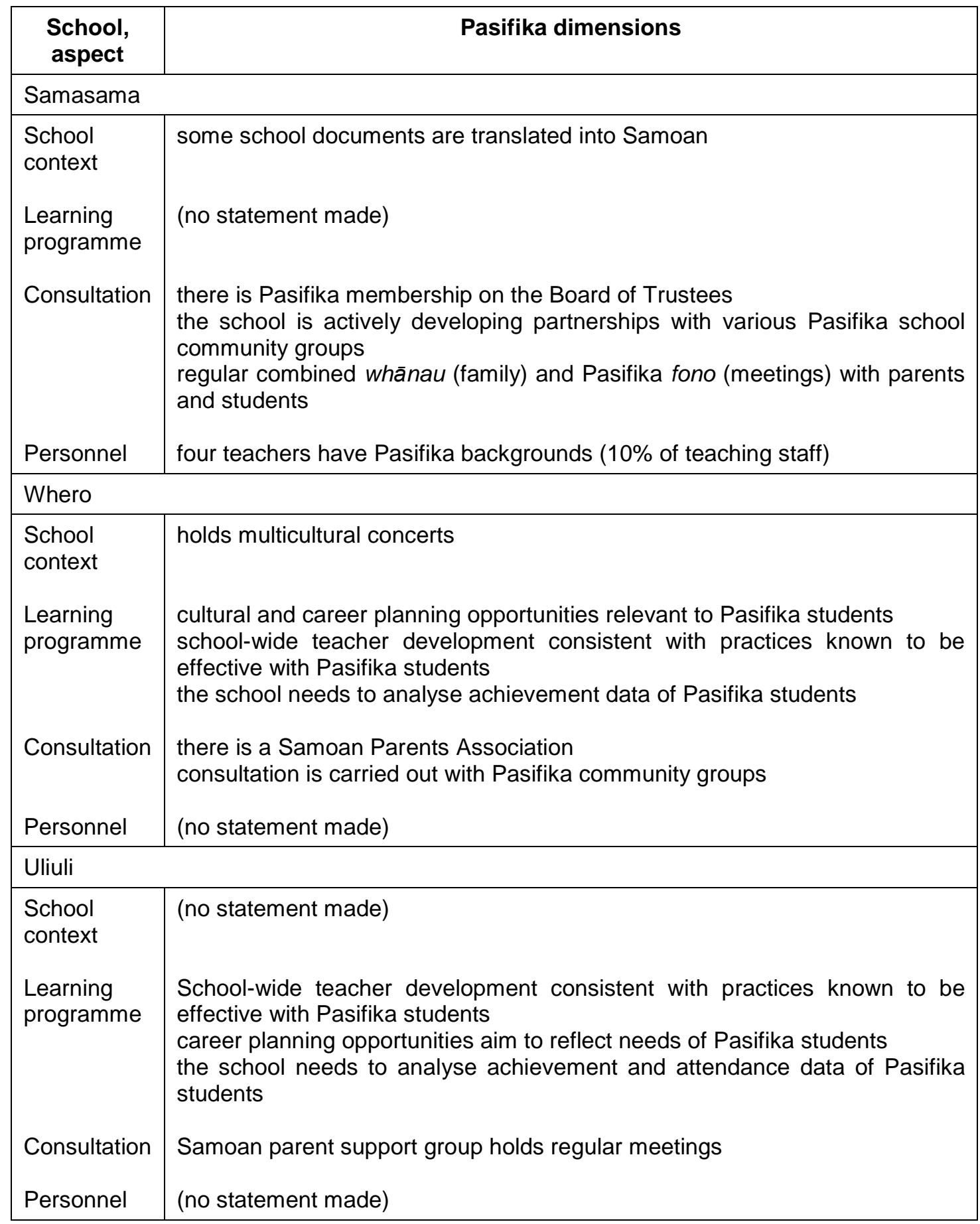

In summary, the study schools all had generally positive teacher-student interactions and each, to varying extents, reflected a school-wide ethic of care and some Māori and Pasifika dimensions. 


\subsection{Teachers, classes, and classroom environments}

This section describes for each study teacher and class: the physical and emotional classroom environment; teacher and class characteristics; interpersonal relationships; and mathematics timetable. In the discussion, teachers are grouped by school, SS and ST, then WW and WK, and finally UU and UM.

Junior classes at Samasama have four one-hour mathematics lessons a week.

SS was the Head of Department and had taught at the school for nine years. An experienced teacher of mathematics with a primary school teaching background, SS had also taught other secondary school subjects (e.g., English). In the forty-to-fifty age group, SS has New Zealand European and Māori heritage but does not speak conversational Māori. SS's classroom was colourful, pleasant, organised, and cared for. Commercial posters were well displayed. Shelves held clearly labelled storage boxes. New furniture was set out neatly in pairs and groups. The whiteboard was marked in sections, organised, and the boardwork was colourful and legible.

SS's class was the top Year 10 group, selected by overall ability. Most were together in the previous year. SS had prior pastoral care relationships with many class members as she was the dean for their year group, but had not taught these students prior to the study. SS placed importance on knowing the students. She asked all to write a letter about themselves for her within the first week of the year. SS taught the study class on Monday, Thursday, and Friday mornings, and Tuesday afternoons. The classroom atmosphere was strict, generally non-confrontational, safe, and friendly. Students were on-task and controlled. There was room for student individuality within the bounds of the lesson. SS enforced school uniform rules at the start of most lessons and interacted individually with all students several times each lesson. SS and the class appeared more comfortable with one another in Term 2 than in Term 1, consistent with a statement made in the interview carried out after the Term 2 lesson observations:

The more l've got to know them, the more l've enjoyed the class. (SS)

SS smiled and used humour more often, and students initiated conversations and humour with the teacher more readily, in Term 2 than in Term 1.

An Assistant Teacher, ST predominantly taught commerce subjects having worked in commerce prior to teaching. He had less than four years' teaching experience and 
no formal training in teaching mathematics. In his thirties, ST had taught within the school for several years. ST is a first generation New Zealander and speaks his heritage language and English at home. ST was initially anxious about being part of the study because he believed mathematics teaching not to be his teaching strength. ST's classroom was tidy and organised. There were commercially produced posters on the walls and textbooks organised tidily on shelves at the front of the room. Desks were in pairs or groups. The room was very sunny and sometimes hot. ST used the whiteboard and the overhead. The overhead could be difficult to read at times.

ST's class was a mixed ability Year 9 group. The students did not initially know one another well having come from different classes and schools. ST knew several of the students having previously taught them. ST likes to get to know students gradually through informal conversations in and out of class; however, ST spent most of each lesson at the front of the room. ST taught the study class on Monday, Tuesday, Wednesday, and Friday mornings. ST enforced a seating plan that became a cause of friction with students at the beginning of each lesson. ST did not place importance on learning students' names. In the first few days of Term 1, the classroom atmosphere was good with students paying attention and being compliant. However, this deteriorated and within the first few weeks of the year, the behaviour became noisy, rude, and generally non-compliant. ST endeavoured to maintain control and to teach, but in the researcher's opinion, his intentions were undermined mainly by his low expectations of the quantity and quality of students' work. The main changes between Terms 1 and 2 were that the negative class behaviour patterns were firmly established in Term 2. A group of the more able students was seated together in Term 2 and every lesson this group was given different work to that of the rest of the class. This group was largely on task. The teacher constantly endeavoured to establish control before explaining and giving new work to others, delaying the lesson beginning. This led to student frustration and poor behaviour.

Generally fifty minutes long, mathematics lessons were held every day for junior classes at Whero.

WW was the Head of Department and an experienced mathematics teacher, well established in the school. Having taught in the Pacific (Cook Islands, Nauru, Tokelau, and Samoa) for over ten years, WW had much experience teaching Pacific-based and New Zealand-based Pasifika students. He is New Zealand 
European and aged between fifty and sixty years old. Usually used for English and Social Studies classes, the classroom WW taught in was large, light, and well ventilated. The desks were grouped in fours and posters from subject areas other than mathematics were on the walls.

WW's class was a top Year 10 group, selected by overall ability. All but three of the class were together in the previous year. WW had taught most of the class for the year prior to the study and had prior pastoral care-based relationships with many class members, as the head of the junior school. WW prefers to get to know students slowly through exposure. WW explained his philosophy regarding knowing and accommodating students:

To teach well you've got to want to like kids no matter what you're doing. You've got to be able to put up with the rough stuff as well as the good. To get on with students well you've got to be prepared to find ways of getting around barriers (and the kids have got lots of those), and you've got to have a lot of patience with them. (WW)

WW spent extended periods of time each lesson working with students in small groups. He taught the study class on Monday, Thursday, and Friday mornings, and Tuesday and Wednesday afternoons. The classroom atmosphere was comfortable but students often worked slowly or were off-task. WW was generally nonconfrontational and placed emphasis on students being self-motivated and taking responsibility for their learning. Students could be themselves during mathematics lessons and were able to move around the room, eat, drink, and play music as long as WW considered they were getting their work done. WW occasionally raised issues of conformity with school rules such as uniform requirements. There was little change in teacher-student rapport noticed between Terms 1 and 2. However, in Term 2, the three students new to the class in Term 1 were more positive about their relationship with the teacher and about their maths work than they had been at the start of the year.

WK was the Assistant Head of Department with over thirty years' experience teaching mathematics. Originally with a primary school teaching background, WK was well established in the school. He is New Zealand European but grew up in a colonial community in which one's race determined one's life chances and expectations. WK was aged between fifty and sixty. WK's classroom was tidy with mathematical, colourful, and engaging classroom displays created by his students and him. WK's boardwork was immaculate. He considered the classroom his and the students guests. 
WK's group was a top Year 10 class, selected by overall ability. WK knew all but a few students well at the start of the year, having taught the class for the previous year. Respectful and caring teacher-student relationships were in place. WK clearly enjoyed the company of the students, was relaxed, and had a jovial but firm manner. WK taught the study class on Monday, Tuesday, and Friday mornings, and Wednesday and Thursday afternoons. WK made mathematics learning the priority in every lesson and had high expectations of the students. He shared these through his teaching style and by telling the students of his expectations. There was a strict but fair classroom atmosphere. WK constantly moved around the room helping students and checking their progress. WK enjoyed teaching this class:

I think every lesson with them is great, I just sort of enjoy teaching them; I mean some days are worse than others but most days I look forward to teaching them. (WK)

There were no major changes in teacher-student relationships or classroom atmosphere apparent between Terms 1 and 2 .

Two differences between Whero and the other study schools, both aimed at improving achievement, may have helped enhance teacher-student relationships:

- a new junior school structure had been implemented at the beginning of Phase One data collection:

...we felt that by changing the structures we could get the kids more incentive to achieve. In changing structures I actually think the kids might develop better relationships with their teachers as well (WW); and

- many junior mathematics classes kept the same mathematics teacher for two years.

Uliuli used a six-day timetable. Year 10 mathematics was taught on four consecutive days, thus a long break between subsequent mathematics lessons occurred when the two days with no mathematics were combined with the weekend. This effect was magnified on public holiday weekends and when the last mathematics lesson before the four-day break was the final lesson of the day, a challenging time of the school day for mathematics learning.

UU was the Head of Department and an experienced teacher of mathematics. In the fifty-to-sixty age group, UU was originally trained as a primary school teacher. She is New Zealand European, had taught at the study school for one year prior to the study, had previously taught at schools similar to the study school, and had worked in tertiary mathematics education. UU's classroom was fairly bare. Well ventilated, the 
classroom had large tables each with four chairs. UU's class was a mixed ability Year 10 group. Drawn from many Year 9 classes from the previous school year, the class did not initially know one another well. UU had taught several of the students in the previous year but had no pre-existing relationships with the rest of the class. She likes to learn students' names quickly and to know more about the students over time.

UU had two morning and two afternoon lessons with this class in each six-day cycle. Lessons varied from having a pleasant and productive atmosphere to being loud and off task. Although UU expected compliance, her approach was generally nonconfrontational with, at times, low expectations of the quality and quantity of student learning. UU reinforced school rules and procedures frequently, for example by requiring late passes when students were late. UU made the students line up outside the classroom before each lesson and often made them wait until all were quiet. Changes were noticed in teacher-student relationships between Terms 1 and 2, for example, the students challenged the teacher less frequently during the Term 2 lesson observations than in Term 1. UU appeared much more comfortable with the class in Term 2, was smiling more, and making more use of valid praise than in Term 1; however, many students worked very slowly or were off task for long spells of many lessons.

UM was an Assistant Teacher. He was in his first few years of teaching and had been at the school for all of this time. Mathematics was his preferred subject area and he was new to teaching top band junior classes. Aged between thirty and forty, UM is a first generation New Zealander with English his second language. UM's classroom was small for the size of the class and rather barren with little or no student work on the walls. His desk was near the front and it and the surrounding shelves were untidy. UM used a loudspeaker system to amplify his voice. The desks were in pairs or groups.

UM's class was a top Year 10 group, selected by overall ability. UM had taught several of the study students in the year prior to the study and had a friendly, firm manner. He used humour to help manage student behaviour and got to know the students' names quickly. He liked to get to know the students gradually. UM had two morning and two afternoon lessons with this class in each six-day week. The atmosphere was pleasant, generally non-confrontational, and focused on mathematics learning. UM was always available to help students and, when not using teacher exposition, constantly moved around the classroom. The two main changes noticed in UM's classroom between Terms 1 and 2 were that later in the year he made more use of students' names and there was an increase in studentinitiated interactions. 


\subsection{Study teachers' teaching of mathematics}

The study teachers' approaches to teaching mathematics were varied; however, each individual study teacher's lessons followed a fairly consistent format (Table 4.4). Most presented mathematics as a set of skills to be learned and mastered, WW the exception, placing much emphasis on problem solving and seeking patterns. Whole class teaching and one-to-one assistance were the predominant teacher activities across classes. SS's, WK's, and UM's students were consistently engaged with mathematics learning, WW's class, moderately engaged on average, and the engagement of ST's and UU's classes varied.

Table 4.4: Study teachers' lessons (mathematics teaching and learning)

\begin{tabular}{|c|c|c|c|c|}
\hline Teacher & $\begin{array}{l}\text { What constitutes } \\
\text { mathematics in this } \\
\text { teacher's classroom }\end{array}$ & Lesson structure & $\begin{array}{l}\text { Main } \\
\text { lesson } \\
\text { activity } \\
\text { (teacher) }\end{array}$ & $\begin{array}{l}\text { Main } \\
\text { lesson } \\
\text { activity } \\
\text { (students) }\end{array}$ \\
\hline SS & $\begin{array}{l}\text { Rule-bound approach, } \\
\text { games, notes, } \\
\text { explanations, and } \\
\text { examples, teaches for } \\
\text { understanding }\end{array}$ & $\begin{array}{l}\text { Homework check } \\
\text { Review/game } \\
\text { Exposition } \\
\text { Textbook work } \\
\text { Marking/review } \\
\text { Setting homework }\end{array}$ & $\begin{array}{l}\text { Teacher- } \\
\text { whole class } \\
\text { teaching }\end{array}$ & $\begin{array}{l}\text { Engaged in } \\
\text { mathematics } \\
\text { learning }\end{array}$ \\
\hline ST & $\begin{array}{l}\text { Rule-bound approach, } \\
\text { notes, blackboard, and } \\
\text { textbook questions }\end{array}$ & $\begin{array}{l}\text { Starter activity on overhead } \\
\text { Exposition } \\
\text { Whole class marking } \\
\text { Textbook work } \\
\text { 1-to-1 assistance }\end{array}$ & $\begin{array}{l}\text { Teacher- } \\
\text { whole class } \\
\text { interaction }\end{array}$ & Talking \\
\hline WW & $\begin{array}{l}\text { Problem solving, } \\
\text { pattern seeking, } \\
\text { worksheets written by } \\
\text { teacher, teaches for } \\
\text { understanding }\end{array}$ & $\begin{array}{l}\text { Sets new work } \\
1 \text {-to-1 and 1-to-group } \\
\text { assistance } \\
\text { (prolonged assistance to } \\
\text { each) } \\
\text { At times asks for and gets } \\
\text { attention for explanations }\end{array}$ & $\begin{array}{l}\text { Roving and } \\
1 \text {-to-1 help }\end{array}$ & Talking \\
\hline WK & $\begin{array}{l}\text { Rule-bound approach, } \\
\text { blackboard and } \\
\text { textbook questions, } \\
\text { some relating of } \\
\text { examples to real life, } \\
\text { teaches for } \\
\text { understanding }\end{array}$ & $\begin{array}{l}\text { Many review questions } \\
\text { Homework check } \\
\text { Exposition } \\
\text { Textbook work } \\
\text { 1-to-1 helping } \\
\text { Setting homework }\end{array}$ & $\begin{array}{l}\text { Teacher- } \\
\text { whole class } \\
\text { teaching, } \\
\text { roving and } \\
1 \text {-to-1 help }\end{array}$ & $\begin{array}{l}\text { Engaged in } \\
\text { mathematics } \\
\text { learning }\end{array}$ \\
\hline UU & $\begin{array}{l}\text { Rule-bound approach, } \\
\text { review and worksheet } \\
\text { questions }\end{array}$ & $\begin{array}{l}\text { Lines class up outside } \\
\text { classroom } \\
\text { Review questions } \\
\text { Lesson organiser on board } \\
\text { Brief exposition } \\
\text { Worksheets }\end{array}$ & $\begin{array}{l}\text { Roving and } \\
1 \text {-to-1 help }\end{array}$ & Talking \\
\hline UM & $\begin{array}{l}\text { Pattern seeking, } \\
\text { practice of examples, } \\
\text { teaches for } \\
\text { understanding }\end{array}$ & $\begin{array}{l}\text { Review questions } \\
\text { Exposition } \\
\text { Textbook work } \\
\text { 1-to-1 helping }\end{array}$ & $\begin{array}{l}\text { Teacher- } \\
\text { whole class } \\
\text { teaching, } \\
\text { roving and } \\
1 \text {-to-1 help }\end{array}$ & $\begin{array}{l}\text { Engaged in } \\
\text { mathematics } \\
\text { learning }\end{array}$ \\
\hline
\end{tabular}


Teachers' views of learning mathematics were revealed both through their teaching and in their interview responses regarding how teaching mathematics differed from teaching other subjects (Interview Three, Appendix 11). Responses indicated that the teachers viewed mathematics as more structured than other subjects, right or wrong, more dependent on prior knowledge, and at times, less interesting for students or less clearly related to students' lives and needs:

[Maths] is much more structured. It's much more black and white. So kids do it, they check that it's right. As a teacher it's easier to know what's right or wrong. (SS)

It uses paper and pen more than other subjects and doing games, so it's not huge amounts of practical stuff always. A lot of people have the idea that maths is right or wrong. I suppose it is a little bit more clear cut than some of the other subjects but there's still different ways of doing things - maybe most people end up with the same kind of result but there are different paths to it. (UU)

While some of the subjects have continuity it's not quite so paramount as it is in mathematics because mathematics is fundamentally building one block on top of another. You miss one little block and you can fail the whole concept because you just don't understand. For example, you may understand what $10 \%$ of 20 means, but if you don't understand how to change $10 \%$ into a decimal then you're going to have problems with it and unless someone reteaches that to you, you can't do the subsequent lessons. (WK)

Kids are less inherently interested in maths than in other subjects like science. (WW)

Then you've got the added complication in mathematics that they've got to learn a whole new language really because maths is another language. So they've got to learn the language of maths and then they've got to try and see the relevance of it to themselves. And seeing the relevance of this you know we start saying 'find $10 \%$ of 20 '... well why? Why do I want to find $10 \%$ of 20 ? Until you can see the relevance of maths it's just an abstract subject to some extent. It's like doing bearings with my Year 9s. Why do they want to know 3 digit bearings? They're not going to be navigators. They're just sitting in a classroom doing these $3^{\circ}$ North, $35^{\circ}$ West... and so it goes on. What's the relevance? I think unfortunately there's a lot of maths that is not relevant to what the kids need to know, but at the same time I think they've got to do it because it increases their thought processes, their ability to handle different situations. (WK)

Study teachers' approaches to the management of student learning varied (Table 4.5). Those with higher expectations of student work output constantly reinforced these expectations, and tended to include a wider range of activity styles in each lesson (e.g., starter, homework check, new learning, game, marking of new work, 
calculator activity, discussion, etc). The less engaged classes were the mixed ability classes (ST, UU), and those of the teachers who left the responsibility of being engaged to the students (WW), who did not provide sufficiently challenging work (ST, UU), and who did not appear to expect full engagement (ST, UU).

Table 4.5: Study teachers' lessons (teachers' expectations and use of praise)

\begin{tabular}{|c|c|c|c|}
\hline Teacher & $\begin{array}{l}\text { Apparent responsibility } \\
\text { for learning in this } \\
\text { classroom }\end{array}$ & $\begin{array}{l}\text { Apparent teacher } \\
\text { expectations of } \\
\text { students' work }\end{array}$ & Teacher's use of praise \\
\hline SS & $\begin{array}{l}\text { Controls all aspects of } \\
\text { lesson, very structured } \\
\text { approach. }\end{array}$ & High and overt. & $\begin{array}{l}\text { Publicly and privately stated. } \\
\text { Also uses public } \\
\text { admonishment. }\end{array}$ \\
\hline ST & $\begin{array}{l}\text { The students control the } \\
\text { lesson. }\end{array}$ & $\begin{array}{l}\text { Low } \\
\text { Sets easy work. }\end{array}$ & $\begin{array}{l}\text { Little or no use of praise. } \\
\text { Public admonishment. }\end{array}$ \\
\hline WW & $\begin{array}{l}\text { Encourages students to } \\
\text { take responsibility for } \\
\text { their work level and } \\
\text { achievement. Teacher } \\
\text { sets work but does not } \\
\text { insist students work hard. }\end{array}$ & $\begin{array}{l}\text { Students state WW } \\
\text { has high } \\
\text { expectations of } \\
\text { them. Some } \\
\text { evidence of this in } \\
\text { lesson } \\
\text { observations. }\end{array}$ & $\begin{array}{l}\text { Privately stated and stated in } \\
\text { general sense regarding the } \\
\text { work of the whole class } \\
\text { School points system rewards } \\
\text { good work. }\end{array}$ \\
\hline WK & $\begin{array}{l}\text { Controls all aspects of } \\
\text { lesson, very structured } \\
\text { approach. }\end{array}$ & High and overt. & $\begin{array}{l}\text { Publicly and privately stated. } \\
\text { Uses praise frequently. }\end{array}$ \\
\hline UU & $\begin{array}{l}\text { Sets work but does not } \\
\text { insist students work hard. }\end{array}$ & $\begin{array}{l}\text { Low } \\
\text { Not overt. } \\
\text { Sets easy work to } \\
\text { help students feel } \\
\text { success and build } \\
\text { student confidence. }\end{array}$ & $\begin{array}{l}\text { Public and privately stated. } \\
\text { Praise is sometimes invalid. }\end{array}$ \\
\hline UM & $\begin{array}{l}\text { Students' responsibility } \\
\text { supported with } \\
\text { encouragement to work } \\
\text { well and 1-to-1 } \\
\text { assistance. }\end{array}$ & Moderate to high. & Publicly and privately stated. \\
\hline
\end{tabular}

Teachers SS, WW, WK, and UM helped students to understand the intended lesson aims and teaching strategies by explaining and justifying their teaching decisions. Teachers ST and UU often presented the lesson's work without explaining the rationale for the chosen activities and expected compliance.

Given a list of possible influences on their teaching of the study classes, teachers reported that most influential was their experiences teaching these classes (Questionnaire One, Appendix 7), exemplified well by the following statement:

In the class for me it's twenty-three different interactions. You can't treat them all the same way individually and when they're in groups you can't treat all the same as you would individually either, in that each group functions differently. So for me it's shutting up and watching in the first instance. (WW) 
'Teaching similar classes' and 'discussions with students' were also seen to be very important with all teachers agreeing or strongly agreeing that these influenced their teaching of these classes. Other influential factors included 'discussions with colleagues' (UM, WW, WK, and SS), 'professional reading' (UM, SS, and UU) and 'professional development' (WW and SS). None of the teachers reported any further influences on their teaching of the study classes. These results indicate the great value placed by study teachers on experiential learning for improving their teaching of specific class groups. This supports the view that teaching groups of individuals is enhanced by knowing the individuals and how they individually and collectively interact with their classmates and react to teacher input. Such knowledge can only be gained through experience teaching the group and associated teacher reflection.

\subsection{Students' ethnicities: Student data vs school data}

The Ministry of Education quotes Statistics New Zealand's (Ministry of Education, 2005, 2006a) definition of 'ethnicity' as:

the ethnic group or groups that people identify with or feel they belong to. Ethnicity is a measure of cultural affiliation, as opposed to race, ancestry, nationality or citizenship. Thus, ethnicity is self-perceived and people can affiliate with more than one ethnic group. (2006a, p. 18)

The enrolment forms for students enrolling in New Zealand schools allow identification of up to three ethnic groups as selected by students or their parents/guardians. However, the Ministry of Education requires that New Zealand students be reported in only one ethnic group on the school roll characteristics information collected from schools. Each student's reported ethnicity is determined using the Roll Return Guidelines (Ministry of Education, 2005, 2006a). Where more than one ethnic group has been identified, the ethnicity recorded for the Ministry of Education is determined using the system of priority recording used by Statistics New Zealand in the 2001 census (Ministry of Education, 2005, 2006a). The results of this process include that:

- the ethnicity of all students is recorded as one of five ethnic groups: Māori; Pasifika; ${ }^{20}$ Asian; Other; and New Zealand European;

- all students who have identified Māori alongside an/other ethnicity/ies are recorded as Māori (despite the relative depth of personal identification with their heritage ethnicities); and similarly

20 'Pacific Peoples' was the term used in 2007 (Ministry of Education, 2006a). In 2006, the corresponding term was Pasifika (Ministry of Education, 2005). 
- all students who have identified as Pasifika students alongside an/other ethnicity/ies are recorded as Pasifika (unless Māori is one of their other noted ethnicities).

Tables 3.4 and 3.5 respectively show the Ministry of Education ethnicity data of the study schools and classes.

The study data highlight the limitations of the accuracy of the Ministry of Education data as the ethnicity data provided by the study schools and the data provided by study students varied greatly (Figures 4.1 and 4.2). Study students were asked (Questionnaire One) to indicate all ethnicities that applied to them: almost half (42\%) identified with more than one ethnicity, with $37 \%$ and $5 \%$ indicating two and three ethnicities respectively. Overall five combinations of ethnicities were selected: Māori and European (22\% students), Pasifika and European (9\%), Māori and Pasifika (5\%), Māori, Pasifika and European (4\%), and Pasifika, European, and Other (1\%).

The comparison is shown using the two types of ethnicity results (Ministry of Education data and self-reported data) for the students who indicated their ethnicity in Questionnaire One, $70 \%$ of the total student sample. It would be reasonable to assume that this is a representative group from the complete student sample and many New Zealand students in multicultural schools also claim more than one ethnicity.

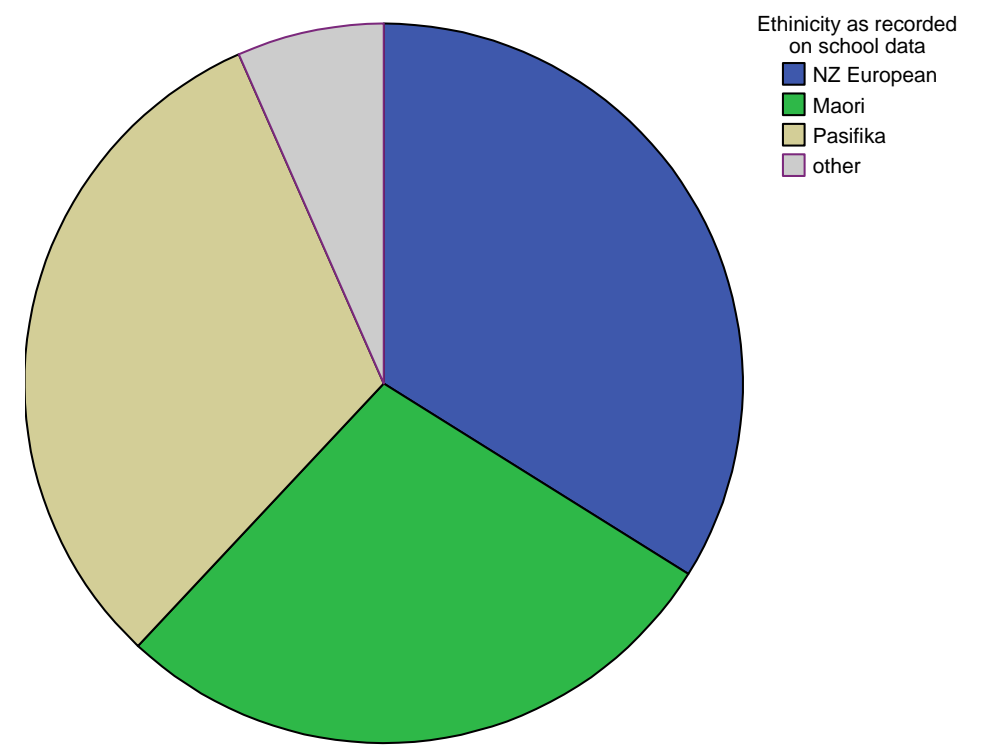

Figure 4.1: Students' ethnicity as recorded in Ministry of Education data Note: Students who completed Questionnaire One 


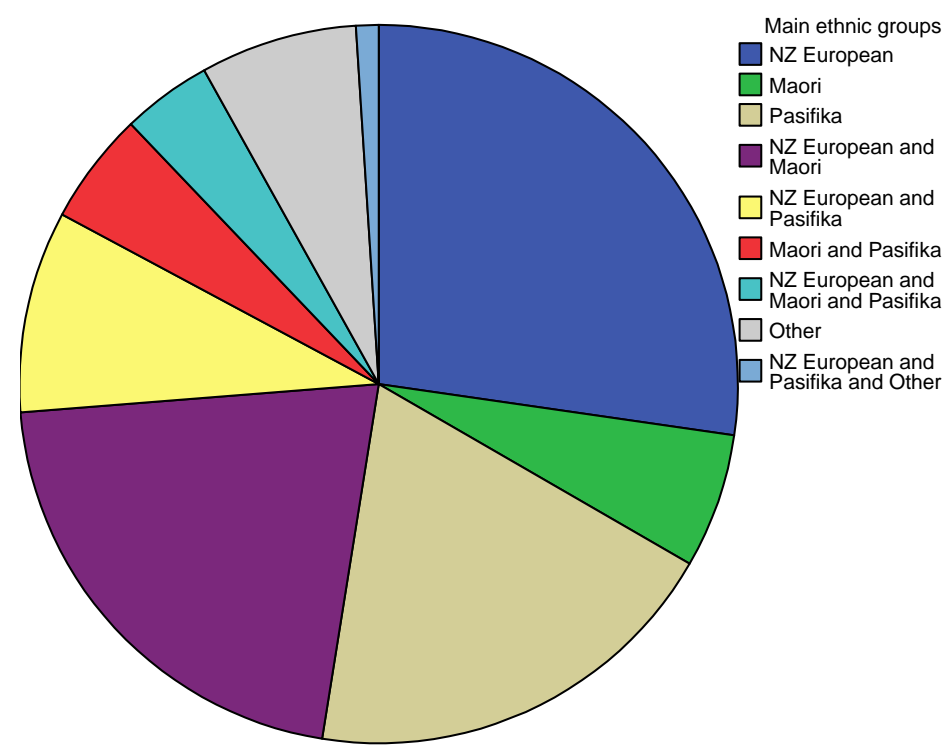

Figure 4.2: Students' self-reported ethnicity

Note: Students who completed Questionnaire One

Students who identified themselves as Māori (in Questionnaire One) were not asked to identify their heritage iwi and none did so. Students who indicated Pasifika heritage were asked to state their heritage country and all did so as did some New Zealand European students, illustrating further limitations to the accuracy of how well the Ministry of Education ethnicity data represent students' heritage claims. The differences between school ethnicity data and data provided by students indicate that although a convenient way to explore differences between New Zealand students' ethnic groups nationally, research and publications using the Ministry of Education's ethnicity data (as recorded and provided by schools) should be interpreted with some caution.

Because of the comparatively small study sample size, the diverse combinations of students' self-reported ethnicities, and the incomplete set of student-reported ethnicity data, ${ }^{21}$ many study results (including most student quotes) included in the results chapters do not highlight students' ethnicities. Where apparent differences emerged between the data from students of different ethnic groups, students' ethnicity is reported in one of two ways depending on the most suitable type of data for analysis or comparison: students' self-reported ethnicities; or school-recorded ethnicity data:

21 Only 96 of the 136 student participants completed Questionniare One in which students reported their ethnicity. Furthermore, students' ethnicities were not able to be determined for many of the student interviews because in an attempt to ensure students felt confident their comments would be treated confidentially, student names were not always recorded. A further complicating factor was that many student interviews were conducted with students in pairs. 
- $M a ̄ o r i{ }^{*}\left(M^{*}\right)$, Pasifika* $\left(P^{\star}\right)$, New Zealand European* $\left(E^{\star}\right)$, include all students who identified each as part of their heritage ${ }^{22}$ (Chapters Seven and Eight). ${ }^{23}$ The numbers of students in each group were: 35 Māori*; 34 Pasifika*; and 53 New Zealand European*; and

- Māori (M), Pasifika (P), New Zealand European (E) indicate use of the school ethnicity data (Chapters Seven and Eight). The numbers in each group were: 46 Māori; 52 Pasifika; and 52 New Zealand European.

Student totals include all students (Māori, Pasifika, New Zealand European and all other ethnicities).

The school's ethnicity data were used for most of the analysis because:

- school ethnicity data were available for a larger proportion of students (Questionnaire Three provided the main data source for Chapter Eight but Questionnaire One was completed by only $70 \%$ of those completing Questionnaire Three);

- these data allow closer comparisons with related data in Ministry of Education and other New Zealand publications; and

- the students' self-reported ethnicity data were comparatively complex.

\subsection{Discussion}

Clearly, school environments affect the general sense of care in the school; however, the caring nature of the school environment can be over-ridden within individual classrooms in which the levels of teacher care for students and their learning are the predominant influences on classroom atmosphere. All study teachers' practice included caring and less caring elements. Some teachers included more caring elements than others. The study data indicated that the teachers who ensured students were kept on task with work set at appropriate levels, varied learning activities, attended to details, and placed importance on knowing their students as individuals appeared best placed to establish and maintain caring teacher-student relationships (Chapters Five-Nine). SS's and WK's classrooms were those in which most students were consistently on task and in which the greatest levels of teacher care were apparent, followed by WW's and UM's classrooms, and lastly those of UU and $\mathrm{ST}^{24}$.

22 Therefore many students are counted in more than one group.

23 This method was discussed with and endorsed by the cultural advisors.

24 It should be noted that all study teachers showed care for their students. None were uncaring. 


\section{CHAPTER FIVE}

\section{The Mathematics Lessons}

This chapter presents the researcher's perceptions of teacher care as observed in the study classrooms in light of the literature (Chapter Two) and consultation with cultural advisors. The findings from analysis of the data gathered from the lesson observations and lesson audio-recordings are described using two typologies to organise the discussion. The first is drawn from Durie's (1998) whare tapa wha model for health and well-being. The second, drawn from elements of Bishop et al.'s (2003) "effective teaching profile" (pp. 97-118) and themes from the study results, is embedded within Durie's (1998) model (Section 3.5.1, Figure 5.1, Table 5.1).

In order to provide a clear and detailed discussion of teacher care in mathematics lessons, this chapter begins by using the first typology to describe a holistic view of teacher care in the mathematics classroom and to compare the practices used in comparatively care-rich and care-poor classroom lessons (Section 5.1). The discussion then narrows its focus to examine the elements of teacher care most closely related to mathematics teaching and learning using the second typology to highlight specific caring mathematics teaching practices (Section 5.2). Key ideas from both of these sections are then drawn together in a description and analysis of the lesson that best exemplified the three aspects of care explored in this study (Section 5.3). Section 5.4 introduces a brief discussion of the results from the lesson observations relating to teacher care for students as culturally located individuals, a theme which will be further explored in Chapters Six and Eight. Finally key themes from the chapter are discussed in Section 5.5.

A three-stage consultation process was used to ensure that culturally related classroom observation data were appropriately interpreted and incorporated. Cultural advice was sought to inform researcher decision-making with respect to a possible framework for analysing observational data (previously discussed in Chapter Three), the subsequent data analysis, and the final chapter content.

Analysis of data from student questionnaires, student interviews, and lesson observations indicated that of the six teachers observed, teachers SS and WK exhibited the greatest teacher care overall (Section 4.5). In order to understand teacher care more clearly, the analysis presented in this chapter privileges the practices of the most 
caring teachers and examines the main differences between these and the least caring teacher practices.

\subsection{Comparison of the two most and two least caring classrooms}

The observational data were analysed using Durie's (1998) whare tapa wha (literally translated as 'the four-sided house') model of health and well-being modified to fit the mathematics classroom (Section 3.5.1, Figure 5.1, Table 5.1). The four dimensions of the whare are taha hinengaro, taha whānau, taha wairua, and taha tinana (Sections 5.1.1-5.1.4, Table 5.1). Taha hinengaro represents people's mental, psychological, and emotional well-being and includes the expression of thoughts, feelings, and communication. Particularly relevant to this study is the importance of body language as a means of communication for Māori and Pasifika people:

Māori may be more impressed by the unspoken signals conveyed through subtle gesture, eye movement, or bland expression, and in some situations regard words as superfluous, even demeaning. Emotional communication can assume an importance which is as meaningful as an exchange of words and valued just as much. (Durie, 1998, p. 72)

Taha whānau refers to the dimension of our lives relating to our extended family, our "capacity to belong, to care, and to share" (Durie, 1998, p. 70). Physical well-being, growth, and development are encapsulated in taha tinana, and taha wairua represents the spiritual elements of our well-being including "the capacity for faith and wider communion" (p. 70). Balance across the four dimensions is important, with taha wairua (the spiritual side) generally being seen by Māori as the most essential of the four for good health (p. 71).

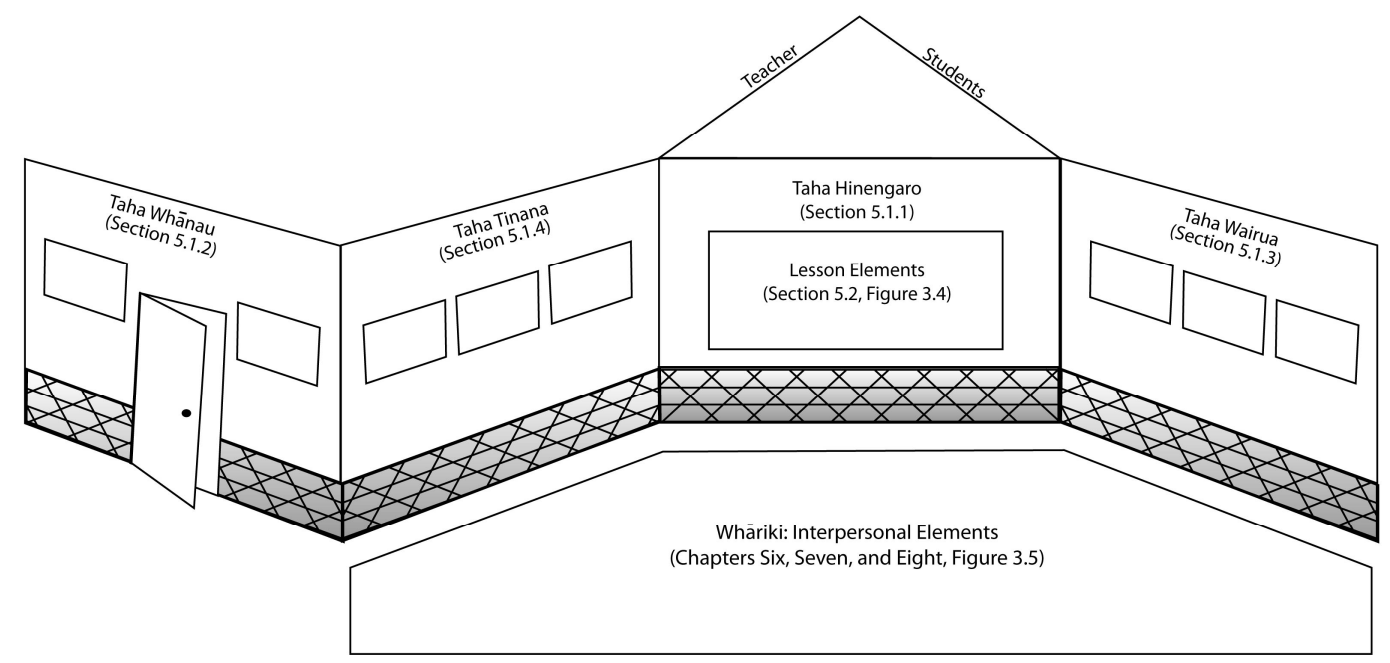

Figure 5.1: The Teacher Care Mathematics Whare 
Table 5.1: Viewing the observation data using Durie's (1998) whare tapa wha model for health and well-being

\begin{tabular}{|c|c|c|}
\hline Dimensions & $\begin{array}{l}\text { Characteristics of caring } \\
\text { mathematics classrooms }\end{array}$ & Teacher qualities and behaviours \\
\hline \multirow{3}{*}{$\begin{array}{l}\text { Taha } \\
\text { hinengaro } \\
\text { (relating to the } \\
\text { expression of } \\
\text { thoughts and } \\
\text { feelings) } \\
\text { (Sections } \\
5.1 .1 \text { and 5.2) }\end{array}$} & $\begin{array}{l}\text { Learning environment: } \\
\text { - teachers create a safe and } \\
\text { purposeful learning } \\
\text { environment (Section 5.1.1) }\end{array}$ & $\begin{array}{l}\text { Teachers are consistent, firm, and explicit } \\
\text { about their practice and expectations. } \\
\text { Teachers interact personably and } \\
\text { respectfully and capitalise on student } \\
\text { reactions and responses. }\end{array}$ \\
\hline & $\begin{array}{l}\text { - teachers create a fun and } \\
\text { engaging learning } \\
\text { environment (Section 5.1.1) }\end{array}$ & $\begin{array}{l}\text { Teachers share decision-making, vary } \\
\text { lesson activities, incorporate fun, set high } \\
\text { expectations, and utilise humour. }\end{array}$ \\
\hline & $\begin{array}{l}\text { - teachers create a learning } \\
\text { environment focused on } \\
\text { mathematical learning } \\
\text { (Section 5.2) }\end{array}$ & $\begin{array}{l}\text { Teachers attend to emotional, physical, and } \\
\text { psychological mathematical learning needs. }\end{array}$ \\
\hline \multirow[t]{3}{*}{$\begin{array}{l}\text { Taha whānau } \\
\text { (relating to the } \\
\text { extended } \\
\text { family and } \\
\text { relationships) } \\
\text { (Section } 5.1 .2 \text { ) }\end{array}$} & $\begin{array}{l}\text { Community: } \\
\text { - teachers nurture a sense } \\
\text { of community }\end{array}$ & $\begin{array}{l}\text { Teachers are inclusive, dependable, and } \\
\text { capitalise on shared endeavours. } \\
\text { Teachers value personal identities, } \\
\text { participate in activities, incorporate pair and } \\
\text { group work, and encourage students' } \\
\text { support of one another. }\end{array}$ \\
\hline & $\begin{array}{l}\text { - teachers nurture a sense } \\
\text { of personal responsibility }\end{array}$ & $\begin{array}{l}\text { Teachers discuss high learning-related and } \\
\text { behavioural expectations and choices and } \\
\text { consequences. } \\
\text { Teachers encourage shared responsibility } \\
\text { for learning and behaviour in and outside } \\
\text { the classroom. }\end{array}$ \\
\hline & $\begin{array}{l}\text { - teachers focus on } \\
\text { mathematical learning }\end{array}$ & $\begin{array}{l}\text { Teachers acknowledge prior learning, } \\
\text { support and reinforce new learning, attend } \\
\text { to relevance of content, encourage, assist, } \\
\text { and give feedback. }\end{array}$ \\
\hline \multirow{3}{*}{$\begin{array}{l}\text { Taha wairua } \\
\text { (relating to the } \\
\text { spiritual } \\
\text { elements of } \\
\text { well-being) } \\
\text { (Section } 5.1 .3 \text { ) }\end{array}$} & $\begin{array}{l}\text { Personal identity: } \\
\text { - teachers model and expect } \\
\text { respectful interactions }\end{array}$ & $\begin{array}{l}\text { Teachers respect students and their work, } \\
\text { facilitate help seeking, and provide timely } \\
\text { and specific feedback. }\end{array}$ \\
\hline & $\begin{array}{l}\text { - teachers prioritise one-to- } \\
\text { one interactions }\end{array}$ & $\begin{array}{l}\text { Teachers privilege one-to-one teacher- } \\
\text { student interactions in order to praise, } \\
\text { acknowledge, and encourage. }\end{array}$ \\
\hline & $\begin{array}{l}\text { - teachers focus on } \\
\text { mathematical learning }\end{array}$ & $\begin{array}{l}\text { Teachers facilitate and encourage students' } \\
\text { sense of personal mathematical identity. }\end{array}$ \\
\hline \multirow{3}{*}{$\begin{array}{l}\text { Taha tinana } \\
\text { (relating to } \\
\text { physical health } \\
\text { and well- } \\
\text { being) } \\
\text { (Section } 5.1 .4 \text { ) }\end{array}$} & $\begin{array}{l}\text { Physical expression: } \\
\text { - teachers incorporate } \\
\text { movement }\end{array}$ & $\begin{array}{l}\text { Teachers incorporate teacher-initiated and } \\
\text { student-initiated movement. }\end{array}$ \\
\hline & $\begin{array}{l}\text { - teachers show awareness } \\
\text { of physical well-being }\end{array}$ & $\begin{array}{l}\text { Teachers acknowledge and attend to } \\
\text { physical comfort and need. }\end{array}$ \\
\hline & $\begin{array}{l}\text { - teachers focus on } \\
\text { mathematical learning }\end{array}$ & $\begin{array}{l}\text { Teachers incorporate and accommodate } \\
\text { physical movement related to mathematical } \\
\text { endeavour. }\end{array}$ \\
\hline
\end{tabular}


Three sets of characteristics emerged from the observation data within each dimension of the teacher care mathematics whare (Table 5.1). These are illustrated by the three-strand weaving design on the wall panels (Figure 5.1) and are discussed in relation to the relevant dimension. The study findings are discussed in relation to classroom learning in general and with a focus on mathematics learning in particular. Teacher behaviours consistent with more than one dimension are discussed within the most relevant category. A few behaviours particularly pertinent to several sections are discussed in relation to more than one dimension. Finally, the overall effect of behaviours relating to the four dimensions is discussed (Section 5.1.5).

The classroom observation schedule (Appendix 3) was derived from literature relating to teacher care (Chapter Two), consultation, and trials (Chapter Three). The analysis and discussion of Sections 5.1 and 5.1.1-5.1.5 are drawn in the main from the observation data gathered from the lessons of four of the study teachers: the two whose practice most consistently displayed caring teacher acts and the two who least frequently demonstrated caring teacher practice, as indicated by the observation and student data (Section 4.5). Although all teachers showed teacher care, for simplicity in this section, the former are referred to as the 'caring teachers' and the latter as 'less caring'.

\subsubsection{Taha Hinengaro}

Practices that assisted in building safe, purposeful, fun, and engaging learning environments are discussed in this section. Those that showed specific examples of teacher care for mathematics learning are particularly pertinent to this dimension of the model. Due to the fundamental importance of teacher care for mathematics learning to this study, this aspect is discussed in greater detail in Section 5.2.

The caring teachers used a range of strategies to establish and maintain safe and purposeful learning environments. They consistently reinforced firm boundaries and ensured students were aware of their expectations. They created warm, caring atmospheres with a clear focus on mathematics learning. The caring teachers:

- used consistent practices, including clear signals (often using the same wording each time the instruction was given);

I'll know you are ready when your arms are folded and your mouths are shut. (SS)

- were explicit about their practice;

I'm coming around to see your progress. You've got five minutes and then we'll see what we think about one another's ideas. (topic summary task) (SS) 
- allowed for and utilised students' reactions and responses, for example by allowing students to discuss test results to progress their understandings of assessed work immediately after the tests are given back; and most crucially they

- liked and showed respect for their students, for example, by using and accepting gentle teasing (WK).

The giving and receiving of respect as a crucial component of teacher care is an important finding from this study that will be discussed in further detail in Chapters Seven (Section 7.1.2) and Nine (Section 9.1.3).

The behaviours listed above were found to contribute to lessons being calm and organised and students arriving at class expecting and ready to work. The caring teachers generated a sense of urgency for engaging with and completing tasks. These teachers maintained students' engagement by constantly challenging students' thinking (e.g., by adding new rules to a game to change its complexity, adding new ideas about the topic, and using a range of question types). They incorporated a variety of activities into each lesson. The caring teachers:

- involved students in decision making (e.g., in choosing the difficulty of the activities they would attempt (SS) and choosing lesson activities (WK));

- varied lesson activities (e.g., using teacher instruction, brainstorm, a pairs activity, a game, practice examples, one-to-one interactions, textbook work, and homework check, all within the same lesson, (WK));

- incorporated mathematical games and fun lesson starter activities (SS); and

- set consistently high (yet attainable) expectations of work output and success (SS, WK).

The caring teachers had classrooms where student and teacher-initiated humour and laughter were accepted, enjoyed, and managed (also see Section 9.1.4):

I forgot to tell you, whoever wins this won't get anything to eat! (WK)

Less time at the gym sir! (one of WW's students as the door handle fell off)

There's a bit too much frivolity in here. A bit of frivolity is good but there's a bit too much now, you can have some more in a minute. (WK)

Observations indicated that it was not essential for positive effects on the classroom atmosphere that students understood the teacher's humour. It appears that teacher humour indicates to students that the teacher is relaxed and comfortable which seems sufficient in itself to lighten the atmosphere: 
In reaction to a student cough: David, do you know the first sign of bubonic plague? What's bubonic plague sir? (WK and one of WK's students)

Less caring practices relating to taha hinengaro include low expectations of student capability or work output, lack of provision of engaging and/or varied tasks, missed opportunities to acknowledge student humour, acceptance of off-task behaviour, and teacher-directed rather than student-centred practices. Teacher-directed practices are less compatible with the reciprocal links between teaching and learning expressed within the Māori concept of ako (Section 1.1) than are studentcentred practices. It may be that shared responsibility for and participation (of teacher and students) in learning are particularly important in classrooms with comparatively high proportions of Māori and Pasifika students.

\subsubsection{Taha Whānau}

Practices that appeared to help develop a deep sense of community and social responsibility within the mathematics classroom are discussed in this section. They are presented in four areas: nurturing a sense of class community; nurturing students' sense of personal responsibility; teacher care for personal learning needs (emotional, physical, and psychological); and teacher care for students as individuals within their wider family and community contexts.

The caring teachers nurtured students' sense of classroom community by deftly incorporating aspects of their personal identity into their professional identity. They allowed students to know elements of themself as a person by sharing selected personal information in ways designed to enrich learning. Examples included:

- when WK wanted to emphasise the importance of doing homework he explained how he used to do homework when he was their age and why he did it that way;

- in the first lesson of the year, SS told students aspects about herself and her family before asking students to write letters about themselves for her; and

- as an introduction to the probability simulation task into card collecting, SS told students a story about her own child's collection of cereal cards.

The caring teachers showed interest in their students in and outside of their mathematics learning. They:

- capitalised on opportunities to acknowledge shared endeavours in and outside the classroom (e.g., school events); 
lesson before school house sports, WK: Remember there is only one whānau (house group) that wins and that's Kowhai (Yellow)!

lesson after school house sports, a student asks: Mr, did you say Kowhai was going to win?

WK then discussed aspects of the day that involved specific students from the class.

- used inclusive language (e.g., let's see what happens when..., we'll look at that one next..., how many decimal places do we move...);

- showed they prioritise the students and their students' learning (e.g., by explaining to students why they wanted them to work well, following up, and persevering, WK);

- consistently acknowledged students as people (e.g., in greetings, farewells, and teacher and student-initiated discussions);

- used practices that encourage students to share aspects of their own knowledge and personalities (e.g., students helping their teacher with spelling of Māori and Pasifika terms such as 'whānau', having students write and share stories about their mathematics learning);

- participated in learning tasks with their students (e.g., games, SS, outdoor measurement task, WK);

- incorporated activities that encourage a sense of whole class community (e.g., whole class games, whole class discussions); and

- allowed opportunities for students to work together (e.g., in pairs, groups, or informally talking quietly about their work).

The most caring teachers nurtured students' sense of personal responsibility by extending their interest in, and concern for, students outside of the realm of mathematics learning and the lessons they shared. The caring teachers encouraged students' sense of responsibility for themselves and others through:

- discussing life, learning, and behavioural choices within and outside school and possible consequences of these (e.g., behavioural expectations in class, assembly, and other subjects);

You know I don't like staying after school. I know you don't like staying after school. (WK)

- $\quad$ setting clear expectations;

I expect you to do extremely well. (WK)

If it is not neat enough you will do it all again. (WK)

I'm worried about the rude people. (WK); 
- having students swap work for marking (SS); and

- encouraging students' respect for one another and care for the environment (e.g., expecting them to pick up their own classroom rubbish).

As for many teaching strategies, a safe environment and positive teacher-student relationships are necessary conditions for many of these strategies (e.g., students' swapping work for marking) to have maximum effect on students' learning and on their social and emotional development (Darling-Hammond \& Bransford, 2005).

The caring teachers attended to students' emotional, physical, and psychological learning needs in order to maximise learning opportunities. They did this by attending to students' concerns, having spare calculators, equipment, and homework books available for those who could not afford them or had not brought them, and by attending to issues of whakaiti (humility) and whakamā (shyness, embarrassment) (e.g., by praising and reprimanding in safe ways, such as through a quiet one-to-one discussion).

Although three of the study teachers (SS, WW, WK) knew a little about many of the families of their students, very few instances of teachers incorporating this knowledge into lesson times were apparent. All three held roles of pastoral care for their classes, two as deans and one as form teacher, and SS considered aspects of her deaning role to be in conflict with the nurturing role needed to help students learn mathematics. Data related to teachers' acknowledgement of students' families that occurred in quiet one-to-one interactions outside the range of the audiorecorder and earshot of the researcher may have been missed. However, it is possible that the teachers deliberately or subconsciously kept knowledge of students' families separate from the learning context.

Less caring practices relating to taha whānau include those that undermine students' senses of community and community purpose, for example, public admonishment, lengthy times spent in lining students up outside the classroom before the lesson, making mistakes with students' names, and disregarding students' concerns.

\subsubsection{Taha Wairua}

The caring teachers attended to taha wairua in the respect they showed for their students, by using praise, providing timely assistance with and feedback about learning and other matters, and by making multiple opportunities for one-to-one interactions with every student every lesson. The caring teachers: 
- acknowledged students as individuals, e.g., by reflecting their knowledge of the students within and outside the classroom, and thanking them;

Thank you for being so courteous. (WK)

Selina, it is unusual for you to be late. (SS)

Can anyone beat Pepe today? (SS in starter game)

- told students that they respect them and exhibited behaviour consistent with this (e.g., apologising when appropriate, WK);

- encouraged and respected students' self-assessment of their progress and work level;

I think you need to move away from Steven. Decide please and if there is any more talking we'll find something interesting to do at lunchtime. (WK)

- explained their practice (e.g., by signalling what to expect during the lesson or in the next few lessons and through explaining their rationale for teaching decisions) thereby acknowledging and encouraging student ownership of their learning: and

I want to see your working out as well as your answers because that shows your thinking. (WW)

- used praise for individuals and the whole class, including using opportunities to praise and encourage alienated students:

Herewini is my star today! (SS)

I went around the class and asked people what they had learnt today and they all said something, so that's good! (SS)

I know you guys are great with homework. I have it all recorded from last year. (WK)

The caring teachers employed multiple methods of one-to-one interaction with all students every lesson (also see Section 9.1.1). Typical strategies included using students' names, making multiple opportunities for assistance and checking on individuals' progress (e.g., book work, homework, progress with learning tasks, review questions), attending to detail (e.g., noting who has done the homework, who has work completed, and who is making particularly strong or weak progress).

The caring teachers facilitated and encouraged students' sense of personal mathematical identity by acknowledging learning progress, setting appropriate work, relating learning to students' lives, and being encouraging regarding students' efforts. They provided multiple opportunities for students to feel mathematical 
success and satisfaction and access assistance with their work. They publicly and privately acknowledged students' mathematical efforts and successes and provided timely and specific feedback (e.g., immediate feedback of test results (SS), and acknowledging those wanting help when they could not assist immediately):

Derek, how are you going? l'll be down there in a minute. I'm not neglecting you. (WK)

How many got this line right? this line? this line? (SS)

The less caring teachers provided fewer opportunities for students to share responsibility for their learning and enter into classroom decision-making. They made less provision for student enjoyment of their learning, mathematical success, and student interest in the learning tasks. They devoted less lesson time to one-to-one interactions and did not interact individually with particular students over many lessons. One-to-one interactions tended to be less positive than those of the caring teachers, for example these teachers were more often focused on discipline issues. At times, the praise given for students' work level or quality was invalid. Although well intentioned, invalid teacher praise (e.g., praising students for on-task behaviour when they have been largely off-task or praising progress where there is very little progress) appeared to indicate to students that not doing their best was acceptable.

\subsubsection{Taha Tinana}

The tinana side of the tapa wha was interpreted as including all behaviours pertaining to students' movement and physical well-being. A traditional secondary school mathematics lesson holds few opportunities for students to move around the room. Very few observed lessons over the entire study involved much student movement from their desks. Examples of opportunities for student movement from lessons with the most caring teachers included students writing on the board, standing up as part of a game or to indicate their learning or effort (e.g., success in homework or confidence with integer work), playing games which involved movement (e.g., paper, scissors, rock game for probability), learning tasks carried out outside the classroom (e.g., Section 5.3), and being able to move around the room to obtain assistance from peers.

Care for students' physical well-being was shown by the most caring teachers through:

- attending to the classroom environment, e.g., through ensuring sufficient light, fresh air, and heat; 
- discussing different physical needs in relation to the classroom furniture, e.g., pointing out that the chairs were of different heights and therefore would suit different students (SS);

- allowing students to go to the toilet;

- $\quad$ sending for water when a student had the hiccups (SS);

- acknowledging the effects of the environment on students' learning;

I know it is hot in here and I know it is last period and you guys are doing really well. (WK)

- $\quad$ showing concern for students physical well-being and injuries; and

If you want to be an athlete you know what you need to do, don't you? (WK to student who is a keen athlete but an occasional smoker)

- encouraging students taking initiative in relation to physical well-being (e.g., by allowing students to open windows and drink water in class).

Common teacher behaviour in relation to students' physical space was that of enhancing teacher-student proximity when teachers were assisting students one-toone or one-to-group. Many teachers leant towards the students when helping them (SS, UU, UM) and two teachers (WW and WK) came even closer to their students by kneeling close to the students' desks. That students were comfortable with this close teacher proximity is an indication of the strength of the teacher-student relationships and the levels of trust between the students and their teachers. The closest teacher proximity in one-to-one and one-to-group assistance resulted in teachers and students having their heads and eyes at the same physical level or with the teachers' heads slightly below their students' heads. This affirming teacher behaviour appeared to assist direct teacher-student interaction by:

- psychologically placing the student as a significant participant;

- showing the student that the teacher was there willing to assist; and

- enabling greater privacy in these interactions than would have been possible with greater distance between teacher and student.

Less caring teacher practices relating to students' physical well-being often resulted in some type of student discomfort. They included teachers insisting on school uniform regulations despite negative effects on student well-being (e.g., a student becoming cold as a result of the removal of a non-regulation jersey), making students wait to go to the toilet, and lack of attention to the comfort of the classroom 
environment (e.g., excessive heat or glare). On the whole these teachers less frequently included learning activities involving student movement.

\subsubsection{A holistic view of the tapa wha model of teacher care}

The two teachers who emerged as consistently showing care for their students' learning did so through their words, actions, body language, and their preparation for teaching. Within the context of mathematics learning, these teachers most consistently attended to ways of creating positive learning environments, developing strong learning communities, making opportunities for students to express their personal identity, and allowing for students' physical expression.

The observations indicated that students are generally tolerant of minor gaps and lapses in caring teacher behaviours. By Year 10 all students have experienced many different teaching styles and many different learning environments. This is a likely contributor to their tolerance of gaps in teacher care across the sides of the tapa wha. However, teacher care for mathematics learning, particularly when it is effectively translated into student confidence in their mathematical progress appears essential for strongly felt student perceptions of teacher care. Strongly caring teacher behaviours for the other three sides of the tapa wha without effective caring behaviours for mathematical learning seemingly, for most students, are insufficient to ensure an optimal disposition for learning because they are constantly undermined by students' frustration at their lack of mathematical progress.

Another consideration of students having been 'enculturated' into the schooling system is the seeming acceptance of a psychological distance experienced in classrooms between students' homes and families and school. How teachers can better address this area of the taha whānau in their mathematics teaching is an important area for further study.

Consistency and reliability seem important factors in shaping students' perceptions of teacher care. All of the teachers voiced in their interviews that they cared for their students' learning and cared for their students as people (Chapter Six). However, some teachers were more able than others to demonstrate this convincingly to their students. Many caring practices were used by all teachers (e.g., safe questioning practices, praise) but these were less effective or completely undermined when teachers were less persistent with, or had major gaps and lapses in, caring behaviours. Teacher practices that appeared to particularly undermine students' perceptions of teacher care included: 
- consistently underestimating students' capabilities in terms of the quantity, quality, and/or the level of difficulty of the work;

- $\quad$ not keeping the students on task (leading to students being bored, knowing they were not learning, and feeling their teacher did not care whether or not they were learning);

- giving conflicting messages (e.g., spending time reviewing school rules at the start of the lesson but having limited success in insisting these were followed in the lesson);

- not acknowledging student effort;

- inappropriately praising substandard effort;

- relying on punitive discipline and using practices seen as unfair (e.g., sending a student out of the classroom);

- not explaining their actions and teaching decisions;

- $\quad$ not providing assistance when needed; and

- not giving individuals specific feedback on their progress.

\subsection{Care for mathematics learning}

This section focuses on mathematics teaching and learning. The discussion in this section is derived from analysis of the lesson observations and the lesson audiorecordings. The analysis methods are described and four focus areas are presented: supporting new mathematical learning (Section 5.2.1); reinforcing mathematical learning (Section 5.2.2); using contexts relevant to students (Section 5.2.3); and giving encouragement, assistance, and feedback (Section 5.2.4). Transcripts of short lesson segments and other examples of observed teacher practices are included to exemplify the discussion. All names within the transcripts are pseudonyms. Key findings from this section regarding teacher care for mathematics learning are summarised in Section 5.2.5.

Mathematics teaching and learning is a key component of the taha hinengaro of the teacher care mathematics whare (Table 5.1) and analysis of the data is presented using a new framework that incorporates elements identified by Bishop et al. (2003). Begun in 2000, the Ministry of Education funded research and professional development programme, Te Kotahitanga (Unity), is aimed at improving the engagement and achievement of Māori students in mainstream secondary schools. An "effective teaching profile" (Bishop et al., 2003, pp. 97-118) was one outcome of the initial research project. Professional development using the profile was carried 
out with four schools from 2001, eight more schools from 2002, and a further twentyone in 2006, bringing the total number of Te Kotahitanga schools to thirty-three. Research indicates the professional development has impacted positively on student achievement. ${ }^{25}$

Developed from data collected from interviews with students, parents, teachers, and principals, the 'effective teaching profile' describes the characteristics, dispositions, and behaviours of effective teachers of Māori students. These teachers strongly reject deficit theorising, are committed to enhancing Māori students' achievement, and know how to accomplish this. They attend to:

1) Manaakitanga: They care for the students as culturally-located human beings above all else.

2) Mana motuhake: They care for the performance of their students.

3) Ngā tūranga takitahi me ngā mana whakahaere..$^{26}$ They are able to create a secure, well-managed learning environment.

4) Wānanga: They are able to engage in effective teaching interactions with Māori students as Māori.

5) Ako: They can use strategies that promote effective teaching interactions and relationships with their learners.

6) Kōtahitanga: They promote, monitor and reflect on outcomes that in turn lead to improvements in educational achievement for Māori students. (Bishop et al., 2003, p. 98)

Bishop et al.'s (2003) characteristics 1) - 3), 5) and 6) link well with the analysis discussed using Durie's whare tapa wha model (Section 5.1). The remaining section of 'the effective teaching profile' (i.e., Wānanga), further detail within Ako, and four further salient lesson elements that emerged from the study data (humour, reflecting personal identities, praise, and teacher questioning) were used to create a framework for analysing the audio recordings of selected lessons (Table 5.2). The framework includes four groups of caring teacher practices: supporting new mathematical learning; reinforcing mathematical learning; ensuring the relevance of mathematical learning; and giving encouragement, assistance, and feedback. A template incorporating all sections

25 In level 1 NCEA from 2005 to 2006 an increase of 16.4 percentage points was gained by students in the first 12 Te Kotahitanga schools, and 11.2 percentage points for level 1 students across all Te Kotahitanga schools (compared to 8.9 percentage points nationally) (Ministry of Education, n.d.).

26 A subsequent version of the 'effective teaching profile' (Bishop, Berryman, Cavanagh \& Teddy, 2007) has replaced this descriptor with 'whakapiringatanga' described as "a process wherein specific individual roles and responsbilities are required to achieve individual and group outcomes" (p. 27). 
of the framework was used to record (in two and a half minute time intervals) the lesson elements present during the lesson (e.g., Appendices 14 and 15) ${ }^{27}$.

\section{Table 5.2: Framework used to analyse lesson audio recordings}

Note:

* indicates elements drawn from 'Wānanga' (Bishop et al., 2003),

** indicates elements drawn from 'Ako' (Bishop et al., 2003)

\begin{tabular}{|c|c|}
\hline Caring Practices & Lesson Elements \\
\hline \multirow{4}{*}{$\begin{array}{l}\text { Supporting new } \\
\text { mathematical learning } \\
\text { (Section 5.2.1) }\end{array}$} & Co-construction, ${ }^{*}$ negotiation of learning \\
\hline & Prior knowledge $\mathrm{e}^{*}$ \\
\hline & Instruction, ${ }^{*}$ teaching rather than telling \\
\hline & $\begin{array}{l}\text { Teacher questioning, questions from teacher to whole class or } \\
\text { to individuals }\end{array}$ \\
\hline \multirow{3}{*}{$\begin{array}{l}\text { Reinforcing mathematical } \\
\text { learning } \\
\text { (Section 5.2.2) }\end{array}$} & Narrative ${ }^{* *}$ committed, connected, participatory teaching \\
\hline & Co-operative learning ${ }^{\star \star}$ \\
\hline & Oral language/literacy, ${ }^{* *}$ orally rich atmosphere \\
\hline \multirow{5}{*}{$\begin{array}{l}\text { Ensuring relevance of } \\
\text { mathematical learning } \\
\text { (Section 5.2.3) }\end{array}$} & Integrated curricula, ${ }^{* *}$ context matching students' interests \\
\hline & Student-generated questioning ${ }^{\star *}$ \\
\hline & Differentiated learning ${ }^{\star *}$ \\
\hline & Self, involving personal identities \\
\hline & Humour \\
\hline \multirow{4}{*}{$\begin{array}{l}\text { Giving encouragement, } \\
\text { assistance, and feedback } \\
\text { (Section 5.2.4) }\end{array}$} & Feed-forward academic ${ }^{*}$ \\
\hline & Feed-back academic ${ }^{*}$ \\
\hline & $\begin{array}{l}\text { Ako,** reciprocity, teacher as partner in conversation of } \\
\text { learning }\end{array}$ \\
\hline & Praise \\
\hline
\end{tabular}

In some respects analysis of the data from the audio-recordings was less subjective than that of the observational data (e.g., verbatim discussions could be analysed) and in other respects more subjective (e.g., the body language which accompanied the discussions could not be taken into account). Other limitations to this data analysis method include that:

- the analysis was totally dependent on the data that could be recorded (a subset of the classroom conversations, instructions, explanations, and questions);

- the recording equipment was able to capture all interactions in whole class teaching and teacher-student interactions closest to the microphone but not those further away;

- the contexts of mathematics problems that were discussed can be ascertained but other questions on the whiteboard, worksheets, and textbooks cannot; and

\footnotetext{
27 The analysis method used was adapted from an audiotape analysis process used by
} Thorpe (2007). 
- on a small number of occasions (estimated as fewer than five in total), student behaviour changed from normal practice due to the presence of the microphone.

Much detail can be ascertained from the recordings in spite of these limitations. Examples include the amount of time teachers spend on behaviour management and modes of instruction, the apparent confidence with which students respond to questions, the types of questions asked by students, and teacher persistence. Using the framework allowed comparison of teachers' practices. In order to focus on lessons using the most typical practice, only the data from lessons later in the school year were used. Audio recordings analysed using the framework included a typical lesson for each of the study teachers (e.g., Appendix 14), and examples of an exemplary lesson from each of the two most caring teachers, SS (e.g., Appendix 15) and WK (Figure 5.6). Shaded boxes were used to indicate each lesson segment that included at least one instance of each lesson element. Boxes were not shaded if the element was not implemented at all or, in the researcher's view, was not effectively implemented (e.g., when teacher instruction was taking place but the class was largely off-task).

\subsubsection{Supporting new mathematical learning}

The study teachers supported new mathematical learning using a combination of coconstruction of new learning, making use of students' prior knowledge related to the new learning, instruction, and questioning. This section will discuss each of these in turn. Excellent examples of the use of co-construction, instruction, and teacher questioning are found within the transcript of nine and a half minutes of one of UM's lessons (Figure 5.2). The excerpt is taken from a lesson on graphing linear equations of the form $y=m x+c$. In the transcript UM is reinforcing the new concepts he has introduced earlier in the lesson with problems related to real life that require finding the equation of a given line and graphing a line from a given equation.

\footnotetext{
UM: OK, now for this one you have to write the equation. Now you have one minute everybody, no hands up please, to work out the equation for the 45-seat bus. You look for the y-intercept, you look for the gradient, and then you write the equation. Try your best. Just wait, I'm not taking answers yet.

Tama: But this is easy, Mr.

UM: Yes, it is easy I know, but it might be even harder if we don't wait.

(students are discussing the question in pairs)

Steph: $\mathrm{Mr}$, is it $\mathrm{c}$ and $\mathrm{t}$ or $\mathrm{x}$ and $\mathrm{y}$ ?

UM: $\quad c$ and t please.

One student to another: You go up 200 and across 50.

UM: Ready? OK, time's up. Did someone do it? I know Tama did. He's so fast, he's trying to prove he is the fastest one. And it worked this time. Thank you! Oh, that's good.
} 
Regan: Is it $\mathrm{c}=(200$ over 100$) \mathrm{d}+100 ?$

UM: Excellent, that's the first one. Did anyone get a different one?

Mere: I got $\mathrm{C}=2$ over 1 times d plus 100 .

John: It's the same! (students discuss whether or not it is the same)

UM: Ok, another one?

John: It is the same! (students keep discussing whether or not it is the same)

UM: Why is it the same? Or why is it not the same?

(class discussing with each other and agreeing the equations are the same)

UM: OK, one second, I can't hear what somebody's saying.

Mere: Can we use $x$ and $y$ ?

UM: I'm not sure, I don't think it is something that is perfectly wrong to use $x$ and $y$ but let's look at the question. They use $c$ and $t$ so let's use $c$ as distance and t as time for this one. That's good, that's a very good start for today, now we move on and let's look at question number 9 . It is a long one, an NCEA one, so try your best.

(quiet while students try the question)

UM l'd like to see more students involved. I have seen some are always giving answers and not the others.

(quiet while students continue with the question)

UM: Twenty seconds left.

(quiet)

UM: OK, time's up. Now, you can see it is about calling overseas and we have three different types of calling cards. We have three different options, one called 'Anytime', and the other called 'Keep in Touch'. Now part a), you have time to read a), for the 'Anytime' card we can see the cost per call is described by the equation $c=5 t+20$. Now let's start with Liam, what is the first thing we do when we start with an equation like $\mathrm{c}=5 \mathrm{t}+20$ ?

Liam: We start on 20?

UM: Yes, excellent, we start on 20, thank you very much. This is the y-intercept. OK, that's because even before we start our phone conversation with somebody we start with a charge of 20 cents. After that we are charged by the minute. OK, what do we do next please Stuart?

Stuart: We start on 20. You go up 5 for every one minute across.

UM: That gets you to 25 .

Nathan: I didn't do that. I changed the equation to 20 over 5 t.

UM: I'm not sure it is easier. I didn't think about it, but it seems complicated. It will be something easier than that. We don't have to complicate maths. Susan, your turn.

Susan: I replaced t with 30 and got $30 \times 5$ and then worked out another point on the graph with $\mathrm{t}=30$ and $\mathrm{c}$ as 170 .

Figure 5.2: Transcript from an excerpt of a lesson given by UM

Co-construction is evident in the way UM and the class interact about the learning, with the excerpt reading much like a conversation. The students spontaneously ask for clarification of aspects of the problem and he replies, at times turning their questions to him into questions for the whole class. An example of this is evident when students are puzzling over whether or not the two different equations are two ways of writing the same equation. UM allows time for the students to puzzle it out 
together, not moving on to the next question until there is general agreement and understanding. Similarly, in his teaching WK also involved students in mathematical thinking and argumentation centred on one another's ideas:

I'll let you two argue it out, one of you is correct and the other one isn't. (WK)

How many people concur with this working? (WK)

In a subject where students can quickly lose confidence, it is important that obstacles to students' progress are minimised. One way to minimise these obstacles is to ensure that the prior learning required for understanding ideas or solving problems is in place before students meet a new mathematical idea or attempt a problem using the new idea. Many examples of teachers both strategically and spontaneously trying to reactivate the prior knowledge required for students' new learning were observed, most often at the beginning of lessons. Examples of strategies used to reactivate prior knowledge at the beginning of lessons included:

- using targeted review questions, (e.g., questions involving substituting values into formulae before later measurement work requiring formulae use (WK));

- reminding students of how to use the calculator functions they would need for the day's problems (UU); and

- brainstorming all that students could remember about an idea at the start of the topic (e.g., using fractions, UM).

Teachers were also observed reminding students of useful prior knowledge during the lesson as needed.

UM's extensive use of questioning (e.g., Figure 5.2) is an example of teacher 'instruction' (teaching rather than telling). He provides pace and structure to the instruction session by keeping the class working together through each section of the problem, setting time limits, reviewing, discussing, and giving feedback on each section before introducing the next. This step-by-step process enables UM to move around the classroom to check students' books for progress and understanding, and answer students' queries.

UM reinforces new learning by giving hints of what needs to be done (e.g., "You look for the y-intercept, you look for the gradient, and then you write the equation."). These pointers serve as reminders of the newly learnt ideas and show how UM is encouraging students to think for themselves how they should do this rather than telling them exactly what to do (i.e., find where the line cuts the y-axis, calculate the gradient using the rise over the run, put the numbers in place of the ' $m$ ' and the ' $c$ ' in 
the general equation $y=m x+c)$. Lesson observation data indicated that the provision of a variety of suitable mathematics problems was essential for keeping students thinking and working mathematically and therefore for supporting new learning.

UM used both written and oral questions (e.g., Figure 5.2) directed at the whole class and oral questions directed at individuals. He draws all answers to the problem from the students and deflects attention away from erroneous methods that may confuse others, such as when Nathan was trying to explain a dubious method for graphing the equation. Students readily replied to UM's questions, an indication that they felt comfortable and safe to answer. Other safe questioning practices observed across study teachers' lessons included:

- asking questions to the whole class rather than 'cold-calling' (Chapter Two);

- each student being able to anticipate which questions they would be called on to answer (e.g., by asking students around the classroom in a predictable way);

- allowing students to confer with others before replying in front of the whole class; and

- the teacher having students chant answers together (e.g., to finish his unfinished sentences, WK).

\subsubsection{Reinforcing mathematical learning}

The observed lessons provided many examples illustrating that learning experiences that involved teacher and student participation, interaction between students (e.g., cooperative learning, conferring between students, mathematical games), and the use of written and oral language for embedding new learning generated higher proportions of on-task behaviour and more student enthusiasm. The lessons using such learning experiences were closer to achieving a sense of 'mālie' (Manu'atu, 2000, Section 1.2) than the more traditional lesson style characterised by teacher exposition followed by practice problems from a worksheet or textbook.

The typical format of SS's lessons included diverse strategies for reinforcing mathematical learning, such as:

- a game (e.g., 'buzz', a whole class game involving the students and teacher standing in a circle and counting around the circle from one, replacing any 
multiple of a given number (e.g., 7) with the word 'buzz'. Students who made a mistake sat out until the next round of the game);

- review questions based on recent work;

- whiteboard problems for students to work on with a peer (e.g., equations of straight line graphs, gradients, $y$-intercepts);

- linking the new content to a context in students' experience (e.g., discussing gradient in terms of the steepness of children's playground slides);

- revisiting new learning in different ways during the lesson (e.g., teacher exposition and questioning, students working on their own from textbook questions);

- initiating and discussing student-generated rules (e.g., for finding the gradient and $y$-intercept of a line from an equation); and

- reviewing lesson content at the conclusion of the lesson.

Having already worked with others on similar problems, students readily consulted with their peers when they required assistance with the textbook questions.

The formal use of cooperative learning experiences (in contrast to individual tasks and tasks students worked on with others) was seldom sighted during the lesson observations. Roughly $5 \%$ of lessons included these activities and only three study teachers (SS, WW, and WK) were observed using cooperative learning experiences. However, a lesson focused on reinforcing probability concepts provides a good example of participatory teaching using cooperative learning and requiring use of oral mathematical language. Many in the class already knew the game 'rock, scissors, paper' in which players simultaneously make a rock, scissors, or paper with their hands. SS asked the class to get into groups of three and decide on who would be Players $A, B$, and $C$ to play a modified version of the game. The rules of the modified game used were that:

- A would win if all three players made different objects (one rock, one scissors, one paper);

- B would win if two of the group had the same object; and

- C would win if all three made the same object (all rocks, for example).

Students were asked to play together and to investigate as a group whether or not the new game was fair. Later, having decided the game was not fair; groups were 
challenged to modify the game to ensure that it was fair. SS joined a group who needed a third player (thus enhancing a sense of community, Section 5.1.2). The context and activity motivated the students. It was sufficiently challenging and open that students wanted to stay in their groups to work out ways to modify the game with others rather than work on their own. Solving the problem required brainstorming of ideas, negotiation, and trial and error. Some groups used theoretical probability tools (e.g., tree diagrams) to explore the problem. Once all groups had made progress with creating a more fair game, each group shared their strategies with the whole class.

In another lesson SS used story writing as a means of reinforcing new learning. Students were to write a story of between fifty and one hundred words that involved measurement conversions, then share their stories with the students sitting in their group. Each group was asked to choose one of their stories to be read to the whole class. The stories (e.g., Figure 5.3) varied greatly. Sharing of the stories generated much mathematical thinking, laughter, and enjoyment. The story-writing task enabled students to embed their learning about measurement conversions whilst providing them with the opportunity to be creative, use humour, and reflect themselves in their work. Sharing the stories helped students acknowledge their peers' work and individuality, and enhanced their sense of classroom community.

SS: $\quad$ Well done everyone. It is very good to be able to write maths in a story and show we use it every day. I love doing these things myself. I love rhymes. I love writing. I think Samuel's one is really quite clever. I want you to tell me at the end what is clever about his story. I want you to listen quietly.

Long, long ago there lived the tallest line called one kilometre. One kilometre had a son called one thousand metres. One thousand metres lived many measurements away and had a daughter called one hundred thousand centimetres who bore a girl called one million millimetres.

What's Samuel done that is quite clever?

Aroha: All the names are measurements.

SS: Yes, but what is it about the names that is so clever?

Steven: All the measurements equal the first one.

SS: $\quad$ Yes, that's right, well done Samuel, a very good story. He's made a whole family using the same measurement; one kilometre. Well done Samuel!

Figure 5.3: Transcript from an excerpt of a lesson given by SS

Teachers used a range of ways to emphasise the importance of homework in reinforcing mathematical learning; daily checking that homework had been completed (SS, WK), acknowledging students who had completed their homework 
by having them stand up, by stamping their books (SS), by writing letters to the students' parents acknowledging excellent work (UU), reviewing answers (SS, WW, WK, UM), and expecting students to return at morning tea or lunchtime to complete unfinished homework (SS). On one occasion WK was particularly concerned over the number of students who had not completed their homework. He addressed the issues with the class. Firstly he signalled they would have a class discussion about homework: "After marking the review questions I am going to discuss homework." After the review questions WK asked the students to calculate how many hours there are between 3.15pm Friday and 11am Monday, beginning the discussion about homework by changing the context into a calculation problem. Students slowly worked out for themselves why he had asked them to carry out the calculation. He acknowledged those who had done their homework and pointed out that those who had not done it had not used any of their calculated $913 / 4$ hours to get it done. He continued by expressing his expectations and his concerns for the learning of those who regularly did not complete homework tasks. This creative approach to discussing homework expectations encouraged students to consider for themselves their decisions about completing homework tasks and make decisions about homework completion for themselves in a non-threatening and informed way.

\subsubsection{Ensuring relevance of mathematical learning}

It is widely accepted that students are more motivated to learn when they feel the content is personally relevant. This can be achieved when students see the usefulness of the learning for their everyday lives, when the content or context matches their interests, or they can see themselves in their learning in some way. An overall impression from the lesson observations was that lessons in which students perceived the learning to be personally relevant included more studentgenerated questioning, more opportunities for students and the teacher to reflect their personal identities, and more teacher and student-generated humour.

One series of lessons in particular exemplified many ways of enhancing the relevance of mathematical learning for students. WW gave his class an investigation into surface area and volume (Figure 5.4) that required them to work together to approximate the surface area and volume of their own bodies by selecting and justifying models for different body parts, taking measurements, selecting and using formulae, carrying out calculations, determining the accuracy of their approximation, and presenting their results as a poster. 


\section{Finding approximate values for the surface area and volume of your body}

Your task is to find approximate values for the surface area and volume of your body. These two measurements have importance in the biology of the human body. Use the table to record your measurements.

You will need to use common objects as models for the different parts of the body, for example your neck could be represented by a cylinder.

You need to justify your choice of each model you use and for each, estimate the accuracy of the calculated measurements. Include your reasoning.

The work is to be presented in a poster (so don't be too personal with your measurements!)

All measurements are to be in $\mathrm{cm}$ and recorded to the nearest $\mathrm{mm}$ (i.e., to $1 \mathrm{dp}$ ).

\begin{tabular}{|l|l|l|l|l|l|l|l|}
\hline $\begin{array}{l}\text { Body } \\
\text { Part }\end{array}$ & Model & Radius & Height & Width & Length & $\begin{array}{l}\text { Surface } \\
\text { Area }\end{array}$ & Volume \\
\hline head & & & & & & & \\
\hline neck & & & & & & & \\
\hline torso & & & & & & & \\
\hline leg & & & & & & & \\
\hline etc. & & & & & & & \\
\hline
\end{tabular}

Figure 5.4: Measurement activity

The students were immediately engaged by the context because the activity focused on finding out something about them. Students needed to work together to carry out the measurements to begin the activity. Having begun together they tended to continue with the rest of the activity together, discussing and negotiating the best models to use, their rationales for the choices, and the likely accuracy afforded by the models. Students were able and encouraged to make many decisions about their learning in this activity, for example mathematical decisions such as which models to use, decisions about who to work with, the equipment they would use, how to approach the task, and how to design their poster. For example, when asked for a quick way to find the total surface area, WW replied:

You have to decide. These are the decisions you have to make.

The task was consistent with the characteristics of 'rich mathematical tasks' (Ahmed, 1987 ) in that it was accessible to all and able to be approached with different levels of mathematical sophistication, inherently allowing for differentiated learning. The activity style enabled the teacher to move around the pairs and groups of students providing targeted guidance and assistance and more sustained personal contact (one-to-one interactions) than could be achieved in a lesson using predominantly teacher exposition and whole class practice activities. Furthermore, the activity's context and teacherstudent proximity facilitated student-generated humour: 
Apete, please stop eating. Wait until break for that please. (Teacher)

But I'm trying to add to my volume Mr! (Adam)

Yes Apete, I can see you are! (Teacher) (Teacher and students laugh together.)

One lesson observation in particular highlighted that a good learning experience is in itself insufficient to ensure students' interest or engagement. In this lesson students were given an investigative task in which they were to take measurements from everyday circular objects to estimate $\pi$. The richness of the task was undermined in part by the teacher being unsuccessful in engaging the students' interest and enthusiasm.

In contrast, the volume and surface area activity (Figure 5.4) was successful due to factors related to the students' everyday mathematics learning with WW and to how the activity was presented. In their mathematics lessons students were accustomed to:

- the frequent use of investigative learning experiences;

- positive teacher-student rapport;

- the emotional and psychological comfort of the class environment; and

- the teacher's expectations of their work.

Student engagement was further enhanced by WW's introduction of the activity. He gave an explanation of the mathematical purpose of the task, how the task related to their previous work and the current unit, and clear expectations of how students would work together and how much work they would complete in the lesson. He used questioning to remind students of desired behaviour and was responsive to students' questions and needs (e.g., by providing equipment and poster materials).

The lesson observations provided some excellent examples of student-initiated questioning during teacher exposition, in particular for WW, WK, and UM. At these times students were actively involved in sense-making, the learning created during animated conversations between the students and teacher. The students' questions showed that they were listening, thinking about, and exploring the new concepts and techniques, and that the classroom environment was conducive to students posing questions as soon as they crossed their minds. WW's lesson on solving equations (see excerpt in Figure 5.5) provided an example of how student-generated questions were welcomed and answered. Furthermore, it shows WW spontaneously linking to students' prior learning (i.e., equations with pronumerals on one side of the 
equals sign only) to progress their understanding and encouraging students to take responsibility for their own learning (e.g., by asking them to make their own notes for later study and choose a method for solving the problems).

(The problem being discussed is $6 x+19=41+4 x$ )

WW: What we are going to do first is get rid of the numbers from that side. Mathematically what am I going to do with the 19 to get rid of it from the left-hand side?

Student: Take it away.

WW: Yes, and we must do it to both sides, so what's $41-19$ ?

Student: 22

Student: You write fast, Mr!

WW: $\quad$ OK, so now we have x's on both sides still. All right. Now, so for one part of the problem we've got rid of the number from this side, now we need to get the other number across? What is the opposite of plus $4 x$ ? How can I move the $4 x$ ?

Student: Take away $4 \mathrm{x}$, it gives zero.

WW: I must do it on this side as well. What am I left with?

Student: $2 x$

Student: Wait, $2 x=22$, so what does that mean?

WW: Two times $x$ is twenty-two so how much is $x$ ?

Student: Oh yeah! (the students giggle)

WW: So now we want to write these out without drawing sets of scales all the time.

We want the numbers just on this side so we've got to move them from the other side.

Student: Can you write them like the other one down there? (points to example on the board) Like the 19, take away the 19 ?

WW: OK

Student: If we wrote on a test like this would you understand it?

WW: Yes

Student: OK

Student: Can we use guess and check?

WW: You can use guess and check but it will often take longer. You must keep the equation in balance. Now, all l've done is put some of the key points up here on the board. Now you write down as much as you need in your own notes about how to do these so you have your notes to go back to later to know what to do when you get to these problems. We're going to get the numbers on the right-hand side and the letters on the left.

Student: What's that called Mr?

WW: Solving equations, in other words, finding out what the letter is equal to. It is the same as we've been doing before except now we've got the x's on both sides of the equals sign. You can also try using the scales model in case it helps you to understand it better. (several minutes of quiet while students make their notes and solve the problem.)

If you've finished that, there's another problem on the board. I don't want you using guess and check. I want you using the written form and maybe the scales.

Student: Does it matter if the numbers are on each side? Could you put the $\mathrm{x}$ on the righthand side?

WW: That's fine, but when you write your answer, put it with the $x$ on the left.

Student: Mr, can we do it the other way around with the numbers on that side?

Student: Can you put that as the answer though?

WW: It is still right, but the convention is to put the letter on the left.

Figure 5.5: Excerpt from a lesson on solving equations 
In an attempt to cater for the wide range of ability and prior knowledge in his class, ST consistently seated the most able students of the class together, setting them more challenging work than the rest of the class. Similarly WK often had less challenging work prepared for several students who were working at a lower ability level than the majority of his class. The students given alternative work were consistently on task and discussed their work with each other and with the teacher.

\subsubsection{Giving encouragement, assistance, and feedback}

The lesson observations and recordings provided a range of ways in which teachers show care for their students' mathematics learning through providing encouragement, assistance, and feedback. Four of the study teachers made clear statements of praise for students' mathematical thinking or explanations (SS, WW, WK, UU). Most were clearly available to help students and would persist until students' difficulties were overcome. Teachers SS, WW, WK, and UU discussed the importance for students' futures of their achieving well in mathematics, encouraging them to do their best. Care for their students' mathematics learning was shown by teachers when they:

- encouraged students to work hard, persist through difficulties, and do well;

- acknowledged mathematical effort and success and enabled affirmation of mathematical thinking and achievement by peers;

- sought out those needing assistance and provided timely help; and

- $\quad$ knew and respected how individuals like to receive feedback.

Teachers also showed care for their students' mathematics learning by:

- providing reassurance;

The only way to get into algebra is to do heaps of it and to make it your friend. (WW)

- cajoling;

Pania, you promised me you would work well last week. Oh, there is the misunderstanding! I thought you meant every week! Please promise again! (UM)

- using humour to lift spirits;

Thank you for your help Mr. (Darcy)

Of course, it is free of charge! (UM)

Right, now you are allowed to pay attention and you are allowed to be quiet. (UM) 
- providing specific feedback about learning; and

You will have to work faster than this in the test - in the test you will have to do two whole constructions in one period. (UM)

- providing specific feedback about behaviour;

Steve, there's a certain point when my patience runs out. (WW)

Aspects of teacher encouragement, assistance, and feedback are discussed further in other parts of this chapter (Sections 5.1.3 and 5.3).

\subsubsection{Discussion}

The profiles of the audio recordings of the lessons of the most caring teachers were markedly different to those of teachers with less caring practices, including more lesson elements from the framework (Table 5.2) used more often (e.g., Figure 5.6). Greater frequency of the desired elements appeared to be more easily achieved in lessons centred on mathematically rich tasks (e.g., investigations). Such tasks necessitate or encourage co-construction, use of co-operative work, oral language, differentiated learning and, in some cases, an integrated curricular focus (in line with students' interests and background knowledge).
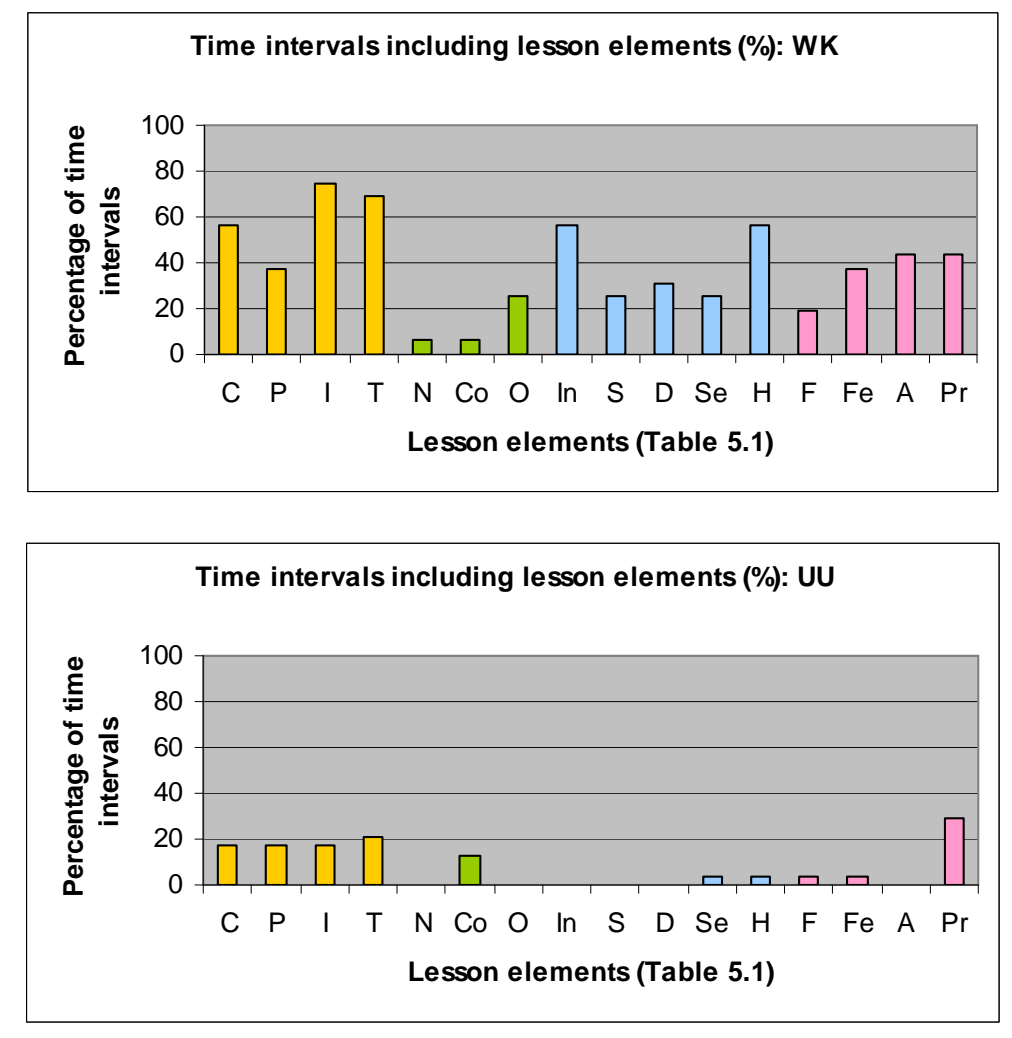

Figure 5.6: Percentages of lesson time intervals incorporating lesson elements for WK (excellent lesson) and UU (typical lesson) 
Key:

Note: Shading has been used to highlight the four types of practice.

\begin{tabular}{|l|c|l|}
\hline Caring Practices & Code & \multicolumn{1}{c|}{ Lesson Elements } \\
\hline \multirow{4}{*}{$\begin{array}{l}\text { Supporting new } \\
\text { mathematical }\end{array}$} & $\mathrm{C}$ & Co-construction, negotiation of learning \\
\cline { 2 - 3 } & $\mathrm{P}$ & Prior knowledge \\
\cline { 2 - 3 } & $\mathrm{I}$ & Instruction, teaching rather than telling \\
\cline { 2 - 3 } & $\mathrm{T}$ & Teacher questioning, questions from teacher to whole class or to individuals \\
\hline $\begin{array}{l}\text { Reinforcing } \\
\text { mathematical } \\
\text { learning }\end{array}$ & $\mathrm{N}$ & Narrative, committed, connected, participatory teaching \\
\cline { 2 - 3 } $\begin{array}{l}\text { Ensuring } \\
\text { relevance of } \\
\text { mathematical }\end{array}$ & $\mathrm{Co}$ & Co-operative learning \\
\cline { 2 - 3 } learning & $\mathrm{O}$ & Oral language/literacy, orally rich atmosphere \\
\cline { 2 - 3 } & $\mathrm{In}$ & Integrated curricula, context matching students' interests \\
\cline { 2 - 3 } & $\mathrm{S}$ & Student-generated questioning \\
\cline { 2 - 3 } $\begin{array}{l}\text { Giving } \\
\text { encouragement, } \\
\text { assistance, and } \\
\text { feedback }\end{array}$ & $\mathrm{Se}$ & Differentiated learning \\
\cline { 2 - 3 } & $\mathrm{H}$ & Self, involving personal identities \\
\cline { 2 - 3 } & $\mathrm{Fe}$ & Feed-forward academic \\
\cline { 2 - 3 } & $\mathrm{A}$ & Feed-back academic \\
\cline { 2 - 3 } & $\mathrm{Pr}$ & Praise \\
\hline
\end{tabular}

The analysis of the audio-recordings indicated that the interactions between SS and WK and their students were predominantly focused on mathematical content and learning. Their lessons started promptly and finished by revisiting the lesson's learning and signalling connections with future work. Throughout their lessons, frequent links were made to prior knowledge, and greater use was made of teacher questioning in whole class teaching sections than that of the less caring teachers. Specific praise was used judiciously. The audio-recordings of these teachers' lessons sounded vibrant, teachers and students appeared to enjoy the lessons, be comfortable to use opportunities for reflecting their personal identities, and laughter was present.

The teacher-student interactions (whole class and teacher-individual) of UU and ST were often frequently repeated, yet comparatively ineffectual, procedural and behavioural instructions not directly linked to mathematical content and learning:

Quickly people, sitting down, books, out. (ST)

Keep the noise level down please. (ST)

I want everyone paying attention please. I'm still waiting for some people. (UU)

In UU's and ST's classrooms potentially rich mathematical tasks when present tended to be undermined by poor communication of the purpose of the task. Lessons typically took 10 to 15 minutes to get underway and tailed off without a definite end, again with more focus on procedural issues (e.g., 'chairs up', 'books away') and including little or no consolidation of new work or links to future work. 
Links to prior knowledge were less strategic, less frequent, and less clearly stated and expectations of work level and output were low. Students were praised for the quantity or quality of their work although, in the researcher's opinion, the work and/or effort were poor. The lessons lacked academic challenge and students appeared to find them boring, frustrating, and unsatisfying. Laughter was infrequent and when it occurred it tended to be at the expense of other students or the teacher.

\subsection{Caring relationships in action: An example of a lesson exhibiting teacher care}

The following description is of one lesson given by teacher WK. In order to provide a "rich thick description" of findings (Creswell, 2003, p. 196; Patton, 2002, p. 437) supplementary examples from WK's other observed lessons are used to more fully illustrate and discuss aspects of his typically caring teacher practice. Of the ninetysix lessons observed for this study, this lesson was the one most closely exhibiting 'mālie' (Manu'atu, 2000, Section 1.2). This section describes an example of a caring lesson and links to the study model through illustrating a possible lesson within the caring mathematics whare tapa wha, an example of the best practice possible when appropriate conditions exist. The lesson is described in terms of planning and preparation, reinforcing entry into and exit from the community of practice, teaching strategies and teacher behaviours, and enriching the learning community.

Before the lesson the teacher had attended to marking, lesson planning and readying the classroom. The students had completed a Numeracy assessment task for national qualifications during the previous mathematics lesson. These tests had been marked and annotated with specific feedback to be returned to the students.

Detailed planning was evident for this lesson. Teacher prepared handouts included: information on horsepower and the horsepower ratings of cars; a practical investigation to measure students' horsepower values for walking and running up steps; and instructions regarding presentation of the investigation results. The lesson included a variety of activity styles: teacher exposition; individual work (student reading and use of formulae); a group activity to collect data (completed outside); use of measurement equipment (measuring tapes and stop watches); and working in pairs to carry out calculations and prepare a poster of their work. Opportunities for enhancing all sides of the tapa wha were present, for example through: 
- an engaging context to develop mathematical understanding and display new learning (taha hinengaro);

- an investigation carried out by the whole class working in small groups outside the classroom, teacher participation, and making posters for the classroom (taha whānau);

- opportunities for one-to-one interactions, humour, and learning about each other as individuals, and developing personal mathematical identities (taha wairua); and

- moving around and out of the classroom, the physical aspects of the outdoor investigation such as collecting and using measuring equipment, walking, and running (taha tinana).

The classroom was ready for the students' arrival, the whiteboard displaying a message congratulating them on their Numeracy assessment results (Figure 5.7) and the lesson starter activity (daily practice questions). The classroom was clean and tidy and the teacher was waiting at the door to welcome the students.

Congratulations! $9 / 19$ or $47 \%$ of the class have gained Numeracy level 1 .

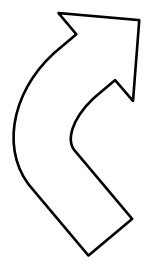

Malo le taumafai! ${ }^{28}$

Tino pai! ${ }^{29}$

This could potentially be $12 / 19=63 \%$ if three students can answer one question correctly.

Figure 5.7: Blackboard message for students arriving into class

WK used consistent daily practices to begin and close his lessons that helped to reinforce the community of practice of the classroom. Because the classroom was ready for the lesson, WK was able to greet students by name as they arrived, initiate quick conversations with students who were early, re-establish his relationships with students, and re-orient them to their mathematics learning:

Hats off, [non-regulation] shoes off, thank you for coming!

28 'Congratulations!'

29 'Very good!' 
This lesson began in a positive way through teacher and student-initiated greetings and WK congratulating the class on their learning. Students late to class spontaneously apologised for their lateness as they arrived in the room. Their apologies were expected; verbally accepted by WK and did not appear to interfere with other students' learning. WK reminded students of his expectations when necessary:

[One late student on arrival:] Volleyball meeting, Mister.

WK: That's not the way to come into my room Thomas.

Student: I'm sorry I'm late sir.

WK: Apology accepted Thomas.

As students worked on the review questions, WK congratulated them individually on their Numeracy results, acknowledging publicly several students who achieved excellent results, and checked that the students had the equipment and scrap paper they needed for the lesson. He quickly circulated the room to check each student's homework had been done. As a result of this style of lesson beginning, WK had interacted one-to-one with every student several times within the first five to ten minutes of the lesson.

At the end of each lesson WK used a routine of students standing behind their desks and listening while he summed up the lesson, praised them for the day's work, and as he released them, expressed good wishes for the rest of their day or weekend. At the end of this lesson WK again praised students for their Numeracy assessment results, gave a summary of the key ideas in the lesson, and forewarned students of what to expect in the next lesson. He released them from the lesson with:

Have a great weekend and may the force be with you!

WK's teaching strategies and teaching behaviours helped maximise the students' on-task time and mathematical learning in this lesson. WK prepared students for the new learning through his choice of the review questions used at the start of the lesson (for example by reactivating prior learning of substitution of values into formulae). Other teaching strategies used included:

- incorporating a group task that required at least two people (a time keeper and a runner);

- setting clear expectations of task completion and behaviour outside; 
- including a physical demonstration (WK modelled one horsepower by lifting a chair to simulate lifting $15000 \mathrm{~kg}$ over a distance of $30 \mathrm{~cm}$ over a time of 1 minute);

- providing a choice of activity for those who preferred not to go outside (none took this opportunity);

- checking for understanding of language, content, and the task during teacher exposition; and

- repetition (e.g., repeatedly using the same formula for different calculations).

In all lessons WK kept students on task through using signals (e.g., warning of how much more listening time there would be before getting on with the task), setting an urgent pace, using focused pauses/wait time to pull individuals back on task, varying the use of his voice (pitch, pace, volume, tone), and interacting with every student multiple times (e.g., asking and answering questions, roving).

Typically WK enriched the students' sense of learning community by incorporating praise, using and managing humour (teacher and student-initiated), and displaying personal engagement and investment in the lesson content, the students, and their progress. This lesson included all of these. WK took many opportunities to praise students' efforts and progress:

[roving looking at students' books] I like what I see, people!

Karina, I hear you did really well in basketball yesterday, well done!

In this lesson WK used humour ${ }^{30}$ (also see Section 9.1.4) in several ways:

- to help construct the classroom atmosphere:

I'm not picking on you Barry because I've picked on you twice today. I'll pick on Chris because I haven't picked on him today. "You have!" "Yes, I know I have!"

You are late Regan! You've obviously slept in and you look so much more handsome for all that extra sleep!

- to maintain students' focus on the lesson content; and

Don't go to sleep Rachael, please. I know this is exciting!

I could just say that those who have done Ex 1.11 and 1.13 get to go to lunch!

I'm giving you this one because I know you just love factors of 42 .

\footnotetext{
${ }^{30}$ Whilst some of the examples of WK's humour quoted here could be interpreted by some to be sarcastic in nature, it is the researcher/observer's opinion that sarcasm was neither the teacher's intent nor the students' interpretations of these comments. The comments appeared to be kindly meant and interpreted with good humour.
} 
- to diffuse situations and for classroom management:

Come on guys, can you help Regan find his shoe? You know how much he cries when he hasn't got his shoe!

WK involved himself personally in the lesson in three substantive ways. He started the discussion about horsepower by explaining his own interest in cars and their horsepower ratings, he participated in the physical task walking and running up steps and encouraging others as they walked and ran, and made the worksheet for the class outside class time. Students' confidence in WK's commitment to them and their learning is illustrated by student-initiated interactions and students' engagement in set tasks. Students spontaneously asked questions when they needed assistance or wanted to know something. WK encouraged this by always responding with complete answers. Further, he facilitated informed student decisionmaking, for example, by giving matter of fact explanations regarding possible repercussions of students' decisions, e.g.,

Being late makes it harder to know the work well enough to get school credits. ${ }^{31}$ You don't' show your working, you don't get the credits. You don't get the credits; you don't get into Year 11.

The students enjoyed and respected their teacher and enjoyed their mathematics lessons. These factors facilitate the success of any lesson. While this section discussed one caring lesson, it must be seen in the context of the teaching and relationships developed by WK with this class over time. Thinking, doing mathematical tasks, success in learning, smiles, and laughter, all were evident. Students were able to interact with mathematics, the teacher, and one another, and were fully engaged. They were able to use physical, mental, and creative dimensions of their personality in carrying out the lesson's learning experiences. A sense of energy and vitality could be felt during the lesson.

\subsection{Care for students as culturally located individuals}

This section discusses the use of culturally linked learning experiences and teacher behaviours in the observed lessons.

No mathematics learning experiences were observed using contexts which drew from the expertise, knowledge, or interests of students' heritage cultures. SS expressed that a lack of suitable resources was one of the factors limiting her use of

31 The Year 9 and 10 levels of this school use a rewards system with school credits given to students for good work, good work habits, and extracurricular successes. For students to have access to Year 11 classes they must acquire a specified number of credits. 
such activities. Although subsequently provided with suitable resources the teacher did not incorporate them into her teaching. UU reported success with student motivation and culture-related discussions following her use of similar resources with a class not part of the study. Although UU agreed to provide student feedback on these activities and was provided with teacher and student feedback forms, no feedback was received.

SS respectfully acknowledged to students in her first lesson with them that many in the class spoke languages she did not have the ability to speak. However, she did not refer to this again or draw on the students' language skills. There were only very occasional examples of two or three study teachers using a word or two of Māori or a Pacific language across all observed lessons. However, not all one-to-one conversations could be heard during the lesson observations or in the audio recordings and it may be that examples of teachers acknowledging students' cultural expertise and knowledge were missed.

Limited opportunity to trial the section of the observation schedule focused on teacher care of students as culturally located individuals is a likely contributor to the lack of data recorded in this area. While this section of the schedule was informed by literature and consultation, few opportunities to refine it arose through the classroom trials. Use of the schedule during the study highlighted one aspect as particularly difficult to observe (i.e., understands and allows for students' code/s of behaviour and cultural responsibilities). Knowledge regarding teacher decisionmaking would be required to record data for this item and would have been difficult or impossible to gather during the observations themselves. However, the observation schedule was only one of the data gathering tools used during the lessons. Although culturally related learning experiences, behaviours, and use of te reo Māori or Pasifika languages could also be recorded on the audio-recordings and in field notes, little such data emerged.

Four of the study teachers were very experienced in teaching Māori and Pasifika students in New Zealand classroom settings. Furthermore, one had over ten years' experience teaching in schools in Pacific Nations, and another was of Māori heritage. Despite their combined experience with Māori and Pasifika students and their knowledge of these cultures, very few data were collected across all lesson observations within the section of the schedule for recording teacher care for students as culturally located individuals (Appendix 3). This result is somewhat baffling and more research is needed to explore why teachers are not making more 
use of their and their students' culturally located expertise and knowledge in their teaching.

There may be several reasons for study teachers choosing not to use learning experiences or teacher behaviours linked to heritage cultures:

- data may have been missed;

- the heavy time and energy demands of the teaching profession; lessons outside normal teacher practice taking more time to plan and develop;

- lack of teacher knowledge of students' cultural heritages or lack of pedagogical knowledge of how to reflect these in their teaching;

- teachers perceiving mathematics to be culture-free;

- the lack of a whole school focus on acknowledgement and incorporation of students' cultural heritages; and

- the enculturation of teachers and students into traditionally 'mainstream' education.

The substantial enculturation of teachers and students into the traditional schooling system is likely to be a key reason for the lack of reflection of students' cultural heritages in mathematics lessons. Furthermore, there may exist an unstated understanding that the cultures most influential to students at school are schoolrelated (the school group, year group, their class, and their friends) and that these cultures take precedence in the school setting over heritage-based cultural knowledge and expertise. The low student uptake of questionnaires in te reo Māori and Samoan, despite the numbers of students who indicated being able to use these languages, ${ }^{32}$ may also be indicators that students are more comfortable at school fitting in with the traditionally dominant culture. Evidence emerged through the study that some students and teachers believe that students would feel 'shamed' or 'singled out' if such knowledge was reflected in lessons. For example, according to several Pasifika study students and the Pasifika cultural advisor, some Pasifika students are embarrassed at their lack of proficiency with their heritage language and cultural knowledge. It may be that teachers consciously or unconsciously acknowledge these areas of discomfort by treading a safe path with all students in

\footnotetext{
${ }^{32}$ Some level of te reo Māori was spoke in at least five study students' homes and one questionnaire was completed in te reo Māori. Some level of at least sixteen students' heritage Pasifika language was spoken in their homes and three questionnaires were completed in Samoan (Section 8.1.1).
} 
perpetuating a traditional classroom style that reflects the historically dominant culture. This complex area is further explored in Chapters Six and Eight.

\subsection{Discussion}

In general, the lessons exhibiting the most caring teacher behaviours and practices were those that held the greatest student engagement (i.e., highest levels of on-task student behaviour) and the most student-initiated interactions (related and unrelated to mathematics). Students of the most caring teachers appeared confident they could access assistance when needed and aware that their teachers persevered with their learning as a class and as individuals. Teacher perseverance appeared to encourage student perseverance.

The students of the less caring teachers were more likely to exhibit off-task, disruptive, unresponsive, and challenging behaviour, and more negative body language (such as turning away from the teacher). These students tended to take much less care with learning and bookwork than those of the more caring teachers. 


\section{CHAPTER SIX}

\section{Teachers' Perceptions of their Practice}

This chapter presents the analysis of, and findings from, the data collected from the twenty-four teacher interviews and eighteen teacher questionnaires. Teachers' views regarding the value of teacher-student relationships (Section 6.1), how they establish and maintain rapport (Section 6.2), and the place of cultural identity in mathematics teaching and learning (Section 6.3) are presented. Because effective teacher-student relationships and mathematical learning were consistently present in SS's, WW's, WK's, and UM's lesson observations, greater emphasis is placed on their data than data from teachers UU and ST.

Teachers' questionnaire data were analysed manually. The data from the interviews and questionnaires were coded and categorised using both "investigative" (or "inductive") and "interpretive" (or "deductive") methods (Onwuegbuzie \& Teddlie, 2003, p. 370; Patton, 2002, p. 453) (Section 3.5). A matrix of dispositional and culturally responsive themes (Figure 6.1) was developed to provide a framework for analysing and discussing the results. The matrix forms the woven whāriki (floor mat) of the teacher care mathematics whare model (Section 3.5.1, Chapter Nine) illustrating the fundamental importance of the components of firm positive interpersonal teacher-student relationships for classroom learning.

The terms warp (whenu) and weft (aho) are used to describe the two sets of threads used in weaving. The warp describes the lengthwise threads that provide the basis for weaving the weft and the weft is used to create the patterns. For this whāriki, the threads that form the warp are drawn from a framework for culturally responsive teaching (Averill, Te Maro et al., 2009):

- Knowing each other;

- Knowing each other as learners;

- Knowing each other's cultures; and

- Enhancing feelings of cultural identity.

Interpretive methods were used to explore the data using the themes constituting the warp of the whāriki.

The dispositional themes, built on the themes of culturally responsive teaching of the warp, form the weft of the mat. Investigative methods were used to determine the 
dispositional themes by arranging the data into categories until saturation was achieved and no further categories found (Chapter Three). The themes were:

- Liking each other;

- Respecting each other;

- Being tolerant of each other; and

- Being able to reflect personal identity.

The matrix of themes, the whāriki, is used to help present the analysis in Sections $6.2,6.3,7.1$ and 7.2 .

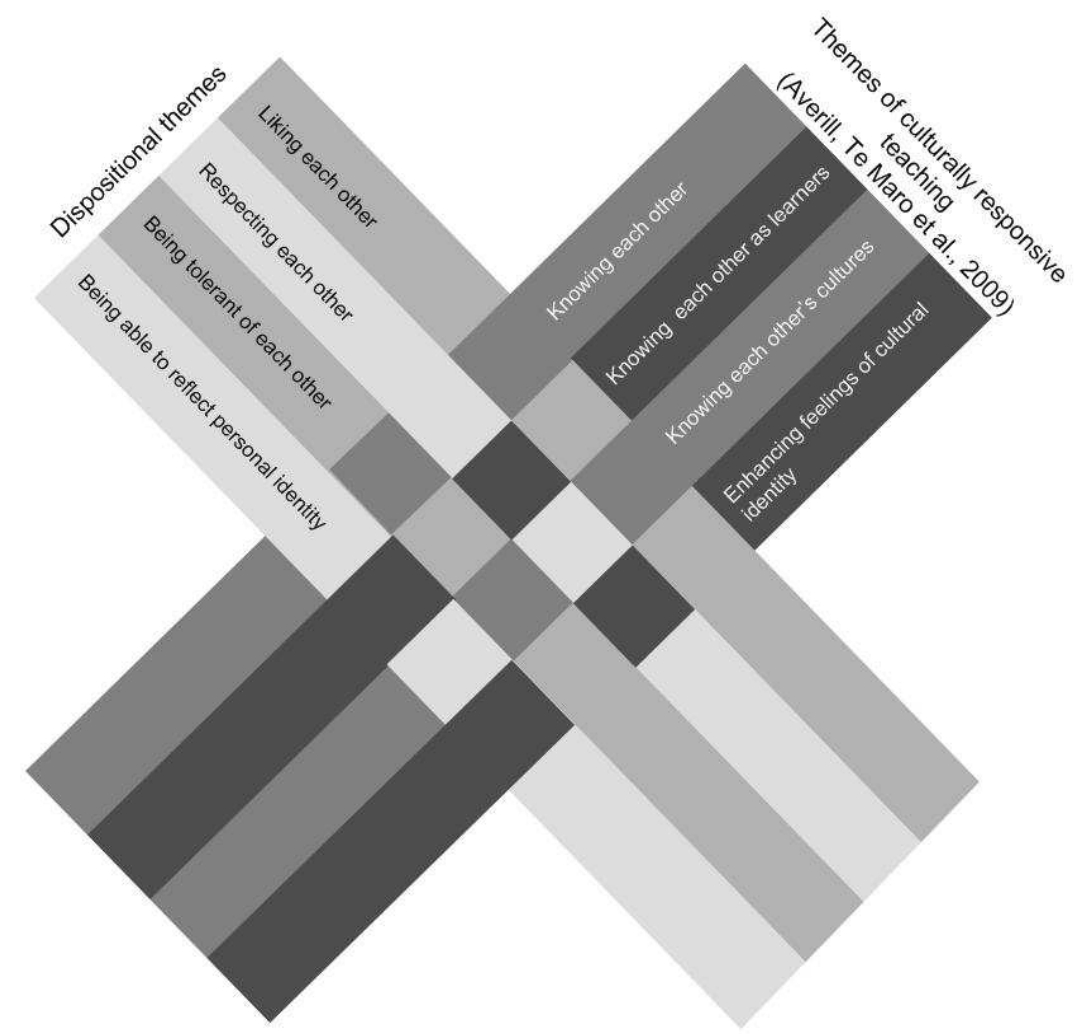

Figure 6.1: Matrix of coding themes

\subsection{Teacher-student relationships - teachers' views}

All teachers were emphatic that effective teacher-student relationships are important for student learning, shown by the strength of language in their responses (e.g., 'critical', 'essential', 'vital', 'number 1', 'extremely important'). Teachers reported that strong relationships promoted engagement with the teacher and the subject, and increased their own enjoyment of teaching the class:

How important do you believe good teacher-student relationships are for the students' maths learning? (Interviewer) 
Critical. Literally critical. If you don't have good student-teacher relationships then there's no way to learn and it's as simple as that. (WK)

I think good [relationships] are essential for all learning, it doesn't matter whether its maths or whatever subject. If you don't have a good relationship with the kids then it impacts on your ability to teach and their ability to learn. It's something that has to be developed over time. There are other things that are important but if you don't have a relationship you can't teach. (WW)

Well, for me, relationships are really number 1 . If students don't relate to their teacher then they don't relate well to the subject either, so it's very important. (UU)

I think they're extremely important, especially at year nine, year ten levels where students are still relatively immature and what is more important to them is how they feel in the classroom environment as opposed to how well they should do academically. For the good students who are obviously keen to do well, it is nice for them to have a good working relationship with their teachers. And if you go down to the other end for the potentially difficult students, it helps a lot as far as classroom management goes. You need to have a reasonably good rapport with them as opposed to starting off on the wrong foot and then having that confrontational thing straightaway, then every time when they come to a class, all they can think about is, I still have my thing, you know, I still have to sort it out with the teacher before I'm happy to learn. So it is very important to make sure that you have a reasonably good working relationship with all the students in your classroom. (ST)

I think it's vital. If you get along with them, from my experience, they will listen and listening is the most important part of what we are doing. If they are not listening, you can't teach them. They won't listen to somebody who they don't like. (UM)

Having personal relationships, being able to say 'how are you?', 'what are you doing?'... that it makes it a pleasure.... It's easier to teach. (SS)

\subsection{Establishing and maintaining rapport}

This section describes the teachers' views regarding establishing and maintaining rapport with their students within four broad areas: teachers and students knowing each other as people, teachers knowing their students as learners, the strategies teachers use to establish and maintain rapport and the challenges to doing so, and the student characteristics teachers believe support and hinder rapport building.

The teachers described how they demonstrated respect, courteousness, and care for their students as individuals, and made opportunities for students to speak in class in order to create classroom environments conducive for them and the students to get to know each other:

At the start [of the year] I maintain some distance from students. I show students respect and I'm polite. I try to make students feel worthwhile. (WK)

For me it's shutting up and watching in the first instance and being prepared to say sorry and apologise in the right places at the right time and to let them 
know that we [teachers] do things wrong as well. I'm as honest as I can be with them. (WW)

Even though I know them all I might just go around and get them to introduce themselves again so they have a chance to speak in the class straightaway. (SS)

The study teachers believe that knowing the students as people and being open to students knowing them are useful for developing rapport:

How important do you feel it is for you to know about your students as individuals? (Interviewer)

It's useful in terms of interacting with them, and also for them to know about us as individuals, little bits and pieces about us. These kids are very inquisitive in that sense. Some staff think of that as being rude or nosy but the kids often want to know about the people around them, where you come from, who your wife is, what kids you've got and that sort of stuff. And for them, they are always pleased when we show an interest in something that they're doing. For us [asking questions] is a bit different, if you start asking direct questions they may think you are being nosy. Knowing about each other just helps. At times it can help you through an awkward situation in teaching. (WW)

Examples of teachers expressing their own personality as a way to establish or maintain rapport included deliberate sharing of information about themselves and their ideas and using their humour, as shown by the following:

It's a two way street because I often used to pack them up ten minutes early and it would be question time and they would put their pens down, and then I would say 'right, you can ask me questions now, because you don't know much about me' and so you would get your usual questions, like 'how old are you?' and then as they got past that, the questions got deeper and more meaningful. Then you pick up more from them because they would end up saying 'hey, that's how we do it at our house.' Fundamentally I think you've just got to be an all round nice guy and if you can do that and show an interest in the kids, it's good. (WK)

It seemed to me with the first lesson with the class that you used quite a bit of humour. Is that a way that you use to establish rapport? (Interviewer)

Yes, especially with those academic classes. If it's not an academic class, I'm not using so much humour. They don't like that always, but sometimes it works quite well l've found. It is my way, to use humour by being a bit cynical, you know? It's part of my nature and my personality. (UM)

So you being yourself in the classroom is one of the ways that you establish relationships? (Interviewer)

Yes, otherwise l'm being fake. I'm not an actor, you know. (UM)

Ways in which teachers get to know their students range from deliberately formal methods to informal methods. All but one of the teachers (ST) prioritised learning students' names. Two teachers (SS, UU) asked their students to write letters about 
themselves at the start of the year and SS deliberately shared information about herself with the class before setting the letter writing task:

I always tell them something about myself, how long l've been teaching, my children, that kind of thing, and if they want to ask me anything now's the time. They don't generally ask anything too personal. Always on the first night for homework I get them to write me a letter just to tell me everything that they think I should know. I have particular themes: how their last year went; what they want to do this year; their family; and anything I need to know - you know like if they've got a job or they don't feel well, or there's something that they think the teacher should know. It's quite motivating and always they write really good letters and tell me lots of things about themselves (SS).

On the whole the teachers reported favouring informal methods of the class and themselves getting to know each other as people, such as informal conversations with students over time (SS, WW, UM) and contact with families (WW).

I get to know students through talking with individuals about things other than maths including asking personal questions, when appropriate. (WW)

I think really I would just like to know about the students by having more time to talk with them, asking them questions, talking in small group situations is better. (SS)

I try to find out a bit about them, about their personal life and I can utilise that, for example I know some people that smoke and then I can go have a quiet whisper in their ear about smoking and it builds up rapport in regard to discipline in the class. You can find out what they are interested in and what their hobbies are. (WK)

In general teachers stated that they found out about their students directly from each student. One teacher also expressed wanting students to be comfortable in sharing information about one another, as appropriate:

l'd also like it if there was more of a culture of looking after your friends, so that other kids who knew, for example, that there was a problem, could say so-and-so is not feeling very happy today, or whatever. (SS)

Teachers reported liking to know a range of information about their students (Questionnaire One, early Term One), including students':

- 'successes' (all teachers);

I do know it's good if they've won something, or done really well in something outside of school, or even inside of school, or in another subject, to acknowledge that. It's always handy to know it, because you can congratulate them about it. I'm not sure how l'd like to find these things out. It would be good if the school could tell you but we don't always get that information. (SS)

- 'sports', 'personalities' (5 teachers each);

- 'families', 'family commitments', 'cultural activities' (4 each); and

- 'progress in other subjects' (3 teachers). 
In Terms Two and Three teachers reported knowing more about students' 'personalities' and 'successes' than any of the other characteristics. WW's and WK's data demonstrated the biggest increases in knowledge about their students over the year and only one teacher (ST) indicated no growth in knowledge about their students.

All teachers believed in the importance of incorporating aspects relevant to the students' school and out-of-school lives in their teaching with most reporting they do so (in-school experiences: ST, WW, UU, UM, and out-of-school experiences: ST, WW, WK, UU, UM). Their reported rationale for doing so included to help show the relevance of the learning (UU) and to motivate students (SS, WW). The examples of incorporating aspects from the students' lives into their mathematics teaching given by teachers were general in nature rather than linked to specific knowledge of individual students, e.g., using budgeting, shopping, and sports statistics:

\footnotetext{
I try to use as many stories that they can relate to as possible to explain new concepts. This involves bringing in students' experiences that I know of and what l've learnt from students. (WK)
}

I write a lot of problems that link to things going on in the school. (UU)

None of the examples given by teachers relate directly to students' cultural backgrounds or ethnicities. Furthermore, mathematics learning experiences carried out during lesson observations were not drawn from teachers' knowledge of students as individuals and were infrequently linked to students' school or out-ofschool experiences (also see Section 5.2.3). Two teachers (SS, ST) stated it was harder to relate learning to students' lives in mathematics than in other subjects they had taught with SS stating that she did not incorporate such aspects but she felt that she should.

Teachers were not asked how they used their knowledge of students as individuals in other teaching decisions and it is possible that this information is used to guide pedagogical decision-making such as grouping of students, modes of interaction, and expectations.

Strategies used by the teachers to get to know the students as learners included the use of diagnostic testing (SS), brainstorming when introducing new learning (UM), working one-to-one, and speaking with as many students as possible each lesson (SS). Teachers reported a range of ways that helped their students know them as the teacher including establishing a worthwhile programme (WK), having an 
organised environment (WK), encouraging students, having patience, and listening (WW), and setting clear boundaries, routines, and expectations (WW, WK):

At the start of the year I'll say welcome back to my room. I always say 'my room.' This is not their room; this is my environment. I set it up. They do have a hand in it once they have shown me they are responsible. I'll outline the routines to them; no chewing in class, no graffiti in books or desks, no jackets on inside. You all know the rules, revision is up on the board, go for it and that will be about it. So there's no namby-pamby stuff. I just get into it. (WK)

When asked to describe the strategies they used to maintain rapport with students (Questionnaire Two, Appendix 8), teachers reported using methods that would help develop their and their students' knowledge of one another as people and as teacher/learners. Those likely to help develop the teacher and students' knowledge of one another included environmental factors (e.g., making sure students know what is happening (WW), being flexible and fair (WW), creating a friendly environment (UM)) and personal elements (e.g using a sense of humour (SS) and talking with students on a daily basis about their life, their progress, out-of-school activities, and problems (WK)). Teachers' ways of supporting and caring for students likely to develop knowledge of one another as teacher/learners included helping students be successful (UM), taking care not to embarrass students (UM), and helping students in lessons of subjects other than mathematics (WK).

Teachers' opinions regarding effective means for establishing and maintaining rapport were probed using strategies students believed to be important, as gathered from students in Term One (Questionnaire One, Appendix 7). From most important to least important, the teachers' responses to the student-identified strategies were:

- 'knowing students' names' and 'using praise' (all teachers strongly agreed with both);

- 'listening to students', 'showing they like teaching the class', 'using humour' and 'being patient' (four or five teachers strongly agreed with each);

- 'smiling', 'rewarding good work', 'having fun learning maths', 'giving students' choices', 'using correct Māori and Pasifika pronunciation' (most teachers agreed or strongly agreed); and

- 'setting harder work' (three teachers agreed).

These results indicate that similarities exist between students' and teachers' views regarding effective strategies for rapport building (Section 7.1.1). 
Teachers' thinking about the student characteristics that affect teacher-student rapport was explored. This was done by asking teachers in all questionnaires to name three students with whom they felt strong rapport and three with whom they felt weak rapport along with the main reasons for their choices. Student characteristics linked to teacher-student rapport reported by teachers related to knowing and liking the students as individuals (responsiveness to the teacher, personality, disposition, and previous knowledge of one another) and as learners (disposition towards learning, learning needs, and work habits) (Table 6.1).

Table 6.1: Teacher perceptions of student characteristics contributing to teacher-student rapport

\begin{tabular}{|c|c|}
\hline $\begin{array}{l}\text { Student characteristics with positive } \\
\text { effects on teacher-student rapport }\end{array}$ & $\begin{array}{l}\text { Student characteristics with negative } \\
\text { effects on teacher-student rapport }\end{array}$ \\
\hline \multicolumn{2}{|c|}{ Knowing and liking the student as an individual } \\
\hline $\begin{array}{l}\text { Responsiveness } \\
\text { responsive to personal support (WK), easy } \\
\text { to get along with (WW), wants to know me } \\
\text { (SS) }\end{array}$ & $\begin{array}{l}\text { Responsiveness } \\
\text { uncommunicative and unresponsive to me } \\
\text { (SS, ST, WW, WK) }\end{array}$ \\
\hline $\begin{array}{l}\text { Personality } \\
\text { sense of humour (ST, WW, WK), likeable } \\
\text { (WK), open (SS), friendly (ST, UM), good } \\
\text { manners (WK, UM), needing personal } \\
\text { support (WK), confident in expressing } \\
\text { opinions (WW) }\end{array}$ & $\begin{array}{l}\text { Personality } \\
\quad-\end{array}$ \\
\hline $\begin{array}{l}\text { Disposition } \\
\text { positive (SS), willing (WK, UM) }\end{array}$ & $\begin{array}{l}\text { Disposition } \\
\text { negative attitudes towards the teacher (ST, } \\
\text { WW, WK, UU, UM) } \\
\text { negative attitudes to school policies (e.g., } \\
\text { uniform, lateness, homework) (SS, ST, UM) } \\
\text { poor relationships with other students, } \\
\text { confrontational (ST) }\end{array}$ \\
\hline $\begin{array}{l}\text { Previous knowledge of one another } \\
\text { taught the student previously (ST, WK, UM), } \\
\text { previously taught or met some of the } \\
\text { student's family (SS) }\end{array}$ & $\begin{array}{l}\text { Previous knowledge of one another } \\
\text { not knowing the student well (due to student } \\
\text { being new, self-sufficient, shy, or } \\
\text { independent) (SS, ST, WW, WK) }\end{array}$ \\
\hline \multicolumn{2}{|c|}{ Knowing and liking the student as a learner } \\
\hline $\begin{array}{l}\text { Disposition towards learning } \\
\text { initiative in asking questions about the } \\
\text { learning (SS, WW, UM) }\end{array}$ & $\begin{array}{l}\text { Disposition towards learning } \\
\text { lack of initiative for learning that increases } \\
\text { the teacher's workload (e.g., dealing with } \\
\text { absences, lateness, providing constant } \\
\text { supervision) (SS, WK, UM), negative } \\
\text { attitudes to mathematics (SS, WK, UM) }\end{array}$ \\
\hline $\begin{array}{l}\text { Learning needs } \\
\text { needing mathematics learning support } \\
\text { (WW) }\end{array}$ & $\begin{array}{l}\text { Learning needs } \\
\text { good at mathematics and therefore does } \\
\text { not need or seek help (SS) }\end{array}$ \\
\hline \multicolumn{2}{|c|}{ Respecting the student as a learner } \\
\hline $\begin{array}{l}\text { Work habits } \\
\text { work output (SS, ST, WW, WK, UM) } \\
\text { improving achievement (WK) }\end{array}$ & $\begin{array}{l}\text { Work habits } \\
\text { students who do not try (SS, WK, UM) }\end{array}$ \\
\hline
\end{tabular}


Teachers' questionnaire responses indicated that they found students' personalities, work habits, and having prior knowledge of the students to be most important for high levels of teacher-student rapport. In contrast, aspects most likely to undermine teacher-student rapport were students' negative dispositions towards the teacher, school, and mathematics learning, the teacher not having prior knowledge of the student, and poor student work habits.

Challenges teachers reported facing in establishing rapport included holding conflicting roles (i.e., deaning ${ }^{33}$ vs teaching), students' prior negative school experiences (e.g., students arriving with poor work habits (WK, UM)), lack of time for one-to-one interaction (SS, WW) sometimes due to large class sizes (ST, UU), outside influences, and being out of touch with students' lives outside school:

The out-of-school influences on students and my 'old age.' (WK)

SS and WW reported finding a balance between the discipline-related deaning responsibilities and nurturing aspects of their teaching roles difficult at times.

Most study teachers (SS, ST, WW, WK) felt that mathematics teachers faced greater challenges than teachers of other subjects in developing teacher-student rapport. They argued that in mathematics lessons it can be more difficult to interest students in the content, that missed lessons are more problematic in mathematics than in other subjects due to the sequential nature of mathematics learning (WK), national assessment methods are reducing how interesting students find mathematics, and that many students arrive with negative experiences of, and feelings towards, learning mathematics (SS, WK):

Do you find that there's any difference in terms of developing rapport with the class, in teaching Maths than in teaching other subjects? (Interviewer)

Yes, I think it can be harder. I found when I was an English teacher, kids could find something that they love and wish they could read, even if they're not a good reader. If you put out lots of magazines and lots of newspapers, there's always something that they can find of interest to read. Also because I'm a reader, I used to enjoy reading to them and talking about books, that kind of thing. It's easier to make [English] exciting and interesting. You can watch a film; you can do a play. There's that wide variety of things. It's not as much chalk and talk [as mathematics teaching is]. And I think kids find that even though there's lots of exciting things in maths, [external assessment] drives us so that kids and parents and teachers are so focused on passing that you can't afford the time to do the interesting enjoyable things like patterns in Geometry, where you colour them in, spirals and all that kind of thing. The whole emphasis of assessment going down to third and fourth form means that all of the joy's going out of maths. (SS)

33 In many New Zealand schools, a 'dean' oversees each year level group of students. The dean's role often involves pastoral care and disciplinary components. 
Often [maths teachers] have to work harder [at developing relationships]. Kids' relationships with you are affected by their view of the subject quite often and if they don't feel switched on by the subject it's probably going to be difficult to get a good relationship with them. So somehow we've got to get them to come in and feel comfortable, so being successful in maths to start with is important, even if it's just real simple stuff. That means on the first test [I make sure] everyone gets more than eighty percent, so that they feel good about it. (WW)

All teachers felt that it was not only essential for establishing and maintaining rapport to know about their students as learners and to know individuals' mathematics progress, but it was also vital to care, and show that they care, for their students' mathematics progress. They believe that caring for students' learning results in motivational effects on both their teaching and the students' learning. In the teachers' view, students are more likely to care about their learning if the teacher does, and conversely, they are less likely to care about their mathematics learning if they feel their teacher does not care:

Caring about their progress helps my motivation. (WK)

If I didn't care about their maths learning, what is the point of doing the job which is to make a difference, to help students improve their self confidence, achievement levels, and to enjoy maths. (WW)

A lot of the students' belief that what they are doing is worthwhile is through teacher expectation. If you don't care, they know it and they don't like it. (SS)

[Caring for their learning] helps the students feel wanted and able to achieve. They will want to try harder. (WK)

Teacher care for students' progress was explored by examining the goals teachers had for the study classes at the beginning of the year, all of which indicated their care for students' learning. The goals related to:

- students' progress with the course content (to improve students' problem solving skills (WW), for students to achieve level 1 Numeracy and be ready for NCEA mathematics ${ }^{34}(\mathrm{WK})$, for them to move up one or two [curriculum] levels (ST), and to ensure students can make sense of their answers (UM));

- pedagogy (to incorporate differentiated learning (SS)); and

- students' attitudes to mathematics (to have students being positive about mathematics (WK) and believing in their ability to learn and understand mathematics (UU)).

${ }^{34}$ Level 1 Numeracy and NCEA refer to the Year 11 level of New Zealand's national qualification for secondary school students. (See Section 1.1) 
The main ways teachers reported they show that they care about their students' mathematics progress were by: knowing, acknowledging, encouraging, and praising individuals' progress (all teachers); being available to provide assistance in and out of class time (all teachers); being enthusiastic about the subject (WK); and acknowledging (with students) factors which may affect students' progress (WK). Specific examples included:

- highlighting students' achievements, being positive about progress, giving regular feedback (WW, WK), praising good work efforts (SS, WK, UM), rewarding good work with less formal lessons (WK);

- talking about targets, the importance of doing well, and doing homework (SS, WK), constantly encouraging students to do better, "accepting them where they are but encouraging them to improve" (WW);

- frequent one-to-one interactions (SS, WW, UM); and

- trying to link home and school expectations (SS).

All teachers reported giving students oral feedback often and written feedback seldom. The oral feedback was described as informal and was given as teachers assisted students working independently or in groups. Three teachers commented that lack of time constrained the amount of written feedback they provided to students (WK, UU, and UM). The low emphasis on written feedback, prevalence of informal feedback, and teachers' perceived time constraints on giving feedback do not match well with students' feedback preferences (Section 7.2.2) and are likely contributors to students' lack of confidence that their teacher knows how well they can do mathematics. Ways to improve the quality and quantity of feedback on individual students' learning is a useful area for further exploration.

Two teachers (WW, WK) reported students asking them often about their progress and four (SS, ST, UU, UM) that students sometimes asked about progress:

I like them to ask questions, tell me how they found the homework and discuss their aims. (SS)

Overall the study teachers believed that knowing their students as individuals and as learners, caring for students' mathematics learning, and establishing respectful teacher-student interactions are important for developing and maintaining rapport. Some elements of teacher behaviour were reported to assist in developing both knowledge of students as people and as learners (e.g., one-to-one interactions, teacher questioning) and others were more suitable for developing one or other 
(e.g., acknowledging students' sporting successes, giving specific feedback on learning). In questionnaire responses regarding how teachers establish or maintain teacher-student rapport, no mention was made of students' cultures and ethnicities.

This section has shown the importance placed by the study teachers on establishing and maintaining rapport with their classes and has provided insights regarding strategies teachers can use to develop the dispositional and culturally responsive themes of the whāriki (Figure 6.1). The results indicate the importance for mathematics learning of deliberate and visible teacher emphasis on developing classroom relationships by showing care for students and for their mathematics learning.

\subsection{Cultural identity and learning mathematics - teachers' views}

This section describes teachers' views regarding the relevance of their own and students' cultural identities to students' learning. Teachers' own cultural identities, the difficulties they had responding to questions from this area of the study, and how they reflect students' cultural identities in their mathematics teaching are presented.

Three teachers identified themselves as New Zealand European (WK, WW, UU), one as New Zealand European/Māori (SS), one as Asian (ST), and one as East European (UM). ST and UM both speak their heritage language fluently, others speak only English. As aspects special to their cultures, teachers listed: education (WK, ST); personal responsibility (ST); cultural acceptance (SS); politeness (WK); and respect for others (WK). Three teachers did not provide responses to this question, one reply being:

I find it hard to identify anything special about European culture - it is easier for those of other cultures (e.g., Samoan, Chinese) to do this. (WW)

The significance for teachers of their heritage culture and that of their students was explored in a range of ways through all data gathering instruments. Teachers generally had greater difficulty in responding to questions regarding their own and students' cultural identities than to other questions. The teachers' confusion regarding the relevance of students' heritage cultures to mathematics learning is illustrated by responses to different but similar questions giving differing pictures of teachers' views, for example the somewhat conflicting comments from WK taken from different parts of the same interview:

I don't put much importance on, oh what do I say? I put importance, but not in terms of their culture, I mean when they come into the room they're just a group of kids, because I don't care what culture you're talking about, kids are 
kids whether you like it or not, they all want to have fun, they all want a laugh, they all occasionally have to cry and they want to be fair and they just want to be happy so the objective really is to try and get them to be in a happy environment and one that they feel all comfortable coming to, one that they feel is welcoming and that's what I do with, try to do within the frame of the school system... (WK)

I think it's also important to recognise the culture of each kid and try and develop that with the framework of the classroom, not necessarily with the mathematics concepts but because you consider the whole individual rather than just the maths. If you captured the whole individual then hopefully you'll be able to push them. For example I make special efforts to try and learn some Samoan and I have learnt some Fijian words and some Māori words, so if the kids want something, I can speak only a couple of lines, not much at all, but you show that you're making the effort. Kids just love it, you get it all wrong and it's something they can teach you and so it makes them feel important and it makes them feel important because you are respecting their culture and their heritage and so in that way, yes, I think it is very important that you recognise each kid as an individual. (WK)

Teachers' responses to the questions related to caring for students as culturally located individuals varied more between teachers than those given for the questions focused on caring for students as individuals and as mathematics learners.

Teachers were asked to indicate the importance placed on students' cultures by their school (Questionnaire Three). They indicated that students' cultures were:

- $\quad$ very important (WW, WK) or important (SS, ST) to their school (examples given included having a marae (traditional Māori meeting place) at the school (WK), school-based cultural groups (SS, WK) and cultural events (SS), ethnic mix of student leaders (SS), expectation on staff for correct pronunciation of names (WW), and of finding out about students' cultures (WW)); or

- not important to their school (UU) (reasons included that "students' cultures were not mentioned in administration or pastoral meetings," Māori students having excellent pastoral care within the school, and support for school staff on Māori-related issues (from the Māori teacher) but no similar support regarding issues related to other cultures).

Teacher UM did not know the importance placed by his school on students' cultures.

Results obtained when teachers were asked the importance of a range of personal, work-related, and community groups they were involved in for their life in general and their mathematics teaching (Questionnaire Three, Appendix 9) showed teachers ranked their heritage culture in the top five groups for importance both in their lives and their mathematics teaching (Table 6.2, Figure 6.2). 
Table 6.2: Importance to teachers of the groups they belong to

Note: Teachers indicated the relative importance to them of each group using 0,1 , 2 , or 3 check marks. Overall scores for each group were calculated by adding together the total number of check marks for each group.

\begin{tabular}{|c|c|c|}
\hline $\begin{array}{l}\text { Rank } \\
\text { order }\end{array}$ & $\begin{array}{l}\text { Importance to my life: } \\
\text { (total \# check marks on diagram - five } \\
\text { respondents - WW did not complete } \\
\text { the diagram) }\end{array}$ & $\begin{array}{l}\text { Importance to me when I am teaching } \\
\text { maths: } \\
\text { (total \# check marks on diagram - six } \\
\text { respondents) }\end{array}$ \\
\hline $1^{\text {st }}$ & My family (15 points) & This class (16 points) \\
\hline $2^{\text {nd }}$ & My department (12) & My department (15) \\
\hline $3^{\text {rd }}$ & This class (10) & My heritage culture, my ethnicity (9) \\
\hline $4^{\text {th }}$ & The staff (9) & The staff (9) \\
\hline $5^{\text {th }}$ & My heritage culture, my ethnicity (8) & My clubs and other interests (8) \\
\hline $6^{\text {th }}$ & My clubs and other interests (8) & NZ community (7) \\
\hline $7^{\text {th }}$ & NZ community (7) & My family (6) \\
\hline $8^{\text {th }}$ & My church group (6) & Local community (6) \\
\hline $9^{\text {th }}$ & Local community (5) & My church group (2) \\
\hline $10^{\text {th }}$ & My sports teams ( 1 ) & My sports teams (0) \\
\hline
\end{tabular}

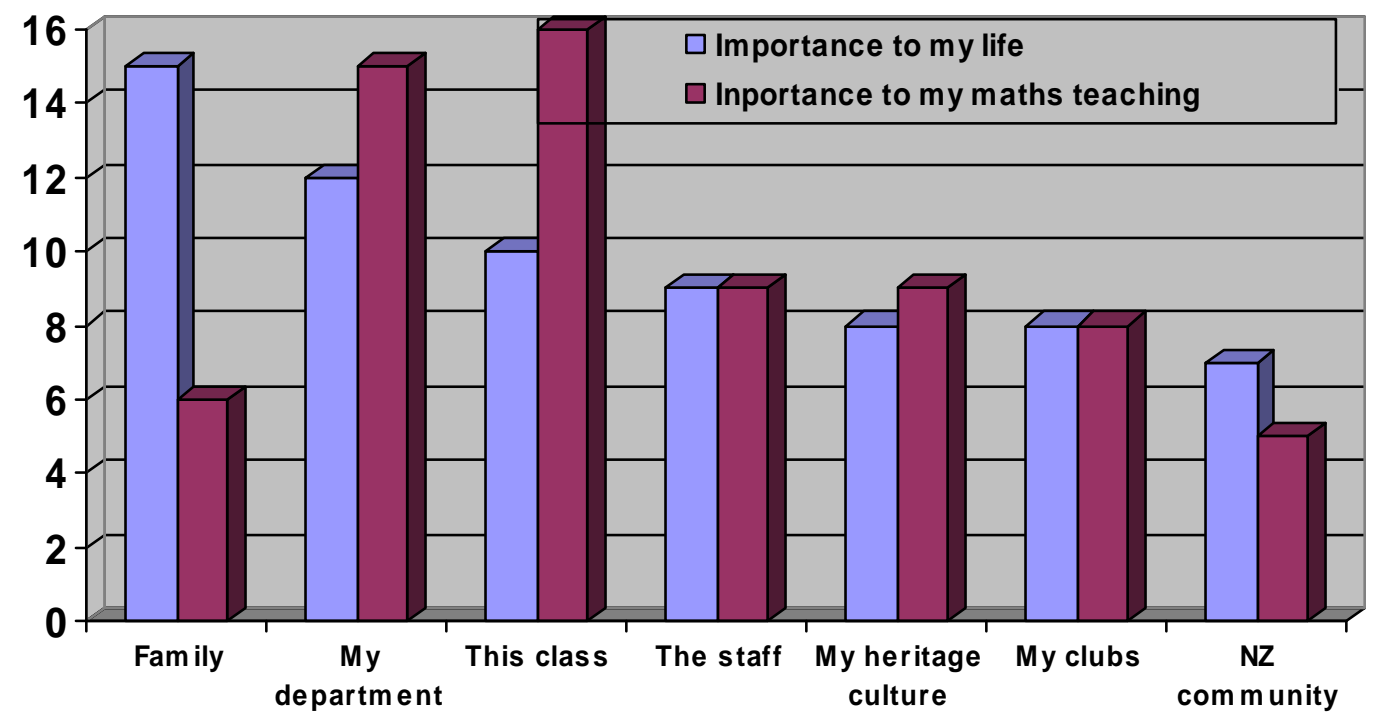

Figure 6.2: Importance to teachers of the groups they belong to Note: The figure includes the seven groups with the highest frequencies.

However, teachers' responses to a further question in the same questionnaire indicated they considered heritage cultures (teachers' and students') less relevant for mathematics learning than is suggested by the data in Table 6.2. In this question, three teachers (SS, WW, WK) reported their 'ethnicity/culture' was relevant when at school and teaching mathematics. Others (ST, UU, UM), reported being neutral to both.

The study teachers regard students' cultures as more relevant than their own cultures both to their work and their relationships with students. Questionnaire 
responses indicated that students' cultures are important to all teachers, particularly WW, WK, and UM. All teachers indicated that they like to know about students' cultural identities with WW and WK expressing this more strongly than others. All reported knowing some, most, or all of their students' cultural identities and some or most of their cultural activities. SS described why she feels students' cultures are important to their learning:

How important do you feel it is to know about your students' cultural identities? (Interviewer)

Well I think that's important at this school because it's predominantly Polynesian, that's very apparent at this school and they're very comfortable. Having said that if you've got a Māori identity here it's a lot less obvious. There are kids who do have Māori backgrounds who I don't necessarily know about in my class. And then there'd be some who are Asian who may have a different kind of religion and that information is not necessarily given so all of those things, apart from that the Polynesian kids are very much defined by [their heritage groups], some are Tongan and some are Tokelauan and they know that but you don't necessarily know it for each individual. (SS)

I guess if they want you to know it they put it in that letter. (Interviewer)

Yes, they do and also they tell you if it is important, like if you have to ring parents. There's one boy in the $4^{\text {th }}$ form who is Tongan and one of the teachers speaks Tongan so I try to get him to speak to the father who does speak English but not so well. So the more you know their cultural identity, things like that help. Often a lot of our teachers are reluctant to ring parents because their English is a second language that makes the conversations difficult but there's another teacher that speaks fluent Samoan so all those things help. (SS)

Do you think the students want you to care about their cultural identities? (Interviewer)

Absolutely! Gosh, if they think you're insulting them in any way they just have a little hissy-fit and if they perceive a teacher to be racist and for very strange reasons they'll perceive that, they'll get a real snitch on the teacher. For me I do have the benefit of having a Māori background, which always comes up the kids always ask me. And it does make a difference to them. I don't think that they would necessarily say it made a difference to them if you spoke to them but definitely they wouldn't want to be in a school where the staff was all pure white Anglo Saxon in a predominantly Polynesian culture. Having said that students often act in this school in a way that would not be acceptable at home. Their parents were brought up in a different way to them and I don't really think they have a forum to talk about that. Lots of them will poke fun at being belted, like 'you can't tell my parents or l'll get a hiding'. And I say 'look, if that's really true then it's ok to say it but if it is a throw away comment, you can't do that, you can't perpetuate this myth that everybody's parents belt them'. I'm sure some do but there are parents who wouldn't. And it's important they are proud of their culture. Lots of them are bilingual kids. They've got a skill I haven't got but they don't necessarily see it with pride. (SS)

Four teachers (SS, WW, WK, UU) reported that it was important for students' mathematics learning that they not only knew, but that they cared about students' cultures: 
Cultural respect is a must for all teachers. (SS)

It shows students that you value their background; it is all part of sharing an interest in each other so that the learning environment is one that all cultures can work in. (WK)

How important do you feel it is to know about your students' cultural identities? (Interviewer)

I've worked in the Pacific for twelve years; we were in the Cook Islands for eight years, and Nauru, Tokelau, and Samoa. What we've brought back here is knowledge that helps, for example, with some of the new kids. You look at their faces and look at their names, and I said to one of the kids a couple of years ago, 'you're a Cook Islander' and she said 'how do you know?' And I told her the story about a student that I had in the Cook Islands in 1973 that had the same surname and I said this girl came from Mangaia, one of the outer islands and so does she, so it's from those kinds of clues that you can establish some link. A boy I knew was Tokelauan for similar reasons so I said which atoll do you come from and he looked at me and I said the names of the atolls. He said the atoll he came from and asked 'how do you know?' and I said 'well, I lived there for two years'. He hadn't been there because he was New Zealand born but the connections are still there. The game has to be about caring for kids and showing a bit of interest in them. It's more than just the maths. (WW)

Do you think it makes a difference to those kids in terms of their learning? (Interviewer)

It can do. Well I haven't noticed that is really that significant. There are other things that affect their learning more, but it's just that they're more likely to talk to you more, and feel you don't judge things, like when I first came to this school I took everything personally, quite often with what they were saying and how they reacted, but knowing a bit about them and where they come from and understanding that a little bit you know that something they say or do is not meant personally. So it just helps that relationship. You tend not to judge things personally and then you're less likely to get their backs up. (WW)

Teacher UM did not think it important for students' mathematics learning that he care about their cultures and Teacher ST did not know.

In a further question, four teachers (SS, ST, WW, WK) indicated that they felt it important to their class that they care about students' cultures; UU and UM expressed feeling neutral. Examples of how teachers stated that they show their students that students' cultures are important included acknowledging and respecting differences and developing their own cultural knowledge and skills:

Respecting cultural difference. (SS, WK)

Including students' heritage languages into everyday use. (WK)

Talking with students about similarities and differences between our cultures. (WK) 
Giving students opportunities to work in Māori (WW) and attempting to learn te reo Māori. (WW)

Anything that helps with rapport, which is culturally important, I will try to learn about. (ST)

It is important to me to relate to students as individuals. Many go to great lengths to keep their lives in compartments (teachers and class, peers, family and cultural beliefs), for example lots have "school" names (in English) and I hear other students using their Samoan names so I try to know their real names. (UU)

Given that many of their students were of Māori or Pasifika heritage, teachers were asked whether or not they wanted to know about Māori and Pacific worlds. Although study teachers showed interest in knowing about Māori and Pacific worlds, they were largely neutral regarding using mathematics examples linked to using such knowledge in their teaching (Table 6.3). These results suggest that the study teachers perceived classroom mathematics to be separate from Māori and Pacific worlds and that, in their practice, they portrayed this view of mathematics to their students.

Table 6.3: Knowing about and using Māori and Pasifika examples in mathematics

\begin{tabular}{|c|c|c|c|c|c|}
\hline $\begin{array}{l}\text { Questionnaire } \\
\text { statement }\end{array}$ & $\begin{array}{l}\text { Strongly } \\
\text { Agree }\end{array}$ & Agree & Neutral & Disagree & $\begin{array}{l}\text { Strongly } \\
\text { Disagree }\end{array}$ \\
\hline $\begin{array}{l}\text { I like to know about } \\
\text { the Māori world }\end{array}$ & WW, WK & ST & $\begin{array}{l}\text { SS, UU, } \\
\text { UM }\end{array}$ & & \\
\hline $\begin{array}{l}\text { I like to know about } \\
\text { Pacific Nations }\end{array}$ & WW, WK & ST & $\begin{array}{l}\text { SS, UU, } \\
\text { UM }\end{array}$ & & \\
\hline $\begin{array}{l}\text { I like to use Māori } \\
\text { examples in maths }\end{array}$ & WW & UU & $\begin{array}{l}\text { SS, ST, } \\
\text { WK }\end{array}$ & & UM \\
\hline $\begin{array}{l}\text { I like to use Pacific } \\
\text { Nations examples in } \\
\text { maths }\end{array}$ & WW & UU & $\begin{array}{l}\text { SS, ST, } \\
\text { WK }\end{array}$ & & UM \\
\hline $\begin{array}{l}\text { I know about the } \\
\text { Māori world }\end{array}$ & & $\begin{array}{l}\text { SS, WW, } \\
\text { WK, UU }\end{array}$ & ST & & UM \\
\hline $\begin{array}{l}\text { I know about Pacific } \\
\text { Nations }\end{array}$ & & $\begin{array}{l}\text { SS, ST, } \\
\text { WW, WK, } \\
\text { UU }\end{array}$ & UM & & \\
\hline $\begin{array}{l}\text { I use Māori } \\
\text { examples in maths }\end{array}$ & & & $\begin{array}{l}\text { SS, ST, } \\
\text { WW, UU }\end{array}$ & WK & UM \\
\hline $\begin{array}{l}\text { I use Pacific Nations } \\
\text { examples in maths }\end{array}$ & & & $\begin{array}{l}\text { SS, ST, } \\
\text { WW, UU }\end{array}$ & WK & UM \\
\hline
\end{tabular}

All teachers expressed finding difficulty including Māori or Pasifika contexts in their teaching due to:

- lack of resources (SS, ST, WW), "textbooks only pay lip service to [incorporating Māori and Pasifika cultures]" (WW); 
- lack of personal knowledge (WW, WK, UM), "I don't know enough about other cultures and how they use maths" (WK), "identifying realistic situations is difficult" (WW);

I'm not good at this, that's for sure. Overall I'm not making a difference between Pākehā or Māori or something, it's the same, it is in our school. I can't see a difference between them. I'm not sure how I can help somebody who's from another culture to learn maths better. (UM)

- teachers' views regarding students' personal engagement with their heritage cultures (WW), "most of the students are New Zealand born and urban so their contexts are different to those born outside of New Zealand and/or rural" (WW); and

- concerns about possible student discomfort (ST), "students might take it the wrong way and dislike it" (ST).

Two teachers (UU and UM) stated they do not aim to reflect Māori and Pasifika contexts in their lessons. They gave no reasons for this.

The study teachers generally had difficulty responding to the interview questions regarding the professional standard relating to the Treaty of Waitangi (Ministry of Education, 1998) and how they acknowledge the Treaty in their mathematics teaching. However, WW had clearly given much thought to this issue and highlighted factors that impact on teachers' ability to address the Treaty in mathematics classrooms (echoed in WK's and SS's responses), including: a lack of homogeneity of Māori students, a lack of relevance of traditional Māori contexts, lack of appropriate resources, and consistencies between aspects of Māori worldview and those of other groups:

Does the school have expectations in terms of how teachers show evidence towards meeting professional standards about the Treaty of Waitangi? (Interviewer)

That's one of the hard ones. Because we have roughly $70 \%$ Māori meeting those expectations really relates more to the day-to-day dealing with the kids in terms of pronouncing their names and being aware of Māori protocol. And I think for us, there's a huge emphasis at the moment on Māori achievement but most of us actually take it out of that context and look at the bigger picture. It's the achievement of everybody no matter what ethnic group they are. So it's being aware of everybody's background, for all of the kids. In fact when you look at what they expect for Māori achievement, it's no different to anybody else. So I always find this a tricky one, but as far as where meeting the Treaty of Waitangi is concerned, I don't think there's anyone on the staff who wouldn't meet that professional standard at some point because of our day to day interactions with the kids. The most difficult thing is isolating it when you're delivering your curriculum, in terms of contextualising it... (WW)

How you reflect it in a maths classroom. (Interviewer) 
Yeah, the day we actually get effective resources to do it is the day then that I feel we're able to do our job. And they kind of forget that a lot of our kids are urban Māori, they're not rural. (WW)

It's a different issue again? (Interviewer)

It is. And so I think there's actually huge bold assumptions made about the kids' connections with their tipuna (ancestors). And most of these kids don't have them, or don't realise that they're there, they haven't got the connections. And so the assumptions are that if we put things in [traditional Māori] contexts, that it's going to do all marvellous stuff, but it doesn't. It's a much bigger problem that that. If you're going to look at education for Māori, you're always told the Māori world's a holistic one - I figure that's actually no different to anybody else because when kids grow up they don't compartmentalise anything, it's when they get to school that it's compartmentalised. So whatever you do for Māori should be what you do for everybody in that sense. And so when you talk about contextualising - well, I wrote a geometry unit in Māori when I was doing study, but all it was, was mathematics written in Māori with a few Māori examples. And that was really just window-dressing, it was paying lip service. My intentions were good but it's not what it's about. Not the way I see things. (WW)

\subsection{Discussion}

Given that the teachers' preferred method of getting to know their students is through informal opportunistic conversations it is likely that the most extrovert students and those most used to sharing personal information spontaneously are known most well by their teachers. There are also likely to be cultural as well as personality factors at play leading teachers to know some students better than others.

While teachers did not seem as surprised as their students to be asked questions linking heritage cultures and mathematics learning, they generally appeared not to have developed a firm philosophical stance nor to have deeply considered how they would or could demonstrate cultural responsiveness in their classroom. Whilst many textbooks do not yet incorporate cultural contexts in any substantial way, such resources have been available for some time (e.g., Averill et al., 2003; Heays, Copson \& Mahon, 1994; McMurchy-Pilkington, 1996; New Zealand Association of Mathematics Teachers, 2001).

That teachers and students (see Chapter Eight) seemed somewhat confused regarding questions relating to cultural identity and comparatively weak in answering these questions could indicate that discussions focused on catering for cultural diversity are not taking place in their school or department or are not seen as important by their school or their school community. The rather sketchy consideration and description of links between mathematics learning and culture 
seem consistent with the level of cultural responsiveness within their wider school environments (Chapter Four).

In general, teachers' responses portrayed some confusion about the relevance of their own and their students' heritage cultures to mathematics learning and a lack of commitment to reflecting students' cultures in their teaching decisions. This is in stark contrast to their comfortable and confident responses regarding the importance for students' learning, of knowing and understanding their students as individuals and as learners. 


\section{CHAPTER SEVEN}

\section{Students' Perceptions of Caring Mathematics Teachers}

This chapter presents the analysis of the data collected from the student interviews and questionnaires regarding teacher care for students as individuals and for students' mathematics learning. Analysis of the student responses relating to students' cultural identity and culturally responsive teaching are reported in Chapter Eight.

The ninety-three student interviews were analysed using the whāriki matrix of dispositional and culturally responsive themes (Figure 6.1). Questionnaire data were analysed using SPSS. Section 7.1 describes the results using the dispositional themes (liking each other, respecting each other, being tolerant of each other, and being able to reflect personal identity). Section 7.2 presents analysis organised using two of the culturally responsive themes (knowing each other, knowing each other as learners). Section 7.3 provides a summary and discussion of the analysis results.

Students' questionnaire responses have been combined across all study classes, and to expose differences across ethnicities in the results, they are identified by students' ethnicity where possible. Two types of student ethnicity have been used in the analysis: students' self reported ethnicity and that reported to the Ministry of Education (see Section 4.4). Self-reported ethnicities are only available for the students who completed Questionnaire One (roughly $70 \%$ of student participants overall) whereas students' ethnicities recorded using the Ministry of Education guidelines are available for all named students' questionnaires and most interview data. To best explore the data using the students' perspectives of their ethnicities, the figures and values in this and the next chapter use the student data grouped by students' self reported ethnicities. In order to maximise the questionnaire data that could be linked to students' ethnicities, many other results in this and the next chapter are identified as the ethnicities provided to the Ministry of Education. For clarity in this and the next chapter regarding which type of data have been used:

- Māori ${ }^{\star}\left(\mathrm{M}^{\star}\right)$, Pasifika* $\left(\mathrm{P}^{\star}\right)$, New Zealand European* $\left(\mathrm{E}^{\star}\right)$, indicate student ethnicity as self reported; and

- Māori (M), Pasifika (P), New Zealand European (E), Other (O), indicate student ethnicity as reported to the Ministry of Education.

$\mathrm{N}$ is used to indicate that the student's ethnicity was not recorded. All totals include all student participants. 


\subsection{Dispositional themes}

Each of the dispositional themes from the whāriki matrix (Figure 6.1) will be discussed in turn, using summaries of students' views and examples of how they report their teachers display aspects of the theme. The themes are not mutually exclusive. Where decisions had to be reached regarding placement, the theme most clearly related was chosen.

\subsubsection{Liking each other: "He gets along with everyone, that's one of the good things"}

The majority of students felt it important that the students and teacher like one another in order to be motivated to learn, to achieve in the subject, and to feel confident that the teacher would support them when they needed assistance:

Do you think it is important to have a good relationship with your teacher to learn Maths well? (Interviewer)

Yes, I do, 'cause if you don't really like the teacher you don't want to listen to her or him or do well in your work. (SS student)

Why do you think it's important to get on well with your teacher to learn maths well? (Interviewer)

'Cause you have to get on well with your teacher if you want to get on well with your subject. (WW student)

Do you think it's important for your learning to get on well with the teacher? (Interviewer)

Yes, it is important, 'cause last year, my last maths teacher, we didn't get on very well and it made my learning hard cause I didn't like her. We didn't like each other, so it made it hard for me to learn with her. (SS student)

How important is it for you to get along with your maths teacher? (Interviewer)

It's good to get along with your maths teacher so if they can be there for you when you fall down or if you get behind you can ask for guidance. (WK student)

For students, the teacher and students developing a liking for one another was assisted by their teacher showing knowledge of individuals through:

- showing care for mathematics learning and/or care for the person;

Do you think your teacher cares about who you are as a person? (Interviewer) 
Yes, because, like, if I wasn't at maths yesterday, she'd ask 'are you OK?' to make sure that everything's all right. (SS student)

What are your teacher's main ways of getting on well with the class? (Interviewer)

If people have a problem she listens to them and if they don't get it she'll explain it again....and if people want to do a certain starter she'll let us do that one. (SS student)

- giving time to one-to-one teacher-student interactions;

Do you think your teacher cares about who you are as a person? (Interviewer)

Yes. (SS student)

How do you know that? (Interviewer)

Like the way she is around us, she's not like, just do that, she sits down with you and she talks with you and that gives you a warm vibe. (SS student)

- developing the class learning community;

What are the teacher's main ways for getting on well with the class? (Interviewer)

I think she likes to do icebreakers and stuff at the start, to kind of get people used to the idea of doing maths before we start doing the actual lesson, and I think that helps her get on a bit better because you get kind of warmed up for the lesson. (SS student)

Is there any part of the lesson that you enjoy the most or that is the best part for you? (Interviewer)

For me it's probably the class discussion, 'cause we sort of have fun as well. (SS student)

It's probably the same for me as well. (SS student)

OK, so you like it most when the teacher is up at the board, he's helping with the notes, explaining the new work, and he asks lots of questions and then asks if there are questions. (Interviewer)

Yeah. (UM student)

Yeah, 'cause we're all as a whole group, like the whole class doing it so it's more fun. (UM student)

- using humour:

Are there any difficulties for your teacher getting on well with your class? (Interviewer)

Not really, because he makes us laugh a lot, which is good. (UM student) 
The Māori ${ }^{*}$ and Pasifika* students were more likely than the New Zealand European* students to respond with 'always' regarding whether their mathematics teacher cared about them, wanted to help them learn, and encouraged them to do their best (Figures 7.1-7.3) ${ }^{35}$.

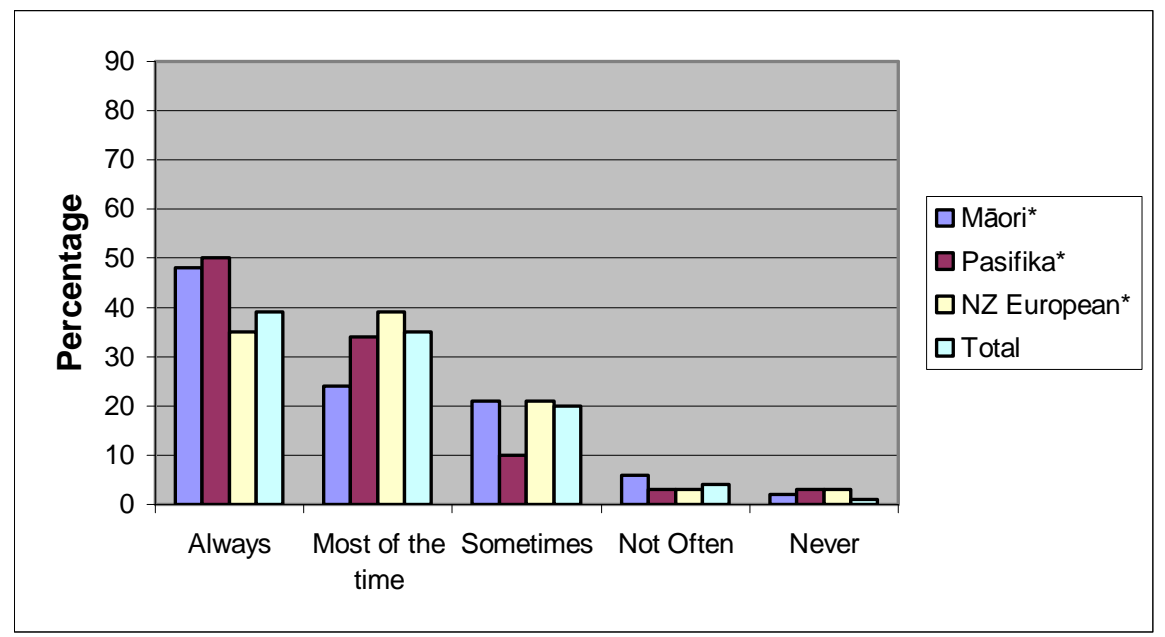

Figure 7.1: Students' responses to 'My teacher cares about me' Note: Mean \% for Questionnaires 2 and 3

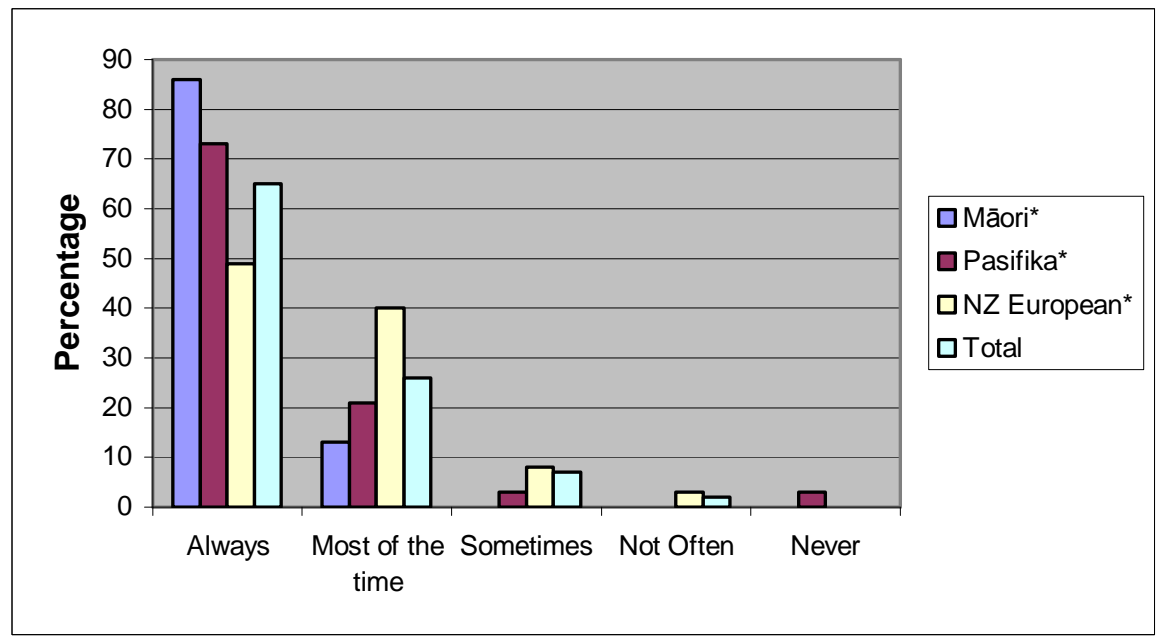

Figure 7.2: Students' responses to 'My maths teacher wants to help me learn' Note: Mean \% for Questionnaires 2 and 3

35 Measures of significance for exploring whether the differences between ethnic groups were significant were not calculated for the data used for these or subsequent figures due to the tests' unsuitability. The following reasons informed this decision: some students were in more than one group; the results were not normally distributed; the data was subjective and qualitative; and the sample sizes were not sufficiently large. 


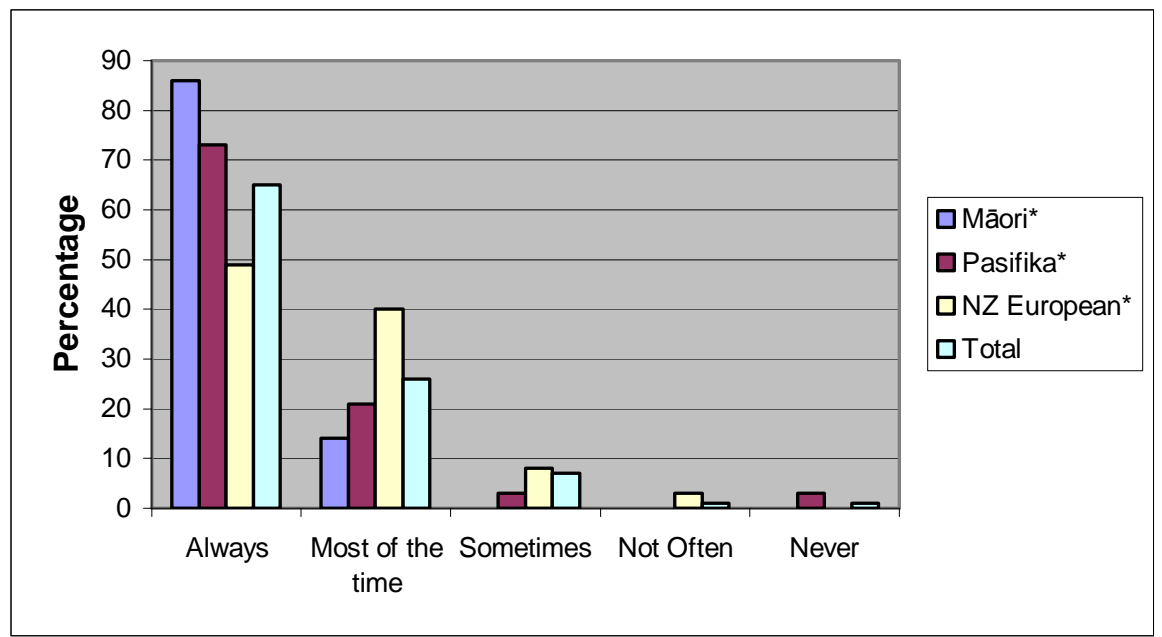

Figure 7.3: Students' responses to 'My teacher encourages me to do my best' Note: Mean \% for Questionnaires 2 and 3

Many students believed that liking the teacher was more important than knowing about them as a person:

Is it important to know a little bit about who your teachers are? (Interviewer)

Not really, just as long as we learn. Because once we leave college we're not going to be really involved with them. (SS student)

I know some teachers tell you all kinds of things about themselves and others tell you almost nothing, so where's the best part of that continuum for a teacher for you guys? (Interviewer)

I don't mind if I don't know about them but as long as I get on with them it's all right. (SS student)

Most students had a clear feeling that their teacher cared for them (Figure 7.1) but in the interviews many had difficulty expressing how they knew this. This was shown in several ways, for example by long pauses before a response, looks of confusion, or responses reiterating that they knew their teacher did care about them but they did not know how they knew this. Others expressed mixed feelings about whether or not their teacher cared about them:

Do you think your teacher cares about who you are as a person? (Interviewer)

Sometimes I don't think he really does...sometimes you just feel that way, you know, but other times he makes you laugh...but it's just sometimes he makes you think he doesn't really care. (UM student)

On the whole, students felt it important for their progress in the subject that they liked their mathematics teacher. Some also wanted to know about their teacher and their teacher to know about them as individuals and as a class so that the teacher could maximise students' learning through understanding their needs and the ways in which they liked learning as a group (see also Section 7.2.1). 


\subsubsection{Respecting each other: "We all respect him because he respects us"}

Students' comments, some explicit and others implicit, showed they feel respect to be an important facet of teacher-student relationships. Showing respect positively affected the nature of one-to-one interactions and helped develop trust. Teachers knowing about students' successes both within and outside the classroom enabled students to feel their skills were acknowledged and affirmed.

So what's different when your class comes to maths from how you are in other subjects? (Interviewer)

I don't know, I guess it's the whole environment of how he treats us. He's really good and we all respect him because he respects us, so that's probably the main thing. (WK student)

What do you think makes a good maths teacher? (Interviewer)

[Having] respect and making maths fun, so people want to do it. (WK student)

What did you put in the questionnaire that you'd like your teacher to know about you? (Interviewer)

My sports and stuff, like if I do well in a test or something, yes, just my successes and kind of like who I am. (UM student)

Students' lack of respect for their teacher created challenges for teacher effectiveness:

Are there any challenges for your teacher getting on well with the class? (Interviewer)

Yes, we're all noisy and don't really listen most of the time, so it's harder for him. (UM student)

Yeah, our class is quite disrespectful towards lot of our teachers. We'll talk and they'll try and get us to be quiet so it ends up the whole class getting detentions because we're not quiet. (UM student)

Teachers can show respect for students in many ways; teaching well, having high expectations, giving positive feedback and encouragement, acknowledging and enabling acknowledgement of work, helping, answering questions, and sharing decision making. The following student comments indicate how these practices affected students' thinking about their learning:

I quite enjoy maths with our teacher. (UM student)

Yeah he's a good teacher. I suppose I did hate maths in primary, but I don't hate it now. (UM student)

Yes, because we're actually understanding it with him. That makes it a lot easier. (UM student) 
How does your teacher encourage you in Maths? (Interviewer)

Oh she always pushes us, and she always makes us answer the questions and stuff on the board, which like puts pressure on you but then if you don't get it she'll explain it to you. (SS student)

Do you think your teacher believes in your ability to be successful in maths? (Interviewer)

Yes, because she's always telling us that. If we don't do so well in the test she says, 'you can do better than that, I know you can', and so you do. (SS student)

So what is it that makes a maths teacher a good maths teacher? (Interviewer)

One that doesn't mind too many questions, 'cause if I ask Mr UM too much he gets a bit annoyed. One that's friendly like my last teacher... she was real nice and she loved me asking questions and she always mentioned me for asking heaps of questions. She used me as an example for the other kids... I felt special. (UM student)

What does your teacher do that helps with getting on well with the class? (Interviewer)

Sometimes I think when we listen to music it just settles us down more. Because we know it's not his responsibility to do our work, it makes us do our work. (WW student)

He's encouraging you to make your own decisions about the work? (Interviewer)

Yeah, he's treating us like equals, not like we're kids. (WW student)

Students indicated that their teachers' interpersonal skills (e.g., knowing about individuals, listening, spending time one-to-one, and encouraging personal responsibility) were important for showing their respect for individuals:

Is it important to you that your teacher shows that they care about who you are as a person? (Interviewer)

I'm not sure, as long as they know who I am and respect that it's not about involving time and spending time as a person. (WK student)

What do you think is the most important thing that we should tell student teachers about being a good high school maths teacher? (Interviewer)

Listen to the students. Help them if they need help. (SS student)

Do you think it is important for your teacher to show they care about people's cultural identities? (Interviewer)

Yes, because that just makes people feel good because they know they are respected. (WK student) 
The students' perceptions of their teacher's respect for them was also explored using the student questionnaires (Figures 7.4 and 7.5).

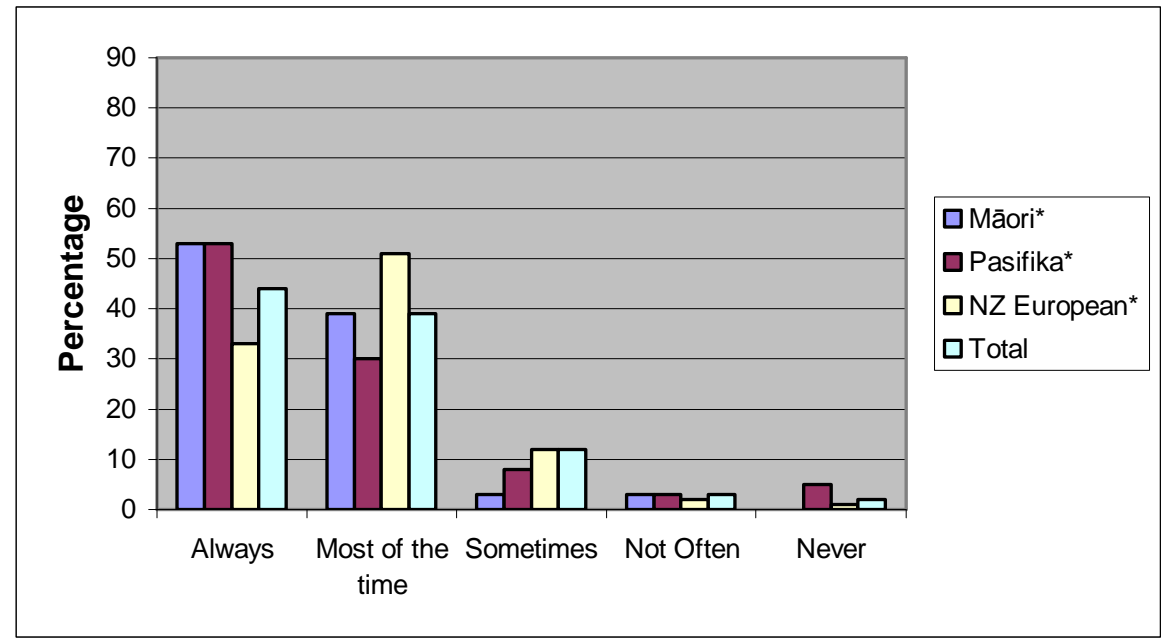

Figure 7.4:

Students' responses to 'I like the way my teacher treats me when I make a mistake' Note: Mean \% for Questionnaires 2 and 3

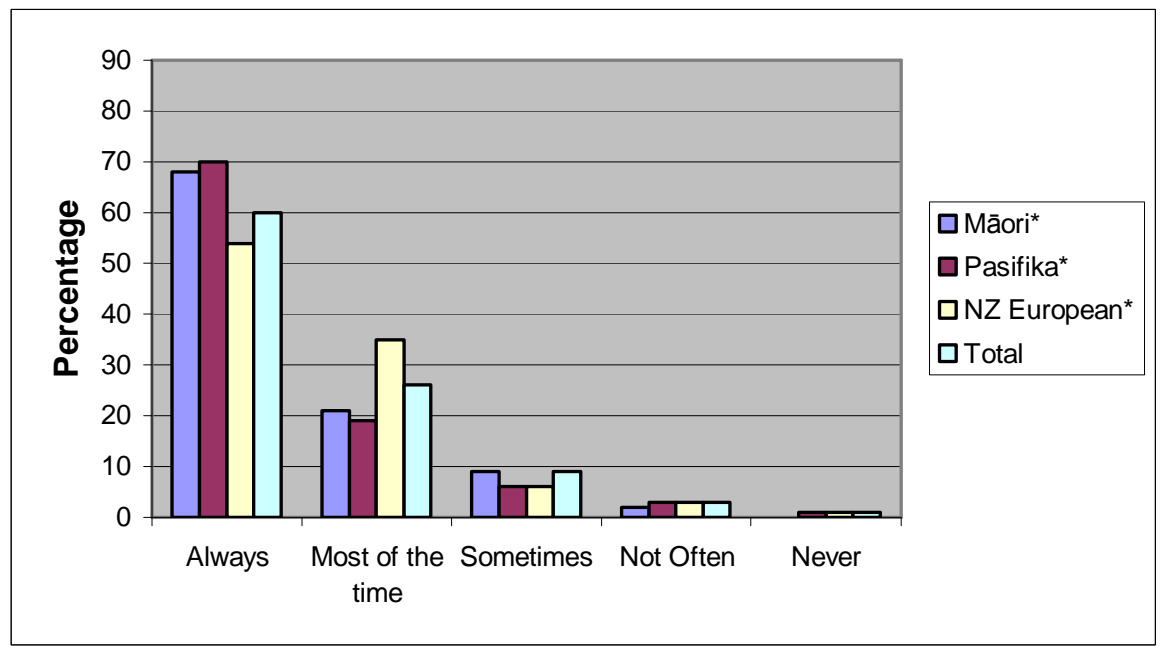

Figure 7.5: Students' responses to 'My teacher treats me with respect' Note: Mean \% for Questionnaires 2 and 3

The responses indicated that the Māori* and Pasifika* students were more likely than their New Zealand European* counterparts to like the way their teacher treated them when they made a mistake (Figure 7.4) and to feel treated with respect (Figure 7.5). Further exploration of how mathematics students develop feelings of teacher respect is necessary to determine possible reasons why these differences have arisen. Possible reasons could include that the Pasifika* students in this study may have been more secure in their own cultural identities than the Māori* and New Zealand European* students and may therefore be less likely to interpret teacher actions as disrespectful. Teacher behaviours could be more in tune with what constitutes 'respect' to Pasifika students. For New Zealand European and Māori students, respect is more likely to have to be earned than directly given in contrast 
to those from many Pasifika cultures for whom respect is often given for knowledge and age. Therefore the Māori and New Zealand European students may arrive in class waiting to see if the teacher will 'earn' or create a respectful relationship with them rather than having an assumption this will be the case.

The giving and receiving of respect between teachers and their students was seen by many students (across ethnicities) as important for their learning. Most study students felt respected by their teachers and most indicated having some level of respect for their teacher. Respect for each other was associated with engagement with learning, teaching approach, teacher manner, and knowing and acknowledging aspects important to them of students' lives (see Chapter Nine).

\subsubsection{Being tolerant of each other: "Maybe there's something that we won't understand and she needs to know that"}

Students value tolerance. Knowing about the students enabled teachers to understand their students and show them tolerance:

Do you think the teacher knowing a little bit more about each person helps with your maths learning or not? (Interviewer)

It could. If the teacher knew something about you personally then maybe when you answer a question or you don't, or you don't do something, it could help them understand why. (UM student)

Yes, that's right, just have a bit more of an understanding is good. (UM student)

Maybe if they knew you had sports team practice, and you're a bit tired, that's the reason why, so it's not just 'cause you're slacking off, it's because...yeah, they'd know. (UM student)

Students stated that teachers showed tolerance for them in the way that they taught, having patience, persevering with help, and knowing students as learners:

What is it that makes a maths teacher a really good maths teacher? (Interviewer)

One that like doesn't mind you asking questions if you don't understand. (UM student)

Yeah and helps you with problems that you don't understand. (UM student)

And they're clear in the way they tell you [about] the work you're supposed to be doing. (UM student)

Anything else that's important? (Interviewer)

They have to be nice. (UM student)

Nice, so can you explain nice a bit? (Interviewer) 
Sort of caring. (UM student)

Patient. (UM student)

And so with the caring, that's caring about who you are as a person or caring about the class? (Interviewer)

Both of them. (UM student)

Do you feel that your teacher encourages you personally or encourages you as a whole class or both? (Interviewer)

Class, both. (UM student)

Probably both. (UM student)

And how does he do that? (Interviewer)

He just helps us a lot, when we're stuck on questions. And then he just, will keep on going through it until we have it right, or he'll do more examples of the work that we're doing if we don't get it. (UM student)

Yeah, he just never gives up, just gives examples till we know it. (UM student)

Students felt that teachers showed tolerance for students in their interpersonal skills, knowing and respecting their mathematics abilities and their cultures, and by treating students like family:

Do you think it's important that the teacher cares about how you feel about your culture? (Interviewer)

Yeah. (SS student)

Yes, because when we're learning maybe there's something that we won't understand and she needs to know that, you know, maybe we weren't brought up to learn that. (SS student)

So what do you think makes a good maths teacher? (Interviewer)

I think a good teacher will care about the class and be helpful and give easy work when we find it too hard. (WK student)

So when you say care about the class, is that care about them as people? (Interviewer)

Yeah, well I would say care as family. (WK student)

Teachers caring for students as family members is consistent with the concept of 'in loco parentis' (Latin for 'in place of the parent'), which refers to the legal responsibility of a person or organisation to take on some of the functions and responsibilities of a parent, allowing them to act in a student's best interests as they see fit. The previous student response and other similar student responses indicate the depth of relationship that some students prefer to have with their teachers and 
the need for a deeper level of personal commitment than has traditionally been considered within a teacher's role.

Students showed their tolerance for their teacher through being prepared to wait while the teacher assisted others:

Which bits of the lessons do you like the most? (Interviewer)

When he comes around and helps [our group], but the bad thing about that is because he does go round to each group there's not enough time for you. (WW student)

The classroom observations and teacher and student interviews indicated that having and expressing tolerance for one another was related to students and teachers knowing, understanding, and having respect for one another. For example, when teachers explained the rationale for their teaching decisions, students were more likely to be accommodating about the effects on them of those decisions. Similarly, when teachers knew about personal factors impacting on students' ability to complete their homework or get to class on time, they showed greater understanding and tolerance towards these students.

The data indicate that tolerance was developed through students and teachers making and using opportunities to interact with each other, one-to-one, in small groups, and whole class situations. Mutual respect, tolerance, and learning-focused environments in which individuals are comfortable to express themselves freely and reflect their own personal identities appear to be closely inter-related.

\subsubsection{Being able to reflect personal identity: "'Cause it was just funny, and everyone was laughing, it was yeah...happy"}

Teachers and students being able to reflect their personal identities was important to the study students because it enabled them to get to know the teacher, feel the teacher was interpreting their interactions appropriately, and experience a climate conducive to developing their confidence and competence in mathematics:

Can you describe a particular maths lesson that you've enjoyed a lot this year? (Interviewer)

Probably when Mr was just talking the whole time about his holiday. (WK student)

So why was that the best? (Interviewer)

'Cause it was just funny and everyone was laughing, it was yeah...happy. (WK student)

What do you think makes a good maths teacher? (Interviewer) 
Personality and that he works with people. (WK student)

And why is that, how does it help your maths learning? (Interviewer)

It makes you get more into it. (WK student)

Teachers need to make us feel comfortable in the class. They should try not to put people on the spot. (ST student)

Students believed that teachers using real life contexts relevant to individuals, setting high expectations, and using mathematical games created environments where they and the students were able to reflect their personal identity:

Teachers need to know the [students], so if you're not getting something, they can use examples from things you like, like if you are into cars they could say something like five cars were in a race and another five joined in, so how many cars altogether are in there in the race now? So they are using something you are interested in. (WK student)

Make the questions achievable, have high expectations for the class, let us work at our own speed but push us to do more. (SS student)

Can you think of the maths lessons you enjoy the most? (Interviewer)

Oh the ones when we play the games, oh the games, 'cause they're fun and they make you laugh, it's more fun than just writing out stuff. (SS student)

The lesson observations indicated that the ways in which teachers managed and taught their classes also helped create climates where they and students could reflect their personal identity. Examples included:

- how the class and groups were organised (e.g. when teachers allowed students to work on their own or with others as they chose and when students worked in student-selected groups). Those in student-selected groups appeared to have greater engagement with one another, with the teacher (when the teacher was helping the group), and their learning than those in teacher-selected groups;

- teachers assisting and talking with students one-to-one;

- using whole class discussion linking mathematics with real-life contexts (e.g., collecting a complete set of cereal cards (probability), school sports (number and measurement));

- using humour (particularly when responses to their humour were welcomed);

- allowing students to listen to music as they work; and

- giving some free class time. 
These impressions were supported by students' questionnaire responses regarding how they learned mathematics well. They stated teachers should:

let us talk when we need to discuss answers.

take us to work outside some of the time.

use a hands-on approach to learning maths.

make us go up to the board and answer questions to help us know how well we're doing and to build up our confidence.

do group work.

not give lots of homework.

not give boring book work always.

The lesson observations indicated that many students became increasingly comfortable being and expressing themselves in class as the year progressed. This was shown by their sharing problems with the teacher and expressing their ideas and humour with the teacher and their class more freely. Students' comments indicated that the increased comfort levels were due in part to the teacher and the class having experienced more time together:

Well I think that she's nicer now because now she knows us better and she's not so strict any more. (SS student)

\subsection{Culturally responsive themes}

\subsubsection{Knowing each other: "I'd just tell them straight up and then they'll understand"}

Many students felt it important that they and the teacher knew one another for effective communication to occur, for mutual trust and teacher-class rapport to be developed, and their learning improved:

Do you think it is important for your teacher to know who you are as a person? (Interviewer)

Yes, because it kind of breaks down the wall, they can relate to you as a person. You normally think its going to be a scary thing talking to your teacher but if you know a bit about them and they know about you, you feel confident in talking with them. (WK student)

What are the main things that are different about your class when you come to maths from when you have your other subjects? (Interviewer)

It's just the teacher really. How they relate to you and how they teach your class impacts on how the behaviour is. If they get to know you, you know you 
can sort of trust that teacher and you know you're going to get a good lesson. But if they just write something on the board and get you to copy it out, you're not really learning anything. (WK student)

Do you know who your teacher is as a person? (Interviewer)

I would say a little bit because sometimes in class, he will tell us to pack up early and will tell us about Fiji and stuff. (WK student)

And is that a good thing? (Interviewer)

Yes because we learn stuff about him. (WK student)

And why is that good? (Interviewer)

Because we get to know him as a friend and more like family instead of just as a teacher. (WK student)

And if there was anything else you would like to know about your teacher, how would you find that out? (Interviewer)

I would say no, [I wouldn't try to find it out] because it might be personal to him. (WK student)

Early in Term 1, students were asked (Questionnaire One) which personal information about themselves they would like their teachers to know. Later in the year (Questionnaires 2 and 3) students were given the same list of aspects about themselves and asked which they believed their teacher knew. The aspects most frequently chosen by students early and later in the year are compared below. Students had differing combinations of relevant aspects from the outset (e.g., not all students were engaged in sport or cultural activities), which should be considered when interpreting the results.

'My successes', 'my personality', and 'how well I learn in other subjects' were the aspects most frequently selected in Term 1, with over $40 \%$ of students in each group $\left(\mathrm{M}^{*}, \mathrm{P}^{*}, \mathrm{E}^{*}\right)$ selecting these. Almost one fifth of students wanted their teacher to know something about their 'family', their 'sports', and their 'cultural activities' (22$30 \%$ of Pasifika* students and $15-20 \%$ of students across other groups) and only $17 \%$ (16 students) did not know what they would like their teachers to know about them. Four students indicated that they did not want their teacher to know anything about them from the given list and three students did not respond to the question.

The results from Terms 2 and 3 included:

- just over half of the students felt their teachers knew something about their 'personality' (65\% of New Zealand European* students, the highest proportion for any group); 
- $40-50 \%$ of students indicated that their teacher knew about their 'successes' (slightly lower than the number of students who indicated this aspect in Term 1);

- $30-40 \%$ believed that their teacher knew something about their 'family' (slightly higher than the number of students who indicated this aspect in Term 1); and

- fewer than $10 \%$ of students overall (about $15 \%$ of Pasifika* students) indicated that their teacher knew something about their 'cultural activities' (slightly lower across all groups than the number of students who indicated this aspect in Term 1).

The questionnaire results show that as the year progressed, over the entire student group, the teachers knew as much or more about the students as the students had initially indicated a desire for. As three of the study teachers (SS, WW, and WK) had pastoral care responsibilities for their study classes (SS and WW as deans and WK as form teacher), these results are likely to be higher than would be the case for teachers without pastoral care roles.

In general the study students wanted to know a little about their teacher as a person:

As a person, I know [my teacher] has got a family and has a boat, he's a good person, I don't know too much about him but just know who he is. (WK student)

Is there anything else you would like to know about him? (Interviewer)

I am not too sure really, as long as you know who they are, you don't need to know everything about them. (WK student)

Two classes had been asked to write letters about themselves for their teachers at the beginning of the year. Many of these students appreciated the opportunity for helping their teacher get to know them as a person:

[Be]cause if you were too shy to just, you know, speak about it in class, or didn't want to tell anyone, you just write down in the letter and she'd just read it. (SS student)

[Writing the letter] was a real good idea because it gave us a chance to, you know, explain, instead of her saying, 'Oh maybe this person's naughty, I should watch out for that'. It's giving us a chance to write what we wanted. (SS student)

Others liked their teachers to get to know them through the teacher asking them questions, having them complete a questionnaire, talking with their previous teachers, or gradually through one-to-one interactions over time. Some felt comfortable telling the teacher what they would like the teacher to know but stated that was harder in mathematics than in other subjects because fewer spontaneous opportunities for linking the work to their personal lives exist: 
How would you like the teacher to find out things about you that you want them to know? (Interviewer)

I'd just tell them straight up and then they'll understand. (WW student)

I reckon that's pretty hard in maths though, 'cause, you just work with numbers, you don't have conversations about reading a book and then you can't talk about applying it to your personal life or something. (WW student)

Overall, students valued both their teachers knowing about them as people and knowing some degree of information about their teacher. They held differing preferences regarding what they wanted their teachers to know about them, how they wanted their teachers to find out this information, and what they wanted to know about their teacher.

\subsubsection{Knowing each other as learners: "students have different ways of learning"}

In this section we examine students' views about the importance of their teachers knowing them as mathematics learners and how well they believe their teachers know them as mathematics learners. Students' understandings of themselves as learners (in particular, their motivations for working hard in mathematics and how they prefer to be given feedback about their progress) are also discussed.

Students felt it important for their learning that their teacher knew and cared about them as learners:

Teachers can know about your maths progress and they can also show that they care about your maths progress, so out of those two, which is the most important thing to have? (Interviewer)

Probably care about the maths progress. (UM student)

Yeah, so that they help you actually get along, like if you're a bit behind. (UM student)

Because if they just know about your maths progress, they might not do something about it. (UM student)

Yeah, they won't, they probably won't. (UM student)

What do you think is the most important thing that we teach student teachers about teaching maths well? (Interviewer)

I don't know really, I don't know, see, I liked it at primary school 'cause my teachers over the years learnt what my style of learning is, 'cause l'm different to a lot of people. Lots of people like sitting in groups and talking and it helps them concentrate. With me I like sitting in quietness. I like sitting in peace and quiet so it's easy for me. So I guess it would just be that I think that they should learn basically that students have different ways of learning and for 
some students it may be easier to sit there in the quiet, like it is for me, and some it's easier for them to listen to music or to talk or something instead of just sitting there silently. (WW student)

In spite of over $60 \%$ of students feeling their teacher gave them feedback about their progress (Figure 7.6), it is concerning that roughly $50 \%$ of students in Term 1 and $40 \%$ over Terms 2 and 3 indicated they did not know if their teacher knew their mathematical capability (Figures 7.7 and 7.8).

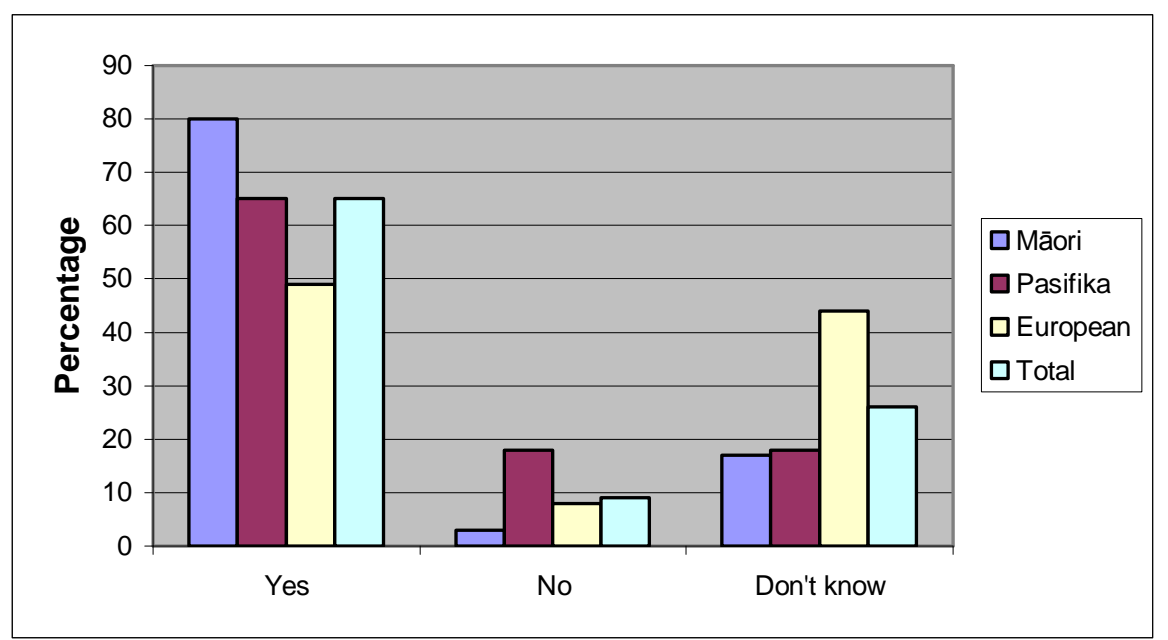

Figure 7.6: Responses to 'My teacher gives me feedback about my maths progress' (Questionnaire 3)

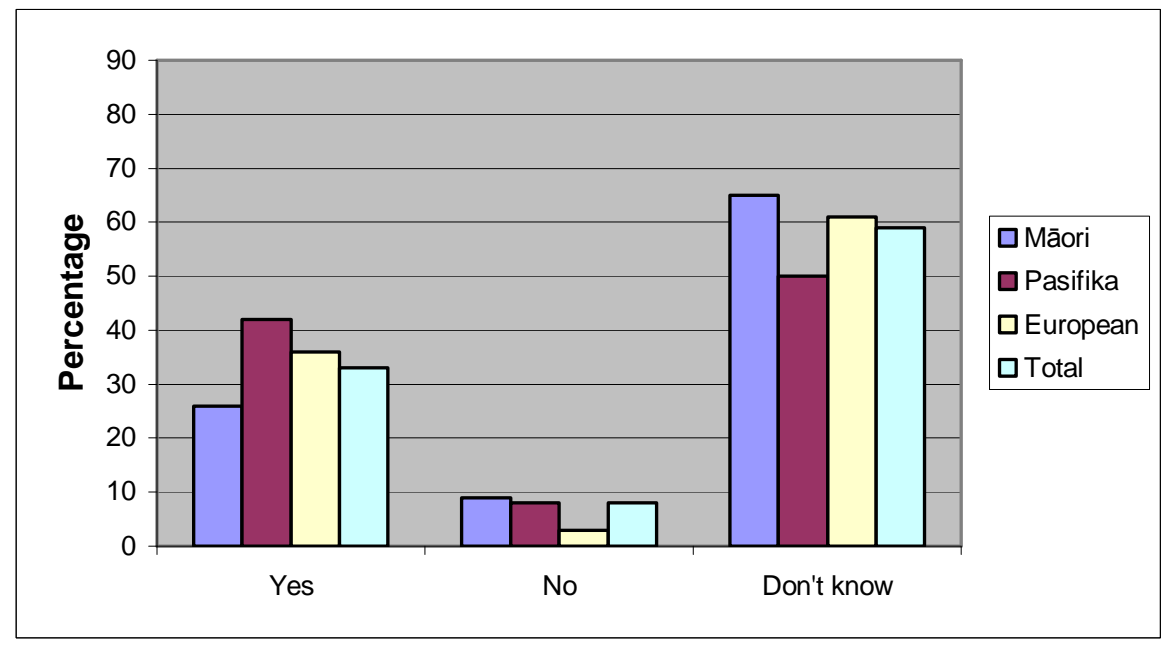

Figure 7.7: Responses to 'My teacher knows how good I am at maths' (Questionnaire 1) 


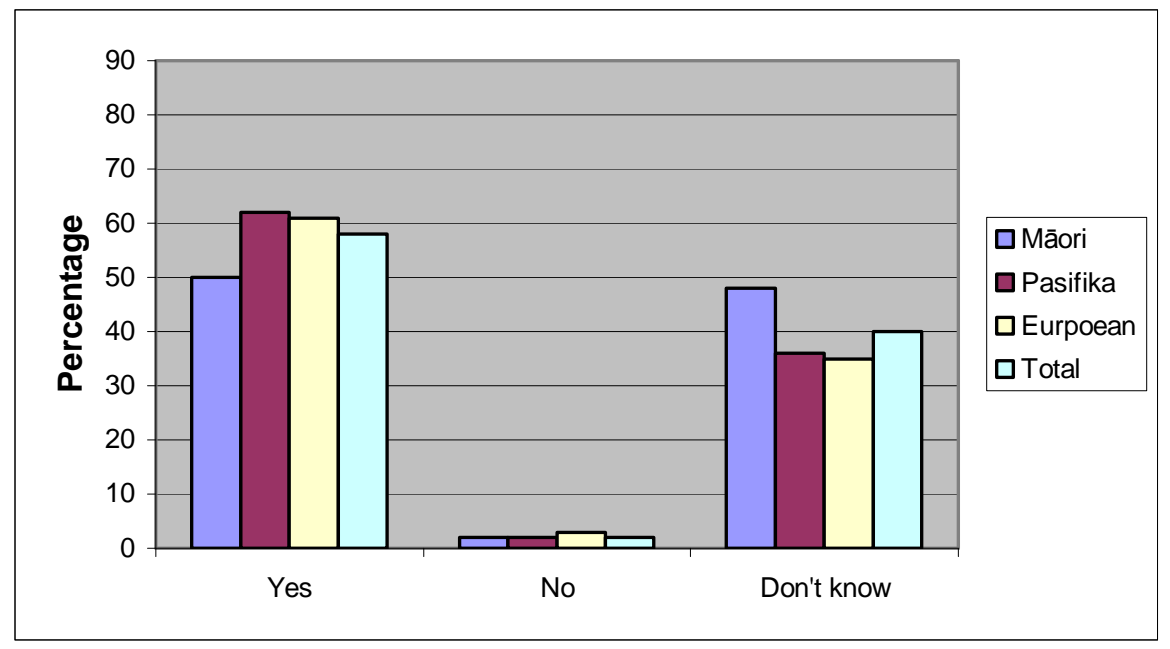

Figure 7.8: Responses to 'My teacher knows how good I am at maths' Note: Mean \% for Questionnaires 2 and 3

SS carried out two formal diagnostic assessment tasks (asTTle ${ }^{36}$ and $\mathrm{PAT}^{37}$ tests) at the beginning of the year. The asTTle results were promptly returned to students. However, the data from her class regarding their teacher's knowledge of their mathematical capability were not markedly different to the classes that had not undergone diagnostic testing. It seems vital that students feel confident that they and their teacher know their mathematical ability. More research is needed to explore how students' impressions of their teacher's knowledge of their mathematical capabilities are created.

Māori students were more likely than the other ethnic groups to report receiving feedback about their learning (Figure 7.6) but were less confident than other groups that their teacher knew their mathematics ability (Figures 7.7 and 7.8). New Zealand European students were least likely to state their teacher gave them feedback about their learning.

The majority of students liked getting written or oral feedback about their progress (over $60 \%$ and over $70 \%$ respectively) but held differing views regarding how they liked to receive it (Figures 7.9 and 7.10).

${ }^{36}$ asTTle is a nationally available electronic literacy and numeracy assessment package.

37 Progressive Achievement Tests (PAT) tests are nationally available assessment tools commonly used as diagnostic assessments by New Zealand schools at the beginning of the school year. 


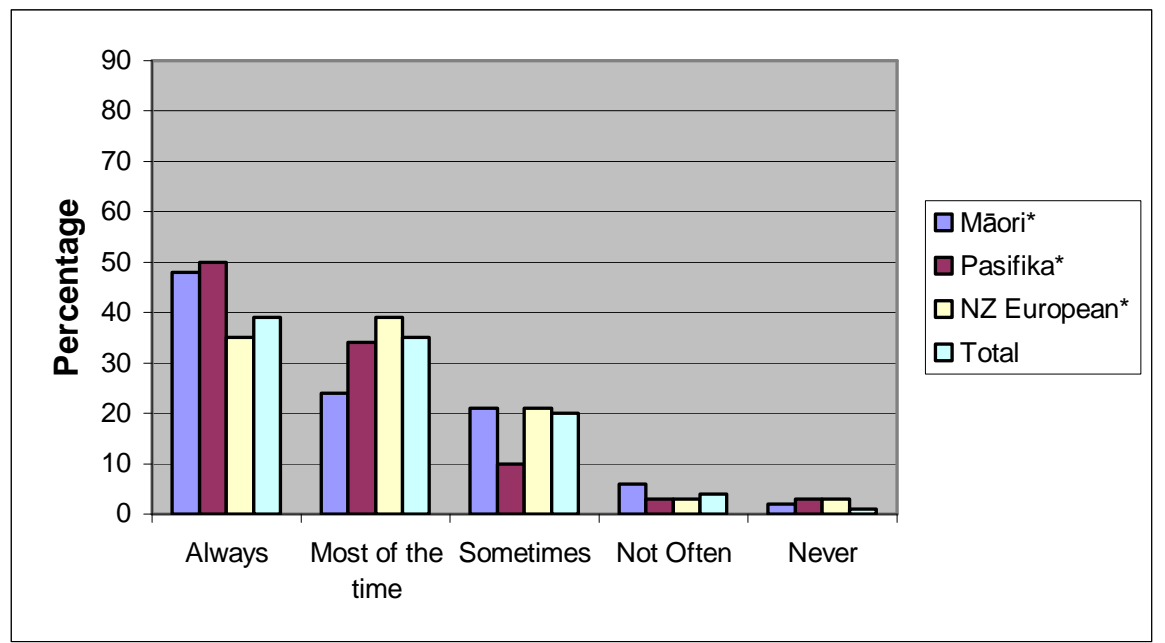

Figure 7.9: Responses to 'I like getting written feedback about my maths progress'

The Pasifika* students were generally more positive than students of other ethnic groups about getting feedback about their learning and there is some evidence that the Māori* and European* students preferred oral feedback to written feedback (Figures 7.9 and 7.10).

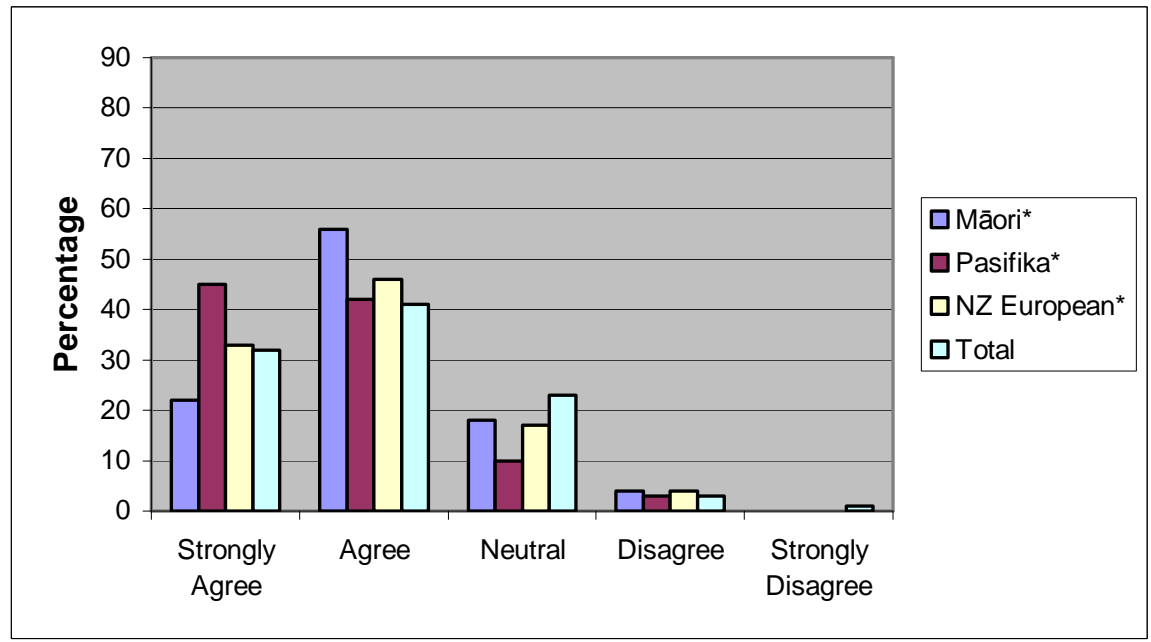

Figure 7.10: Responses to 'I like my teacher telling me about my maths progress'

Closer inspection of the data revealed that $58 \%$ of students overall indicated no preference between receiving feedback about their mathematics learning orally or in written form. One quarter of the students preferred oral feedback and $15 \%$ preferred written feedback. The student preferences and student comments indicate the value of teachers knowing their students' learning needs. Teachers' time could be wasted and the positive effects of their feedback on learning lost if they gave feedback in ways their students did not find accessible:

I like him to tell me all about it because sometimes I don't understand by looking or reading on a piece of paper. (WW student) 
It's easier for me when he explains something when he's standing right next to me, talking just to me. (WW student)

I like it when she says good things about my work and about my potential. (SS student)

I like getting feedback through my work being marked. (SS student)

I like getting letters to my parents about my maths successes. (UU student)

Very few students reported feeling too uncomfortable to ask the teacher about their progress (Figure 7.11); however, almost half expressed the importance of privacy regarding their teacher's comments about their work (Figure 7.12).

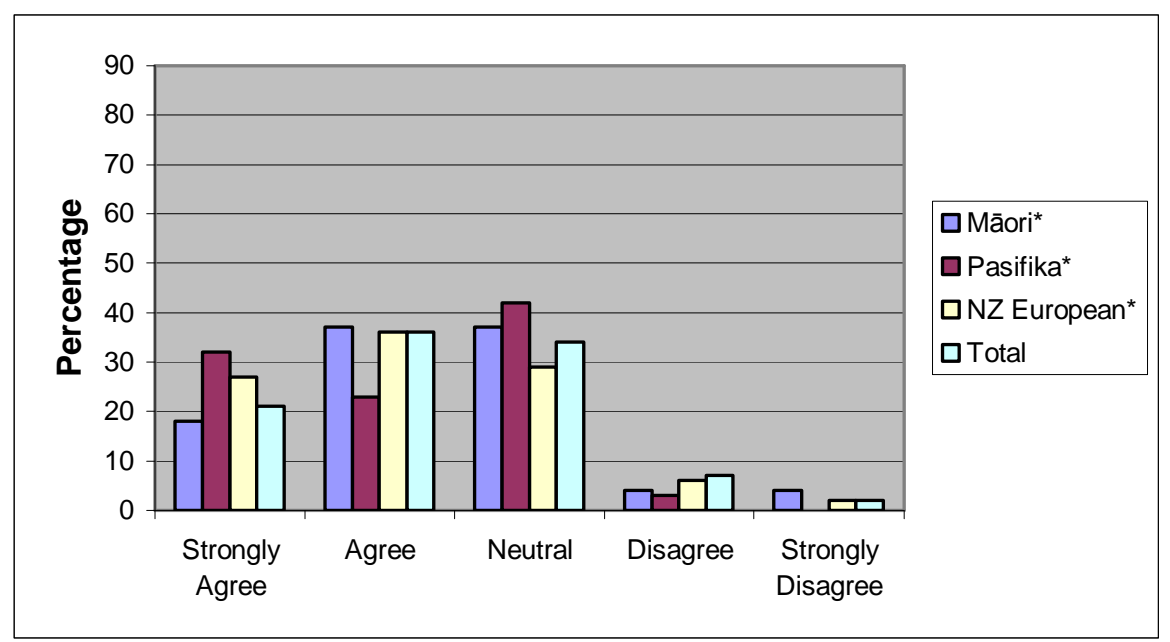

Figure 7.11:

Responses to 'I am happy to ask my teacher about my maths progress'

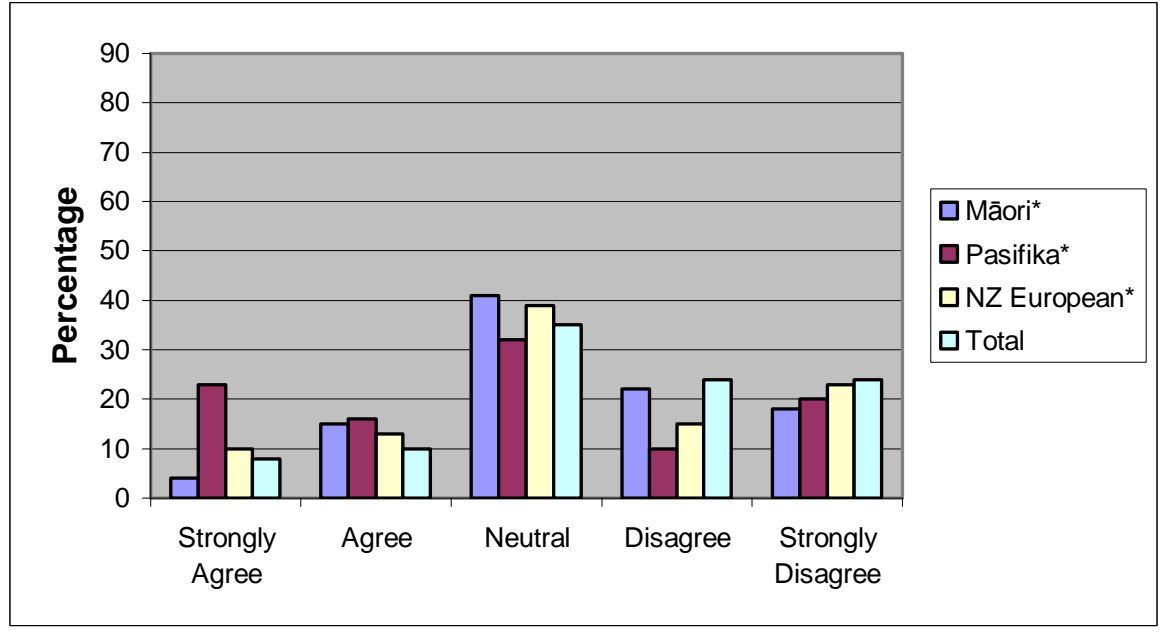

Figure 7.12:

Responses to 'I like my class to hear the teacher's comments about my work'

One student's comment indicated (consistent with the researcher's perception from lesson observations) that they received less privacy than they would have liked during their teacher's oral feedback: 
I like to get the feedback privately, not so the whole class knows. (SS student)

Pasifika* students were slightly more likely, and Māori students slightly less likely than others to be both comfortable to ask the teacher about their progress and to have others hear the teacher's comments about their work (Figures 7.11 and 7.12).

Students were asked in all questionnaires about their motivations for working hard in mathematics. Given a set list of possible motivators, all ethnic groups attributed similar levels of significance to similar motivators. 'Wanting to be successful in NCEA ${ }^{38}$ was the most important motivator for all groups (over $80 \%$ ). Between $60 \%$ and $80 \%$ of students indicated as important: 'my parents expect me to'; 'I want to learn the work'; 'it will be useful for my job in the future'; and 'it will be useful for me in life other than for a job'. 'because my parents expect me to' was selected slightly less often by Māori* students than other groups (63\% across Terms 1-3 for Māori compared with $75 \%$ for others). Roughly $40 \%$ of students indicated 'wanting to work well for their teacher' was a factor that contributed to their working hard, with slightly higher proportions for Pasifika* and slightly lower proportions for Māori* students. 'Because my friends work hard' was selected least often; by roughly $20-30 \%$ of students in each ethnic group.

Teacher characteristics that students stated enhanced their motivation to learn related to teacher behaviour, manner, and sense of purpose. Questionnaire responses indicated that students wanted teachers to interact and co-operate with them and be themselves in class. They wanted teachers who were kind, patient, relaxed, not too serious, and who were able to have fun with the class. Students expressed appreciating being able to share in decision-making. Two quotes in particular exemplified many students' feelings:

Enjoy your job and enjoy our company. (SS student)

Teachers should feel they want to teach us. (UU student)

\subsection{Discussion}

Overall this chapter indicates the importance of teachers knowing and caring about their students. The study students had different sets of priorities for what they wanted their teacher to know, how they wanted their teacher to find it out, how they preferred to learn, how they could be comfortable in class to express themselves,

${ }^{38}$ External assessment in Year 11 
and how they liked to receive feedback about their learning. A few expected that teachers should care for students 'as family', a deeper level of care than many teachers may expect or be prepared to give. Consistent with the literature (Chapter Two), the study results indicate that students' engagement with learning and mathematical success are likely to be enhanced by teachers knowing their students as individuals and as individual learners.

Many students' responses regarding what makes for a caring teacher reflected their general impressions about who the teacher is for them. Many responses were given in terms of their teachers' knowledge of and care for them and their learning, within the themes of liking, respecting, being tolerant of one another, and being able to reflect their personal identities rather than as specific behaviours that their teacher did or did not display. The data indicated that the students' impressions of their teacher's capabilities as a class manager, motivator, person, and teacher (Chapter Five) are inter-related and that mutual teacher-student respect is important for learning. The lesson observations indicated that students show respect for their teacher by listening, willingly complying with requests or suggestions, engaging with learning, and trying to learn well.

Some differences in opinions were found across ethnicity. There could be many reasons why differences by ethnicity occur including inherent cultural differences in attitudes and values. One possible interpretation of the greater feelings of teacher respect experienced by Māori and Pasifika students could be that these students may be more sensitised to whether or not they are respected. 


\section{CHAPTER EIGHT}

\section{Students as Culturally Located Individuals}

This chapter describes students' perceptions of: their cultural identities (Section 8.1); the importance their schools and teachers place on knowing and understanding their cultural identities (Section 8.2); and students' perceptions of links between students' cultures and mathematics learning (Section 8.3). Finally, the discussion draws overall conclusions from the results presented in this chapter and returns to the remaining themes of the whāriki (Figures 3.5 and 6.1): knowing one another's cultures; and enhancing feelings of cultural identity (Section 8.4).

Throughout the study students showed confusion between ethnicity, culture, and ability with a heritage language. Furthermore, students' overwhelming lack of experience (or lack of recognition) of mathematics learning experiences drawn from Māori or Pasifika contexts in their current and previous mathematics learning was clearly apparent. Therefore many students' responses in this area are based on their hypothesising what this may be like rather than on knowledge and awareness gained from personal experiences.

The number of students who stated similar ideas is shown in several sections of this chapter in order to indicate the relative prevalence of responses. For example, a comment followed by (M 3P 2E) indicates that one Māori student, three Pasifika, and two New Zealand European students made the comment (or an equivalent statement).

\subsection{Students' cultural identities}

This section presents results regarding students' depth of engagement with their heritage cultures (Section 8.1.1) and how relevant students believe their culture is to their mathematics learning (Section 8.1.2).

\subsubsection{Students' depth of engagement with their heritage culture/s}

Students were asked to list both the languages they spoke and the languages they used at home as rough indicators of the depth of engagement with their heritage culture/s, consistent with Blum's measure of engagement with heritage culture (1999). Fifty-five percent of those who identified as Māori๋ indicated they spoke some level of te reo Māori and 15\% spoke Māori at home. Corresponding figures for 
those identifying Pasifika* heritage were $76 \%$ and $49 \%$. Lesson observations and discussions with study teachers indicated that most students were fluent English speakers. Using this indicator, the New Zealand European students were those most deeply engaged with their cultural heritage, followed by the Pasifika students and lastly, the Māori students.

However, when asked to identify things special to their culture $73 \%$ of responses (99 of the 136 who completed Questionnaire Three) gave no reply or did not know (85\%, $60 \%, 76 \%$ and $71 \%$ of the responses respectively for Māori, Pasifika, New Zealand European, and Other students). While the low response rate was similar for other questions requiring a written response (rather than a tick box or verbal response), of the $27 \%$ (37 students) who did reply, two appeared ambivalent (don't know, don't care (M E)) and seven showed some confusion about the question, i.e., 'List things special to your culture':

I'm Māori. (M)

Cultural stuff, things that have to do with my culture. (M)

I'm white so I guess I don't have anything special about my culture. (E)

Well, I'm white, so I dunno (E)

I'm not religious at all. (E)

I'm half NZ, quarter Italian and quarter Polish and I love it! (E)

Everything? (P)

Um, how does this relate to - oh, never mind. $(\mathrm{N})$

The remaining 28 students ( $21 \%$ of those completing the questionnaire) replied with specific ideas about things special to their culture in the areas of:

- people;

Family (M 3P 4E), Friends (P 4E), Ancestors (E), The people in our iwi (tribe) (M), Kaumatua (tribal leader) (M);

- possessions;

What my family owns in the Pacific Islands, including land $(P)$, Land $(M)$, Clothing $(P)$;

- knowledge;

Language (4P), Knowing our traditions (M 2P), Knowing our protocols (P), Knowing everyone (O), That I know where I come from (M), To remember your relatives are suffering $(\mathrm{O}), \mathrm{I}$ can believe what I want and choose to go to church $(M)$, Knowing I am learning for me and no-one else $(E)$; 
- activities;

Food (4P), Performances (M), Samoan dancing (P), Performing arts and cultural dancing like kapahaka (M), Family holidays, celebrations, outings, feasts, $(E)$, Being successful in life (P O), Big achievements $(E)$, School $(P)$, I'm white, I do white people things (E);

- traditions;

Tattoos (P), Koru (stylised fern frond) (M), Moko (facial tattoo) (M), Communicating (O), If an old woman or an old man dies you have to take money to the family of the person who died and they have to wear lavalava (wrap around material used as a skirt) $(\mathrm{P})$;

- religion;

Religion (O), Christianity $(\mathrm{N})$, Diwali and Eid and festivals $(\mathrm{O})$, I believe in God and try to walk with him most of the time $(\mathrm{E})$;

- attitudes;

Respect (4P E), Being treated equally $(P)$, Attitude $(P)$, Respecting elders $(E)$, Pride $(P)$;

and

- behaviours.

Treating others the way you would like to be treated in return (E), Being well disciplined at all times so if we do good or bad things it reflects on our parents... this goes for any culture (N), I am Māori and I never talk in class and that's my culture $(M)$, It is rude to point $(N)$, Doing as I'm told (E), Being lazy is my culture and the same for lots of others $(E)$.

The interview and questionnaire responses indicated that the New Zealand European students were less able to identify aspects of their heritage culture, more often confused by questions regarding culture, and more likely to see themselves as comparatively culture-free. This is likely to be a result of being immersed in their culture (the dominant New Zealand culture) on a daily basis:

Do you think it's important for your maths learning that your teacher knows about your culture? (Interviewer)

Well I don't really have a culture where we do many things, you know. I guess for students that may be Māori and stuff like that and they have their traditions and stuff but not really for me. (from interview with two UM students, E O males)

I don't have a culture, do I? (UM student, E female) 
Despite the variation in the depth of engagement with their cultural heritage, the vast majority of students, regardless of their ethnic group, felt that their culture was very important to them (Figures 8.1-8.3).

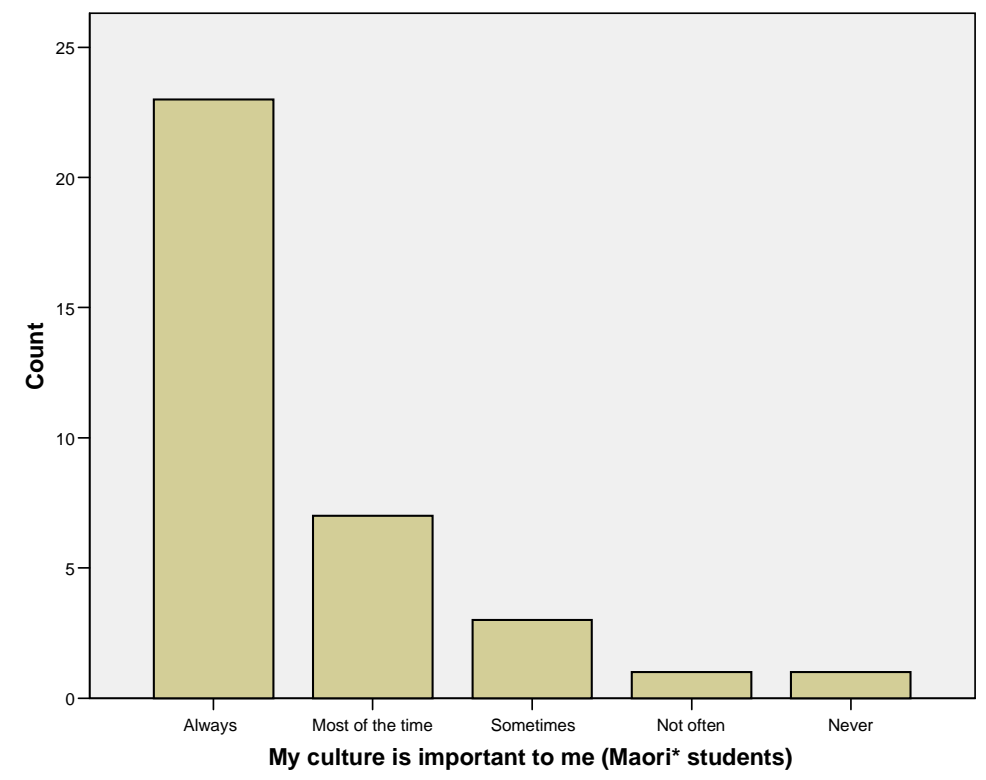

Figure 8.1: My culture is important to me (Māori ${ }^{\star}$ students)

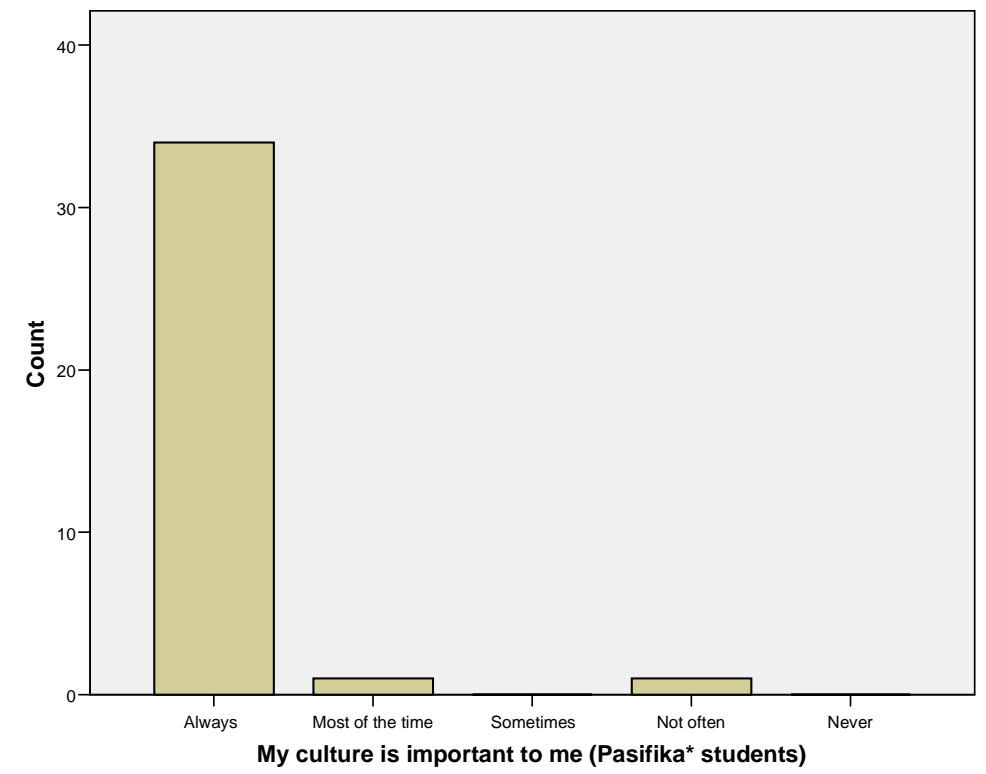

Figure 8.2: My culture is important to me (Pasifika* students) 


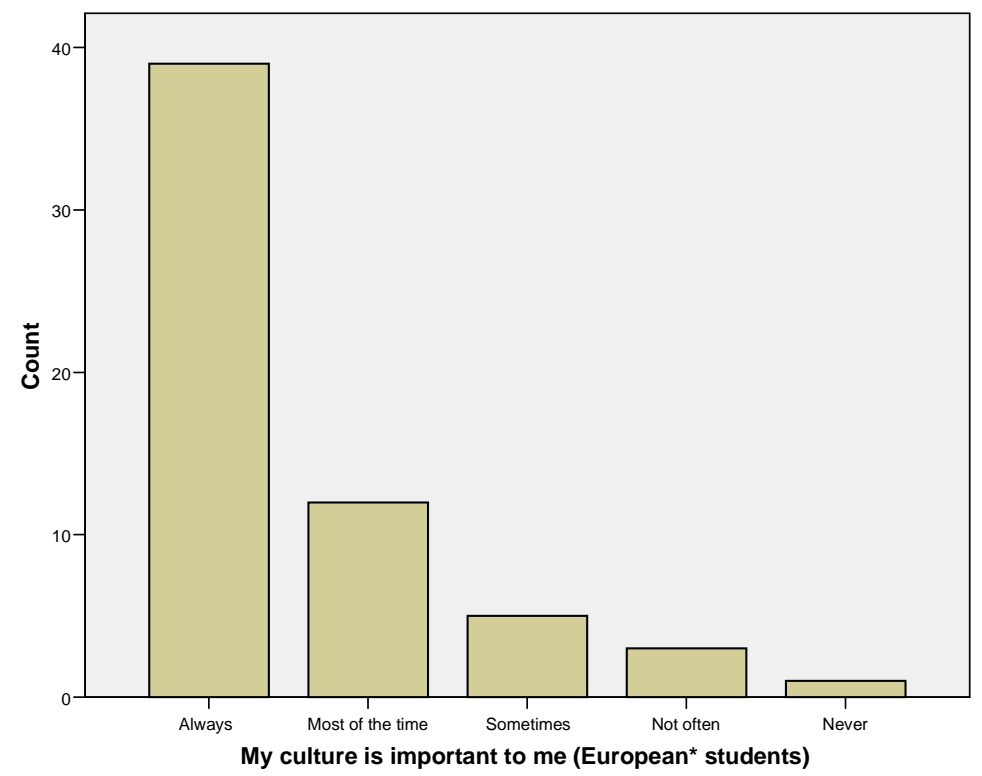

Figure 8.3: My culture is important to me (New Zealand European* students)

\subsubsection{Students' perceptions of the relevance of their heritage culture to their mathematics learning}

At the Pasifika cultural advisor's suggestion, students' views of the relevance of their culture to themselves, their learning in general, and their mathematics learning were probed through the addition of a range of questions in Questionnaires Two and Three. The rationale for including extra questions was to understand more clearly what appeared to be confusion and lack of consistency in the student data previously gathered in this area of the study. Cultural advisors were consulted regarding the increased focus and additional questions. Results from each set of questions are presented in turn in this section and are followed by overall conclusions about the results.

A measure of the relative importance students placed on their heritage culture in comparison to other groups they belonged to was obtained using Questionnaire Three (Appendix 6). Parallel questions were included in the corresponding teacher questionnaire (Section 6.3). Students were asked the importance of different groups for their life and for their mathematics learning, indicating the importance of each group to them using a four-point scale (0-3 points). Overall scores for each group were calculated by adding together the total number of points. Similar to their teachers, students ranked their heritage culture in the top five groups for importance in both their lives and their mathematics learning (Table 8.1, Figure 8.4). Heritage culture was seen as more important to students' lives $\left(3^{\text {rd }}\right)$ than their mathematics learning $\left(5^{\text {th }}\right)$. 
Table 8.1: Importance to students of the groups they belong to

Note: Students indicated the relative importance to them of each group using 0,1 , 2, or 3 check marks. Overall scores for each group were calculated by adding together the total number of check marks for each group.

\begin{tabular}{|c|c|c|}
\hline $\begin{array}{l}\text { Rank } \\
\text { order }\end{array}$ & $\begin{array}{l}\text { Importance to my life: } \\
\text { (total \# points) }\end{array}$ & $\begin{array}{l}\text { Importance to my maths learning: } \\
\text { (total \# points) }\end{array}$ \\
\hline $1^{\text {st }}$ & My family (297 points) & My class group (185 points) \\
\hline $2^{\text {nd }}$ & My school friends (267) & My school friends (172) \\
\hline $3^{\text {rd }}$ & My heritage culture, my ethnicity (203) & My year level group (151) \\
\hline $4^{\text {th }}$ & My class group (192) & My family (140) \\
\hline $5^{\text {th }}$ & My year level group (176) & My heritage culture, my ethnicity (71) \\
\hline $6^{\text {th }}$ & My sports teams (167) & My church group (43) \\
\hline $7^{\text {th }}$ & My church group (132) & My sports teams (38) \\
\hline $8^{\text {th }}$ & $\begin{array}{l}\text { Local community youth (out of school) } \\
\text { (118) }\end{array}$ & $\begin{array}{l}\text { Local community youth (out of school) } \\
\text { (27) }\end{array}$ \\
\hline $9^{\text {th }}$ & My clubs and other interests (111) & My clubs and other interests (25) \\
\hline $10^{\text {th }}$ & NZ youth e.g., through TV and radio (75) & NZ youth e.g., through TV and radio (17) \\
\hline
\end{tabular}

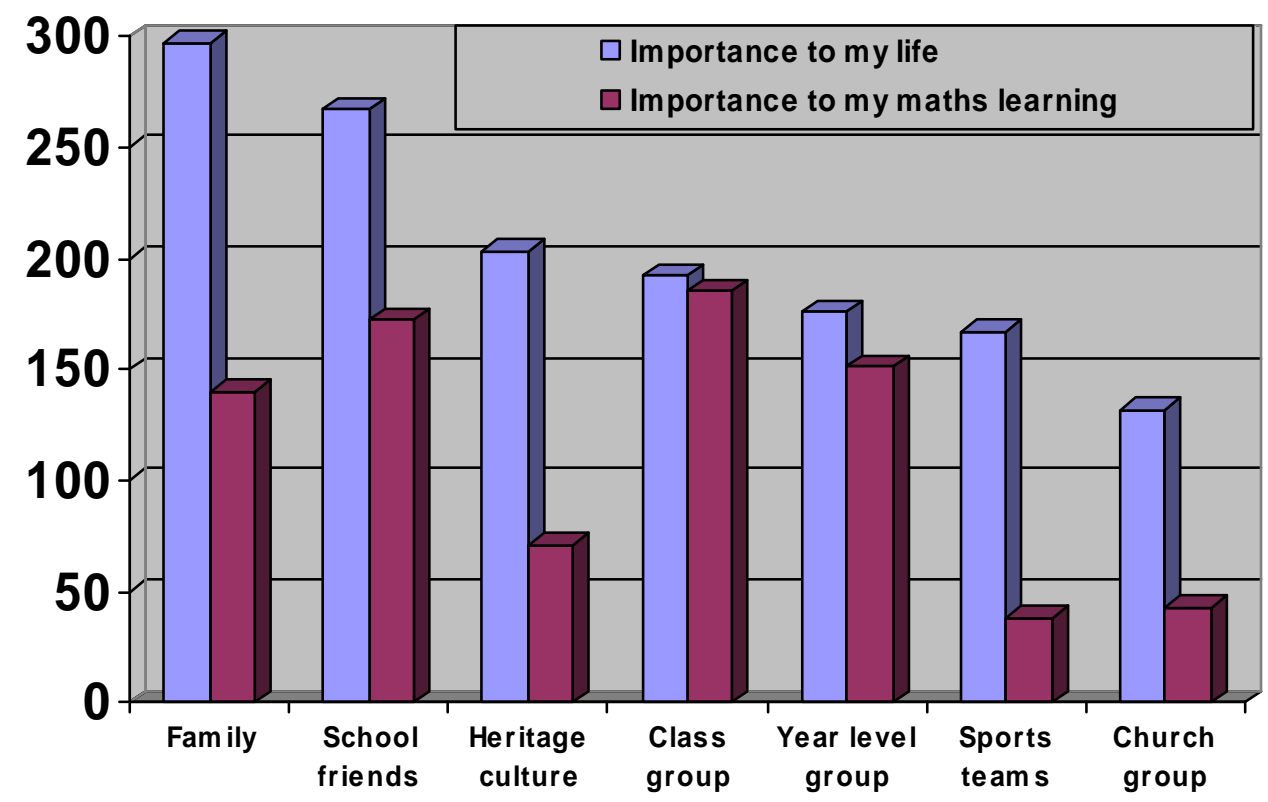

Figure 8.4: Importance to students of the groups they belong to Note: The figure includes the seven groups with the highest frequencies.

Another way to explore students' feelings regarding the relevance of their cultures at school and to their mathematics learning used descriptors with a 5-level Likert scale to indicate the strength of students' beliefs (Figures 8.5 and 8.6 respectively). 


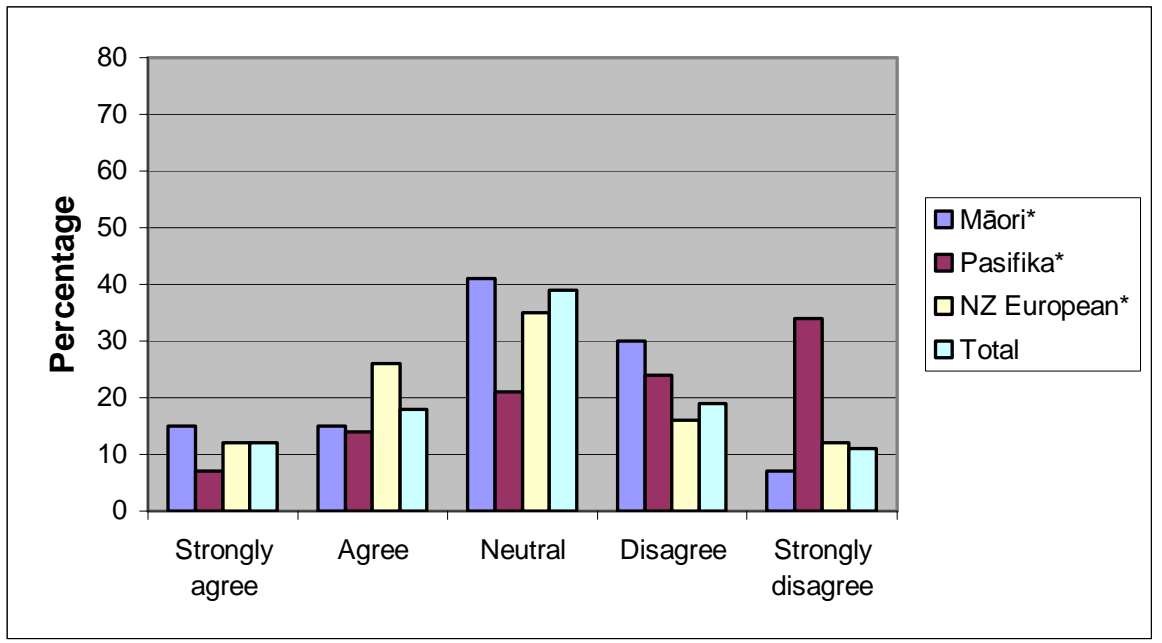

Figure 8.5: Responses to 'My culture is not relevant at school' (\%)

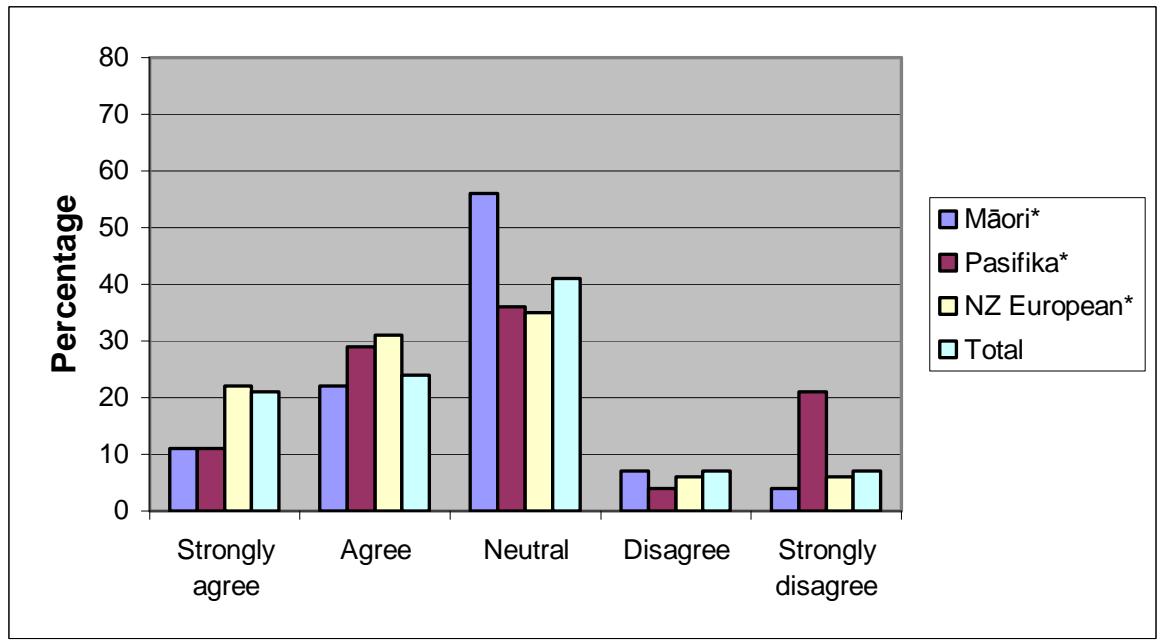

Figure 8.6: Responses to 'My culture is not relevant to my maths learning' (\%)

The results to these questions showed that overall roughly $40 \%$ of students were neutral regarding both descriptors. The remaining $60 \%$ were fairly evenly spread regarding whether or not their culture was relevant at school; however, they felt their culture was less relevant to their mathematics learning than it was at school generally (Figure 8.6). The main difference across ethnic groups was that:

- Pasifika* students were more likely than Māori* and European* to believe their culture was both relevant at school (59\% compared with $37 \%$ and $28 \%$ respectively) and to their mathematics learning (25\% compared with $11 \%$ and $12 \%$ respectively).

When students were asked whether or not it was important their teacher know about their culture, one responded:

I think it's good to know for them to know what your cultural identity is and what one you belong to but for them to fully understand it, that's not really necessary 'cause as long as they know who you are, they don't need to know everything about you. (WK student) 
The majority of students (54\%) were unsure about whether or not it is important for their mathematics learning that their teacher cares about their culture (Figure 8.7); however, one fifth overall replied that it is important, with higher proportions of Māori* and Pasifika* than New Zealand European* students in this group (Figure 8.7).

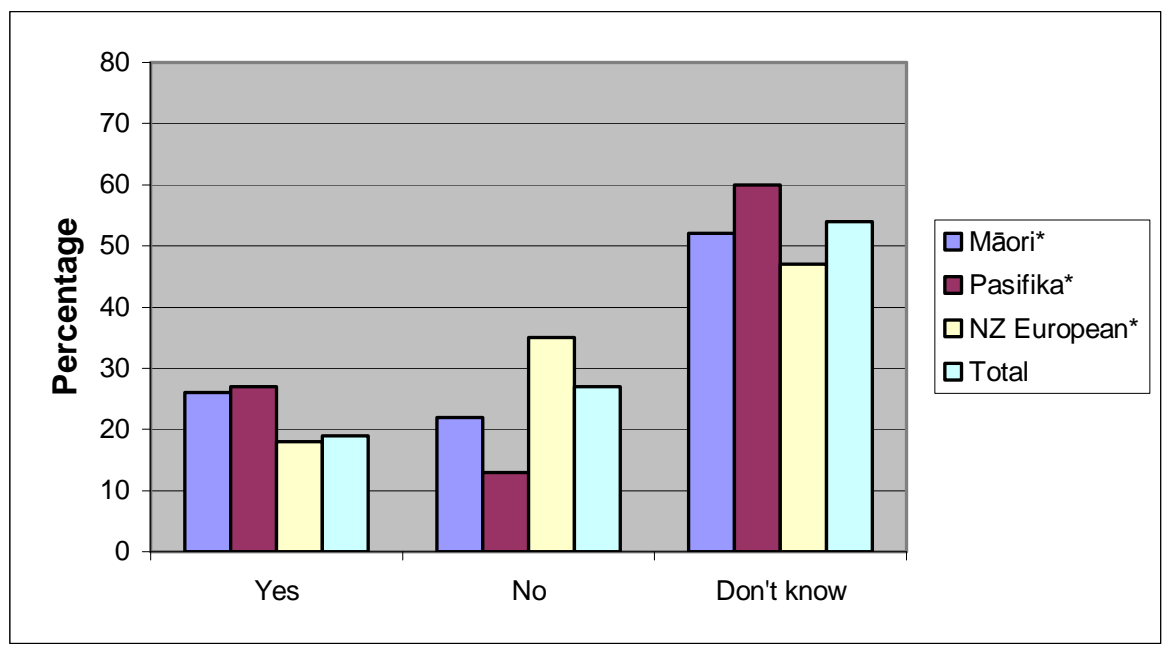

Figure 8.7: Responses to 'It is important for my maths learning that my teacher cares about my culture' (\%)

Once again, Pasifika* students were more likely and European* students less likely to believe in the relevance of their culture to their mathematics learning. Reasons given to support their feelings (paraphrased) included:

- Yes; so that the teacher would be inclusive (M 3P 4E), because culture is an integral part of who we are (M 2P E), so they could reflect class cultures in learning examples $(\mathrm{M})$, because we might learn in different ways $(\mathrm{N})$, and to enhance students' personal feelings of cultural identity $(\mathrm{N})$;

- No; because teachers should treat everyone the same (P 3E), culture and maths are two separate things (2M P 7E N), our culture should not matter when we are learning maths (M P 3E), it is none of the teacher's business $(P)$, teachers are already too busy without having to learn about students' individual cultures (M); and

- Do not know; because culture and maths are two separate things (2P $3 \mathrm{~N})$, our cultures do not affect our learning (2M 2P E), and teachers should not teach differently because people have different cultures (E).

Again students' responses indicate that many see their learning (in particular their mathematics learning) and their culture as separate and hint that being responsive to culture in mathematics learning may positively or negatively alter equity of access to learning opportunities. Again some confusion between culture, language, and ethnicity was apparent:

No, not really, as long as the student knows basic English she/he will be sweet. (E) 


\subsection{Students' perceptions of the importance placed on heritage cultures at school}

This section describes students' perceptions of the value placed by their school and teacher on students' cultures (Section 8.2.1) and their teachers' knowledge of their cultures and the Māori and Pacific worlds (Section 8.2.2).

\subsubsection{Students' perceptions of the importance placed on students' culture by their school and by their teacher}

Students were asked to indicate the importance placed on students' cultures by their school and their teacher (Appendix 6). Forty percent of students responded that they did not know whether or not students' ethnicities/cultures were important to their school and $61 \%$ that they did not know if students' ethnicities/cultures were important to their teacher. Of those who expressed an opinion, there was no consistency across or within schools by gender or ethnicity of students' perceptions of the extent to which students' cultures are important at their school. In general, most felt their school placed medium to high importance on their culture and their teacher considered their ethnicities/cultures as fairly important. Compared to Samasama and Whero, Uliuli's results regarding the importance their teachers placed on their ethnicities/cultures were fairly neutral.

Many students indicated one or other of both extremes (very important, not important at all), and most stages between the extremes were indicated by others. Reasons given for stating their school placed medium to high importance on their culture (Table 8.2 ) related to:

- opportunities to join and/or watch cultural groups and performances;

- the presence of many cultural groups in the school;

You can just tell by all the out front cultures we do have, teachers and students. $(P)$

We are a very multicultural school that respects each and every person's ethnicity. (P E)

We have a lot of different cultures at our school and the school caters for them. (N)

- the respect shown for students and their cultures; and

- the use of Māori or Pacific languages (e.g., by school leaders at assemblies) or by school policy decisions (e.g., opportunity to study languages, assistance by translators when needed). 
Table 8.2: How study schools show that cultures are important

Note: $\quad$ Examples were given by 71 of the 136 (52\%) students who completed the questionnaire some of whom listed more than one item.

\begin{tabular}{|l|c|c|c|}
\hline \multicolumn{1}{|c|}{ Aspect } & Samasama & Whero & Uliuli \\
\hline Cultural activities & 14 & 11 & 4 \\
\hline $\begin{array}{l}\text { Language use and } \\
\text { classes }\end{array}$ & 3 & 3 & 2 \\
\hline $\begin{array}{l}\text { Respect/lack of } \\
\text { racism }\end{array}$ & $\begin{array}{l}\text { 2 } \\
\text { ('Social Studies is } \\
\text { sometimes on } \\
\text { different cultures', 'by } \\
\text { allowing students } \\
\text { over 17 to study at } \\
\text { the school') }\end{array}$ & $\begin{array}{l}\text { ('people staying close } \\
\text { together') }\end{array}$ & $\begin{array}{l}\text { ('we have a lot of } \\
\text { different cultures at } \\
\text { our school and the } \\
\text { school caters for } \\
\text { them') }\end{array}$ \\
\hline $\begin{array}{l}\text { Other } \\
\text { (t) }\end{array}$ & - & - & $\begin{array}{l}1 \\
\text { Marae }\end{array}$ \\
\hline $\begin{array}{l}\text { Sotal number of } \\
\text { responses }\end{array}$ & 23 & 19 & 1 \\
\hline
\end{tabular}

Reasons for believing their school did not place importance on students' cultures included:

They sometimes talk about my culture in a bad way. $(\mathrm{N})$

The only thing that this school does to represent my culture is Polyclub. ${ }^{39} \mathrm{We}$ need Cook Island classes. (P)

The Māori teacher shows that she cares, but not the other teachers. (M)

There were 17 questionnaire responses regarding ways in which their mathematics teachers showed students' cultures are important, less than one quarter of the number describing how their schools did so (72 responses). The lower response rate is perhaps not surprising given the nature of many of the school-based responses (e.g., cultural activities and language classes). However, of concern is that there was a higher rate of negatively framed responses regarding teachers than schools ( $24 \%$ for teachers compared with $6 \%$ for schools).

Ways the students reported their mathematics teachers showed the importance of students' cultures (Table 8.3) included themes of respect (see also Section 9.1.3), treating everyone in the same way, treating students of different cultures in different ways, giving help when needed, and trying to learn words in the students' heritage languages.

${ }^{39}$ Performing arts group 
Table 8.3: How study teachers show that cultures are important

\begin{tabular}{|c|c|c|c|}
\hline Aspect & Samasama & Whero & Uliuli \\
\hline $\begin{array}{l}\text { Respects cultures, } \\
\text { treats everyone the } \\
\text { same }\end{array}$ & 1 & 3 & 2 \\
\hline $\begin{array}{l}\text { Treats students } \\
\text { differently according } \\
\text { to culture (positively) }\end{array}$ & \begin{tabular}{l}
\multicolumn{1}{c}{1} \\
('helping the kids \\
who have problems \\
speaking English')
\end{tabular} & \begin{tabular}{l}
\multicolumn{1}{c}{3} \\
('he takes time to \\
learn most languages \\
and even though he \\
only knows a little \\
Samoan he enjoys it')
\end{tabular} & - \\
\hline $\begin{array}{l}\text { Treats students } \\
\text { differently according } \\
\text { to culture } \\
\text { (negatively) }\end{array}$ & \begin{tabular}{l}
\multicolumn{1}{c}{1} \\
('the teacher sends \\
out all the Islanders \\
when they talk but \\
when the Pākehāa \\
talk nothing happens \\
to them)
\end{tabular} & - & - \\
\hline $\begin{array}{l}\text { Doesn't do anything, } \\
\text { I don't know }\end{array}$ & 3 & - & 1 \\
\hline $\begin{array}{l}\text { Doesn't care about } \\
\text { students' cultures }\end{array}$ & - & - & 2 \\
\hline $\begin{array}{l}\text { Total number of } \\
\text { responses }\end{array}$ & 6 & 6 & 5 \\
\hline
\end{tabular}

The apparent inconsistency between student statements regarding positive teacher behaviours (i.e., treating everyone the same and treating them differently according to their culture) was explored further in interviews with students (Section 8.2.2) but is worthy of further examination. It may be that many students believe both treating students the same and differently to be equitable, depending on context. Comparison of teachers' and students' views regarding treating students in the same or in different ways within specific contexts would help illuminate this result. Teacher responsiveness to students' interactions and body language, and their knowledge of cultures and cultural difference are likely to assist them in making classroom decisions deemed by their students as suitable and caring. One student's insightful suggestion has the potential to help develop teachers' cultural knowledge, the importance they place on students' cultures, and how they show it:

Teachers could have a good ethnic buddy. (P)

The results in this section highlight the wide range of student views regarding the relevance of their culture at school and by their teacher. Low response levels may be related to the apparent variation in students' cultural awareness and may indicate many students had not previously deeply considered the place of deliberate acknowledgement of students' cultures in school settings.

${ }^{40}$ Student's term for people with Pasifika heritage 


\subsubsection{Students' perceptions of their teachers' knowledge of their cultures and the Māori and Pacific worlds}

Corresponding questions from the three questionnaires were designed to indicate differences in teachers' cultural knowledge between students' desires, teacher practice, and over time. Students were asked in Term One to indicate how strongly they agreed with the statement 'I would like my teacher to know my culture' (Figure 8.8). In Questionnaires Two and Three they were asked to indicate whether or not they felt their teacher knew their culture (Figure 8.9).

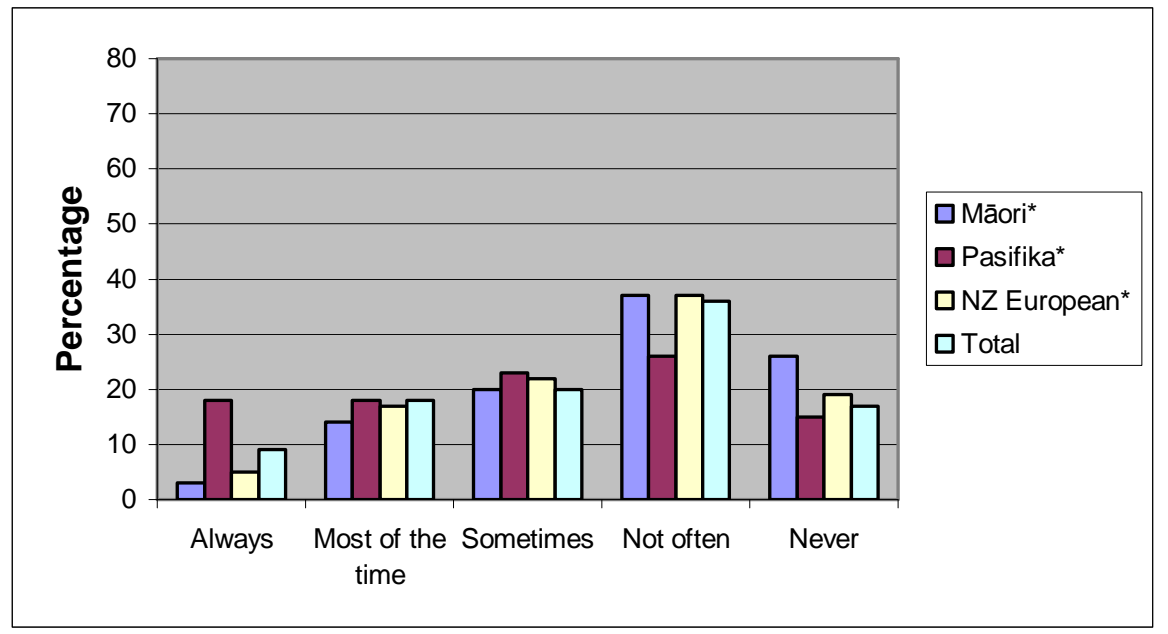

Figure 8.8: Responses to 'I would like my teacher to know my culture' (\%)

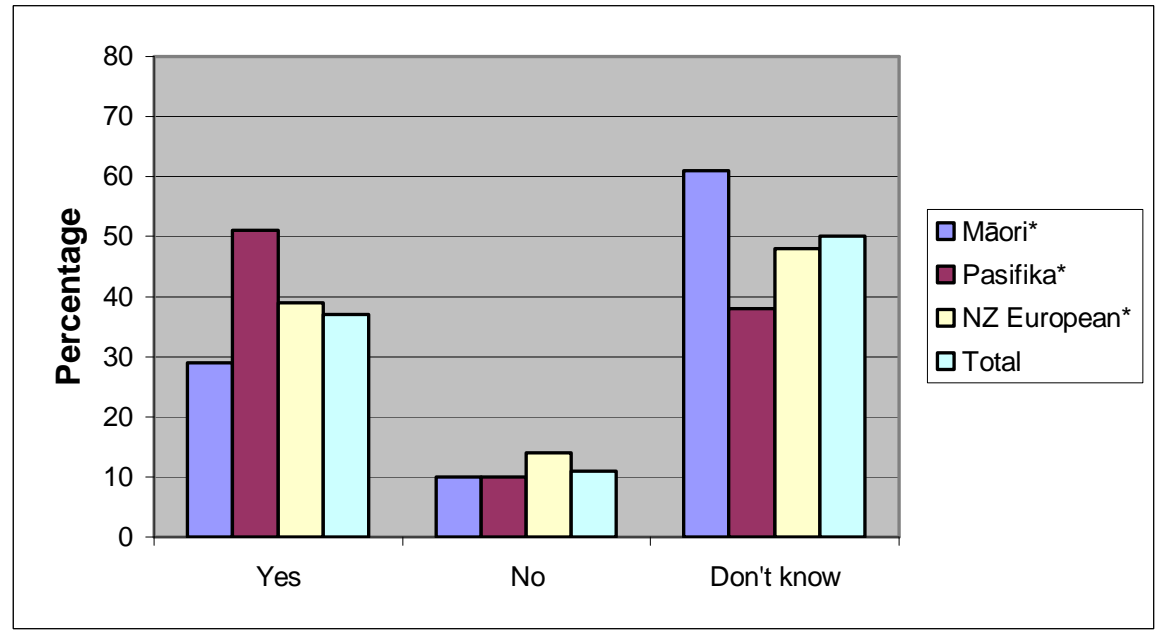

Figure 8.9: Responses to 'My teacher knows my culture' Note: Mean \% for Questionnaires Two and Three

In spite of most students feeling their culture is very important to them, the majority did not want their teacher to know their culture, shown by the high proportions of students selecting 'not often' and 'never' (Figure 8.8). Reasons for students' responses may be linked to whether or not students viewed their culture as relevant to their mathematics learning (Figures 8.6 and 8.7), how they believe their teacher 
would treat such knowledge, and the importance they feel their school places on their culture (Section 8.2.1).

Pasifika* students were more keen than their Māori* and European* peers that their teacher know about their culture (35\% responding 'always' or 'most of the time' (compared with $17 \%$ and $27 \%$ for Māori* and European* respectively) (Figure 8.8 ). Later in the year Pasifika* students were also more likely than other groups to feel their teacher knew their culture (51\% compared with $28 \%$ and $38 \%$ for Māori* and European* respectively). Many students (more than $35 \%$ for all groups), and in particular Māori* students $(61 \%)$, did not know if their mathematics teacher knew about their culture (Figure 8.9).

In contrast to the questionnaire data (Figure 8.8), the students' interview responses were largely positive about the importance of their teacher knowing their culture. The variation in results could in part be as a result of a difference in how the word 'you' was interpreted in the two modes of data gathering that became apparent during data gathering. In the interviews, 'you' was often interpreted in its plural sense in contrast to the questionnaires where it is assumed that students generally interpreted 'you' in the singular. Students' perceptions of the importance to their class of their teacher knowing the class's cultures may have been different to their feelings of the importance of this for themselves as individuals because of their care for one another and their impressions of one another's feelings.

The importance students placed on feelings of respect and not giving offence was reflected in many interview responses:

Do you think it is important that your teacher cares about the cultures in the class? (Interviewer)

Oh yeah. (UU students, E male and $E$ female)

And why is that? (Interviewer)

Because if she's not respectful or whatever, then, I don't know, some people might think it's not fair or something. (UU students, E male and E female)

Thank you Brenda. What do you think James? (Interviewer)

I think if she takes over, like if she makes her class all her own culture then they won't really like learning, so she needs to respect them. (UU students, E male and $\mathrm{E}$ female)

Respect their differences, but to treat everybody the same or treat everybody differently? (Interviewer)

The same but respect everyone's culture. (UU students, E male and E female) 
Yeah the teacher might say something that would offend someone from a different culture, so if she knew that was their culture she wouldn't say it. (UU students, $\mathrm{M}$ female and $\mathrm{E}$ female)

Do you think it is important for your teacher to show they care about people's cultural identities? (Interviewer)

Yes, because that just makes people feel good because they know they are respected. (WK student, $\mathrm{M}$ male)

So he doesn't say the wrong thing. (UM students, E male and O male)

Oh yeah, well if they insulted it, it would make me angry. (ST students, E male and $\mathrm{N}$ male)

Yea, so they don't have to be racist or anything and it doesn't have to affect you in any way about what culture you are. (WK student, $\mathrm{N}$ male)

Student views regarding the importance of their mathematics teachers knowing and caring about students' cultures varied greatly. Some students considered that teachers caring for students' cultures could hinder their mathematics learning:

Is it important for your maths learning that your teacher cares about your cultures? (Interviewer)

I'll say probably no, because if they care about your culture, it will interfere with your maths learning. (WK student, $\mathrm{N}$ )

while others believed that the teacher knowing about their cultures could help students learn:

Is it important for your maths learning that your teacher knows about your cultures? (Interviewer)

Sort of, yes, "cause sometimes people may not understand what he is saying, they might not speak English. (ST students, $2 \mathrm{P}$ female)

Other students believed their teachers did not need to know their cultural identities, some because they viewed mathematics learning and cultural identity as two separate things, and others because they themselves lacked confidence in their own knowledge of their culture or heritage language:

Do you think it's important that your maths teacher knows your culture and your cultural identity? (Interviewer)

Not really, no. (WW students, N) 
No, that doesn't affect our maths. (WW students, N)

Yeah, [culture and maths] are separate things and culture doesn't really go into maths problems or anything. It won't help us if we sit there and talk about our ethnicity or anything. (WK student, $\mathrm{N}$ )

Is it important that your teacher knows what culture you belong to? or doesn't it make a difference? (Interviewer)

Doesn't matter. (ST students, M male and P male)

So for both of you it doesn't matter? Do you see your culture as something separate to the maths classroom or something that is important in the maths classroom? (Interviewer)

Separate. (ST students, M male and P male)

Okay, so can you explain a bit about that... or it just feels like that? (Interviewer)

Feels like that. (ST students, M male and P male)

Do you want it to be separate or would you like to have your own culture in the classroom? (Interviewer)

I kind of want it to be separate. (ST students, M male and P male)

Can you explain why? (Interviewer)

Well I really don't know my own culture, how to speak it. (ST students, M male and $\mathrm{P}$ male)

Students' beliefs about their teacher's knowledge of the Māori and Pacific worlds were also explored in two further sets of Questionnaire questions (Figures 8.10 and 8.11). ${ }^{41}$ In Questionnaire One students were asked whether or not they would like their teacher to know about the Māori and Pacific worlds. In Questionnaires Two and Three they were asked whether their teacher knew about these worlds.

41 The corresponding question 'I would like my teacher to know about the European world' was not asked but in hindsight may have provided useful comparison data. 


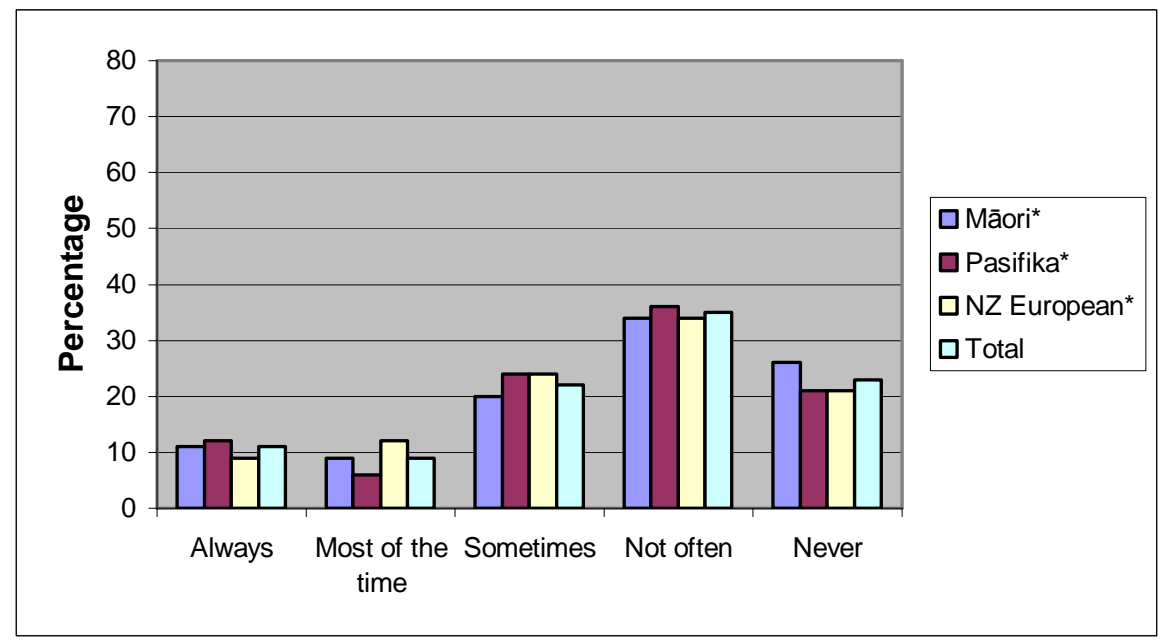

Figure 8.10: Responses to 'I would like my teacher to know about the Māori world’ (\%)

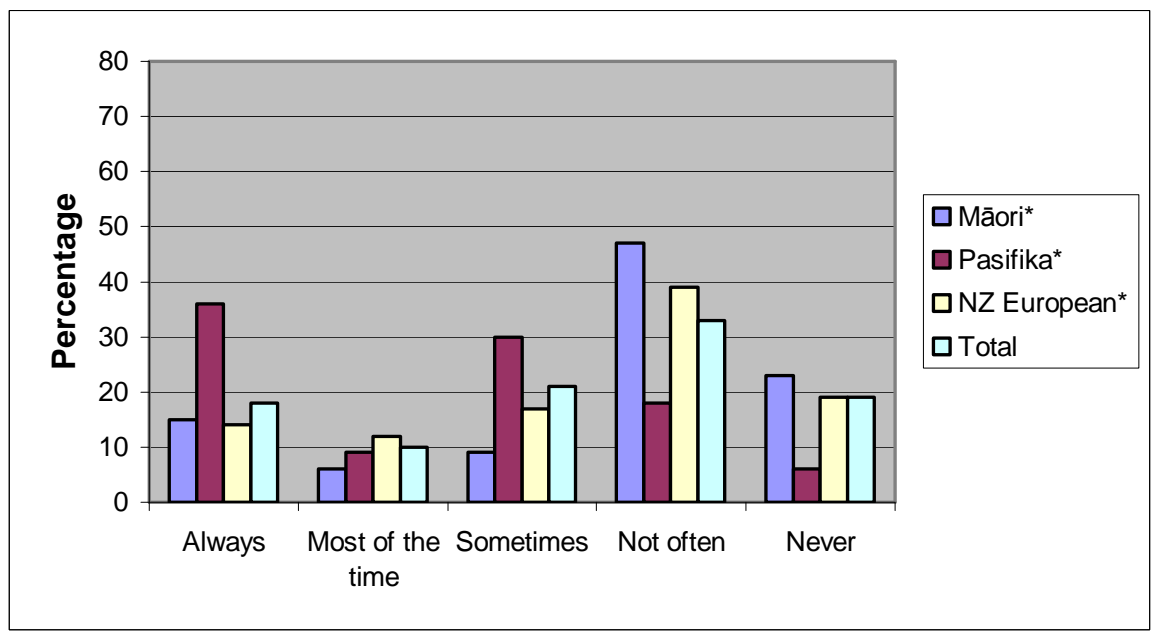

Figure 8.11: Responses to 'I would like my teacher to know about the Pacific world' (\%)

Fairly consistently across ethnic groups, low importance was placed by students on their teacher knowing about the Māori world with combined results for 'not often' and never' of $58 \%$ overall (60\% Māori*, $57 \%$ Pasifika*, 55\% European*) (Figure 8.10$)$. For Māori and European students, the results regarding the importance of their teacher knowing about the Pacific world were similar to knowing about the Māori world. Pasifika* students felt much more strongly than others that their teacher know about the Pasifika world (45\% responding that it was important to them 'always' or 'most of the time') (Figure 8.11).

The Term One data also showed that higher proportions of Māori and Pasifika* students wanted their teacher to know about the Māori and Pacific worlds (20\% and $45 \%$ respectively) than to use such examples in mathematics $(9 \%$ and $28 \%$ respectively). 
Data gathered later in the year showed that most students did not know whether or not their teachers knew about the Māori and Pacific worlds (over 64\% across all groups and over 59\% across all groups respectively) (Figures 8.12 and 8.13 ).

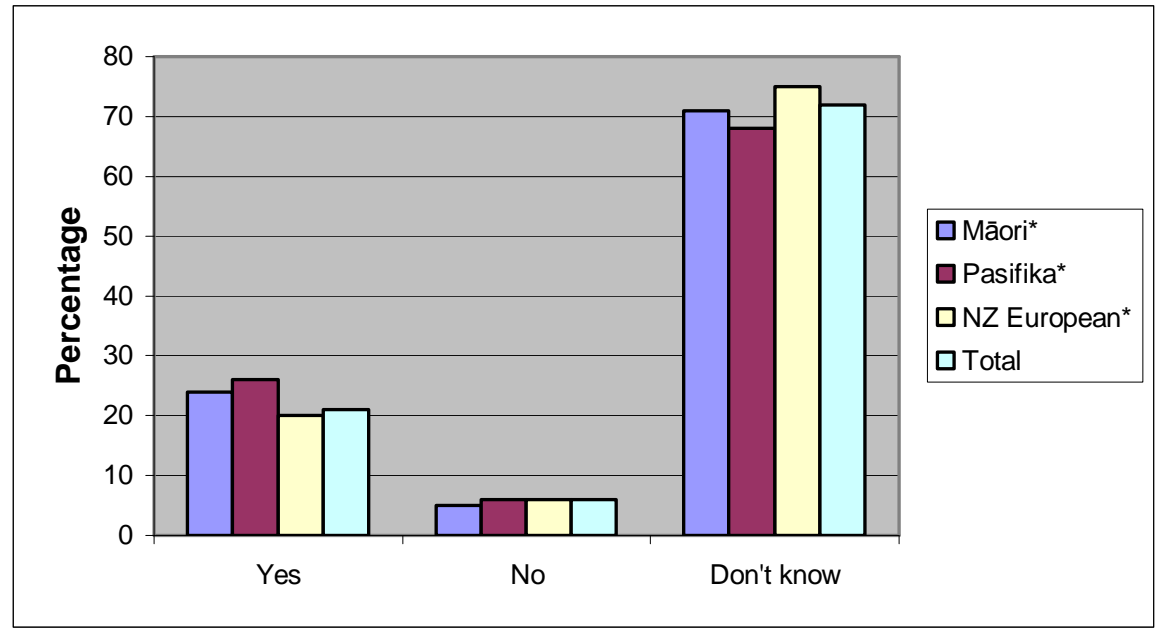

Figure 8.12: Responses to 'My teacher knows about the Māori world' Note: Mean \% for Questionnaires Two and Three

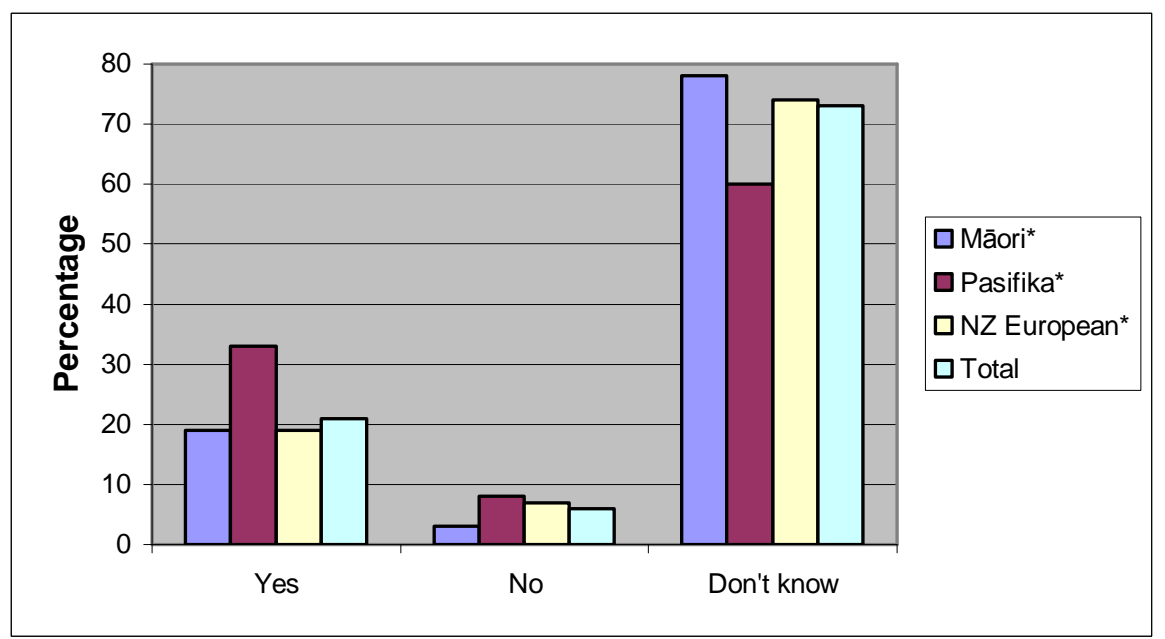

Figure 8.13: Responses to 'My teacher knows about the Pacific world' Note: Mean \% for Questionnaires Two and Three

The results (Figures 8.12 and 8.13 ) provide further evidence that many students do not expect to find links between mathematics and Māori and Pasifika cultures, that teachers may not be displaying their knowledge of these cultures in their teaching, or that when they do, it goes unnoticed by students.

\subsection{Students' perceptions of links between students' cultures and mathematics}

Again, corresponding questions from the three questionnaires were designed to identify any differences between students' desires and teacher practice. Students were asked in Term One to indicate how strongly they agreed with the statement 'I 
would like my teacher to use Māori and Pacific examples in maths' (Figure 8.14). Later in the year they were asked to indicate whether or not their teacher used such examples (Figure 8.15). The results in this section should be interpreted in light of the students' overwhelming lack of experience of such teaching, as indicated by the interview and lesson observation data.

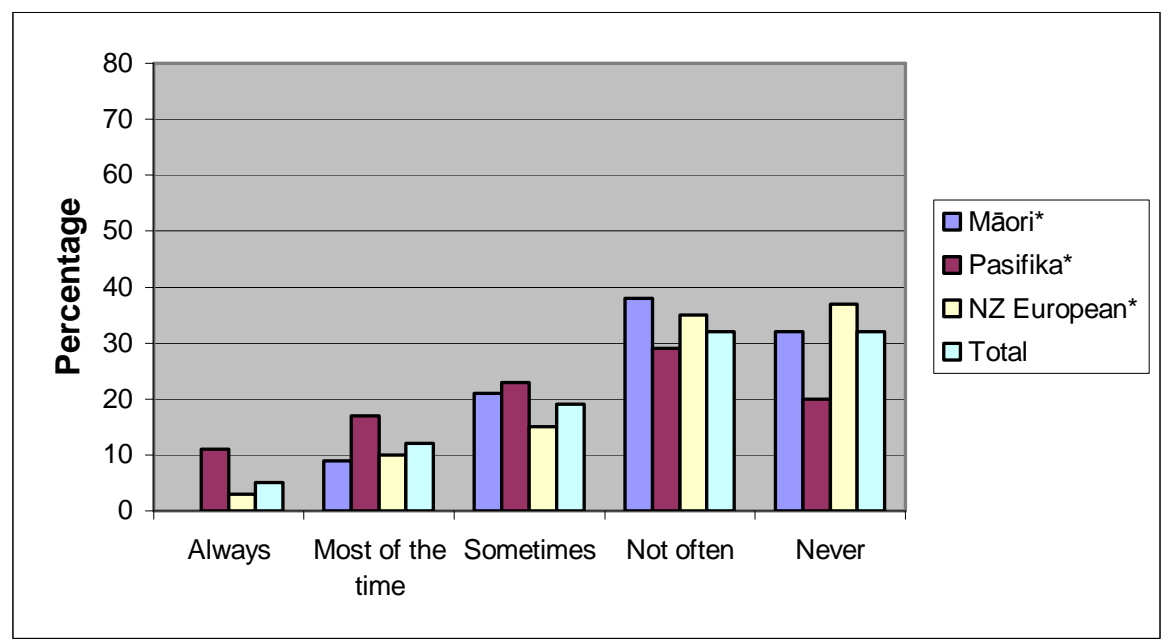

Figure 8.14: Responses to 'I would like my teacher to use Mãori and Pacific examples in maths' (\%)

In Term One most students (64\% overall) indicated they did not want their teacher to use Māori and Pacific examples in mathematics. Of those in favour of such examples being used, Pasifika* students were more favourable than Māori* and European*, with $28 \%$ responding 'always' or 'most of the time' (compared with $9 \%$ and $13 \%$ respectively for the other groups). Responses across ethnic groups were fairly consistent later in the year (Figure 8.15) with high proportions of students (all groups over $41 \%$ ) indicating their teacher did not use such examples. Many students (over $30 \%$ overall), and particularly Māori (53\%), did not know whether or not their teachers used Māori and Pacific examples (Figure 8.15).

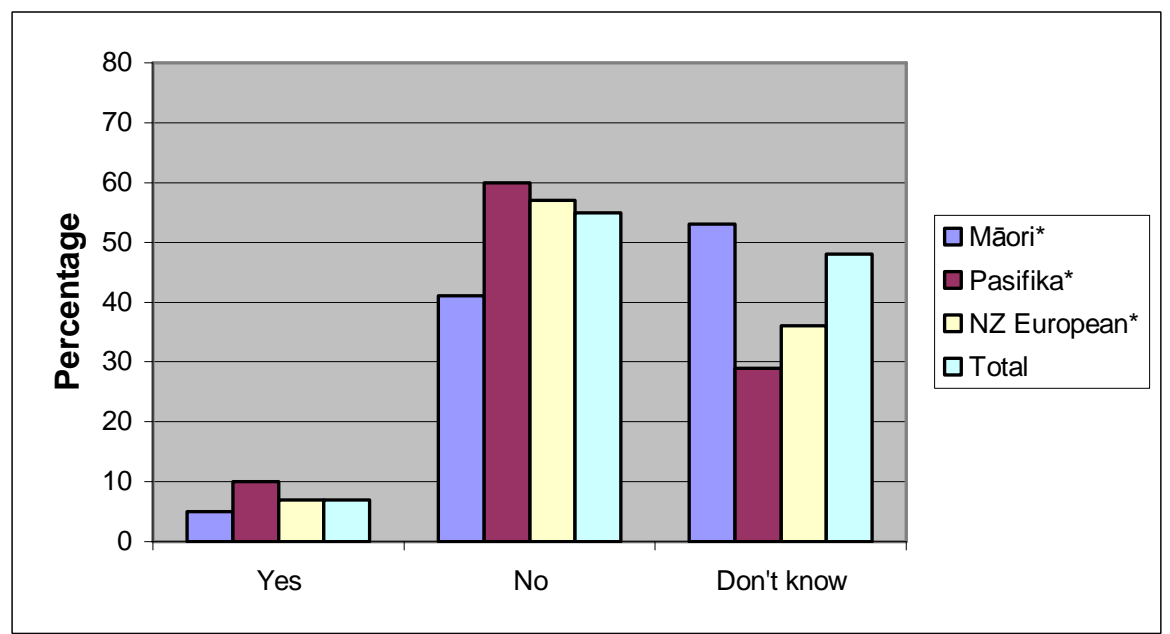

Figure 8.15: Responses to 'My teacher uses Māori and Pacific examples in maths' Note: Mean \% for Questionnaires Two and Three 
A range of views regarding the importance of culturally-based mathematics examples within mathematics learning were discussed in the interviews. Many students claimed little or no experience of this type of mathematics teaching in their current or previous mathematics learning:

So is that something that you're used to happening in maths, teachers bringing in examples from different cultures? Because you've been learning maths from year one all the way through to now? (Interviewer)

No, just one, usually like, European, which is all right cause yeah, I kind of understand it better. (SS student, N)

Two possibilities leading to these claims could be that they had indeed not experienced mathematics teaching in which examples from cultures other than European were used, or that they had, but had not identified or remembered it.

Students often showed some level of surprise during questionnaire and interview data gathering at the inclusion of questions regarding students' cultures; further evidence that they did not expect links to be made between their mathematics learning and the cultures represented in their class and school. Some students thought that it would not be helpful for their learning or for their later life for links to be made between cultures and mathematics:

It would just be the same as normal maths if they added on a bit of culture. (UM, M male and $\mathrm{E}$ male)

But do you think it would help you with learning maths? (Interviewer)

I think it would be better if they keep it how it was. (UM, M male and E male)

OK, just sort of straight maths? (Interviewer)

Yeah. (UM, M male and E male)

Do you think it's important for your maths learning to use examples from people's cultures or that it is not important for your maths learning? (Interviewer)

I don't really know because I don't know whether in the future they'll be using, say for example Māori in maths. I think it's important that you bring the culture into maths but I don't know whether they'll be using Māori maths in the future. (SS, $\mathrm{M}$ female and $\mathrm{N}$ )

OK, so if you see your culture and mathematics, do you see them as things that go together or things that are pretty much separate, or there's a little bit of an overlap? (Interviewer)

Oh. I see them as separate things. (SS, M female and N)

Yeah, 'cause as she said, that Māori maths, we won't ever need that in the future but just maths by itself, we'll definitely need that. (SS, M female and N) 
Again, concerns regarding equity emerged, such as the teacher being biased towards including only some cultures:

If you have examples and they're all to do with, like Pacific type questions, and then people would think, 'oh, why is she just doing that culture? Why not anything else?' But if you do a range of them then that would be all right, but kind of different. (SS student, N)

One interview indicated that despite initially viewing mathematics and culture quite separately, students could quickly imagine the relevance of culturally-linked examples. The following extract shows how one pair of students spontaneously created their own examples of how culturally-based examples could be incorporated in their mathematics learning:

I see it quite different 'cause, like there's your culture and there's maths, so maths is with all these numbers and the teacher, and the culture is with your parents and family, so it's quite different. (WW students, $P$ male and $P$ female)

So you see them as two separate things? (Interviewer)

I reckon you could use culture for, like statistics, like how many people live in Samoa and stuff like that. (WW students, P male and P female)

Or how many children were born there in a certain year and stuff. (WW students, $\mathrm{P}$ male and $\mathrm{P}$ female)

You see I never thought of that. (WW students, $\mathrm{P}$ male and $\mathrm{P}$ female)

And you'd like that to be used? (Interviewer)

Yep. (WW students, P male and P female)

So if there were places where it made sense, it would be a good idea, but if it's just trying to do it when it doesn't really fit, it wouldn't seem very sensible? (Interviewer)

Yeah, but to have true statements that were taken from the [Samoan] census would be good. (WW students, P male and P female)

The use of culturally-linked mathematics learning was important to some students:

Do you think that in maths it would be good to use examples from cultures? (Interviewer)

Yeah, well I am a very cultural person. I like people knowing that I'm not just who they think I am. I've got other cultures in me, but yes, I do think that it would probably be good sometimes if they used other cultural ways of explaining things 'cause there's a whole lot of people in our class who are Samoan and that and they sometimes don't understand what Mr is saying because of the way he says it, like the way he says it in his understanding, and they understand it in a different way, so yeah. So if it comes from a different culture it might help them a bit. (WW student, $\mathrm{M}$ female) 
Some students believed subjects other than mathematics were more closely linked to their culture than mathematics, and that incorporating culture would not help their mathematics learning:

Do you feel for your maths learning it's important that your teacher cares about your cultural identity? (Interviewer)

Well kind of, 'cause I kind of think it is important, but then it may not be because we're there for maths and not for discussion about cultural things, that's for a different classroom. (WW student, M female)

No, focus on the maths and leave other things to other teachers. (WK student, N)

Okay, so just focus on the maths. So are there other subjects where it is important for teachers to know about your culture? (Interviewer)

I would say English and all that because you do more to do with culture. (WK student, N)

Culture doesn't really have anything to do with maths. I don't think my culture or things to do with my culture will help me with my maths. (ST students, E female and $E$ female)

Do you think it helps with any other subjects? (Interviewer)

Mmm, art might have something to do with culture. (ST students, E female and $E$ female)

So most learning at school doesn't have much to do with culture? (Interviewer)

Well some of it does, like all the cultural activities. (ST students, E female and E female)

So do these happen inside the classroom or outside the classroom? (Interviewer)

Well, outside of the classroom, but also in Social Studies there's a lot we are doing about culture. (ST students, E female and E female)

Like if you take a language, then, yeah, it's more about culture, but not necessarily maths or science and that sort of stuff. (UM students, $O$ female and $\mathrm{E}$ male)

OK, so there are no other subjects that use cultures, just language, cultures come into it a bit? (Interviewer)

Probably social studies, 'cause that's like stuff round the world and that. (UM students, $\mathrm{O}$ female and $\mathrm{E}$ male)

But other subjects are pretty culture-free? (Interviewer)

Yeah, almost every subject is culture-free. (UM students, $\mathrm{O}$ female and $\mathrm{E}$ male)

Well if [the class] was social studies then sure, cultures would be important to discuss, but why maths? (SS student, questionnaire response) 
The next interview extract provides another example of a student's quest for equity in teachers' practice, confusions between culture and language, their belief that culture and education are largely separate, and furthermore, that culturally linked examples should be limited to subjects 'less important' than mathematics and English:

It's fifty-fifty cause everybody has a different culture, you can't put learning, put every culture in the learning so it's just, put it into the strong, the majority, 'cause everybody has to learn English. (SS student, E male)

OK and so what about bringing culture into learning in other subjects? Subjects other than maths? (Interviewer)

Yeah a bit, oh I wouldn't really, not English but with other subjects. (SS student, E male)

So bringing cultures into other subjects might be more useful than bringing it into maths? (Interviewer)

Yeah, cause maths and English are the two most important things that you will get in your life and you can't put numbers into different languages and all that. (SS student, E male)

OK, you think it would be more confusing, and not help you learn? (Interviewer)

Yeah, it would be more confusing for people in different cultures to read - a person who was not so fluent, or who was fluent. (SS student, E male)

Other students also thought the question regarding including culturally linked mathematics examples inferred the mathematics teaching would be in another language:

I don't really see how culture would really help mathematics unless it was like in the Samoan language for someone who could only speak Samoan. (UM students, $\mathrm{M}$ male and $\mathrm{E}$ male)

Everyone in the class has different cultures, and some people came from the Islands last year and they don't know everything about maths so it's sometimes is easier to [explain it in Samoan], cause there's like quite a few people that understand full Samoan and that will talk in Samoan, so it would help them. (SS student, N)

Well it depends really, 'cause some people might understand Samoan, some people might not, some people might understand Māori, some people might not. (WW student, E female)

The importance of the teacher knowing enough about language and culture to appropriately use culturally related knowledge in the classroom was highlighted:

Do you think it is important to have examples from your culture used in the Maths classroom? (Interviewer)

It just depends on what you use my culture in. (WW students, $M$ male and $P$ male) 
Yeah, it just depends on what you use my culture in, like if you're going to use an example in the language you may as well speak it properly because like if they're going to describe something in the maths you know, they should get it right. (WW students, $\mathrm{M}$ male and $\mathrm{P}$ male)

So it's a matter of doing it properly if you're going to do it? (Interviewer)

Yeah. (WW students, M male and P male)

In summary, many students did not want Māori or Pasifika examples used in mathematics, but over a third were unsure whether or not their teachers used such examples. Many students viewed mathematics and culture as separate and most could not readily identify any benefits from integrating cultural knowledge and mathematics instruction.

\subsection{Discussion}

Their heritage cultures were important to students across the board although evidence from some New Zealand European students in particular suggested they may lack understanding of what their culture encompasses. In contrast a range of specific aspects of students' cultures were identified by $27 \%$ of students who responded to Questionnaire Three.

High proportions of students did not know whether or not students' cultures were important to their school (40\%) or to their teachers (61\%). Even given the degree of students' confusion regarding cultural identity indicated by other sections of the data, this seems alarming in multicultural schools in a multicultural community and country.

Very mixed views were expressed (including both extremes) regarding the importance and relevance of students' culture to their maths learning. There was also a range of opinions in each study class about whether or not cultures are seen as important at their school and by their teacher and whether or not their culture is relevant at school in general. Students expressed they had little experience of mathematics learning being linked to Māori or Pacific Nations contexts and most did not know about their teachers' knowledge of the Māori and Pacific worlds.

Current educational research and policy documents discuss the importance of education enhancing students' feelings of cultural identity (e.g., Ministry of Education, 2006c, 2008c), but practices consistent with this are not apparent in the study data (classroom observations, student results or teacher results). 
The analysis of the study data shows the value of investigating these questions. Further work is needed to understand more clearly the determinants of students' beliefs regarding what constitutes their culture, the relevance of culture to mathematics learning, how students feel about their cultures being reflected at school and in mathematics learning and the reasons for these feelings, and the place of culturally related contexts as mathematics learning experiences. It is reasonable to suppose that if one believes that one's culture is important, then it is also important for learning, and vice versa; if one believes that one's culture is inconsequential then perhaps cultural emphasis may be or may be seen to be a barrier to learning. People who never think about culture may not have a belief either way, and cultural emphasis may have a neutral effect on their learning. This hypothesis could explain the wide spectrum of answers, and the possibility of further studies coming to contradictory conclusions.

Returning to the whāriki model (Figures 3.5 and 6.1), the results in this chapter highlight that knowing each other's cultures and enhancing feelings of cultural identity are needed for all students to enrich and acknowledge those whose culture is important to them and to help those who have little engagement with issues of culture to develop understanding of their own culture and that of others. Challenges to implementation of such practice are likely to include students' apparent lack of experience of mathematics teaching linked to their cultural backgrounds and their varied perceptions regarding the relevance of culture to mathematics. 


\section{CHAPTER NINE}

\section{Classroom Practices that Foster a Caring Environment}

This chapter highlights the themes that emerged across data gathering methods regarding practices that support a caring classroom environment: one-to-one teacher-student interactions; teachers sharing their personal identities and making opportunities for students to share theirs; respect; and use of humour (Section 9.1). The chapter then returns to the integrated 'teacher care mathematics whare' model in order to summarise the interplay between the typologies described in Chapters Five to Eight (Section 9.2).

\subsection{Practices that support caring classrooms}

The elements of classroom practice discussed in this section are inter-linked and mutually supportive. For example, teachers privileging time for one-to-one interactions can make students feel they are known and respected and can increase teachers' and students' comfort in sharing themselves including by means of humour. Each of the elements in this section has emerged in several analysis chapters, indicating that these behaviours are likely to contribute in a fundamental way to the development of caring classroom environments, and thus to effective learning.

For each element, the main findings across data gathering methods will be described, as will the extent to which study teachers used the practice, factors limiting the practice, and opportunities to enhance the element in classroom teaching.

\subsubsection{One-to-one teacher-student interactions}

The international literature (e.g., Noblit et al., 1995), New Zealand literature (e.g., Hawk \& Hill, 2000; Ministry of Education, 2007c) and the classroom trials of data gathering tools (Section 3.2.2) indicated the importance of exploring the contribution of one-to-one teacher-student interactions to teacher care. Teachers' use of one-toone interactions was common across all study classes and the possible effects of these on the development and display of teacher care emerged in all analysis chapters. 
The teachers who exhibited the greatest levels of care (Chapter Five) for their students and whose classrooms were most focused on learning (SS, WW, WK, UM) created more opportunities than the other teachers for one-to-one teacher-student interactions. Each of these teachers interacted with every student multiple times for multiple purposes during all lessons. The interactions appeared to be founded on the expectations that students would respond, be engaged with their work, and make efforts to learn. Students arrived at these classes knowing they would be expected to answer questions about their work and that they would be treated as individuals:

He always asks, always makes us ask questions, and he's always trying to get us into the work. (WW student, male)

In these teachers' classrooms, the one-to-one interactions focused on students as individuals tended to occur at the beginning and end of lessons, with a few instances during the main body of the lesson. Each of these teachers made multiple opportunities for one-to-one interaction focused on mathematics learning and progress during the body of the lesson (e.g., collecting responses to questions for reviewed and new work, moving around the room to check students' work books for content and presentation, answering students' questions, and monitoring and giving feedback on progress). The importance for students' learning of one-to-one teacherstudent interactions is highlighted by one student's comment:

It's easier for me when he explains something when he's standing right next to you, talking just to you. (WW student, male)

In contrast, the teachers who appeared to have the least caring classrooms (UU, ST) infrequently interacted with students by name, for mathematical learning or purposes other than mathematics learning, and the one-to-one interactions that did occur in these classrooms appeared to have limited impact on student engagement, affect, and learning (Chapter Five). Seemingly low teacher expectations, mirrored by students, regarding the quantity and quality of students' mathematics engagement and progress, appeared to be linked with low levels of one-to-one teacher-student interaction.

\subsubsection{Making opportunities for sharing personal identities}

Clark (1995b) determined that Pasifika students at a New Zealand university had increased engagement and achievement in learning environments they described as 'comfortable'. In her later study into British secondary school students' perceptions of what made for a comfortable classroom, Clark (1997) found that students 
preferred problems relevant to their interests and a more social classroom style and greater individual attention than offered by traditional approaches. Furthermore, when students felt the 'real them' was different to their classroom persona, they were less likely to continue with mathematics learning.

The findings of this study build on Clark's (1995b, 1997) work, providing evidence that effective and focused one-to-one teacher-student interactions which can help students and teachers feel comfortable in sharing of, and being, themselves in mathematics lessons are linked with mathematically productive lessons. Examples include that the lesson observations of classes with the study teachers who appeared to most value their students as individuals and individual learners showed higher levels than other observations of mathematical progress, on-task time, student-initiated interactions, and student and teacher enjoyment.

Most study teachers reported getting to know about their students in informal and rather ad hoc opportunistic ways, for example through one-to-one spur of the moment conversations as the year progressed. This study indicates that many students want their teacher to know about them as people and that teacher knowledge about their students and what students would like their teacher to know about them do not always match well. Results from this study indicate that formal ways for teachers and students getting to know each other, such as the letter-writing task used by SS and UU, could be useful tools for all teachers to use to create a better match between what students want their teachers to know and what teachers do know, in turn enhancing students' classroom comfort, and their mathematics learning.

Pedagogies used by the study teachers that appeared to create opportunities for them and their students to reflect personal identities in mathematics lessons included using games, competitions, linking learning tasks to their own and students' lived realities, group tasks, and encouraging students to discuss their work with others (Chapter Five).

Factors that appeared to create classroom climates that limited expression of teachers' and students' personal identities included negatively framed disciplinary interactions, mistrust, and lack of class cohesion. 


\subsubsection{Respect}

Respect was not initially a major focus area of this study. However, the theme of respect was present in the literature on teacher care (e.g., Andersen, 1995; Elias et al., 1997; Haynes et al., 2003; Noddings, 1992) and the importance of respect as a component of teacher care was a recurrent theme in student and teacher data in all analysis chapters. Furthermore, examining teachers' and students' views of respect is timely given the inclusion of 'respect' as one of the core 'values' of the New Zealand curriculum (Ministry of Education, 2007a; Section 1.1) and the emphasis given to respect in other guiding documents for New Zealand education (e.g., AltonLee, 2003; Ministry of Education, 2008c).

The students' respect for the teacher, the teacher's respect for the students, mutual respect between teachers and students, and self-respect all featured in the data. Teacher respect was discussed in terms of respect for cultures, for views, and for student decision making, particularly in terms of teacher understanding regarding contexts that may contribute to aberrant student behaviour. Student and teacher views of the importance of respect emphasised that respect assists with:

- teachers understanding their students' behaviour;

Part of my job is training people to be Maths teachers, what do you think is the most important thing that I should tell them about teaching Maths in high school? (Interviewer)

Teach them to respect the kids. Even if they're naughty, they could have reasons, so if they're naughty you've just got to pull them aside from the other students and ask why you're like this or is there anything wrong at home or at school or something. (WW student, male)

- student-centred teaching;

How do you know that your maths teacher cares about who you are? (Interviewer)

He treats us with respect. He doesn't do like some teachers on TV and in the movies. They just say 'just do your work'. (WW student)

Your teacher can know about who you are but do you think that it's important that you feel that they also care about you as a person? (Interviewer)

Yeah, they should respect you, and should care about who you are. (WK student, male)

- fostering learning;

What do you think is important for students to be like to achieve well in maths? (Interviewer)

You need to respect your teacher, so that you can listen and learn properly, because if you are being disruptive, you disrupt the teacher and the whole class as well as your own learning. (ST student, female) 
Some of the students that are more secure in themselves don't really care much about the teacher because they'll learn in spite of you, but if a student is feeling insecure they need to know that you respect and appreciate them as a person. And once they've built a relationship with you then they feel comfortable to try things that they didn't feel like they knew before. They're more inclined to take risks. (UU)

- developing mutual respect between students and teachers;

Do you think it's important to show that you care about who the students are as people? (Interviewer)

Yes, indeed. You have to respect them, otherwise they won't respect you. Respect is important, I value it a lot and I enjoy working with students who show respect, even to themselves. If they don't respect themselves they can't do anything in the school. (UM)

- creating a caring environment; and

Do you think he cares about who you are? (Interviewer)

Yes. (UM student)

And how do you know that? (Interviewer)

He respects us. (UM student)

How does he show that? (Interviewer)

He treats everyone the same. (UM student)

- showing acceptance of students' cultural identities.

I don't think it's always vital [to know about students' cultural identities] because all cultures have the same basic needs, in terms that they want to be respected, they need to know that they belong and that you care about them as a person. That seems to go right across all the cultures that l've come across through the years. So while it's important to respect it I don't think you need to know every single thing about their culture. (UU)

The data indicated that study teachers generally displayed respectful behaviours towards students (e.g., greeting students as they arrived at class, responding to students' requests in a timely manner) and that teacher respect helped to engender student respect. Some evidence of less respectful teacher practice was evident (e.g., making a class wait outside the classroom for ten minutes without explanation, public admonishment) but infrequent. Less respectful teacher behaviours may have been the result of teacher stress, challenging student behaviour, or teachers not realising how their actions may affect students. The study findings indicate that teacher-student respect in classroom teaching can be enhanced by a mutually held clear focus on mathematics learning and knowledge of one another as people.

The study findings add to the literature on teacher care (e.g., Andersen, 1995; Elias et al., 1997; Haynes et al., 2003; Noddings, 1992) by indicating the important roles respect plays in fostering effective teacher-student relationships. Consistent with Alton-Lee's (2003) synthesis of 'best evidence' regarding effective learning of diverse 
learners, the theme of respect emerged strongly in the students' responses regarding teacher care for students' cultures (Chapter Eight). This research suggests that more needs to be understood about teacher respect, for example, the impact of teacher respect on student motivation and achievement, how mutual respect can be nurtured, and how students recognise and perceive their teacher's respect for them.

\subsubsection{Humour}

Study results indicate that teachers can be strategic regarding both their own use of humour and their responses to the humour students offer to improve teacherstudent relationships and enhance learning opportunities (Sections 5.1.1, 5.2.3, 5.3, 6.2, 7.1.1). Humour also featured in the literature used to inform this study (e.g., Hawk \& Hill, 2000; McCabe, 1995; Ministry of Education, 2007c; Powell, 2000) and in cultural advice as a valuable tool that can lighten the atmosphere of a classroom, can help save face, and can increase engagement and motivation.

Observational and interview data in particular indicated that many students appreciated teacher humour. At times a light-hearted response to behavioural issues and student humour may have provided better outcomes for individuals and for the classroom atmosphere than those resulting from disciplinary actions. For example, on several occasions ST responded to a particular student's humour with a sharp reprimand and by sending the student out of the classroom. This appeared to result in the student being shamed, missing the classroom instruction, and assuming negative attitudes towards the teacher and the subject. In the observer's opinion, the student had in each case offered the humour as a means of assisting the teacher deal with a tense classroom management issue. A teacher response with humour in kind, followed by refocusing the students back on to the lesson, would have been a more positive response in my judgement. Instead the opportunities were lost with concomitant negative consequences for the class in terms of deterioration of the classroom atmosphere; for the student, in lost learning opportunities; and for the teacher, in reduced student trust and reduced teaching effectiveness.

Three of the study teachers used or responded to humour at least several times each lesson (SS, WK, UM) and the others used humour less frequently or not at all. Factors that appeared to limit teachers' use of humour were the lack of a quality teacher-whole class relationship, teacher stress, and teachers' personal senses of humour. There appeared to be many opportunities for teachers to include or respond to student humour in all lessons. 
The strategic use of humour is seldom deeply explored in teacher education. The findings of this study suggest that humour is a valuable teaching device that affords opportunities for nourishing teacher-student relationships and enhancing learning, and is certainly worthy of increased attention.

\subsection{The Teacher Care Whare}

The purpose of the whare (Figure 9.1) is mathematics learning. The teacher and the students are in the whare together to achieve mathematics learning for the students. The teacher-student relationships and care for students stem from, and are bound up with, this purpose. By and large, it is within the three-dimensional space created within the whare walls, on the whāriki, and in front of the whiteboard that teacher care is displayed and teacher-student relationships enhanced.

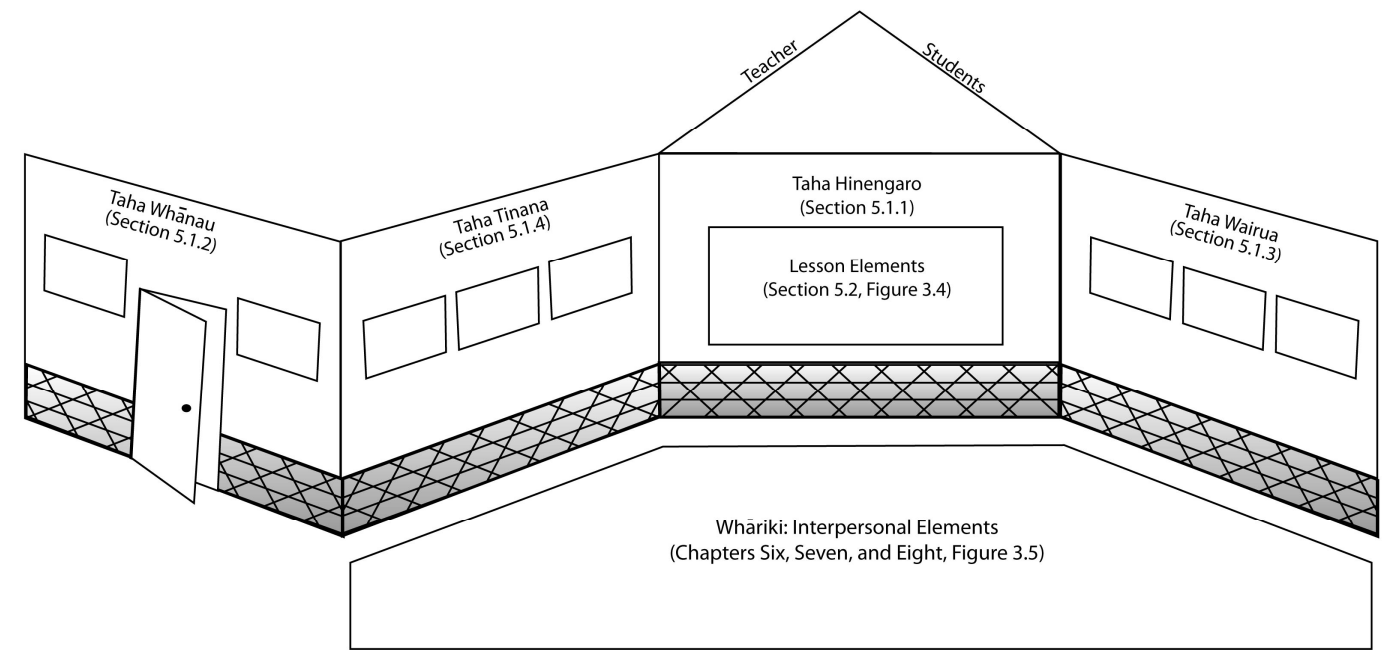

Figure 9.1: The Teacher Care Mathematics Whare

The whare model allows consideration and examination of a wide scope of mathematics teachers' behaviours conducive to generating caring classroom relations. The four walls (Section 5.1), the whiteboard (Section 5.2), and whāriki (Chapters Six-Eight) each remind us in different ways of the importance of attending to our students as people, people who have individual personal and mathematical needs, both of which must be addressed to maximise mathematics learning.

The model can be seen to be idealistic and future-oriented. The many windows can be used to remind us that the mathematics we are learning is for a purpose, to enhance our understanding of, and ability to function, in our world, and all that means to each individual. The door is open, allowing space for the students and teacher to enter and exit, and for parents, school, and community to share in decisions relating to students' mathematics learning. In this study very few instances 
of family or community involvement in students' mathematics learning emerged. Notable exceptions included several students being helped with their mathematics learning by older brothers or sisters, one teacher (UU) sending letters acknowledging students' good work to their parents, and another (SS) contacting parents when she had serious concerns regarding students' progress. These strategies provided a few parents with some information regarding their students' progress but did not involve parents, families, or communities in any substantial or ongoing way. Although it was not the intention of this study to focus on such practices, over all data gathering, very few instances of teachers or students involving parents, family, or community at earlier stages of mathematics learning (e.g., planning, lessons, assistance) emerged.

The study data indicated that when elements of the whare are working well together (e.g., students' well-being, their mathematics learning, attitudinal and dispositional characteristics), effective teacher-student relationships are in place and reciprocity between student behaviour and teacher behaviour exists. Elias et al. (1997), consistent with Lave and Wenger's (1991) concept of a learning environment as a community of practice, refer to the necessary reciprocity between the carer and the cared for. Reciprocity between student and teacher behaviours found in this study included:

- $\quad$ showing and receiving respect (Section 9.1.3);

- students were likely to respond with and initiate humorous or light-hearted interactions with the teacher in classrooms in which the teacher used humour; and

- students were more likely than those in other classrooms to be understanding of teacher behaviours and mistakes in classrooms in which teachers explained their practice and showed tolerance.

According to the principles articulated by Elias et al. (1997) and Lave and Wenger (1991), mathematics classrooms consistent with the whare model are likely to be happy and industrious. Through teacher attention to the four elements of students' well-being (hinengaro, tinana, whānau, wairua, Section 5.1), they will be places that have a clear focus on mathematical learning. They will have energy, positive personal interactions initiated by students and teachers, laughter, movement, and mathematical challenge. They will be places in which students and the teacher are able to be themselves, consistent with Bosworth (1995) and Peterson's (1992) views of teacher care and caring interactions (Section 2.3). They are the learning spaces 
most likely to approach achieving Manu'atu's (2000) vision of 'mālie' within learning contexts (Section 1.2), i.e., the uplifting, energising atmosphere felt through connections between, and commitment to, acts of performance, communication, and the human psyche and spirit.

Finally, the teacher care mathematics whare must be a place where students and the teacher want to be. Comments from two teachers and one student illustrate the fundamental importance for teacher effectiveness of enjoying teaching and wanting to teach:

To teach well you've got to want to teach. (WW)

If you try to do something that you don't like in teaching you get in a mess because you can't do it. You can't do it to a high standard. You can do it only for the sake of doing it but that's not teaching. (UM)

Enjoy your job and enjoy our company. (SS student)

The purpose of the whare is mathematics learning. Teacher behaviours identified through this study as being conducive to nurturing caring teacher-student relationships and interactions appeared to be quickly undermined when the ultimate purpose of being together in the whare was, in the students' minds, not being realised (Chapter Five). 


\section{CHAPTER TEN}

\section{Discussion and Conclusion}

The goal of this research was to examine how teacher care is shown and experienced in junior secondary school multicultural mathematics classrooms. Three areas of care were considered: teacher care for students as individuals; teacher care for students' mathematical progress; and teacher care for students as culturally located individuals. The research participants included six teachers and their classes from three mid to low decile multicultural city schools. The study data included lesson observations, teacher and student interviews, and teacher and student questionnaires carried out at three points during one academic year for each class. An integrated model incorporating three frameworks was used to analyse and describe the results.

This chapter describes issues affecting the research process and results (Section 10.1), the main findings of the research in light of the literature on teacher care and culturally responsive practice (Section 10.2), implications of the findings for teachers, teacher educators, and policy makers (Section 10.3), topics worthy of further exploration that emerged as a result of this study (Section 10.4), and final concluding remarks (Section 10.5).

\subsection{Issues affecting the research process and results}

Considerable care was taken in the design and execution of this study to ensure the data collected were representative and reliable. Cultural and student advisors and teacher experts were consulted, data gathering tools were trialled and refined, and negotiation of all data gathering practicalities was carried out with research participants (Chapter Three). Many lesson observations (around 100 altogether, including between 15 and 20 lessons for each class and teacher) and student interviews were carried out to help ensure representative data samples reflecting the full range of practice and beliefs. Even so, this is only a sample of each teacher's lessons with this class over the entire year and of students' views. All research studies are susceptible to limitations due to the possible effects on findings of the study design, the study sample, non-response error, the data gathering methods and timing of these, and analysis methods (Patton, 2002; Swift, 2002). The effects of each of these on the study findings will be considered in turn. 
This study combined quantitative and qualitative methods and data to explore the breadth of student and teacher perceptions and to allow comparisons. The analysis chapters (Chapters Five-Eight) provide summaries and quotes to illustrate the range of student and teacher views alongside graphs and relative frequencies to illustrate comparisons of perceptions and beliefs across ethnic groups. Although mixed methods research has recently become widely used in education-based research, inherent assumptions grounding qualitative and quantitative methods and interpretation of data from these methods vary and can be seen to be in conflict (Tashakkori \& Teddlie, 2003). Possible effects of the paradigmatic assumptions of the two methodology styles on the reliability of this study and its findings were minimised by:

- $\quad$ using several triangulation techniques (Chapter Three);

- consulting with cultural advisors on data gathering tools, data, and interpretation of the data throughout the duration of the study; and

- identifying the data sources used for each section of the analysis.

Areas of convergence between the results from qualitative and quantitative methods can give greater confidence in findings whilst areas of divergence offer opportunities for new questions (Patton, 2002). In this study, results pertinent to the first two themes (teacher care for students as individuals, and care for their mathematics learning) were fairly consistent across data gathering methods and tools, particularly for the teachers who had the most effective teacher-student relationships in place and were consistently focused on their students' mathematics learning (SS, WK). Variation between interview and observation data occurred for the teachers with comparatively mundane classroom relationships and teaching practice (UU, ST). Differences tended to indicate that teachers' perceptions of their motivations and practice were, in the students' and researcher's views, not consistently exhibited in their classroom practice.

Most areas of divergence in the study findings overall emerged within the theme of teacher care for students as culturally located individuals (Chapters Six and Eight). Possible reasons for less consistency across the data gathered for this part of the study include:

- the limited opportunities to trial the relevant section of the observation schedule;

- comparatively little observational data was collected in this area; 
- many participants' apparent confusion regarding their cultural identity and cultural identity in general, and links between this and mathematics teaching and learning; and

- students' and teachers' apparent lack of previous experience of culturally responsive mathematics teaching.

In order to provide data, analysis, and findings relating to effective practice of teacher care, the study teachers were chosen, in part, for their views that teacherstudent relationships are important for students' learning. Furthermore, three of the six study teachers held positions of pastoral care for the study class (SS and WW were deans and WK the form teacher). In order to focus on the students most likely to progress with mathematics into the senior secondary school and most able to reflect on and describe factors impacting on their mathematics learning, four of the six study classes were made up of students with average to above average mathematical ability. The study sample therefore is unlikely to be representative of all mathematics teachers and students.

Not all students in the study classes participated in the study:

- 22 (out of 161) did not return the consent forms;

- several were absent from each class for each round of questionnaires and interviews; and

- a small number of questionnaire responses were missing or fell between categories (e.g., between Likert scale descriptors).

Furthermore, a large sample (a mix of student self-selected and teacher-selected) but not all students were interviewed. The study teachers reported that the students who did not consent to participate tended to be those less able and less committed to learning. As the study was targeted at the study schools' middle to higher achieving students the impact of the lack of participation of those without consent is likely to be low. No patterns of lack of consent were noted across ethnic groups.

Attempts were made through their teachers to gather questionnaires from students absent when the questionnaires were completed (e.g., questionnaires and selfaddressed stamped envelopes were left with teachers for return). However, very few further questionnaires were gained. In all cases, all collected data were used for analysis and for the quantitative data, the very small number of non-responses were ignored. 
For questionnaire responses recorded between descriptors (e.g., a response halfway between 'very likely' and 'likely'), the descriptor closest to the neutral position was entered into SPSS in order not to overstate participants' responses (i.e., a response halfway between 'very likely' and 'likely' was entered as 'likely').

Other limitations to the findings of the study include:

- "reactivity" (Boulton \& Hammersley, 2006, p. 244), i.e., the researcher's presence may have affected what was observed and researcher decision making (e.g., the selection and description of what was observed and what questions were asked); and

- participants' responses are a very small sample of their "truthspace" (Onwuegbuzie \& Teddlie, 2003, p. 369) and may describe their idealistic thoughts rather than real or likely practice.

The research process (repeated data collection from each study teacher and class) and timeframe (one year of study design and two years of data collection) provided time for reflection and consultation allowing close examination and re-examination of the research questions, data gathering methods and tools, and data gathered. The integrated analysis model provided useful inter-related frameworks for scrutinising the study data, drawing conclusions, and discussing findings.

The lesson observations from the most effective and caring teachers and the student data were most useful in answering the research questions because:

- of the quantity and quality of the quantitative and qualitative data gathered from these sources;

- of all the data gathered, these data sources most closely matched the study focus; and

- the teacher interview and questionnaire data were most likely to be susceptible to differences between espoused beliefs and actual practice (Onwuegbuzie \& Teddlie, 2003).

The data from the least effective study teachers (although subsequently useful for drawing comparisons between more caring and less caring practices, see Chapter Five) tended to have less internal consistency and lessons observed with these teachers tended to focus more on discipline and control than on caring teaching and learning practices. 
The study design led to the first student data collection occurring after the researcher and students had shared more than ten mathematics lessons. Subsequent student data collection occurred after even greater amounts of shared time and experience. Thus the study students felt some knowledge of the study and the researcher before data were collected from them and knew their opinions would be valued. There is little doubt that the researcher-participant proximity prior to data collection enhanced students' trust in the researcher and her intentions, and resulted in honest, open, and quality questionnaire and interview responses.

If conducting a similar study in the future, the following changes would allow further reliability and trustworthiness in the findings:

- working in greater partnership with cultural co-researchers of study participants' ethnicities than allowed by the nature of PhD study;

- aiming to reduce the number of students who did not give consent to participate in the study; and

- if necessary, changing study sites, to maximise the suitability of all study teachers.

\subsection{Main study findings}

The study achieved its aims. The findings provide New Zealand mathematics teachers with strategies they can use with confidence to develop caring classroom relations that will meet the desires and needs of many New Zealand students, including Māori and Pasifika, and will assist towards maximising mathematical learning opportunities. The analysis has shed clear light on:

- what students would like their teachers to know about them and what they would like to know about their teachers (Chapter Seven);

- how students wish to be treated by their teacher during mathematics lessons (Chapter Seven);

- the importance placed by students and their families on their personal mathematical achievement and growth (Chapter Seven);

- strategies that will assist in implementing the new curriculum in mathematics learning, in particular the key competency 'relating to others' (Chapters FiveEight);

- the increasing complexity of the cultural diversity of New Zealand's secondary school students (Chapter Four); 
- the apparent lack of depth of understanding regarding cultural identity and how this could relate to mathematics learning (Chapters Six and Eight); and

- the seeming lack of family and community involvement in students' mathematics learning at the junior secondary school level (Chapter Nine).

Bishop et al. (2003) showed that increased teacher effectiveness and increased motivation and learning for Māori were results of teacher professional development based on the "effective teaching profile" (Bishop et al., pp. 95-116). This study further informs understanding of the elements of the effective teaching profile (and of additions to the profile that emerged through this study) for students in low socioeconomic multicultural mathematics classrooms (Chapter Five).

The literature (e.g., Gay, 2000; Noblit et al., 1995; Noddings, 1992, 1993, 1995, 2003, and others, see Chapter Two) presents a strong case for enhancing students' learning of effective teacher-student relationships and of attending to care for students and their learning. The study has helped to respond to calls (e.g., Ferguson et al., 2008; Nuthall, 1999; Teven \& McCroskey, 1996) to identify ways in which teachers can show care to their students and has added to the literature by indicating:

- which teacher behaviours from the literature are most salient for New Zealand students similar to the study participants (Chapter Five-Nine);

- precursive teacher behaviours (e.g., showing respect, using one-to-one interactions, responding with humour (Chapter Nine)) necessary for elements from the literature on teacher care such as empathy, understanding, learnercentred practices (Section 2.3.3) to be achieved; and

- through the study's observational data, consistency between New Zealand classrooms similar to those in the study and Noblit et al.'s (1995) belief that acts of caring directed towards individuals can have a carry-over effect to others in the class.

The findings indicate that many of the key messages from international literature regarding teacher-student relationships and teacher care (Chapter Two) are also applicable to New Zealand secondary school mathematics teaching. This research contributes to teacher care theory by:

- providing an example of a classroom observation schedule which amalgamates a wide range of teacher practices distilled from the literature, and modified or confirmed through cultural consultation, that help New Zealand mathematics 
teachers exhibit care for students, their mathematics learning, and their cultural identities; and

- integrating key ideas from the literature and existing typologies with the study findings into a new model for teacher care, the Teacher Care Mathematics Whare.

Three existing typologies (Averill, Te Maro et al., 2009; Bishop et al., 2003; Durie, 1998) provided sound bases for development to help describe the results of this study. The developed typologies (Chapters Five-Eight), integrated into a new holistic model (Figures 3.3, 9.1 and Chapters Five-Nine), acknowledge students' holistic health and well-being, their dispositions to mathematics learning, and culturally responsive teaching practices. The Teacher Care Mathematics Whare, can be used to describe, develop, and analyse caring mathematics classroom practice. It is hoped that elements of the model can be used to enhance the mathematical learning opportunities of New Zealand students. The bicultural flavour of the model enhances its suitability for New Zealand classrooms, in which teachers are expected to demonstrate knowledge and understanding of the implications of the principles of the Treaty of Waitangi, and of te reo me tikanga Māori (Ministry of Education, 1998).

Few bicultural models for mathematics teaching and learning exist. The whare model emerged from this study into teacher care in mathematics classrooms. However, many aspects of the model are sufficiently generic that it seems likely that it could have transferability within New Zealand to other curriculum areas and other year levels. It is hoped that this study can also make a contribution to the current international movement towards exploration into culturally responsive mathematics teaching. While the whare model is linked with Māori cultural elements, it seems possible that similar models, necessarily created with cultural consultation at all stages, could be developed in other countries to draw from their societies' cultures to illuminate teacher care for students, their cultural identities, and their mathematics learning in ways responsive to the cultures present.

Many students find learning mathematics challenging. To some extent, many societies often perpetuate images of mathematics as a difficult and inaccessible subject. The findings from this study indicate that many students appreciate what they perceive as a personal commitment from their teacher towards their learning as individuals and as a class. 
While this study sought to explore three aspects of teacher care, overall reflections on the data and findings include that:

- each of the study teachers demonstrated each area of teacher care (for the student as a person, a culturally located person, and for their mathematics learning) to differing extents;

- teacher care for students' mathematics learning was important to many students;

- teacher care for students as individuals was important to many students;

- many students were unaware whether or not their teachers were showing care for them as culturally located individuals; and

- students felt care in a holistic way, and as long as they were able to learn well, they were tolerant of their teacher's minor lapses in the three areas of teacher care.

There are many ways in which the teachers in this study showed care for their students' learning. Examples included teachers being organised, providing clear goals and feedback, being explicit about their teaching decisions and the rationale for these, acknowledging and celebrating success, keeping students on task, and providing encouragement when students were having difficulty. These effective teaching practices appeared to assist in developing strong teacher-student relationships because they helped show students the teacher's care for their learning.

Teachers showed their care for students as individuals by knowing and using their names, knowing about them as people, acknowledging them (e.g., greeting students by name as they arrived in class), showing empathy and responding to needs, and having one-to-one interactions about things other than mathematics learning.

Care for students as culturally located individuals was shown by acknowledging and showing respect for students' cultures, showing flexibility when demands from students' homes, sporting commitments, and cultural performance groups affected their work, and using words from students' heritage languages.

The study findings suggest that teacher care for mathematical learning is necessary for teacher-student relationships that lead to effective learning. Teachers' respect for students, their time spent assisting students one-to-one, their use of humour, their knowledge of students as individuals, and opportunities for display of personal identities 
are all important contributors towards effective relationships. However, the findings of this study suggest they are secondary to teachers' care for students' learning. If teacher care for learning is not evident, teacher-student relationships appear to be undermined. That is to say, the most important thing mathematics teachers should be attending to is how they show they care for their students' learning.

\subsection{Implications of the findings}

The study findings indicate that some mathematics teachers are already using practices consistent with Ministry of Education policy for Māori learners' education (Ministry of Education, 2008c). Many of the study teachers knew, respected, and valued their students and shared with them some of the responsibility for learning. However, the results indicate that much work by teachers and teacher educators is needed to fully realise the aims of Ka Hikitia (Ministry of Education, 2008c) (such as to ensure Māori can achieve 'as Māori') and implement practices consistent with those described by the Education Review Office (2002) (e.g., incorporating elements of Māori language, culture and knowledge into the curriculum).

Over half of the study students either did not know whether or not their school valued students' cultural identities or indicated that their school did not place importance on these. Over $65 \%$ of students did not know or indicated their teacher did not place importance on students' cultures (Section 8.2.1). However, many study participants (students and teachers) had difficulty describing their own cultural identity. Furthermore, almost all students showed confusion regarding how their cultures had been or could be linked with their mathematics learning. These results indicate that increased school, teacher, and student knowledge of their own personal cultural identities, of ngā ao Māori (Māori worlds), and Pacific Nations is needed for the goals and actions of Ka Hikitia (Ministry of Education, 2008c), the Pasifika Education Plan (Ministry of Education, 2006c), and calls from the literature regarding teacher practice (e.g., Fletcher et al., 2009; Gay, 2000; also see Section 2.3.3) and teacher education (e.g., McDonald, 2005; Mills, 2008, Villegas \& Lucas, 2002) to be fully realised. Hynds' (2007) findings indicate that such knowledge development must be carefully and sensitively managed with awareness by all of potential concomitant discomfort and conflict.

Student data indicated that parental expectations were an important motivator for many learning mathematics (Chapter Seven). However, few school-home links were noticed during lesson observations or commented on by study participants. Those in 
place (e.g., letters home regarding mathematics success, knowledge of students' family members) appeared to have positive effects on teacher-student relationships and achievement, according to participant data. The findings overall indicate that consistent with the Ministry of Education's (2006c, 2008c) goals, much more needs to be done by schools and teachers to harness the potential benefits of parental expectations. Exploration of avenues for increasing family and community input into, and responsibility for, students' mathematics learning is urgently needed to inform such practice.

This study highlights that practice recommended for teachers to enhance achievement of groups of students traditionally underserved by mathematics education (e.g., Bishop et al., 2003) can also be appreciated by, and enhance the learning of, other groups. Furthermore, no evidence emerged in this study to suggest that practices consistent with Bishop et al.'s (2003) Te Kotahitanga project are detrimental to the learning of groups other than Māori. Given the problems of resistance to change found within projects targeting specific ethnic groups (e.g., Hynds, 2007; McKenzie \& Scheurich, 2008; Theoharis, 2007), the generic nature of excellent teaching practice suggested by this research is a very valuable finding. The importance of having generic practice which supports the learning of students of many ethnic backgrounds is further highlighted by the increasingly diverse combinations of ethnic backgrounds held by many of our students (Chapter Four).

For teachers, teacher educators, school management, and policy makers, there is much to be gained from understanding and promoting a set of practices and behaviours which are suitable for enhancing achievement across ethnicities rather than attempting to implement varied strategies for different groups of students. In practice the accuracy of teachers' informal judgements about students' ethnicity are likely to vary and the time available for determining individual needs and desires limited. Determining the level of engagement of students with their cultural heritage holds greater challenges still, particularly given the apparent confusion of many students regarding the relevance of their cultural background to their everyday lives and to their learning (Chapter Eight). Therefore, attempting to draw on differing strategies for different groups may lead to judgemental and tokenistic teacher behaviours.

New Zealand schools, like many schools internationally, are becoming increasingly culturally diverse and increasingly aware of the need to address cultural issues. However, in most cases change in practice is slow, minimal, and consistent with 
Banks' (2004) 'contributions' and 'additive' approaches (Loorparg et al., 2006, Section 1.2). Low levels of community involvement in everyday school practice are common, particularly in the New Zealand secondary school sector. This study provides evidence that some students feel mathematical lessons are less conducive than lessons in other subject areas to displaying their personal identity because of the content taught. All of these factors contribute to difficulties for teachers to implement culturally responsive practice. Greater challenges still exist for mathematics teachers, as frequently held perceptions of their curriculum area include that it is unrelated to everyday life, skills-based, and answers are either right or wrong. Many textbooks and worksheets do little to dispel this image.

The results of this study, in terms of teachers' understandings of the relevance of culture to learning and the diversity of student views regarding their personal and cultural identity, are consistent with Gorinski et al.'s (2008) findings. It is hard to see how teaching can be truly culturally responsive while a mismatch between teachers' and students' ethnicities remains, teachers' cultural knowledge base is limited, and schools', teachers', students', and communities' understanding of links between culture and learning tenuous. This study, alongside others (e.g., Bishop et al., 2003; Gorinski et al., 2008) suggests that the challenges for many schools, teachers, students, and their communities to fully reflect, value, and incorporate Māori and Pasifika cultures are so great that substantial change, commitment, and effort are required for Ministry of Education policies relating to Māori and Pasifika students to be fully realised.

Internationally, there is a sound research base (e.g., Gutstein, 2003; Howard, Perry, \& Butcher, 2006; Moses \& Cobb, 2001; Nicol et al., 2007; Perry \& Howard, 2008; Philpott, Nesbit, Cahill, \& Jeffery, 2004) that could inform a New Zealand oriented development aimed at enhancing community involvement and incorporation of cultural heritage into mathematics learning.

\subsection{Topics for further exploration}

Many ideas for further research into student perceptions and teacher behaviours have emerged through this study. Further understanding of teacher care could be gained by exploring:

- what students perceive as teacher respect and the effects of mutual respect on student motivation and achievement (Section 9.1.3);

- apparent inconsistencies between students' statements regarding positive teacher behaviours (e.g., students stated that treating all students in the same 
way and treating them differently according to their culture both showed teachers' care for students (Section 8.2.1));

- areas indicated by this study to hold a mismatch between teacher behaviours and students' preferences (e.g., feedback about learning (Sections 6.2 and 7.2.2));

- the role of classroom humour in student engagement (Section 9.1.4);

- reciprocity between teacher and student behaviours (Section 9.2);

- students' and teachers' perceptions of cultural identity and how these can be developed (Chapters Six and Eight);

- how mathematics and other classrooms and schools in general can reflect more effectively students' families, cultures, cultural identities, and lives (Chapters Five and Eight);

- gender differences in how students perceive teacher care in mathematics classrooms;

- how teachers can reflect more effectively, or describe more clearly how they address, the Treaty of Waitangi in their teaching (Chapter Six);

- the use of elements of the whare model for developing teachers' practice; and

- transferability of elements of the whare model to contexts outside mathematics and outside New Zealand.

For each of the ideas for further study, the value of seeking a wide range of perspectives, in conjunction with observational data, is indicated by this study. This research was limited to the perspectives of teachers, students, and the sole researcher. A research team including members of the cultural groups involved in the study would be needed for investigations exploring cultural issues. Stakeholders important to include in such studies are cultural experts, school leadership, and parents. 


\subsection{Final comments}

Realising equity of access to mathematical achievement is urgent. Recent literature and research into mathematical learning, culturally responsive practice, and professional development programmes, alongside the current New Zealand curriculum (Ministry of Education, 2007a) and Ministry of Education policy (e.g., Ministry of Education, 2008c) position us well to make positive and lasting change towards achieving greater equity in mathematical learning. This study builds on previous research into effective teacher practice for Māori learners (Bishop et al., 2003) by indicating that strategies consistent with such practice are equally effective and important for many students of other ethnicities.

This study indicates that some teaching strategies in particular provide opportunities for students to be themselves in the mathematics class and assume greater levels of ownership of their learning than offered by traditional style lessons. Mathematical games, practical activities, investigations, and rich mathematical tasks (Ahmed, 1987) are examples of teaching strategies which offer opportunities for student decision making, fun, discussion, and humour. Moreover they can facilitate the teacher getting to know the students as people and as learners, both knowledges being essential for developing rapport and providing effective feedback on learning.

The results of this study support Macfarlane et al.'s (2008) claims that Māori worldviews have much to offer in the understanding and implementation of the key competencies (Ministry of Education, 2007a). Observational and interview data in particular indicated that many students and their teachers appreciate that a deeper level of relationship than that offered by the traditional roles of teacher and student is essential to maximise learning. Shared ownership of learning practices, flexibility to move between teacher/learner roles, caring for each other as family, and placing priority on relationships are examples that can be seen to be consistent with a Māori worldview supporting a community of practice (Lave \& Wenger, 1991) model of mathematics education. Understanding and use of examples such as these are likely to help pave the way for successful implementation of culturally responsive elements of Ministry of Education policy.

There is much still to be understood about how classrooms, schools, and mathematics teaching and learning can comfortably reflect the cultures of the school and wider community. This is an important goal yet to be realised. Caring, trusting relationships between participants in education (parents, students, teachers, policy makers, teacher educators...) that are focused on enhancing learning, alongside a 
willingness to learn about oneself, one another, and one another's cultures, offer perhaps the only sound pathway towards this aim. Understanding classroom practices that nurture teacher-student relationships across marginalised and other student groups in mathematics classrooms are one part of the puzzle. Care for one another, one another's learning, and for one another as culturally located people are essential for maximising the effectiveness of learning-focused relationships and creating culturally responsive schools.

Finally, returning to the student comment that opened Chapter One:

If they don't care, I won't.

This one short sentence by itself eloquently shows how vital the teacher's attitude is for this student's mathematics motivation and achievement. The research reported in this thesis provides ways that we as teachers can care for our students and equally importantly, ways that we can show our students that we care. Teacher care encompasses care for them as individuals, as learners, and for their cultural identities. Care is necessary for nurturing effective and happy learning environments, developing interpersonal skills and mutual understanding, and maximising many students' mathematics learning. Our aims must be to do everything possible to care for our students and to show them we care. In so doing we can aspire to our mathematics students holding tightly to the attitude:

If they care, so will I! 


\section{References}

Ahmed, A. (1987). Better mathematics: A curriculum study based on the low attainers in mathematics project. London: H.M.S.O.

Aldridge, J. M., \& Fraser, B. J. (2000). A cross-cultural study of classroom learning environments in Australia and Taiwan. Learning Environments Research, 3, 101-134.

Alexander, P. (2008). The nature of expertise: Implications for teaching and learning. Invited lecture, Victoria University of Wellington, 20 August.

Alton-Lee, A. (2003). Quality teaching for diverse students in schooling: Best evidence synthesis. Wellington: Ministry of Education.

Anae, M., Coxon, E., Mara, D., Wendt-Samu, T., \& Finau, C. (2001). Pasifika education research guidelines. Wellington: Ministry of Education.

Andersen, J. (1995). Courageous teaching: Creating a caring community in the classroom. Thousand Oaks, CA: Corwin Press.

Angrosino, M. V., \& Mays de Perez, K. A. (2000). Rethinking observation: From method to context. In N. K. Denzin \& Y. S. Lincoln (Eds.), Handbook of qualitative research ( $2^{\text {nd }}$ ed., pp. 673-702). Thousand Oaks, CA: Sage Publications.

Anthony, G., \& Walshaw, M. (2007). Effective pedagogy in mathematics/pāngarau: Best evidence synthesis. Wellington: Ministry of Education.

Antonnen, R. G. (1969). A longitudinal study in mathematics attitude. The Journal of Educational Research, 62, 467-471.

Arnaiz, P., Berruezo, P., de Haro, R., \& Martinez, R. (2005, August). Ethnic minorities in Spain: Teacher, parent, and student beliefs and educational performance in multi-cultural schools. Paper presented at the Inclusive and Supportive Education Congress International Special Education Conference (ISEC), Glasgow, Scotland.

Averill, R. (2001). Just by aiming for the middle: $A$ study of year 9 mathematics schemes. Unpublished MSc thesis, Wellington: Victoria University of Wellington.

Averill, R., \& Clark, M. (2007, April). Development of an observation tool for multicultural classrooms. Paper presented at the American Educational Research Association (AERA) Conference, Chicago.

Averill, R., Anderson, D, Easton, H., \& Hynds, A. (2004). Understandings of the implications of the Treaty of Waitangi in mathematics programs. In I. Putt, R. Faragher \& M. McLean (Eds.), Mathematics education for the third millennium: Towards 2010: Proceedings of the 27th Annual Conference of the Mathematics Education Research Group of Australasia, Townsville (pp. 5562). Sydney: MERGA.

Averill, R., Anderson, D., Easton, H., Te Maro, P., Smith, D., \& Hynds, A. (2009). Culturally responsive teaching: Three models from linked studies. Journal for Research in Mathematics Education, 40(2), 157-186.

Averill, R., Phillips, K., \& French, S. (2003). Mathematics Pacific experiences: Activities drawn from contexts in the Pacific. Masterton: Wairarapa Education Resource Centre.

Averill, R., Te Maro, P., Taiwhati, M., \& Anderson, D. (2009). Culturally responsive mathematics teaching: A bicultural model. In R. Averill \& R. Harvey (Eds.), 
Teaching secondary school mathematics and statistics: Evidence based practice: Volume Two (pp. 27-46). Wellington: NZCER.

Babad, E. (1996). How high is "high inference"? Within classroom differences in students' perceptions of classroom interaction. Journal of Classroom Interaction, 31, 1-9.

Banks, J. A. (1994). An introduction to multicultural education. Boston, MA: Allyn \& Bacon.

Banks, J. A. (2004). Approaches to multicultural curricular reform. In J. A. Banks \& C. A. M. Banks (Eds.), Multicultural education: Issues and perspectives ( $5^{\text {th }}$ ed., pp. 242-264). New York: John Wiley \& Sons.

Banks, J. A. (2006). Cultural diversity and education: Foundations, curriculum, and teaching $\left(5^{\text {th }}\right.$ ed.). Boston: Pearson Education.

Barton, B. (1995). The politics of mathematics education. In J. Neyland (Ed.), Mathematics education: Volume 2 (pp. 165-174). Wellington: Wellington College of Education.

Bills, E. J. (2000). Politeness in teacher-student dialogue in mathematics: A sociolinguistic analysis. For the Learning of Mathematics, 20(2), 40-47.

Bishop, R. (2005). Freeing ourselves from neocolonial domination in research: A kaupapa Māori approach to creating knowledge. In N. K. Denzin \& Y. S. Lincoln, (Eds), The Sage handbook of qualitative research (3 ${ }^{\text {rd }}$ ed., pp. 109-138). Thousand Oaks, CA: Sage Publications.

Bishop, R. (2008). A culturally responsive pedagogy of relations. In C. McGee \& D. Fraser (Eds.), The professional practice of teaching ( $3^{\text {rd }}$ ed., pp. 154-171). South Melbourne: Cengage Learning Australia.

Bishop, R., \& Berryman, M. (2006). Culture speaks: Cultural relationships and classroom learning. Wellington: Huia Publishers.

Bishop, R., Berryman, M., Cavanagh, T., \& Teddy, L. (2007). Te Kotahitanga phase three whanaungatanga: Establishing a culturally responsive pedagogy of relations in mainstream secondary school classrooms. Wellington: Ministry of Education.

Bishop, R., Berryman, M., Tiakiwai, S., \& Richardson, C. (2003). Te Kotahitanga: The experiences of year 9 and 10 Māori students in mainstream classrooms. Hamilton: Māori Education Research Institute (MERI), School of Education, University of Waikato.

Bishop, R., \& Glynn, T. (1999). Culture counts: Changing power relations in education. Palmerston North: Dunmore Press.

Blum, L. (1999). Ethnicity, identity, and community. In M. S. Katz, N. Noddings \& K. A. Strike (Eds.), Justice and caring: The search for common ground in education (pp. 127-145). New York: Teachers College Press.

Boaler, J. (2004, July). Promoting equity in mathematics classrooms: Important teaching practices and their effect on student learning. Paper presented at the International Conference for Mathematics Education, Copenhagen.

Boaler, J., \& Greeno, J. G. (2000). Identity, agency and knowing in mathematics worlds. In J. Boaler, (Ed.), Multiple perspectives on mathematics teaching and learning (pp. 171-200). Westport, CT: Ablex Publishing.

Borton, W.M., Preston, J., \& Bippert, J. (1996). Validating the Comer model: The effects of student, teacher and parent affective variables on reading and 
mathematics performance outcomes. Paper presented at the annual meeting of the American Educational Research Association, New York.

Bosworth, K. (1995). Caring for others and being cared for. Phi Delta Kappan, 76(9), 686-693.

Bouchey, H. A., \& Harter, S. (2005). Reflected appraisals, academic perceptions, and math/science performance during early adolescence. Journal of Educational Psychology, 97(4), 673-686.

Boulton, D., \& Hammersley, M. (2006). Analysis of unstructured data. In R. Sapsford \& V. Jupp (Eds.), Data collection and analysis ( $2^{\text {nd }}$ ed., pp. 243-259). London: Sage Publications.

Boustead, T., \& Strathdee, R. (2008). The gender-ethnic divide: Mathematics and English progress of Pākehā, Māori, and Pasifika students. New Zealand Journal of Education Studies 43(1), 33-50.

Brophy, J. E., \& Good, T. L. (1974). Teacher-student relationships: Causes and consequences. New York: Holt, Rinehart \& Winston.

Cahill, F. (2006). Crossing the road from home to secondary school: A conversation with Samoan parents. Waikato Journal of Education, 12, 57-72.

Campbell, D. T., \& Fiske, D. (1959). Convergent and discriminant validation by the multitrait-multimethod matrix. Psychological Bulletin, 56, 81-105.

Chapple, S., Jeffries, R., \& Walker, R. (1997). Māori participation and performance in education: A literature review and research programme. Report for the Ministry of Education. Wellington: New Zealand Institute of Economic Research, May.

Charmaz, K. (2006). Constructing grounded theory: A practical guide through qualitative analysis. London: Sage Publications.

Clark, M. (1995a). Raising achievement in mathematics for Pacific Island students in New Zealand. In R. P. Hunting, G. E. Fitzsimons, P. C. Clarkson \& A. J. Bishop (Eds.), Regional collaboration in mathematics education (pp. 201210). Melbourne, Australia: ICMI.

Clark, M. (1995b, April). Raising the achievement of Pacific Island students in university mathematics courses. Proceedings of the International Commission on Mathematical Education Regional Conference, Australia. Melbourne, Australia: Monash University Press.

Clark, M. (1997). Comfort in the mathematics classroom. The New Zealand Mathematics Magazine, 34(2), 14-21.

Clark, M. (1999). Māori and Pacific Islands student performance in mathematics. In Exploring issues in mathematics education (pp. 31-38). Wellington: Research and Curriculum Division, Ministry of Education.

Clark, M. (2001). Cross-cultural issues with students from the South Pacific. Australian Mathematics Teacher, 57(1), 17-20.

Connolly, J. A., Hatchette, V., \& McMaster, L. E. (1998). School achievement of Canadian boys and girls in early adolescence: Links with personal attitudes and parental and teacher support for school. Retrieved October 14, 2005, from http://www11.sdc.gc.ca/en/cs/sp/sdc/pkrf/publications/research/1998002344/page03.shtml

Cowie, B., \& Bell, B. (1999). A model of formative assessment in science education. Assessment in Education 6(1), 101-116. 
Coxon, E., Anae, M., Mara, D., Samu, T., \& Finau, C. (2001). Pacific education literature review. Wellington: Ministry of Education.

CREDE. (2004). The five standards for effective pedagogy. Center for Research on Education, Diversity and Excellence, University of California, Berkeley. Retrieved November 2005, from http://crede.berkeley.edu/standards/standards.html

Creswell, J. (1998). Qualitative inquiry and research design: Choosing among five traditions. Thousand Oaks, CA: Sage Publications.

Creswell, J. W. (2003). Research design: Qualitative, quantitative, and mixed methods approaches $\left(2^{\text {nd }}\right.$ ed.). Thousand Oaks, CA: Sage Publications.

Creswell, J. W., \& Plano Clark, V. L. (2007). Designing and conducting mixed methods research. Thousand Oaks, CA: Sage Publications.

Crooks, T., \& Flockton, L. (2002). Mathematics assessment results 2001. Dunedin: The Educational Assessment Research Unit, University of Otago.

Cunningham, C. W., \& Durie, M. H. (1998). A taxonomy, and a framework for outcomes and strategic research goals, for Māori research and development. Palmerston North: Massey University School of Māori Studies.

Cutmore, B., \& Howard, P. (1995). Identifying issues related to collaborative research in mathematics education with aboriginal communities. In R. P. Hunting, G. E. Fitzsimons, P. C. Clarkson \& A. J. Bishop (Eds.), Regional collaboration in mathematics education (pp. 221-230). Melbourne, Australia: ICMI.

Darling-Hammond, L., \& Bransford, J. (Eds.). (2005). Preparing teachers for a changing world: What teachers should learn and be able to do. San Francisco: Jossey-Bass.

Denscombe, M. (2001). The good research guide. Buckingham: Oxford University Press.

Denzin, N. K. (1985). Triangulation. In T. Husen \& T. N. Postlethwaite (Eds.), International encyclopedia of educational research 9 (pp. 5293-5295). Oxford: Pergamon.

Denzin, N. K., \& Lincoln, Y. S. (2000). Introduction: The discipline and practice of qualitative research. In N. K. Denzin \& Y. S. Lincoln (Eds.), Handbook of qualitative research $\left(2^{\text {nd }}\right.$ ed., pp. 1-28). Thousand Oaks, CA: Sage Publications.

Durie, M. (1998). Whaiora: Māori health development ( $2^{\text {nd }}$ ed.). Auckland: Oxford University Press.

Eccles, J. S. (2004). Schools, academic motivation, and stage-environment fit: In R. M. Lerner \& L. Steinberg (Eds.), Handbook of adolescent psychology $\left(2^{\text {nd }}\right.$ ed., pp. 125-154). Hoboken, NJ: John Wiley \& Sons.

Eccles, J. S., \& Midgley, C. M. (1989). Stage/environment fit: Developmentally appropriate classrooms for early adolescents. In R. E. Ames \& C. Ames (Eds.), Research on motivation in education: Goals and cognitions (Vol. 3, pp. 139-186). New York: Academic Press.

Eccles, J. S., \& Wigfield, A. (2002). Motivational beliefs, values, and goals. Annual Review of Psychology, 52, 109-132.

Education Counts. (2007). School roll summary report: School types as at 1 July 2007. Retrieved December 17, 2007, from

http://www.educationcounts.govt.nz/statistics/schooling/july school roll return s/school type/school type as at 1 july 2007 
Education Review Office. (2002). Māori students: Schools making a difference. Wellington: Education Review Office.

Education Review Office. (2008). Framework for reviews. Retrieved September 11, 2008, from http://ero.govt.nz/ero/publishing.nst/Content/ ERORevFrameworkSchools\#7.\%20Compliance\%20Matters

Elias, M. J., Zins, J. E., Weissberg, R. P., Frey, K. S., Greenberg, M. T., Haynes et al. (1997). Promoting social and emotional learning: Guidelines for educators. Alexandria, VA: Association of Supervision and Curriculum Development.

Ensign, J. (2003). Nurturing mathematics learning in the classroom. In N. Haynes, M. Ben-Avie, \& J. Ensign (Eds.), How social and emotional development add up: Getting results in maths and science education (pp. 103-119). New York: Teachers College Press.

Fennema, E., \& Sherman, J. (1978). Sex-related differences in mathematics achievement and related factors: A further study. Journal for Research in Mathematics Education, 9(3), 189-203.

Ferguson, P. B., Gorinski, R. T., Samu, T. W., \& Mara, D. (2008). Literature review on the experiences of Pasifika learners in the classroom. Wellington: Ministry of Education.

Ferguson, R. F. (2002). What doesn't meet the eye: Understanding and addressing racial disparities in high-achieving suburban schools. Retrieved June 30, 2008, from http://www.ncrel.org/gap/ferg/

Fergusson, D. M., Lloyd, M., \& Horwood, L. J. (1991). Family ethnicity, social background and scholastic achievement: An eleven year longitudinal study. New Zealand Journal of Educational Studies, 26(1), 49-63.

Fisher, D., \& Rickards, T. (1996). Associations between teacher-student interpersonal behaviour and student attitudes in mathematics classes. Paper presented at WAIER Forum 1996. Retrieved March 16, 2005, from http://education.curtin.edu.au/waier/forums/1996/fisher.html

Fisher, D. L., Waldrip, B. G., \& Chuarch, D. (2003, March). Identifying better primary teachers using student-teacher interactions. Paper presented at the Annual Meeting of the National Association for Research in Science Teaching, Philadelphia.

Fletcher, J., Parkhill, F., Fa'afoi, A., Taleni, L. T., \& O’Regan, B. (2009). Pasifika students: Teachers and parents voice their perceptions of what provides supports and barriers to Pasifika students' achievement in literacy and learning. Teaching and Teacher Education, 25, 24-33.

Flockton, L., \& Crooks, T. (1998). Mathematics assessment results 1997. Dunedin: Education Assessment Research Unit.

Flockton, L., Crooks, T., Smith, J., \& Smith, L. F. (2006). Mathematics assessment results 2005. Dunedin: Education Assessment Research Unit.

Fontana, A., \& Frey, J. H. (2000). The interview: From structured questions to negotiated text. In N. K. Denzin \& Y. S. Lincoln (Eds.), Handbook of qualitative research ( $2^{\text {nd }}$ ed., pp. 645-672). Thousand Oaks, CA: Sage Publications.

Forbes, S., Blithe, T., Clark, M., \& Robinson, E. (1990). Summary of a study of participation, performance, gender and ethnic differences in mathematics in New Zealand secondary schools and first year university courses. Wellington: Victoria University of Wellington. 
Fraser, D. (2008). Developing classroom culture: Creating a climate for learning. In C. McGee \& D. Fraser (Eds.), The professional practice of teaching $\left(3^{\text {rd }} \mathrm{ed}\right.$., pp. 1-16). South Melbourne: Cengage Learning Australia.

Friedel, J., Marachi, R., \& Midgley, C. (2002). "Stop embarrassing me!": Relations among student perceptions of teachers, classroom goals, and maladaptive behaviours. Paper presented at the annual meeting of the American Educational Research Association, New Orleans.

Gay, G. (2000). Culturally responsive teaching: Theory, research, \& practice. New York: Teachers College Press.

Good, T. L., \& Brophy, J. (2003). Looking in classrooms ( $9^{\text {th }}$ ed.). New York: Pearson Education.

Gorinski, R., Ferguson, P. B., Wendt-Samu, T., \& Mara, D. (2008). An exploratory study of eight schools: A report for the Ministry of Education. Wellington: New Zealand Council for Educational Research. Retrieved January 29, 2009, from http://www.tki.org.nz/e/community/pasifika/pdf/pasifika-learners.pdf

Gorinski, R., \& Shortland-Nuku, C. (2006, October). Building innovative communities of professional learning: A leadership practice challenge. A paper presented to the Commonwealth Council on Educational Administration and Management (CCEAM) conference, Lefkosia, Cyprus.

Grbich, C. (2007). Qualitative data analysis: An introduction. London: Sage Publications.

Greenbaum, P. E. \& Greenbaum, S. D. (1983). Cultural differences, nonverbal regulation, and classroom interaction: Sociolinguistic interference in American Indian education. Peabody Journal of Education, 61(1), 16-33.

Gutstein, E. (2003). Teaching and learning mathematics for social justice in an urban Latino school. Journal for Reseach in Mathematics Education, 34(1), 37-73.

Hackenberg, A. (2005). A model of mathematics classroom and caring relations. For the Learning of Mathematics, 25(1), 45-51.

Hattie, J. A. C. (2003, October). Teachers make a difference: What is the research evidence? Keynote presentation at the Building Teacher Quality: The ACER Annual Conference, Melbourne, Australia.

Hattie, J. A. C. (2009). Visible learning: A synthesis of over 800 meta-analyses relating to achievement. London: Routledge.

Hawk, K., \& Hill, J. (2000). Towards making achievement cool: Achievement in multicultural high schools. (A report to the Ministry of Education). Albany, Auckland: Educational Research and Development Centre, Massey University.

Hawkins, J. D., Catalano, R. F., \& Associates. (Eds.). (1992). Communities that care: Action for drug abuse prevention. San Francisco: Jossey-Bass.

Haynes, N. M., Ben-Avie, M., \& Ensign, J. (Eds.). (2003). How social and emotional development add up: Getting results in maths and science education. New York: Teachers College Press.

Heays, V., Copson, K., \& Mahon, R. (1994). Māori legends in maths. Masterton: Wairarapa Education Resource Centre.

Hemara, W. (2000). Māori pedagogies: A view from the literature. Wellington: New Zealand Council for Educational Research. 
Hill, J., \& Hawk, K. (2000). Making a difference in the classroom: Effective teaching practice in low decile, multicultural schools. Wellington: Ministry of Education.

Holloway, J. H. (2004). Closing the minority achievement gap in math. Educational Leadership, 61(5), 84-86.

Howard, P., Perry, B., \& Butcher, J. (2006). Community capacity research project final report. Sydney: Board of Studies NSW. Retrieved February 10, 2009 from: http://ab-ed.boardofstudies.nsw.edu.au/files/community capacity research project.pdf

Howard, P., Perry, B., Lowe, K., Ziems, S., \& McKnight, A. (2003). Mathematics in indigenous contexts: A case study. In L. Bragg, C. Campbell, G. Herbert \& J. Mousley (Eds.), Mathematics education research: Innovation, networking, opportunity: Proceedings of the 26th annual conference of the Mathematics Education Research Group of Australasia (pp. 436-443). Geelong, Melbourne: Deakin University.

Hudley, C., \& Daoud, A. M. (2007). High school students' engagement in school: Understanding the relationships to school context and student expectations. In F. Salili, \& R. Hoosain (Eds.), Culture, motivation and learning: A multicultural perspective (pp. 367-391). Charlotte, NC: Information Age Publishing.

Hynds, A. (2007). Navigating the collaborative dynamic: Teachers collaborating across difference. Unpublished $\mathrm{PhD}$ thesis, Victoria University of Wellington, New Zealand.

Jackson, A. W., \& Davis, G. A. (2000). Turning points 2000: Educating adolescents in the $21^{\text {st }}$ century. New York: Teachers College Press.

Jick, T. D. (1979). Mixing qualitative and quantitative methods: Triangulation in action. Administrative Science Quarterly, 24, 602-611.

Johnson, B., \& Turner, L. A. (2003). Data collection in mixed methods research. In A. Tashakkori \& C. Teddlie, (Eds.), Handbook of mixed methods in social and behavioral research (pp. 297-319). Thousand Oaks, CA: Sage Publications.

Johnson, R. B., \& Onwuegbuzie, A. J. (2004). Mixed methods research: A research paradigm whose time has come. Educational Researcher, 33(7), 14-26.

Ka'ai, T., Moorfield, J., Reilly, M., \& Mosely, S. (2004). Ki te whaiao: An introduction to Māori culture and society. Auckland: Pearson Education.

Kemper, E. A., Stringfield, S., \& Teddlie, C. (2003). Mixed methods sampling strategies in social science research. In A. Tashakkori \& C. Teddlie (Eds.), Handbook of mixed methods in social and behavioral research (pp. 273-296). Thousand Oaks, CA: Sage Publications.

Koloto, A. (2004). A Tongan perspective on development. In W. Drewery \& L. Bird (Eds.), Human development in Aotearoa: A journey through life ( $2^{\text {nd }}$ ed., pp. 61-65). Auckland: McGraw Hill.

Ladson-Billings, G. (1990). Culturally relevant teaching: Effective instruction for black students. The College Board Review, 155, 20-25.

Lave, J., \& Wenger, E. (1991). Situated learning: Legitimate peripheral participation. Cambridge: Cambridge University Press.

Lerman, S. (2006). Socio-cultural research in PME. In A. Guitierrez \& P. Boero (Eds.), Handbook of research on the psychology of mathematics education: Past, present and future (pp. 347-366). The Netherlands: Sense Publishers. 
Lock, K. J., \& Gibson, J. K. (2008). Explaining Māori under-achievement in standardised reading tests: The role of social and individual characteristics. Kōtuitui: New Zealand Journal of Social Sciences Online, 3, 1-13.

Loorparg, A., Tait, C., Yates, E., \& Meyer, L. (2006). Managing cultural diversity in New Zealand secondary schools: Report prepared for Education New Zealand. Retrieved July 7, 2006, from http://www.educationnz.org.nz/eeidf/ ManagingCulturalDiveristyfinal.pdf

Macfarlane, A. (1997). The Hikairo rationale: Teaching students with emotional and behavioural difficulties: A bicultural approach. Waikato Journal of Education, 3, 153-168.

Macfarlane, A. H. (2004). Kia hiwa ra! Listen to culture: Māori students' plea to educators. Wellington: New Zealand Council for Educational Research.

Macfarlane, A. H., Glynn, T., Grace, W., Penetito, W., \& Batemen, S. (2008). Indigenous epistemology in a national curriculum framework? Ethnicities, 8(1), 102-127.

Manu'atu, L. (2000). Tuli ke ma'u hono ngaahi mālie: Pedagogical possibilities for Tongan students in New Zealand secondary schooling. Unpublished PhD thesis, University of Auckland.

Mara, D. (1998). Pacific Islands school-parent-community liaison project: An independent evaluation report. Wellington: New Zealand Council for Educational Research.

Matthewson, C., \& Thaman, H. (1998). Designing the Rebbelib: Staff development in a Pacific multicultural environment. In C. Latchem \& F. Lockwood (Eds.), Staff development in open and flexible learning (pp. 115-126). London: Routledge.

Mayer, D., \& Marland, P. (1997). Teachers' knowledge of students: A significant domain of practical knowledge? Asia-Pacific Journal of Teacher Education, 25(1), 17-34.

McCabe, N. (1995). Twelve high school $11^{\text {th }}$ grade students examine their best teachers. Peabody Journal of Education, 70(2), 117-126.

McCarty, T. L., Lynch, R. H., Wallace, S., \& Benally, A. (1991). Classroom enquiry and Navajo learning styles: A call for reassessment. Anthropology \& Education Quarterly, 22(1), 42-59.

McChesney, J. (2009). I worked out a wicked way to do it: Students, teachers, and classroom talk. In R. Averill \& R. Harvey (Eds.), Teaching secondary school mathematics and statistics: Evidence based practice: Volume Two (pp. 47-60). Wellington: NZCER.

McCombs, B. L., \& Whisler, J. S. (1997). The learner-centered classroom and school: Strategies for increasing student motivation and achievement. San Francisco, CA: Jossey-Bass.

McCroskey, J. (1992). An introduction to communication in the classroom. Edina, MN: Burgess International Group.

McDonald, M. (2005). The integration of social justice in teacher education: Dimensions of prospective teachers' opportunities to learn. Journal of Teacher Education, 56(5), 418-435.

McGee, C., \& Penlington, C. (2001). Research on learning, curriculum and teachers' roles: Report 2: Teacher-student interaction. Hamilton: University of Waikato. 
Mclnerney, D. \& Mclnerney, V. (2006). Educational psychology: Constructing learning $\left(4^{\text {th }}\right.$ ed.). Frenchs Forest, NSW: Pearson Prentice Hall.

Mclntyre, R. (1994). Cooperative mathematics for level 7. Auckland: Longman Paul.

McKenzie, K. B., \& Scheurich, J. J. (2008). Teacher resistance to improvement of schools with diverse students. International Journal of Leadership in Education, 11(2), 117-133.

McMurchy-Pilkington, C. (1996). Ina te mahi he rangatira: Māori women as rangatira in marae kitchens. In He paepae korero: Research perspectives in Māori education. Wellington: New Zealand Council for Educational Research.

Mead, H. M. (2003). Tikanga Māori: Living by Māori values. Wellington: Huia Publishers.

Meece, J. L., \& Daniels, D. H. (2008). Child and adolescent development for educators ( $3^{\text {rd }}$ ed.). New York: McGraw Hill.

Merriam, S. B. (1998). Qualitative research and case study applications in education. San Francisco: Jossey-Bass.

Merriam, S. B. (2001). Qualitative research and case study applications in education: Revised and expanded from case study research in education. San Francisco: Jossey-Bass.

Mertens, D. M. (2003). Mixed methods and the politics of human research: The transformative-emancipatory perspective. In A. Tashakkori \& C. Teddlie (Eds.), Handbook of mixed methods in social and behavioral research (pp. 135-164). Thousand Oaks, CA: Sage Publications.

Mertens, D. M. (2005). Research methods in education and psychology: Integrating diversity with quantitative, qualitative, and mixed methods $\left(2^{\text {nd }}\right.$ ed.). Thousand Oaks, CA: Sage Publications.

Midgley, C., Feldhauer, H., \& Eccles, J. S. (1989). Student/teacher relations and attitudes toward mathematics before and after the transition to junior high school. Child Development, 60, 981-992.

Mills, C. (2008). Making a difference: Moving beyond the superficial treatment of diversity. Asia-Pacific Journal of Teacher Education, 36(4), 261-275.

Ministry for Culture and Heritage. (2008). Society: The people of New Zealand. Retrieved November 6, 2008, from http://www.teara.govt.nz/NewZealandlnBrief/Society/1/en

Ministry of Education. (1993a). Mathematics in the New Zealand curriculum. Wellington: Learning Media.

Ministry of Education. (1993b). The New Zealand curriculum framework. Wellington: Learning Media.

Ministry of Education. (1996). Te whāriki: Early childhood curriculum. Wellington: Learning Media.

Ministry of Education. (1998). Interim professional standards for primary school deputy/assistant principals and teachers. Retrieved October 10, 2008, from http://www.minedu.govt.nz/educationSectors/Schools/SchoolOperations/Empl oymentConditionsAndEvaluation/InterimProfessionalStandards.aspx\#

Ministry of Education. (1999). Health and physical education in the New Zealand curriculum. Wellington: Learning Media.

Ministry of Education. (2005). 2006 roll return guidelines. Retrieved November 8, 2006, from 
http://www.educationcounts.edcentre.govt.nz/ data/assets/pdf file/0006/727 8/sec-guide-2006-version-for-printing-2.pdf

Ministry of Education. (2006a). 2007 roll return guidelines. Retrieved November 8, 2007, from

http://www.educationcounts.edcentre.govt.nz/ data/assets/word doc/0005/7 268/2007-roll-return-guidelines-secondary-and-composit.doc

Ministry of Education. (2006b). Ngā haeata mātauranga: 2005 annual report on Māori education. Wellington: Ministry of Education.

Ministry of Education. (2006c). Pasifika education plan 2006-2010. Wellington: Learning Media.

Ministry of Education. (2007a). The New Zealand curriculum for English-medium teaching and learning in years 1-13. Wellington: Learning Media.

Ministry of Education. (2007b). Education statistics of New Zealand 2006. Wellington: The Data Management and Analysis Division, Ministry of Education.

Ministry of Education. (2007c). Te mana korero: Relationships for learning. Wellington: Ministry of Education.

Ministry of Education. (2007d). Ngā haeata mātauranga: 2006/07 annual report on Māori education. Wellington: Ministry of Education.

Ministry of Education. (2007e). Summary of findings from the evaluation of the 'effective literacy strategies: Pasifika focus' professional development project. Retrieved July 8, 2008, from http://www.tki.org.nz/r/literacy numeracy/pdf/ effective-literacy-strategies-pasifika-focus.pdf

Ministry of Education. (2008a). Deciles information. Retrieved October 24, 2008, from

http://www.minedu.govt.nz/educationSectors/Schools/SchoolOperations/Reso urcing/OperationalFunding/Deciles/DecilesInformation.aspx

Ministry of Education. (2008b). School enrolment zones: Your guide to how they work and where they are. Retrieved October 24, 2008, from http://www.schoolzones.co.nz/enrolmentzones/FAQ.aspx

Ministry of Education. (2008c). Ka Hikitia - Managing for success: Māori education strategy 2008-2012. Wellington: Author.

Ministry of Education. (n.d.) Te Kotahitanga. Retrieved August 18, 2008, from http://www.tki.org.nz/r/Māori mainstream/tekotah e.php

Mortimore, P., Sammons, P., Stoll, L., Lewis, D., \& Ecob, R. (1988). School matters: The junior years. Somerset, U.K.: Open Books.

Moses, R., \& Cobb, C. (2001). Radical equations: Math literacy and civil rights. Boston: Beacon Press.

Nakhid, C. (2003). "Intercultural" perceptions, academic achievement, and the identifying process of PI students in NZ schools. The Journal of Negro Education, 72(3) 279.

Nash, R. (2004). The explanation of social differences in reading attainment: An inspection of the P.I.R.L.S. New Zealand data. Waikato Journal of Education, $10,283-298$. 
New Zealand Association of Mathematics Teachers. (2001). New Zealand maths week 2001, 13-17 August: A week to highlight \& celebrate mathematics. Auckland: NZAMT \& Ministry of Education.

New Zealand Qualifications Authority. (2008). How many credits are needed? Retrieved November 10, 2008, from http://www.nzqa.govt.nz/ncea/forstudents/requirements.html

New Zealand Teachers Council. (2008). Draft registered teacher criteria. Retrieved November 11, 2008, from http://www.teacherscouncil.govt.nz/policy/draft.pdf

Newman, I., Ridenour, C. S., Newman, C., \& DeMarco, G. M. P. (2003). A typology of research purposes and its relationship to mixed methods. In A. Tashakkori \& C. Teddlie (Eds.), Handbook of mixed methods in social and behavioral research (pp. 167-188). Thousand Oaks, CA: Sage Publications.

Nicol, C., Archibald, J., Kelleher, H., Brown, L., Hutchingson, V., Nielsen, W., \& Owuro, J. (2007, April). Culturally responsive approaches to mathematics teaching and learning in aboriginal communities. Paper presented at the American Educational Research Association (AERA) Conference, Chicago.

Nieto, S. (1999). The light in their eyes: Creating multicultural learning communities. New York: Teachers College Press.

Nieto, S., \& Bode, P. (2008). Affirming diversity: The sociopolitical context of multicultural education ( $5^{\text {th }}$ ed.). Boston: Pearson/Allyn \& Bacon.

Noblit, G. W., Rogers, D. L., \& McCadden, B. M. (1995). In the meantime: The possibilities of caring. Phi Delta Kappan, 76(9), 680-685.

Noddings, N. (1992). The challenge to care in schools: An alternative approach to education. New York: Teachers College Press.

Noddings, N. (1993). Constructivism and caring. In R. B. Davis \& C. A. Maher (Eds.), Schools, mathematics, and the world of reality (pp. 35-50). Boston, MA: Allyn \& Bacon.

Noddings, N. (1995). Teaching themes of care. Phi Delta Kappan, 76(9), 675-679.

Noddings, N. (2003). Happiness and education. Cambridge: Cambridge University Press.

Nuthall, G. (1999). Learning how to learn: The evolution of students' minds through the social processes and culture of the classroom. International Journal of Educational Research, 31(3), 141-256.

Ocean, J. (2005). Who cares? Student's values and the mathematics curriculum. Curriculum Matters, 1, 130-151.

Ogbu, J. (1992). Understanding cultural diversity and learning. Educational Researcher, 21(8), 5-14.

Onwuegbuzie, A. J., \& Teddlie, C. (2003). A framework for analysing data in mixed methods research. In A. Tashakkori \& C. Teddlie (Eds.), Handbook of mixed methods in social and behavioral research (pp. 351-383). Thousand Oaks, CA: Sage Publications.

Openshaw, R. (2007). Evaluation of Te Kotahitanga - Phase 3. Wellington: New Zealand Post Primary Teachers Association.

Pang, V. O. (2005). Multicultural education: A caring-centered, reflective approach $\left(2^{\text {nd }}\right.$ ed. $)$. Boston: Allyn \& Bacon. 
Parkhill, F., Fletcher, J., \& Fa'afoi, A. (2005). What makes for success? Current literacy practices and the impact of family and community on Pasifika students' literacy learning. New Zealand Journal of Educational Studies, 40(1\&2), 61-84.

Patton, M. Q. (2002). Qualitative research and evaluative methods (3 ${ }^{\text {rd }}$ ed.). Thousand Oaks, CA: Sage Publications.

Pere, R. R. (1994). Ako: Concepts and learning in the Māori tradition. Wellington: Te Kohanga Reo National Trust Board.

Pere, R. R. (1997). Te wheke: A celebration of infinite wisdom ( $2^{\text {nd }}$ ed.). Gisborne: Ao Ako Global Learning New Zealand.

Pere, R. R. (2008, August). Ako, oral presentation at Te Poho-o-Rawiri marae, Gisborne, for $2^{\text {nd }}$ year student teachers enrolled at Te Wananga o Aotearoa.

Perry, B., \& Howard, P. (2008). Mathematics in indigenous contexts. Australian Primary Mathematics Classroom, 13(4), 4-10.

Perso, T. (2004). Teaching mathematics in the middle years: Is middle schooling necessary? Australian Mathematics Teacher, 61(1), 25-31.

Peterson, R. (1992). Life in a crowded place: Making a learning community. Portsmouth, $\mathrm{NH}$ : Heinemann.

Philpott, D., Nesbit, W., Cahill, M., \& Jeffery, G. (2004). Cultural diversity and education: Interface issues. St Johns, Canada: Memorial University of Newfoundland.

Pirie, S. (1998). Working toward a design for qualitative research. In A. R. Teppo (Ed.), Qualitative research methods in mathematics education (pp. 79-97). Virginia: National Council of Teachers of Mathematics.

Powell, A. (2000, April). Reflections of exemplary mathematics teachers by two African American students. Paper presented at the Annual Meeting of the American Educational Research Association, New Orleans. (ERIC Document Reproduction Service No. ED 441 760).

Pring, R. (2000). Philosophy of educational research ( $2^{\text {nd }}$ ed.). London: Continuum.

Ramsay, P., \& Oliver, D. (1995). Capacities and behaviours of quality classroom teachers. School Effectiveness and School Improvement, 6(4), 332-366.

Rata, E., with O'Brien, P., Murray, R., Mara, D., Gray, S., \& Rawlinson, C. (2001). Diversity. In V. Carpenter, H. Dixon, E. Rata, \& C. Rawlinson (Eds.), Theory in practice for educators (pp. 189-210). Palmerston North: Dunmore Press.

Roeser, R. W., \& Eccles, J. S. (1998). Adolescents' perceptions of middle school: Relation to longitudinal changes in academic and psychological adjustment. Journal of Research on Adolescence, 88(1), 123-158.

Roeser, R. W., Midgley, C. M., \& Urdan, T. C. (1996). Perceptions of the school psychological environment and early adolescents' psychological and behavioural functioning in school: The mediating role of goals and belonging. Journal of Educational Psychology, 88(3), 408-422.

Salili, F., \& Hoosain, R. (Eds.). (2007). Culture, motivation and learning: A multicultural perspective. Charlotte, NC: Information Age Publishing.

Samu, T. Wendt. (2006). The 'Pasifika umbrella' and quality teaching: Understanding and responding to the diverse realities within. Waikato Journal of Education, 12, 35-49. 
Sandelowski, M. (2003). Tables or tableaux? The challenges of writing and reading mixed methods studies. In A. Tashakkori \& C. Teddlie (Eds.), Handbook of mixed methods in social and behavioral research (pp. 321-350). Thousand Oaks, CA: Sage Publications.

Sanger, J. (1996). The complete observer? A field research guide to observation. London: Falmer Press.

Sharp, G., \& Jenner, L. (2006). Socio-economic status: Student educational achievement and participation. Retrieved July 9, 2008, from http://www.spectrumimages.co.nz/Secstatus.htmc

Sheets, R. (2005). Diversity pedagogy: Examining the role of culture in the teachinglearning process. Boston, MA: Pearson Education.

Sieber, J. (1992). Planning ethically responsible research. Newbury Park, CA: Sage Publications.

Smith, G. (1995). Whakaoho whānau: New formations of whānau as an innovative intervention into Māori cultural and educational crises. He Pukenga Korero, 1(1), 18-36.

Smith, L. T. (2005). On tricky ground: Researching the native in the age of uncertainty. In N. K. Denzin \& Y. S. Lincoln (Eds.), The Sage handbook of qualitative research ( $3^{\text {rd }}$ ed., pp. 85-107). Thousand Oaks, CA: Sage Publications.

Stoll, L., \& Fink, D. (1996). Changing our schools: Linking school effectiveness and school improvement. Buckingham, England: Open University Press.

Stoll, L., Fink, L., \& Earl, L. (2003). It's about learning (and it's about time). London: RoutledgeFalmer.

Swift, B. (2002). Preparing numerical data. In R. Sapsford \& V. Jupp (Eds.), Data collection and analysis ( $2^{\text {nd }}$ ed., pp.153-183). London: Sage Publications.

Tagg, A., \& Thomas, G. (2008). Performance of SNP students on the number framework. In Findings from the New Zealand secondary numeracy project 2007 (pp. 5-16). Wellington: Ministry of Education.

Tashakkori, A., \& Teddlie, C. (Eds.). (2003). Handbook of mixed methods in social and behavioral research. Thousand Oaks, CA: Sage Publications.

Te Maro, P., Higgins, J., \& Averill, R. (2008). Creating strong achievement gains for Māori students in English-medium mathematics classrooms. In Findings from the New Zealand Numeracy development projects 2007 (pp. 37-49). Wellington: Ministry of Education.

Teven, J., \& McCroskey, J. (1996, November). The relationship of perceived teacher caring with student learning and teacher evaluation. Paper presented at the Annual Meeting of the Speech Communication Association, San Diego, CA.

Theoharis, G. (2007). Social justice educational leaders and resistance: Toward a theory of social justice leadership. Educational Administration Quarterly, 43, 21-258.

Thomas, S., Smees, R., MacBeath, J., Robertson, P., \& Boyd, B. (2000). Valuing pupils' views in Scottish schools. Educational Research and Evaluation, 6(4), 281-316.

Thompson, A. (1998). Not for the color purple: Black feminist lessons for educational caring. Harvard Educational Review, 64(4), 522-554. 
Thorpe, V. (2007). We made this song: The group song writing processes of three adolescent rock bands. Unpublished Masters thesis, Victoria University of Wellington.

Trinick, T. (2006). Te Poutama Tau: A case study of two schools. In Findings from the New Zealand Numeracy Development Projects 2005 (pp. 103-113). Wellington: Ministry of Education.

Tupuola, A. (1998). Adolescence: Myth or reality for 'Samoan' women? Beyond the stage-like toward shifting boundaries and identities. Unpublished PhD thesis, Wellington, Victoria University of Wellington.

Tuuta, M., Bradnam, L., Hynds, A., Higgins, J., \& Broughton, R. (2004). Evaluation of the Te Kauhua Māori mainstream pilot project: Report to the Ministry of Education. Wellington: Ministry of Education.

Tyler, K. M., Uqdah, A. L., Dillihunt, M. L., Beatty-Hazelbaker, R., Conner, T., Gadson, N. et al. (2008). Cultural discontinuity: Toward a quantitative investigation of a major hypothesis in education. Educational Researcher, 37(5), 280-297.

Villegas, A. M., \& Lucas, T. (2002). Educating culturally responsive teachers: $A$ coherent approach. Albany, NY: State University of New York Press.

Watson, A. (2002). Instances of mathematical thinking among low attaining students in an ordinary secondary classroom. Journal of Mathematical Behavior, 20, 461-475.

Weiss, H. B., Kreider, H., Lopez, M. E., \& Chatman, C. M. (Eds.). (2005). Preparing educators to involve families: From theory to practice. Thousand Oaks, CA: Sage Publications.

Wentzel, K. R. (1997). Student motivation in middle school: The role of perceived pedagogical caring. Journal of Educational Psychology, 89(3), 411-419.

Werner, O., \& Schoepfle, G. M. (1987). Systematic fieldwork: Ethnographic analysis and data management: Volume 2. Thousand Oaks, CA: Sage Publications.

Wigfield, A., \& Harold, R. D. (1992). Teacher beliefs and children's achievement self-perceptions: A developmental perspective. In D. H. Schunk \& J. L. Meese (Eds.), Student perceptions in the classroom (pp. 95-121). Hillsdale, NJ: Earlbaum.

Wigfield, D., Eccles, J.S., Maclver, D., Reuman, D., \& Midgley, C. (1991). Transitions during early adolescence: Changes in children's domain-specific self-perceptions and general self-esteem across the transition to junior high school. Developmental Psychology, 27, 552-565.

Wlodkowski, R. J., \& Ginsberg, M. B. (1995). A framework for culturally responsive teaching. Educational Leadership, 53(1), 17-21.

Wragg, E. C. (1999). An introduction to classroom observation ( $2^{\text {nd }}$ ed.). London: Routledge. 
Appendices 
Appendix 1: Literature (main empirical studies)

\begin{tabular}{|c|c|c|c|}
\hline $\begin{array}{c}\text { Author/s and Study } \\
\text { Focus }\end{array}$ & Context & Participants & Method \\
\hline $\begin{array}{l}\text { Bishop, Berryman, } \\
\text { Tiakiwai, \& Richardson } \\
\text { (2003) } \\
\text { Experiences of Māori } \\
\text { students in English } \\
\text { medium secondary } \\
\text { schools }\end{array}$ & $\begin{array}{l}\text { New Zealand } \\
\text { English-medium } \\
\text { secondary schools } \\
\text { Mix of decile, single sex } \\
\text { and co-educational } \\
\text { schools }\end{array}$ & $\begin{array}{l}70 \text { Year } 9 \text { and } 10 \\
\text { students, } \\
50 \text { parents, } \\
80 \text { teachers, and } \\
\text { four principals across } \\
\text { four urban and rural } \\
\text { town schools }\end{array}$ & $\begin{array}{l}\text { Group and individual } \\
\text { interviews } \\
\text { Carried out in the } \\
\text { second half of the year, } \\
\text { hence classroom } \\
\text { relationships were } \\
\text { already established }\end{array}$ \\
\hline $\begin{array}{l}\text { Hawk \& Hill (2000) } \\
\text { Effective teaching in low } \\
\text { decile multicultural } \\
\text { schools }\end{array}$ & $\begin{array}{l}\text { New Zealand } \\
8 \text { low decile multicultural } \\
\text { secondary schools ( } 7 \text { in } \\
\text { Auckland and } 1 \text { in } \\
\text { Wellington) } \\
\text { All schools had high } \\
\text { proportions of Pasifika } \\
\text { students }\end{array}$ & $\begin{array}{l}89 \text { teachers, group } \\
\text { discussions with six } \\
\text { students from each of } 12 \\
\text { 'effective' teachers }\end{array}$ & $\begin{array}{l}100 \text { lesson observations, } \\
\text { teacher interviews, } \\
\text { student group } \\
\text { discussions } \\
\text { Carried out June- } \\
\text { November, hence } \\
\text { classroom relationships } \\
\text { were already established }\end{array}$ \\
\hline $\begin{array}{l}\text { Brophy \& Good (1974) } \\
\text { Teacher-student } \\
\text { relationships }\end{array}$ & U.S.A. & $\begin{array}{l}\text { Informed by a range of } \\
\text { studies into teacher- } \\
\text { student interactions }\end{array}$ & $\begin{array}{l}\text { Informed by a range of } \\
\text { studies }\end{array}$ \\
\hline $\begin{array}{l}\text { Haynes, Ben-Avie, \& } \\
\text { Ensign (2003) } \\
\text { Effect of positive } \\
\text { teacher-student } \\
\text { relationships on } \\
\text { mathematics and } \\
\text { science learning }\end{array}$ & $\begin{array}{l}\text { U.S.A. } \\
\text { Range of studies } \\
\text { reported }\end{array}$ & $\begin{array}{l}\text { Range of studies } \\
\text { reported }\end{array}$ & $\begin{array}{l}\text { Range of studies } \\
\text { reported }\end{array}$ \\
\hline $\begin{array}{l}\text { Bosworth (1995) } \\
\text { Caring teachers }\end{array}$ & $\begin{array}{l}\text { U.S.A. } \\
\text { One city and one } \\
\text { suburban school }\end{array}$ & $\begin{array}{l}\text { Over } 1006^{\text {th }}-8^{\text {th }} \text { grade } \\
\text { students (identified by } \\
\text { teachers as most caring } \\
\text { and least caring) }\end{array}$ & Interviews \\
\hline $\begin{array}{l}\text { Hackenberg (2005) } \\
\text { Mathematical learning } \\
\text { and caring relations }\end{array}$ & $\begin{array}{l}\text { U.S.A. } \\
\text { One rural middle school } \\
\text { in Georgia }\end{array}$ & Four $6^{\text {th }}$ grade students & $\begin{array}{l}\text { Video analysis } \\
\text { (constructivist teaching } \\
\text { experiment of } \\
\text { quantitative reasoning } \\
\text { with fractions) }\end{array}$ \\
\hline $\begin{array}{l}\text { Noblit, Rogers, \& } \\
\text { McCadden (1995) } \\
\text { Caring teacher- student } \\
\text { relationships }\end{array}$ & $\begin{array}{l}\text { U.S.A.. } \\
\text { One inner-city elementary } \\
\text { school, } 65 \% \text { students low } \\
\text { income African American, } \\
35 \% \text { middle-upper class } \\
\text { American European }\end{array}$ & $\begin{array}{l}\text { Two teachers (Year } 2 \\
\text { and Year 4) and their } \\
\text { classes, the two } \\
\text { teachers had very } \\
\text { different classroom } \\
\text { styles }\end{array}$ & $\begin{array}{l}\text { Classroom observations } \\
\text { one day each week, filed } \\
\text { notes and interviews } \\
\text { with teachers and } \\
\text { students }\end{array}$ \\
\hline $\begin{array}{l}\text { Bills (2000) } \\
\text { Teacher politeness }\end{array}$ & U.K. & $\begin{array}{l}\text { Two } 17 \text { year old boys } \\
\text { studying A level Pure } \\
\text { Mathematics }\end{array}$ & $\begin{array}{l}\text { Interviews within a larger } \\
\text { study observing students } \\
\text { through their year's } \\
\text { schooling }\end{array}$ \\
\hline $\begin{array}{l}\text { Friedel, Marachi, \& } \\
\text { Midgley (2002) } \\
\text { Taking care not to } \\
\text { embarrass students }\end{array}$ & $\begin{array}{l}\text { U.S.A. } \\
\text { Four economically and } \\
\text { ethnically diverse mid- } \\
\text { West school districts } \\
\text { Mathematics }\end{array}$ & $\begin{array}{l}968 \text { grade } 7 \text { students } \\
\text { (61\% European } \\
\text { American, } 29 \% \text { African } \\
\text { American, } 10 \% \text { other } \\
\text { ethnic backgrounds) }\end{array}$ & Survey \\
\hline
\end{tabular}




\section{Appendix 2: Further literature}

\begin{tabular}{|c|c|c|}
\hline $\begin{array}{l}\text { Authors (Books and } \\
\text { Reports) }\end{array}$ & Main Context & Focus \\
\hline Noddings (1992) & U.S.A. & Caring school environments and teacher care \\
\hline Noddings (1993) & U.S.A. & Constructivism and teacher care \\
\hline Noddings (2003) & U.S.A. & Teacher care \\
\hline $\begin{array}{l}\text { Elias, Zins, Weissberg, } \\
\text { Frey, Greenberg, } \\
\text { Haynes, Kessler, } \\
\text { Schwab-Stone, \& } \\
\text { Shriver (1997) }\end{array}$ & U.S.A. & $\begin{array}{l}\text { Promoting social and emotional learning, teacher care, } \\
\text { caring classroom community }\end{array}$ \\
\hline $\begin{array}{l}\text { McCombs \& Whisler } \\
\text { (1997) }\end{array}$ & U.S.A. & $\begin{array}{l}\text { Learner-centred classrooms, caring classroom } \\
\text { communities }\end{array}$ \\
\hline Peterson (1992) & U.S.A. & Classroom environment \\
\hline Gay (2000) & U.S.A. & $\begin{array}{l}\text { Culturally responsive teaching } \\
\text { Power of caring within culturally responsive teaching }\end{array}$ \\
\hline Ladson-Billings (1995) & U.S.A. & $\begin{array}{l}\text { Culturally responsive teaching framework and } \\
\text { description }\end{array}$ \\
\hline Villegas \& Lucas (2002) & U.S.A. & Culturally responsive teacher education \\
\hline $\begin{array}{l}\text { Wlodkowski \& Ginsberg } \\
(1994,1995)\end{array}$ & U.S.A. & $\begin{array}{l}\text { Culturally responsive teaching, framework and } \\
\text { description }\end{array}$ \\
\hline Good \& Brophy (2003) & U.S.A. & Research on teaching and classroom practice \\
\hline Alton-Lee (2003) & New Zealand & $\begin{array}{l}\text { Effective practice for teaching diverse students, } \\
\text { synthesis of literature on teaching diverse learners }\end{array}$ \\
\hline $\begin{array}{l}\text { Anthony \& Walshaw } \\
\text { (2007) }\end{array}$ & New Zealand & $\begin{array}{l}\text { Effective mathematics teaching practice, synthesis of } \\
\text { literature on mathematics teaching and learning }\end{array}$ \\
\hline
\end{tabular}




\section{Appendix 3: Observation schedule}

Note: Actual Size A3

Averill Observation Schedule School:

Time/Date:

\#students:

Purpose: To record behaviours of teacher and students which indicate establishing or maintaining caring teacher-student relationships and student engagement.

$(\checkmark$ indicates infrequent use, $\mathcal{X}$ indicates some use, indicates frequent use, circling indicates negative effect, $(\mathrm{S})$ indicates more subjective aspects, $\mathrm{C}=$ consequence (also in anecdotal notes))

\section{Caring for the Individual}

Physical aspects

- Uses sustained eye contact

- Smiles

affirming neutral negative Body language (S)

- Shows they like the students (S)

\section{Verbal aspects}

- Greets and Farewells students

- Uses 'we' (LI/other)

- Uses names

Lesson Overview (topic/focus, style)

Lesson content/contexts allow for sharing of themselves: $\square$ deliberate spontaneous

Not at all

Many opportunities taken

- Explains their practice

- Uses praise (general, specific and valid, specific and invalid)

- Uses humour

- Uses classroom guidelines to facilitate learning rather than for control

- Admits and apologises when appropriate

- Gives of themselves

- Speaks quietly 1 to 1 when appropriate

Aural and Empathetic aspects

- Shows empathy (S)

- Responds to needs (S)

- Non-confrontational

- Shows they listen

- Shows they know the students e.g., where they live, families, sports success...

\section{Caring for the Individual as Culturally Located Use of Contexts}

- Drawing maths contexts from students' culturally located expertise, knowledge, interests and experiences

- Apparent knowledge of and valuing of culturally relevant knowledge and practice

\section{Teacher Behaviour}

- Acknowledges students' cultural expertise and knowledge

- Understands and allows for students' code/s of behaviour, cultural responsibilities

- Uses greetings and other words in te reo Maori/PN language

\section{Description of first 5 minutes of lesson}

Passage into community of practice:

Re-establishing relationships/rapport:

Signalling maths focus: 


\section{Caring for Individuals' Mathematics Progress}

\section{Encouragement}

- Encourages to work hard

- Encourages to do well

- Acknowledges maths success/effort

- Enables affirmation by peers

- Encourages to take pride in their work

- Persistently maintains focus of students on lesson

\section{Assistance}

- Helps when needed

- Uses pre-re-teaching e.g., squares/square roots before teaching Pythagoras

- Refers to diagnostic information. C:

- Checks for understanding of content/language. C:

\section{Learning Community}

- Uses safe questioning practices

- Expands on students' responses

- Engenders shared sense of endeavour

- Engenders sense of trust (S)

- Encourages and expects respectfulness

- Involves students in decision making

- Incorporates collaborative work

- Draws maths contexts from students' interests and experiences

\section{Student-Initiated Interactions with Teacher}

- Humour

- Greeting/Farewell

- New conversation about class activity (maths/not maths)

- Seek approval for their work affirming neutral negative Body language

- Show they want to be involved (S)

- Respond to teacher (maths/not maths)

- Ask questions (maths/not maths)

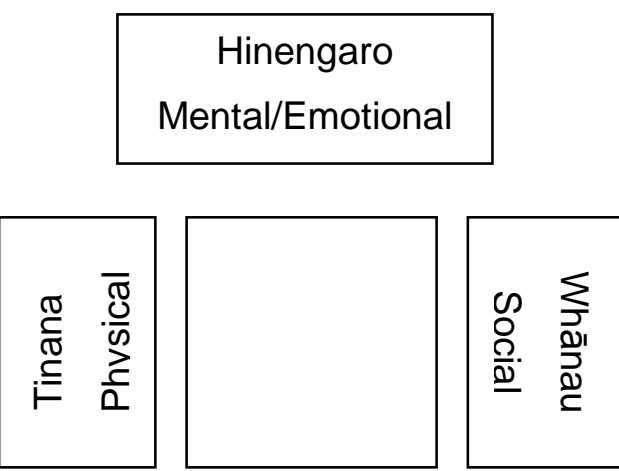

Wairua

Spiritual

Teacher interacts (maths/not maths) 1 to 1 with $\square$ Some $\square$ Half the class $\square$ All/Nearly All

Engagement (whole class one colour, individuals another colour) (S) Proportion of class highly engaged:

\begin{tabular}{lc}
\hline None & All \\
\hline None & All \\
\hline None & All \\
\hline None & All
\end{tabular}

first $1 / 4$ of the lesson

second $1 / 4$ of the lesson

third $1 / 4$ of the lesson

last $1 / 4$ of the lesson

Class engaged to indicated teacher expectations: $\square$ Yes $\square$ No (S) 


\section{Appendix 4: Initial student questionnaires}

Name:

Date of birth:

Male

Female

How true are each of the following statements for you?

Tick the box that most closely shows how you feel.

I like learning Maths

I work hard in Maths

My Maths teacher wants to help me learn

I like the way my teacher treats me when I make a mistake

My teacher encourages me to do my best

My teacher likes the class to ask questions

My teacher expects the class to work hard

My teacher treats me with respect

My teacher makes me feel they care about me

My culture is important to me

\begin{tabular}{|l|l|l|l|l|}
\hline Always & $\begin{array}{c}\text { Most of } \\
\text { the } \\
\text { time }\end{array}$ & $\begin{array}{c}\text { Some- } \\
\text { times }\end{array}$ & $\begin{array}{c}\text { Not } \\
\text { often }\end{array}$ & Never \\
\hline & & & & \\
\hline & & & & \\
\hline & & & & \\
\hline & & & & \\
\hline & & & & \\
\hline & & & & \\
\hline & & & & \\
\hline & & & & \\
\hline & & & & \\
\hline
\end{tabular}

\section{Circle the answer that most closely shows how you feel.}

My teacher knows how good I am at Maths.

Yes No Don't know

It is important to me to improve my Maths skills this year. Yes No

I am certain I can do the Maths work in this class if I try. Yes No Don't know

\section{Tick all options that apply to you.}

When I work hard in Maths it is because

I want to work well for my teacher

my parents expect me to

I want to learn the work

my friends work hard

it will be useful for my job in the future

it will be useful to me in life other than for a job

other (please explain) 
Tick all options that apply to you.

I would like my Maths teacher to know about my

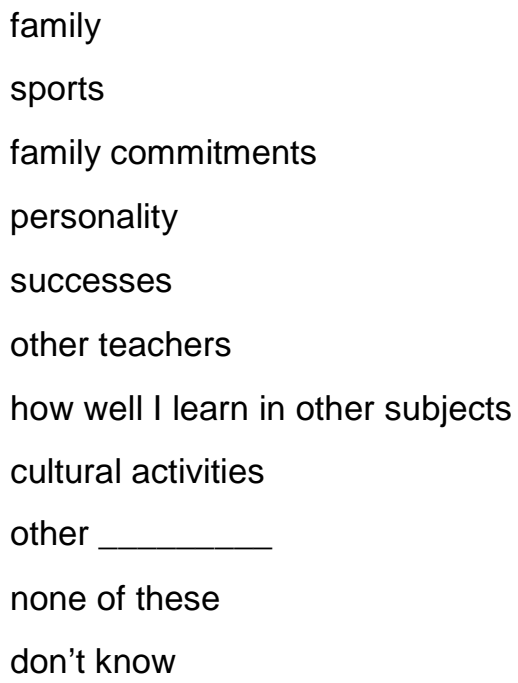

Tick all the ethnic groups you are part of.

Māori

Pacific Islands (please write which country)

NZ European

Indian

Chinese

other (please explain)

List the languages you can speak.

List the languages you speak at home. 
Tick the box that most closely shows how you feel.

I would like my teacher to know about my culture/ethnicity

I would like my teacher to know about the Māori world

I would like my teacher to know about Pacific Nations things

I would like my teacher to use Māori and Pacific Nations examples in Maths

\begin{tabular}{|l|l|l|l|l|}
\hline Always & $\begin{array}{c}\text { Most of } \\
\text { the } \\
\text { time }\end{array}$ & $\begin{array}{c}\text { Some- } \\
\text { times }\end{array}$ & $\begin{array}{c}\text { Not } \\
\text { often }\end{array}$ & Never \\
\hline & & & & \\
\hline & & & & \\
\hline & & & & \\
& & & & \\
\hline & & & & \\
\hline
\end{tabular}

What are the most important things your teacher should do to have a good relationship with your class?

Thank you very much for completing this questionnaire. 
Ingoa: Wā Whānau:

Tāne

Wahine

Pēhea te tika o ngā rārangi nei e pā ana ki a koe?

Tohua te pouaka e hāngai tata ana ki ōu ake whakaaro.

He pai ki au te whakamātau Pangarau.

He kaha taku mahi i te Pangarau.

E hiahia ana taku kaiako Pangarau ki te awhi i au i roto i aku mahi.

He pai ki au te āhuatanga whakahaere a taku kaiako i te wā hē aku mahi.

He tautoko taku kaiako kia eke aku mahi ki te taumata e taea ai e au.

He pai ki te kaiako, kia pātaitai ngā akonga.

He tūmanako na te kaiako ka mahi kaha ngā akonga.

He manaaki nui taku kaiako i au.

Ka uru mai te whakaaro nei na, he aro pai taku kaiako mōku.

He mea nui ki au ōku Tikanga.

\begin{tabular}{|l|l|l|l|l|}
\hline $\begin{array}{c}\text { i ngā } \\
\text { wā } \\
\text { katoa }\end{array}$ & $\begin{array}{c}\text { i te } \\
\text { nuinga } \\
\text { o te wā }\end{array}$ & $\begin{array}{c}\text { ètahi } \\
\text { wā }\end{array}$ & $\begin{array}{c}\text { iti nei } \\
\text { te wā }\end{array}$ & kore \\
\hline & & & & \\
\hline & & & & \\
\hline & & & & \\
\hline & & & & \\
\hline & & & & \\
\hline & & & & \\
\hline & & & & \\
\hline & & & & \\
\hline & & & & \\
\hline
\end{tabular}

\section{Porowhitatia te whakautu e hāngai tata ana ki ōu ake whakaaro.}

He mōhio taku kaiako ki tōku pai ki te mahi Pangarau.

$\mathrm{Ae}$

Kāo

Aua

He mea nui ki au ki te hiki ake i taku mōhio ki te mahi

$\mathrm{Ae}$

Kāo

Aua

Pangarau i tēnei tau.

Me taku mōhio anō, ki te ngana atu au, ka taea e au

$\mathrm{Ae}$

Kāo

Aua

ngā mahi Pangarau i roto i te akomanga. 
Tohua katoatia ngā ara whiringa e pā ana ki a koe.

Ina kaha taku mahi Pangarau, na te mea

- he hiahia kia pai aku mahi mo taku kaiako

- he wawata na aku mātua kia.....

ㄱe hiahia nōku ki te mahi

he mahi kaha na ōku hoa

- ka whai hua taku mahi a ngā wa e heke mai nei

- he hua kei roto i tēnei mōku, tēra i tua atu o te mahi

- Ėtahi atu (tēna whakamaaramatia)

Tohua katoatia ngā ara whiringa e pā ana ki a koe.

Ka hiahia au kia mōhio taku kaiako Pangarau ki:

๑ taku whānau

口 aku mahi tākaro

口 tāku e ngākau nui ana ki taku whānau

\ tōku tuakiritanga

व aku mahi angitu

口 ētahi atu o aku kaiako

\ te mātau e pēhea ana au i roto i ētahi atu o aku akoranga mahi

\ te mōhio ki aku mahi ā-Tikanga

व ētahi atu

Kāore he pānga ki ēnei

๑ Kāore au e mōhio 


\section{Tohua ngā tuakiri e pā ana ki a koe}

๑ Māori

- Te Moana nui-ā-Kiwa (Pacific) (tohua no tēhea whenua)

口 N.Z. Pākeha

๑ İnia

口 Haina

口 Ėtahi atu (whakaaturia mai)

Whakarārangitia ngā reo e kōrero ana koe.

Whakarārangitia ngā reo kei te kōrerotia e koe i tōu kāinga.

Tohua te pouaka e hāngai tata ana ki ōu ake whakaaro.

Ka hiahia au kia mōhio mai taku kaiako ki ōku ake tikanga/ā-iwi.

Ka hiahia au kia mōhio taku kaiako ki te Ao Māori.

Ka hiahia au kia mōhio taku kaiako ki ngā āhuatanga o ngā iwi o Te Moana-nui-aKiwa.

Ka hiahia au kia whakamahia e taku kaiako ngā tūmomo tauira e pā ana ki ngā mahi Pangarau a te Māori me ngā iwi o Te Moana-nui-a-Kiwa.

\begin{tabular}{|c|c|c|c|c|}
\hline $\begin{array}{c}\text { i ngāa } \\
\text { wā } \\
\text { katoa }\end{array}$ & $\begin{array}{c}\text { i te } \\
\text { nuinga } \\
\text { o te wā }\end{array}$ & $\begin{array}{c}\text { ētahi } \\
\text { wā }\end{array}$ & $\begin{array}{c}\text { iti nei } \\
\text { te wā }\end{array}$ & kore \\
\hline & & & & \\
\hline & & & & \\
\hline & & & & \\
\hline & & & & \\
& & & & \\
\hline
\end{tabular}


He aha ngā tino mahi whakaū ki te whakawhanaunga ma to kaiako i roto i to rōpū akomanga?

Ka nui te mihi mo ā kōutou whakautu i roto i tēnei pepa pātaitai. 
O le a le sa'o o nei fa'amatalaga mo oe?

Fa'ailoga le pusa e te iloa e fetaui ma lou manatu.

\begin{tabular}{|c|c|c|c|c|}
\hline $\begin{array}{c}\text { Taimi } \\
\text { uma }\end{array}$ & $\begin{array}{c}\text { I tele } \\
0 \\
\text { taimi }\end{array}$ & $\begin{array}{c}\text { I nisi } \\
\text { taimi }\end{array}$ & $\begin{array}{c}\text { Le } \\
\text { tupu } \\
\text { so'o }\end{array}$ & Le tupu \\
\hline & & & & \\
\hline & & & & \\
\hline & & & & \\
\hline & & & & \\
\hline & & & & \\
\hline & & & & \\
\hline & & & & \\
\hline & & & & \\
\hline & & & & \\
\hline
\end{tabular}

Ou te fiafia e a'oa'o i le numera

Ou te toaga e a'oa'o le numera

O lo'u faiaoga i le numera e mana'o e fesoasoani mai ia te a'u

Ou te fiafia i auala e fesoasoani mai ai lota faiaoga pe a ou sese

E fa'amalosi mai a'u e lo'u faiaoga ia ou taumafai atili

E fiafia le faiaoga pe a fesili atu le vasega ia te ia

E mana'o le faiaoga ia tauivi ma fa'amalolosi le vasega

E fa'aaloalo lo'u faiaoga ia te a'u

O lo'u faiaoga na te faailoa mai lona alofa ia te a'u

E taua la'u aganu'u ia te a'u

\section{Fa'ailoga le tali e latalata i ou lagona.}

E iloa e le faiaoga lo'u lelei i numera.

loe Leai Leiloa

E taua le fa'alelei atili o la'u numera i lenei tausaga

loe Leai Leiloa

Ou te mautinoa e mafai ona ou faia le numera pe a ou taumafai

loe Leai Leiloa 


\section{Filifili uma mea e fetaui ma oe.}

Ou te galue malosi i le numera aua

$\begin{array}{ll}\square & \text { E fiafia ai lou faiaoga } \\ \square & \text { E mana'o iai ou matua } \\ \square & \text { Ou te fia iloa atili le numera } \\ \square & \text { E galulue malolosi ai a'u uo } \\ \square & \text { E taua mo sa'u galuega i le lumana'i } \\ \square & \text { E taua mo lo'u lumana'i } \\ \square & \text { Ma nisi mea (fa'amolemole fa'amatala mai) }\end{array}$

\section{Filifili uma mea e fetaui ma oe.}

Oute fiafia pe a iloa e la'u faiaoga

口 lo'u aiga

a'u ta'aloga

口 a'u galuega i totonu i lo'u aiga

口 la'u amio

口 lo'u taunuuga

口 isi o'u faiaoga

口 la'u taumafaiga i isi mataupu

口 fa'atinoga o la'u aganu'u

Ma nisi mea

$\square \quad$ E leai se mea

$\square \quad$ Ou te leiloa

\section{Faailoa le itu aiga e te lavea a?}

Māori

口 Tagata Pasifika (fa'amolemole tusi mai le atunu'u

口 Papalagi

Initia

$\square \quad$ Saina

$\quad$ O isi (fa'amolemole tusi mai

Tusi mai Gagana e mafai ona e tautala ai

Tusi mai Gagana e te tautala ai I lo'u aiga 
Fa'ailoga le pusa e te iloa e fetaui ma lou manatu.

Oute mana'o ia iloa e lo'u faiaoga la'u aganu'u

Oute mana'o ina ia iloa e lo'u faiaoga le aganu'u fa'aMāori

Oute mana'o ina ia iloa e lo'u faiaoga le agunu'u fa'apasifika

Oute mana'o ina ia fa'aogaina e lo'u faiaoga fa'ata'ita'iga mai

le aganu'u fa'aMāori ma fa'apasifika

\begin{tabular}{|c|c|c|c|c|}
\hline $\begin{array}{c}\text { Taimi } \\
\text { uma }\end{array}$ & $\begin{array}{c}\text { I tele } \\
0 \\
\text { taimi }\end{array}$ & $\begin{array}{c}\text { I nisi } \\
\text { taimi }\end{array}$ & $\begin{array}{c}\text { Le } \\
\text { tupu } \\
\text { so'o }\end{array}$ & Le tupu \\
\hline & & & & \\
\hline & & & & \\
\hline & & & & \\
& & & & \\
\hline & & & & \\
& & & & \\
\end{tabular}

O a mea taua e tatau ona fai e le faiaoga e maua ai le mafutaga lelei ma le vasega?

Fa'afetai tele mo le taliina o nei fesili. 


\section{Appendix 5: Second student questionnaires}

Name: Date of birth:

Male

Female

How true are each of the following statements for you?

Tick the box that most closely shows how you feel.

I like learning Maths

I work hard in Maths

My Maths teacher wants to help me learn

I like the way my teacher treats me when I make a mistake

My teacher encourages me to do my best

My teacher likes the class to ask questions

My teacher expects the class to work hard

My teacher treats me with respect

My teacher makes me feel they care about me

My teacher answers my questions about the work

\begin{tabular}{|l|l|l|l|l|}
\hline Always & $\begin{array}{c}\text { Most } \\
\text { of } \\
\text { the } \\
\text { time }\end{array}$ & $\begin{array}{c}\text { Some- } \\
\text { times }\end{array}$ & $\begin{array}{c}\text { Not } \\
\text { often }\end{array}$ & Never \\
\hline & & & & \\
\hline & & & & \\
\hline & & & & \\
\hline & & & & \\
\hline & & & & \\
\hline & & & & \\
\hline & & & & \\
\hline & & & & \\
\hline & & & & \\
\hline & & & & \\
\hline
\end{tabular}

Circle the answer that most closely shows how you feel.

My teacher knows how good I am at Maths

Yes No Don't know

It is important to me to improve my Maths skills this year

Yes

No

I am certain I can do the Maths work in this class if I try

Yes No

Don't know

Tick all options that apply to you.

When I work hard in Maths it is because

I want to work well for my teacher

my parents expect me to

I want to learn the work

my friends work hard

it will be useful for my job in the future

it will be useful to me in life other than for a job

I want to be successful in NCEA next year

other (please explain) 
Tick all options that apply to you.

My maths teacher knows something about

my family

my sports

my family commitments

my personality

my successes

my other teachers

how well I can learn in maths

how well I learn in other subjects

my cultural activities at school

my cultural activities out of school

other

none of these

don't know

Tick the box that most closely shows how you feel.

My teacher knows my ethnicity/culture

My teacher knows about the Mãori world

My teacher knows about Pacific Nations things

My teacher uses Māori and Pacific Nations examples in Maths

\begin{tabular}{|c|c|c|}
\hline Yes & No & $\begin{array}{c}\text { I don't } \\
\text { know }\end{array}$ \\
\hline & & \\
\hline & & \\
\hline & & \\
\hline & & \\
\hline
\end{tabular}

What are the most important things your teacher does to have a good relationship with your class? 
These ideas came from students in Term 1.

Tick the box that most closely shows how you feel.

I like it when my maths teacher smiles

I like it when my maths teacher uses humour

I like it when my maths teacher rewards good work

My maths teacher knows my name

I like it when we have fun learning maths

I like it when we try doing harder maths work

My maths teacher likes teaching our class

I like it when my maths teacher gives us choices

I like it when my maths teacher is patient

My maths teacher listens to me

\begin{tabular}{|l|l|l|l|l|}
\hline $\begin{array}{c}\text { Strongly } \\
\text { Agree }\end{array}$ & Agree & Neutral & Disagree & $\begin{array}{c}\text { Strongly } \\
\text { Disagree }\end{array}$ \\
\hline & & & & \\
\hline & & & & \\
\hline & & & & \\
\hline & & & & \\
\hline & & & & \\
\hline & & & & \\
\hline & & & & \\
\hline & & & & \\
\hline & & & & \\
\hline & & & & \\
\hline
\end{tabular}

How do you like to get your teacher's feedback about your maths progress?

Do you think it is important that your teacher cares about your maths learning? Give reasons for your answer.

Thank you very much for completing this questionnaire. 
Ingoa:

Pēhea te tika o ngā rārangi nei e pā ana ki a koe?

Tohua te pouaka e hāngai tata ana ki ōu ake whakaaro.

He pai ki au te whakamātau Pangarau. He kaha taku mahi i te Pangarau.

E hiahia ana taku kaiako Pangarau ki te awhi i au i roto i aku mahi.

He pai ki au te āhuatanga whakahaere a taku kaiako i te wā hē aku mahi.

He tautoko taku kaiako kia eke aku mahi ki te taumata e taea ai e au.

He pai ki te kaiako, kia pātaitai ngā akonga.

He tūmanako na te kaiako ka mahi kaha ngā akonga.

He manaaki nui taku kaiako i au.

Ka uru mai te whakaaro nei na, he aro pai taku kaiako mōku.

Ngā whakautu a taku kaiako ki aku pātai mo te mahi.

\begin{tabular}{|c|c|c|c|c|}
\hline $\begin{array}{c}\text { i ngāa } \\
\text { wā } \\
\text { katoa }\end{array}$ & $\begin{array}{c}\text { i te } \\
\text { nuinga } \\
\text { o te wā }\end{array}$ & $\begin{array}{c}\text { ètahi } \\
\text { wā }\end{array}$ & $\begin{array}{c}\text { iti nei } \\
\text { te wā }\end{array}$ & kore \\
\hline & & & & \\
\hline & & & & \\
\hline & & & & \\
\hline & & & & \\
\hline & & & & \\
\hline & & & & \\
\hline & & & & \\
\hline & & & & \\
\hline & & & & \\
\hline
\end{tabular}

\section{Porowhitatia te whakautu e hāngai tata ana ki ōu ake whakaaro.}

He mōhio taku kaiako ki tōku pai ki te mahi Pangarau. Ae Kāo Aua He mea nui ki au ki te hiki ake i taku mōhio ki te mahi Ae Kāo Aua Pangarau i tēnei tau.

Me taku mōhio anō, ki te ngana atu au, ka taea e au Ae Kāo Aua ngā mahi Pangarau i roto i te akomanga. 
Tohua katoatia ngā ara whiringa e pā ana ki a koe.

Ina kaha taku mahi Pangarau, na te mea

- he hiahia kia pai aku mahi mo taku kaiako

- he wawata na aku mātua kia.....

口 he hiahia nōku ki te mahi

- he mahi kaha na ōku hoa

ka whai hua taku mahi a ngā wa e heke mai nei

- he hua kei roto i tēnei mōku, tēra i tua atu o te mahi

- kei te hiahia ahau kia whai hua au i te NCEA a te tau e tū mai nei

口 Ėtahi atu (tēna whakamaaramatia)

Tohua katoatia ngā ara whiringa e pā ana ki a koe.

He mōhio taku kaiako pangarau ki ētahi mea e pā ana ki:

口 taku whānau

- aku mahi tākaro

口 tāku e ngākau nui ana ki taku whānau

口 tōku tuakiritanga

- aku mahi angitu

- taku pai ki te mahi pangarau

- taku pai ki te ako i ètahi atu marau

- aku mahi taha tikanga i te kura

口 aku mahi taha tikanga i waho o te kura

口 ètahi atu

【 Kāore he pānga ki ēnei

๑ Kāore au e mōhio

Tohua te pouaka e hāngai tata ana ki ōu ake whakaaro.

he mōhio taku kaiako ki taku iwi/taha tikanga he mōhio taku kaiako ki te Ao Māori

he mōhio taku kaiako ki ngā pānga a ngā iwi o Te Moana Nui ā Kiwa

whakamahia ai e taku kaiako ètahi tauira pangarau a te Māori, me a ngā iwi

te Moana Nui ā Kiwa

\begin{tabular}{|l|l|l|}
\hline $\mathrm{Ae}$ & Kāo & Aua \\
\hline & & \\
\hline & & \\
\hline & & \\
\hline & & \\
& & \\
\hline
\end{tabular}


He aha ngā mea nui whakamahia ai e to kaiako e pā ana ki te whakawhanaunga i waenga i to karaehe?

These ideas came from students in Term 1.

Tohua te pouaka e hāngai tata ana ki ōu ake whakaaro.

he pai ki ahau ka menemene ana taku kaiako pangarau

he pai ki ahau inā ka whakaurua mai te taha katakata e taku kaiako pangarau

he pai ki ahau inā ka uiuitia a mātou mahi pai e taku kaiako pangarau

he mōhio taku kaiako pangarau ki taku ingoa

he pai ki ahau te mahi harikoa $\mathrm{i}$ roto $\mathrm{i}$ te mahi ako pangarau

he pai ki ahau tēnā ki te mahia ngā mahi pangarau uaua

he pai ki to mātou kaiako pangarau te ako i to mātou karaehe

he pai ki ahau inā, ka hōmaitia e to mātou kaiako pangarau ètahi kōwhiringa

he pai ki ahau mēna he ngāwari to mātou kaiako pangarau

he whakarongo taku kaiako pangarau ki āu

\begin{tabular}{|l|l|l|l|l|}
\hline $\begin{array}{c}\text { Strongly } \\
\text { Agree }\end{array}$ & Agree & Neutral & Disagree & $\begin{array}{c}\text { Strongly } \\
\text { Disagree }\end{array}$ \\
\hline & & & & \\
\hline & & & & \\
\hline & & & & \\
\hline & & & & \\
\hline & & & & \\
\hline & & & & \\
\hline & & & & \\
\hline & & & & \\
\hline
\end{tabular}


Me pēhea te whakahoki atu ki a koe i te oro whakautu a te kaiako e pā ana ki o mahi pangarau?

Ki o whakaaro, he mea nui ki a koe kia whai whakaaro mai to kaiako ki o mahi Pangarau? Hōmai he whakaaro ki o whakautu.

Ka nui te mihi mo ā kōutou whakautu i roto i tēnei pepa pātaitai. 
O le a le sa'o o nei fa'amatalaga mo oe?

Fa'ailoga le pusa e te iloa e fetaui ma lou manatu.

Ou te fiafia e a'oa'o i le numera

Ou te toaga e a'oa'o le numera

O lo'u faiaoga i le numera e mana'o e fesoasoani mai ia te a'u

Ou te fiafia i auala e fesoasoani mai ai lota faiaoga pe a ou sese

E fa'amalosi mai a'u e lo'u faiaoga ia ou taumafai atili

E fiafia le faiaoga pe a fesili atu le vasega ia te ia

E mana'o le faiaoga ia tauivi ma fa'amalolosi le vasega

E fa'aaloalo lo'u faiaoga ia te a'u

O lo'u faiaoga na te faailoa mai lona alofa ia te a'u

E tali e lo'u faiaoga o'u fesili tau i le mataupu

\begin{tabular}{|c|c|c|c|c|}
\hline $\begin{array}{c}\text { Taimi } \\
\text { uma }\end{array}$ & $\begin{array}{c}\text { I tele } \\
\text { o } \\
\text { taimi }\end{array}$ & $\begin{array}{c}\text { I nisi } \\
\text { taimi }\end{array}$ & $\begin{array}{c}\text { Le } \\
\text { tupu } \\
\text { so'o }\end{array}$ & $\begin{array}{c}\text { Le } \\
\text { tupu }\end{array}$ \\
\hline & & & & \\
\hline & & & & \\
\hline & & & & \\
\hline & & & & \\
\hline & & & & \\
\hline & & & & \\
\hline & & & & \\
\hline & & & & \\
\hline
\end{tabular}

Fa'ailoga le tali e latalata i ou lagona.

E iloa e le faiaoga lo'u lelei i numera.

E taua le fa'alelei atili o la'u numera i lenei tausaga

Ou te mautinoa e mafai ona ou faia le numera pe a ou taumafai loe Leai Leiloa

loe Leai Leiloa

loe Leai Leiloa 


\section{Filifili uma mea e fetaui ma oe.}

Ou te galue malosi i le numera aua

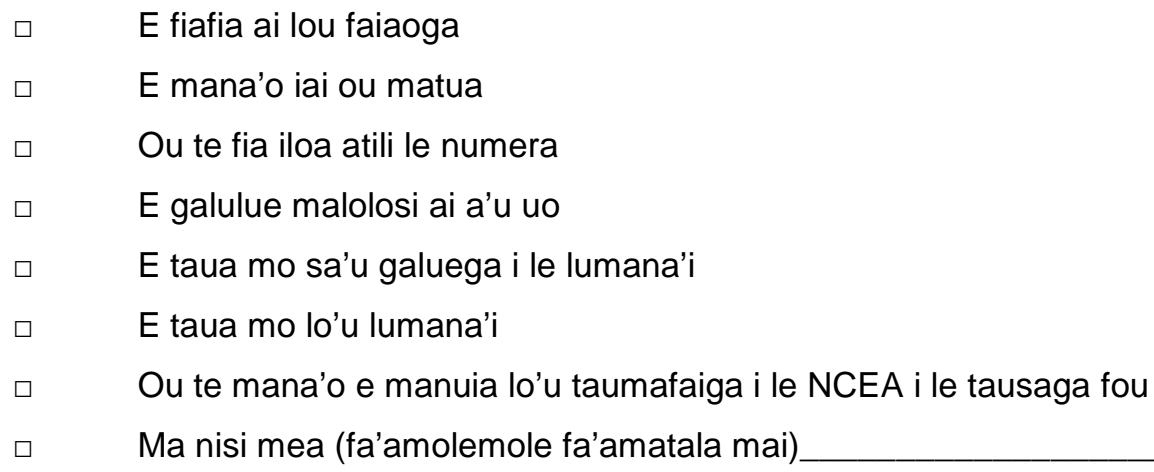

\section{Filifili uma mea e fetaui ma oe.}

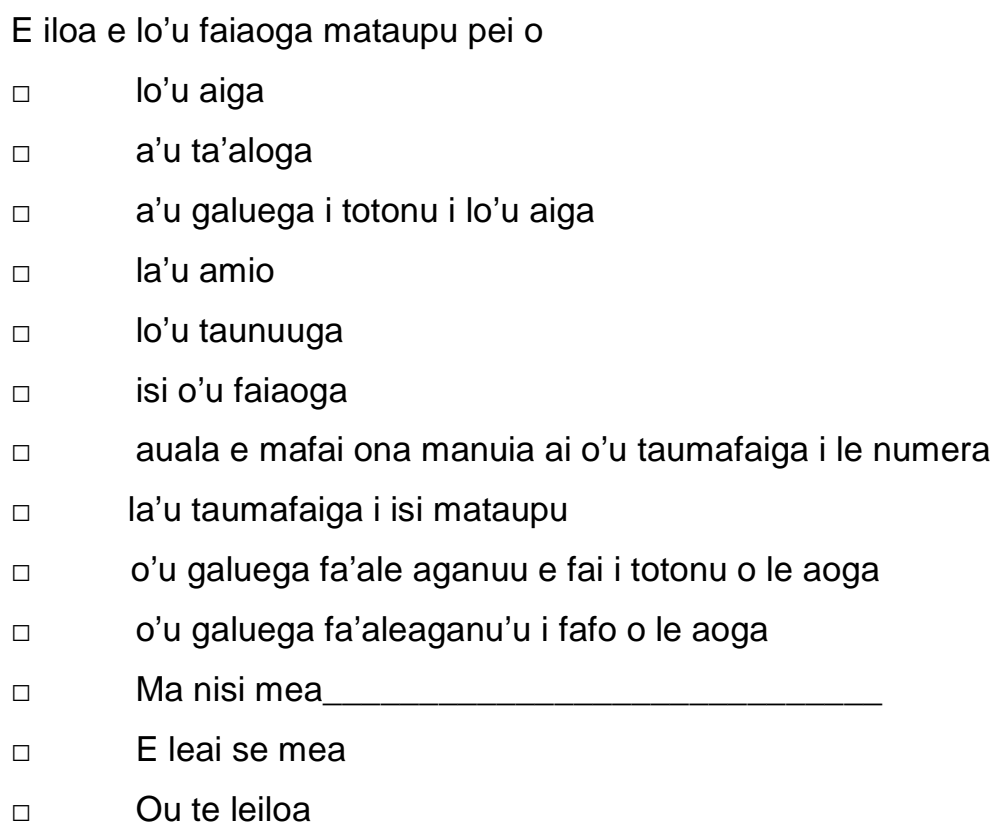

\section{Fa'ailoga le pusa e te iloa e fetaui ma lou manatu.}

E iloa e lo'u faiaoga lo'u nu'u

E iloa e lo'u faiaoga mataupu tau fa'aMāori

E iloa e lo'u faiaoga mataupu fa'apasefika

E fa'aogā e lo'u faiaoga fa'ata'ita'iga fa'aMāori, ma fa'apasefika i le matou vasega numera

\begin{tabular}{|l|l|l|}
\hline loe & Leai & $\begin{array}{c}\text { Lē } \\
\text { mautinoa }\end{array}$ \\
\hline & & \\
\hline & & \\
\hline & & \\
\hline & & \\
\hline
\end{tabular}


O ā mea e sili ona tāua e faia e lou faiaoga e mafanafana atili ai le mafutaga ma lou vasega?

These ideas came from students in Term 1.

Fa'ailoga le pusa e te iloa e fetaui ma lou manatu.

Ou te fiafia pe a mata'ata'ata le faiaoga Ou te fiafia pe a tausia le faiaoga

Ou te fiafia pe a tauia e lo'u faiaoga galuega lelei

E iloa e le faiaoga lo'u igoa

Ou te fiafia pe a matou fiafia i le a'oa'oina o le numera

Ou te fiafia pe a matou naunau i le numera

E fiafia le faiaoga e a'oa'o le vasega

Ou te fiafia pe a tu'u ni avanoa se tele mai le faiaoga mo matou

Ou te fiafia pe a onosa'i le faiaoga

E fa'alogo le faiaoga ia te a'u

\begin{tabular}{|l|l|l|l|l|}
\hline Taliainamalosi & Talia & $\begin{array}{c}\text { Fa'apea } \\
\text { lava }\end{array}$ & $\begin{array}{c}\text { Lē } \\
\text { taliaina }\end{array}$ & $\begin{array}{c}\text { Matuā } \\
\text { Iē } \\
\text { taliaina }\end{array}$ \\
\hline & & & & \\
\hline & & & & \\
\hline & & & & \\
\hline & & & & \\
\hline & & & & \\
\hline & & & & \\
\hline & & & & \\
\hline & & & & \\
\hline & & & & \\
\hline
\end{tabular}

Oā ni auala e te mana'o e ta'u atu ai e le faiaoga lou 'alu'alu i luma i le numera

E tauā le alofa o le faiaoga i lou a'oa'oina o le numera? Aiseā?

Fa'afetai tele mo le taliina o nei fesili. 


\section{Appendix 6: Third student questionnaires}

Name:

Date of birth:

Male Female

How true are each of the following statements for you?

Tick the box that most closely shows how you feel.

I like learning Maths

I work hard in Maths

My Maths teacher wants to help me learn

I like the way my teacher treats me when I make a mistake

My teacher encourages me to do my best

My teacher likes the class to ask questions

My teacher expects the class to work hard

My teacher treats me with respect

My teacher makes me feel they care about me

My teacher answers my questions about the work

\begin{tabular}{|l|l|l|l|l|}
\hline Always & $\begin{array}{c}\text { Most } \\
\text { of } \\
\text { the } \\
\text { time }\end{array}$ & $\begin{array}{c}\text { Some- } \\
\text { times }\end{array}$ & $\begin{array}{c}\text { Not } \\
\text { often }\end{array}$ & Never \\
\hline & & & & \\
\hline & & & & \\
\hline & & & & \\
\hline & & & & \\
\hline & & & & \\
\hline & & & & \\
\hline & & & & \\
\hline & & & & \\
\hline & & & & \\
\hline
\end{tabular}

Circle the answer that most closely shows how you feel.

My teacher knows how good I am at Maths

Yes No Don't know

It is important to me to improve my Maths skills this year

Yes No

I am certain I can do the Maths work in this class if I try

Yes No

Don't know

Tick all options that apply to you.

When I work hard in Maths it is because

I want to work well for my teacher

my parents expect me to

I want to learn the work

my friends work hard

it will be useful for my job in the future

it will be useful to me in life other than for a job

I want to be successful in NCEA next year

other (please explain) 
Tick all options that apply to you.

My maths teacher knows something about

my family

my sports

my family commitments

my personality

my successes

my other teachers

how well I can learn in maths

how well I learn in other subjects

my cultural activities at school

my cultural activities out of school

other

don't know

Tick the box that most closely shows how you feel.

My teacher gives me feedback about my maths progress

My teacher knows my ethnicity/culture

My teacher knows about the Māori world

My teacher knows about Pacific Nations things

My teacher uses Māori and Pacific Nations examples in Maths

\begin{tabular}{|c|c|c|}
\hline Yes & No & $\begin{array}{c}\text { I don't } \\
\text { know }\end{array}$ \\
\hline & & \\
\hline & & \\
\hline & & \\
\hline & & \\
\hline & & \\
\hline
\end{tabular}

Tick the box that most closely shows how you feel.

I like getting written feedback about my maths progress.

I like my teacher telling me about my maths progress.

I like my class to hear the teacher's comments about my work.

I am happy to ask my teacher about my maths progress.

\begin{tabular}{|l|l|l|l|l|}
\hline $\begin{array}{c}\text { Strongly } \\
\text { Agree }\end{array}$ & Agree & Neutral & Disagree & $\begin{array}{c}\text { Strongly } \\
\text { Disagree }\end{array}$ \\
\hline & & & & \\
\hline & & & & \\
\hline & & & & \\
\hline & & & & \\
\hline
\end{tabular}


If you can, please list things special to your own culture.

Tick the box that most closely shows how you feel.

\begin{tabular}{|c|c|c|c|c|c|}
\hline \multirow[b]{2}{*}{$\begin{array}{l}\text { Our teacher has high expectations of our } \\
\text { maths learning. }\end{array}$} & $\begin{array}{l}\text { Strongly } \\
\text { Agree }\end{array}$ & Agree & $\begin{array}{l}\text { Don't } \\
\text { know }\end{array}$ & Disagree & $\begin{array}{l}\text { Strongly } \\
\text { Disagree }\end{array}$ \\
\hline & & & & & \\
\hline \multicolumn{6}{|l|}{ Our teacher praises us for good work. } \\
\hline \multicolumn{6}{|l|}{ Our teacher is patient. } \\
\hline Our teacher believes we can achieve. & & & & & \\
\hline Our teacher knows our class well. & & & & & \\
\hline
\end{tabular}

Some schools show that students' ethnicities/cultures are important and others don't. Place a tick in the box or on the line to show how your school treats students' ethnicities/cultures.

I don't know if students' cultures are important to my school.

Students'

Students'

cultures are

very important at

our school cultures are

not important at all

at our school

If you can, please give an example of how your school shows this. 
Some teachers show that students' ethnicities/cultures are important and others don't. Place a tick in the box or on the line to show how your teacher thinks about students' ethnicities/cultures.

I don't know if students' cultures are important to my teacher.

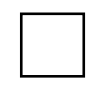

Students'

cultures are very

important to our

teacher
Students'

cultures are not important at all to our teacher

If you can, please give an example of how your teacher shows this.

It is important to me that our teacher cares about our ethnicities/cultures.

My ethnicity/culture is not relevant when I am at school.

My ethnicity/culture is not relevant when I am learning maths.

\begin{tabular}{|l|l|l|l|l|}
\hline $\begin{array}{l}\text { Strongly } \\
\text { Agree }\end{array}$ & Agree & Neutral & Disagree & $\begin{array}{l}\text { Strongly } \\
\text { Disagree }\end{array}$ \\
\hline & & & & \\
\hline & & & & \\
\hline & & & & \\
\hline
\end{tabular}

Do you think it is important for your maths learning that your teacher cares about students' cultures?

Yes

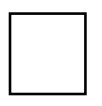

No

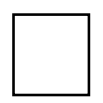

Don't know

Please give reasons for your answer 
Every person is a part of lots of different groups. Below are some groups you might belong to.

On the first diagram please show how important each of these groups is to you in your life.

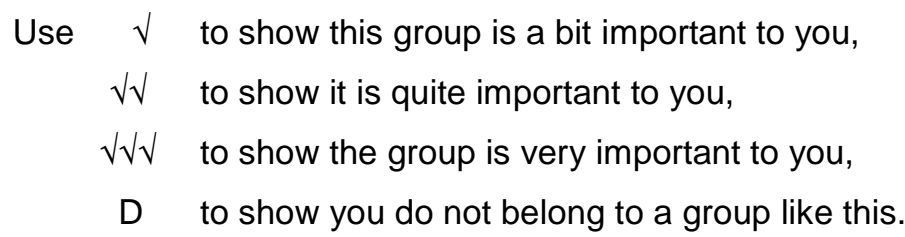

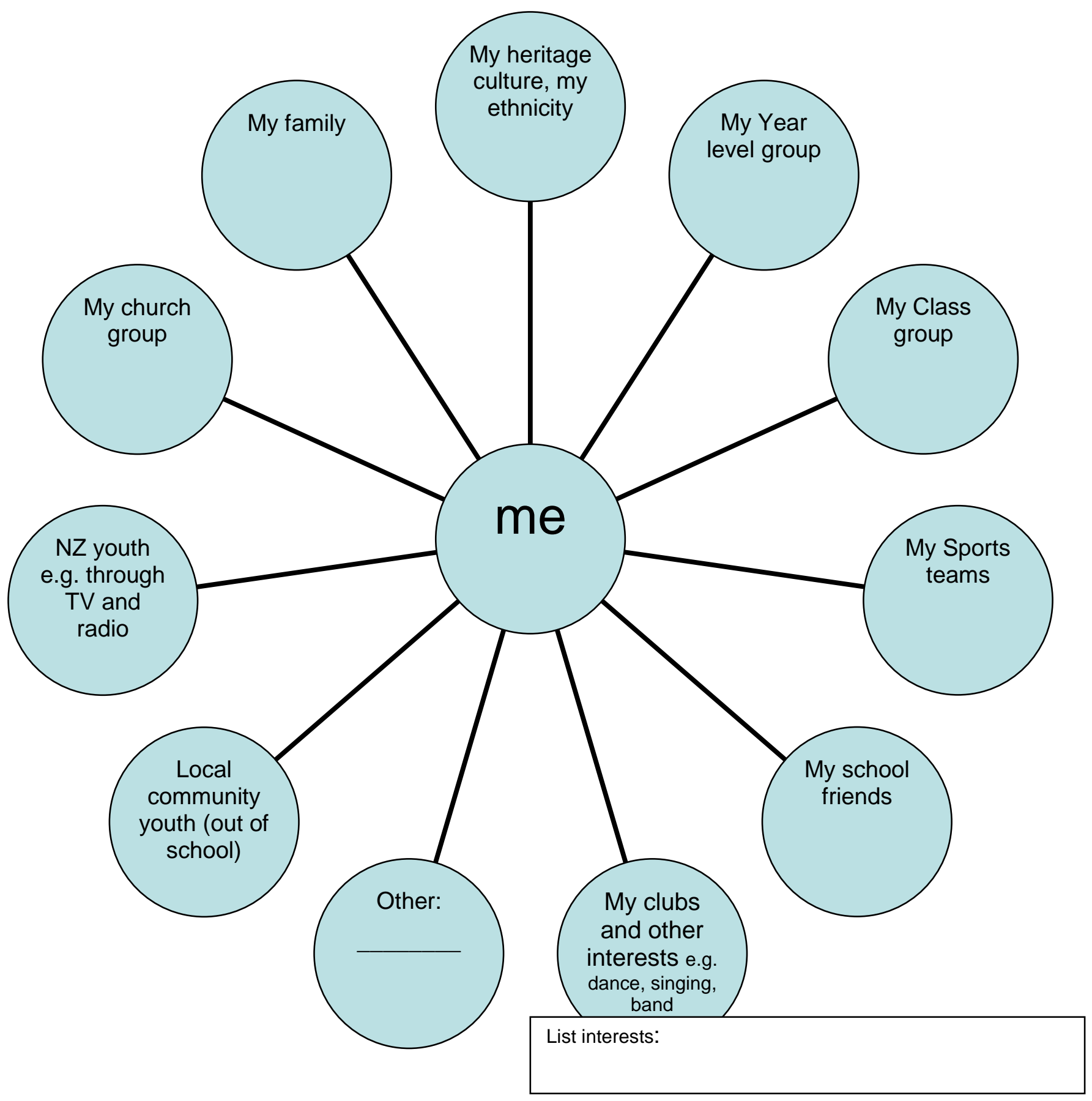


On the second diagram show how important each of these groups is to you when you are learning maths.

Use $\sqrt{ }$ to show this group is a bit important to you when you are learning maths, $\checkmark \sqrt{ }$ to show it is quite important to you when you are learning maths, $\sqrt{ } \sqrt{ }$ to show the group is very important to you when you are learning maths,

$\mathrm{X}$ to show the group is not relevant when you are learning maths.

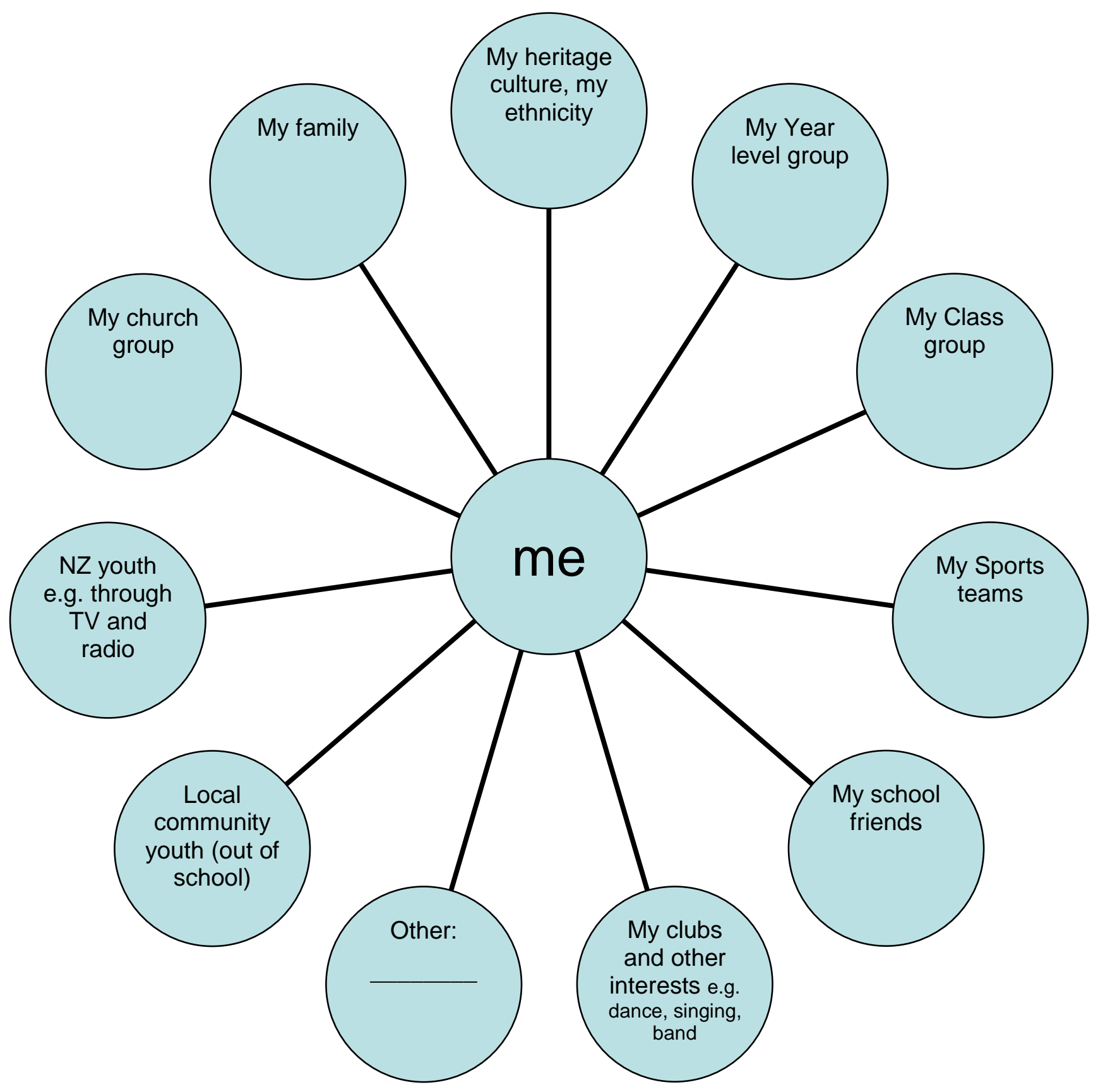


Did you notice any surprising results when the data from Term 1 and 2 were shared with the class?

Yes

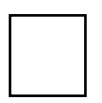

No

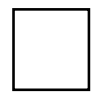

Don't know

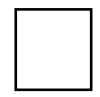

If so, what were they? Why did they surprise you?

Did you notice any differences in your maths lessons after the data from Term 1 and 2 were shared with the class?

Yes

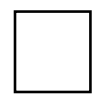

No

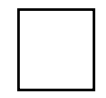

Don't know

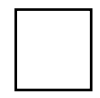

If so, what were they? What did you think about this?

Thank you very much for your help in this project and very best wishes for your maths study in the future. 
Ingoa:

Pēhea te tika o ngā rārangi nei e pā ana ki a koe?

Tohua te pouaka e hāngai tata ana ki ōu ake whakaaro.

He pai ki au te whakamātau Pangarau.

He kaha taku mahi i te Pangarau.

E hiahia ana taku kaiako Pangarau ki te awhi i au i roto i aku mahi.

He pai ki au te āhuatanga whakahaere a taku kaiako i te wā hē aku mahi.

He tautoko taku kaiako kia eke aku mahi ki te taumata e taea ai e au.

He pai ki te kaiako, kia pātaitai ngā akonga.

He tūmanako na te kaiako ka mahi kaha ngā akonga.

He manaaki nui taku kaiako i au.

Ka uru mai te whakaaro nei na, he aro pai taku kaiako mōku.

Ngā whakautu a taku kaiako ki aku pātai mo te mahi.

\begin{tabular}{|l|l|l|l|l|}
\hline $\begin{array}{c}\text { i ngā } \\
\text { wā } \\
\text { katoa }\end{array}$ & $\begin{array}{c}\text { i te } \\
\text { nuinga } \\
\text { o te wā }\end{array}$ & $\begin{array}{c}\text { ètahi } \\
\text { wā }\end{array}$ & $\begin{array}{c}\text { iti nei } \\
\text { te wā }\end{array}$ & kore \\
\hline & & & & \\
\hline & & & & \\
\hline & & & & \\
\hline & & & & \\
\hline & & & & \\
\hline & & & & \\
\hline & & & & \\
\hline & & & & \\
\hline & & & & \\
\hline
\end{tabular}

\section{Porowhitatia te whakautu e hāngai tata ana ki ōu ake whakaaro.}

He mōhio taku kaiako ki tōku pai ki te mahi Pangarau.

He mea nui ki au ki te hiki ake i taku mōhio ki te mahi

Pangarau i tēnei tau.

Me taku mōhio anō, ki te ngana atu au, ka taea e au ngā mahi Pangarau i roto i te akomanga.

$\begin{array}{lll}\text { Ae } & \text { Kāo } & \text { Aua } \\ \text { Ae } & \text { Kāo } & \text { Aua } \\ \text { Ae } & \text { Kāo } & \text { Aua }\end{array}$


Tohua katoatia ngā ara whiringa e pā ana ki a koe.

Ina kaha taku mahi Pangarau, na te mea

- he hiahia kia pai aku mahi mo taku kaiako

- he wawata na aku mātua kia.....

口 he hiahia nōku ki te mahi

- he mahi kaha na ōku hoa

ka whai hua taku mahi a ngā wa e heke mai nei

- he hua kei roto i tēnei mōku, tēra i tua atu o te mahi

- kei te hiahia ahau kia whai hua au i te NCEA a te tau e tū mai nei

口 Ėtahi atu (tēna whakamaaramatia)

Tohua katoatia ngā ara whiringa e pā ana ki a koe.

He mōhio taku kaiako pangarau ki ētahi mea e pā ana ki:

\ taku whānau

口 aku mahi tākaro

๖ tāku e ngākau nui ana ki taku whānau

口 tōku tuakiritanga

- aku mahi angitu

- taku pai ki te mahi pangarau

- taku pai ki te ako i ètahi atu marau

- aku mahi taha tikanga i te kura

- aku mahi taha tikanga i waho o te kura

口 ētahi atu

- Kāore he pānga ki ēnei

口 Kāore au e mōhio

Tohua te pouaka e hāngai tata ana ki ōu ake whakaaro.

ka whakahoki urupare mai taku kaiako i aku mahi nekenga pangarau.

he mōhio taku kaiako ki taku iwi/taha tikanga

he mōhio taku kaiako ki te Ao Māori

he mōhio taku kaiako ki ngā pānga a ngā iwi o Te Moana Nui ā Kiwa

whakamahia ai e taku kaiako ētahi tauira pangarau a te Māori, me a ngā iwi te Moana Nui ā Kiwa

\begin{tabular}{|l|l|l|}
\hline $\mathrm{Ae}$ & Kāo & Aua \\
\hline & & \\
\hline & & \\
\hline & & \\
\hline & & \\
\hline & & \\
\hline
\end{tabular}


Tohua te pouaka e hāngai tata ana ki ōu ake whakaaro.

He pai ki ahau ki te whiwhi whakautu-atuhi mo aku mahi nekenga pangarau.

He pai ki ahau ki te whakautu uiui mai taku kaiako mo aku mahi nekenga pangarau.

He pai ki ahau kia ranonga e taku karaehe ngā kōrero a te kaiako e pā ana ki aku mahi nekenga pangarau.

He mea koa ki ahau ki te pātai ki taku kaiako mo aku mahi neke pangarau.

\begin{tabular}{|l|l|l|l|l|}
\hline $\begin{array}{c}\text { Strongly } \\
\text { Agree }\end{array}$ & Agree & Neutral & Disagree & $\begin{array}{c}\text { Strongly } \\
\text { Disagree }\end{array}$ \\
\hline & & & & \\
\hline & & & & \\
\hline & & & & \\
\hline & & & & \\
\hline & & & & \\
\hline
\end{tabular}

Mehemea ka taea e koe, tēnā koa whakarārangihia ētahi āhuatanga ki ōu tikanga.

Tohua te pouaka e hāngai tata ana ki ōu ake whakaaro.

He teitei ngā tūmanako a to mātou kaiako mo a mātou mahi ako pangarau.

Ka whakamihi to mātou kaiako ki a mātou mahi pai.

He ngākau hūmarie to mātou kaiako.

He whakapono to mātou kaiako ka taea e mātou ngā taumata whāinga.

He mātau mōhio to mātou kaiako ki to mātou rōpu karaehe.

\begin{tabular}{|l|l|l|l|l|}
\hline $\begin{array}{c}\text { Strongly } \\
\text { Agree }\end{array}$ & Agree & $\begin{array}{c}\text { Don't } \\
\text { know }\end{array}$ & Disagree & $\begin{array}{c}\text { Strongly } \\
\text { Disagree }\end{array}$ \\
\hline & & & & \\
\hline & & & & \\
\hline & & & & \\
\hline & & & & \\
\hline & & & & \\
\hline
\end{tabular}


He whakanui ètahi kura i te taha iwi/tikanga o te ākonga. Kaare ètahi.

Tohua he whakaae, he rārangi rānei ki roto i te pouaka, e whakaatu ana he pēhea te manaaki a to kura i ngā tikanga/iwi o te ākonga.

Kāore au e mōhio mehemea he aronuitia te taha iwi/tikanga o nga ākonga e to mātou kura.

He mea tino nui

ngā tikanga o te

Kāore ngā tikanga o

ākonga ki to te ākonga e aronuitia

mātou kura. ana e to mātou kura.

Ki te taea e koe, tēnā koa whakaaturia mai he tauira a to kura mo tēnei.

He whakanui ētahi kaiako i te taha iwi/tikanga o te ākonga. Kaare ētahi.

Tohua he whakaae, he rārangi rānei e whakaatu ana he pēhea te manaaki a to kaiako i ngā tikanga/iwi o te ākonga.

Kāore au e mōhio mehemea he aronui to mātou kaiako ki te tuakiritanga/tikanga o ngā ākonga

He mea tino nui

Kāore ngā tikanga o

ngā tikanga o te

te ākonga e

ākonga.ki to

aronuitia ana e to

mātou kaiako.

mātou kaiako.

Ki te taea e koe, tēnā koa whakaaturia mai he tauira a to kaiako mo tēnei. 
He mea nui ki ahau kia aronui mai to mātou kaiako ki to mātou taha iwi/tikanga.

Kāore taku taha iwi/tikanga i hängai i te wā kei te kura ahau.

Kāore taku taha iwi/tikanga i hāngai i te wā i ähau kei te ako pangarau.

\begin{tabular}{|l|l|l|l|l|}
\hline $\begin{array}{c}\text { Strongly } \\
\text { Agree }\end{array}$ & Agree & Neutral & Disagree & $\begin{array}{c}\text { Strongly } \\
\text { Disagree }\end{array}$ \\
\hline & & & & \\
\hline & & & & \\
\hline & & & & \\
& & & & \\
\hline
\end{tabular}

Ki o whakaaro, he mea nui i roto i o mahi pangarau mehemea ka kaingākau mai to kaiako ki te taha tikanga-ā-iwi o ngā ākonga?

$\mathrm{Ae}$

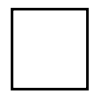

Kāo

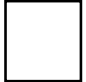

Aua

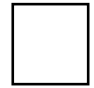

Hōmai he whakautu 
He maha ngā pānga o ia tangata kei roto i ngā rerenga rōpū. Whai iho ko ètahi rōpū kei roto pea koe.

I te mahere tuatahi, tēnā tohua ko ēhea o ēnei rōpū e aronui atu ana koe.

Tohua $\quad \sqrt{ }$ he iti noa to aronga ki tēnei rōpū.

$\sqrt{ } \sqrt{ }$ he mea nui to aronga ki tēnei röpü.

$\sqrt{ } \sqrt{ }$ he tino nui to aronga ki tēnei rōpū.

D kaare koe i hāngai atu ki te rōpū pēnei.

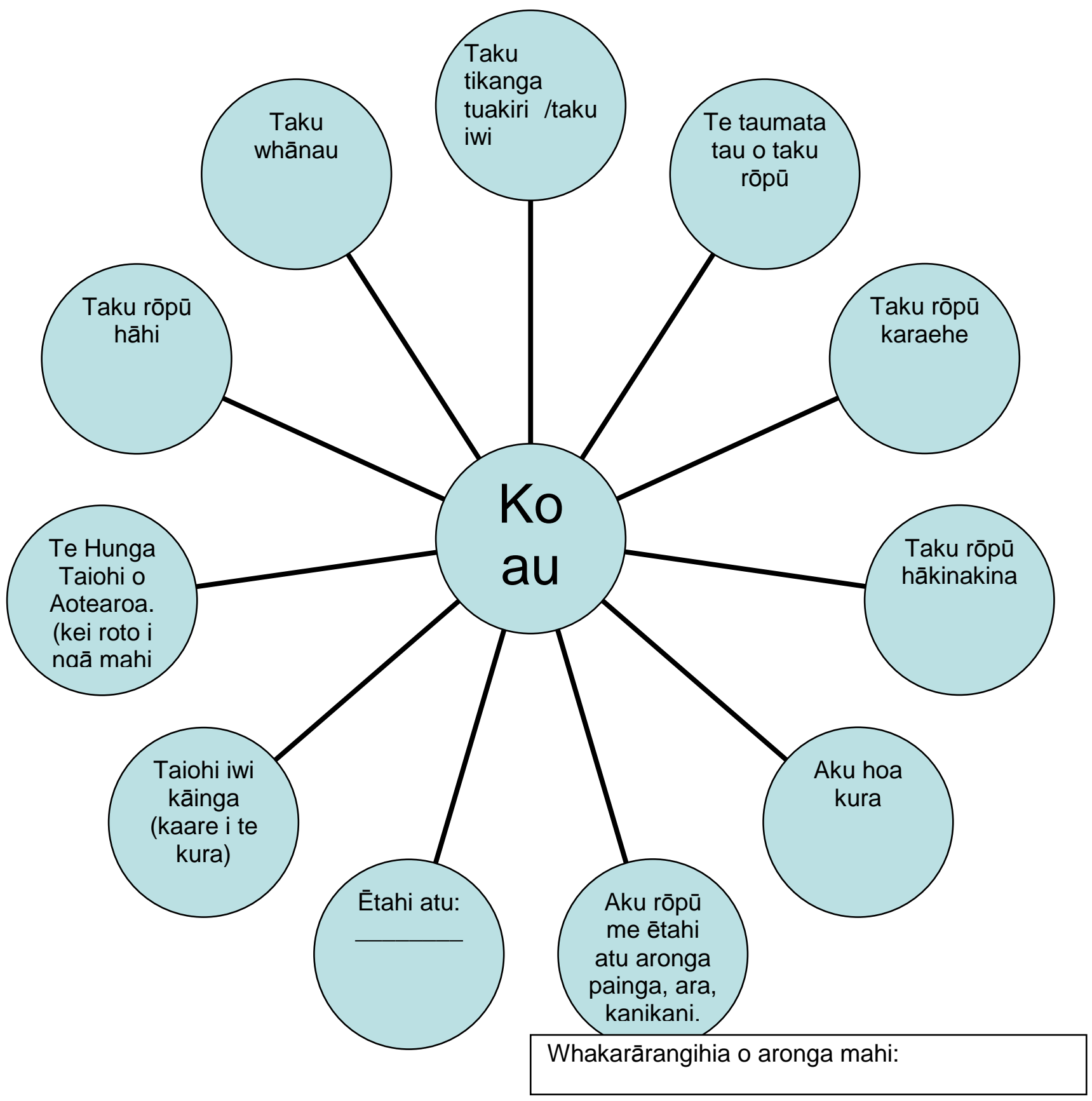


I te mahere tuarua, tēnā whakaaturia mai ko tēhea o ēnei rōpū te mea nui ki a koe i te wā o to mahi akoranga pangarau.

Tohua tēnei $\sqrt{ }$ he iti noa to aronga ki tēnei rōpū $i$ te wā o to mahi pangarau.

$\sqrt{ } \sqrt{ }$ he mea nui to aronga ki tēnei rōpū i te wā o to mahi pangarau.

$\checkmark \sqrt{ } \sqrt{ }$ he tino nui to aronga ki tēnei rōpū i te wā o to mahi pangarau.

X Kāore tēnei rōpū i hāngai ki a koe i te wā o to mahi pangarau.

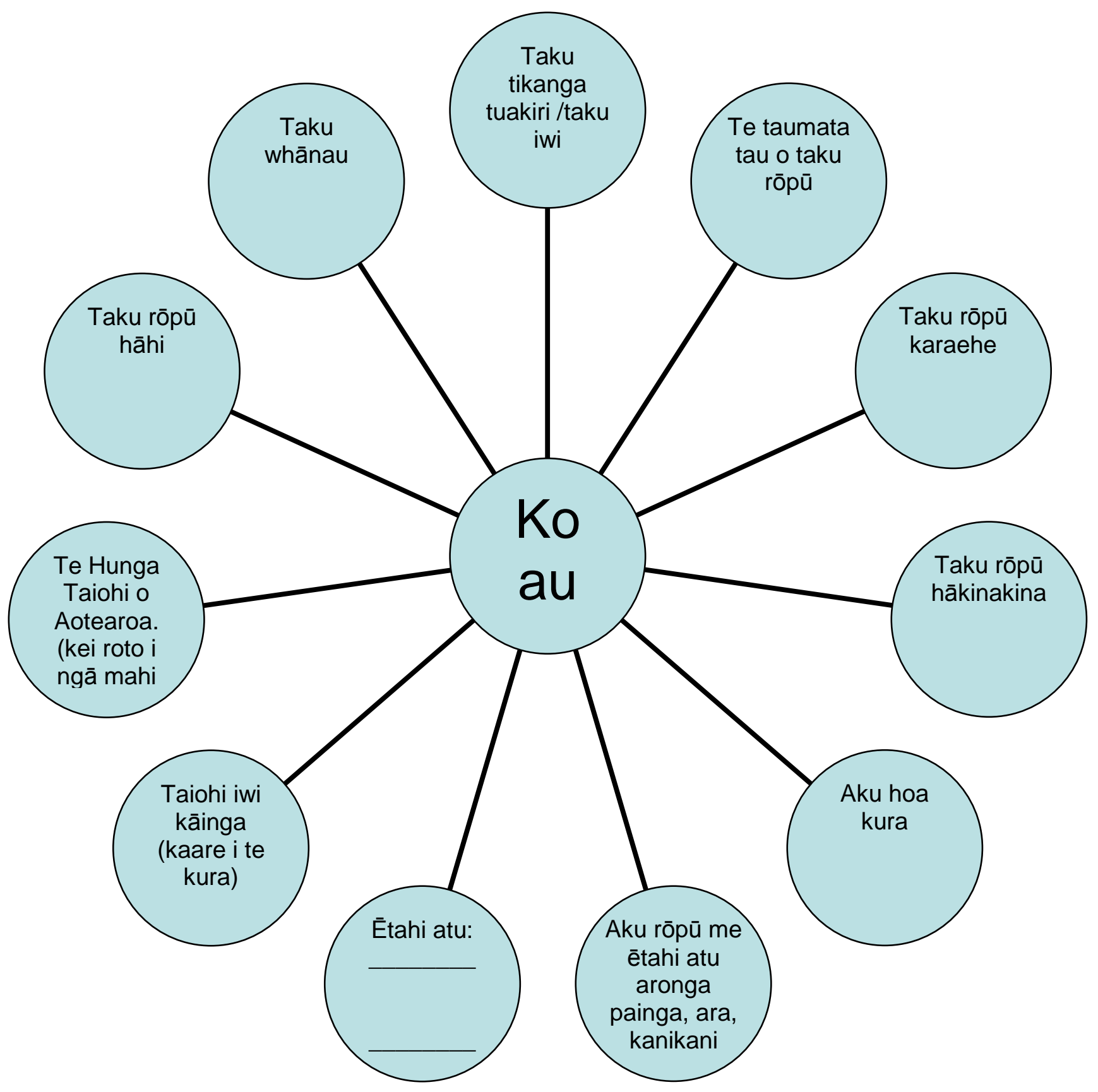


I kite anō koe i ētahi āhuatanga rerekē mai i ngā kohikohinga o te wāhanga tuatahi ki te tuarua i te wā i tirohia e to karaehe?

$\mathrm{Ae}$

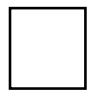

Kāo

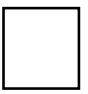

Aua

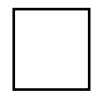

Mehemea ae, he aha? He aha i tuki ohorere ai ki a koe?

I kite anō koe i ētahi rerekētanga i roto i o mahi pangarau, i muri iho o te wāhanga tuatahi ki te tuarua i te wā i tirohia e to karaehe?

$\mathrm{Ae}$

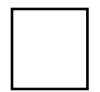

Kāo

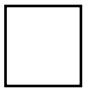

Aua

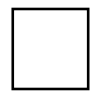

Mehemea ae, he aha? He aha o whakaaro mo tēnei?

Ngā mihi nui ki a koe mo to āwhina i runga i tēnei kaupapa, otirā, ka haere tonu ngā mihi ki o mahi Pangarau a ngā tau e tū mai nei. 
O le a le sa'o o nei fa'amatalaga mo oe?

Fa'ailoga le pusa e te iloa e fetaui ma lou manatu.

Ou te fiafia e a'oa'o i le numera

Ou te toaga e a'oa'o le numera

O lo'u faiaoga i le numera e mana'o e fesoasoani mai ia te a'u

Ou te fiafia i auala e fesoasoani mai ai lota faiaoga pe a ou sese

E fa'amalosi mai a'u e lo'u faiaoga ia ou taumafai atili

E fiafia le faiaoga pe a fesili atu le vasega ia te ia

E mana'o le faiaoga ia tauivi ma fa'amalolosi le vasega

E fa'aaloalo lo'u faiaoga ia te a'u

O lo'u faiaoga na te faailoa mai lona alofa ia te a'u

E tali e lo'u faiaoga o'u fesili tau i le mataupu

\begin{tabular}{|c|c|c|c|c|}
\hline $\begin{array}{l}\text { Taimi } \\
\text { uma }\end{array}$ & $\begin{array}{c}\text { I tele } \\
0 \\
\text { taimi }\end{array}$ & $\begin{array}{l}\text { I nisi } \\
\text { taimi }\end{array}$ & $\begin{array}{l}\text { Le } \\
\text { tupu } \\
\text { so'o }\end{array}$ & Le tupu \\
\hline & & & & \\
\hline & & & & \\
\hline & & & & \\
\hline & & & & \\
\hline & & & & \\
\hline & & & & \\
\hline & & & & \\
\hline & & & & \\
\hline & & & & \\
\hline & & & & \\
\hline & & & & \\
\hline & & & & \\
\hline
\end{tabular}

Fa'ailoga le tali e latalata i ou lagona.

E iloa e le faiaoga lo'u lelei i numera.

E taua le fa'alelei atili o la'u numera i lenei tausaga

Ioe Leai Leiloa

loe Leai Leiloa

Ou te mautinoa e mafai ona ou faia le numera pe a ou taumafai loe Leai Leiloa 


\section{Filifili uma mea e fetaui ma oe.}

Ou te galue malosi i le numera aua

$\square \quad$ E fiafia ai lou faiaoga

口 E mana'o iai ou matua

口 Ou te fia iloa atili le numera

$\quad$ E galulue malolosi ai a'u uo

$\quad$ E taua mo sa'u galuega i le lumana'i

$\quad$ E taua mo lo'u lumana'i

口 Ou te mana'o e manuia lo'u taumafaiga i le NCEA i le tausaga fou

Ma nisi mea (fa'amolemole fa'amatala mai)

\section{Filifili uma mea e fetaui ma oe.}

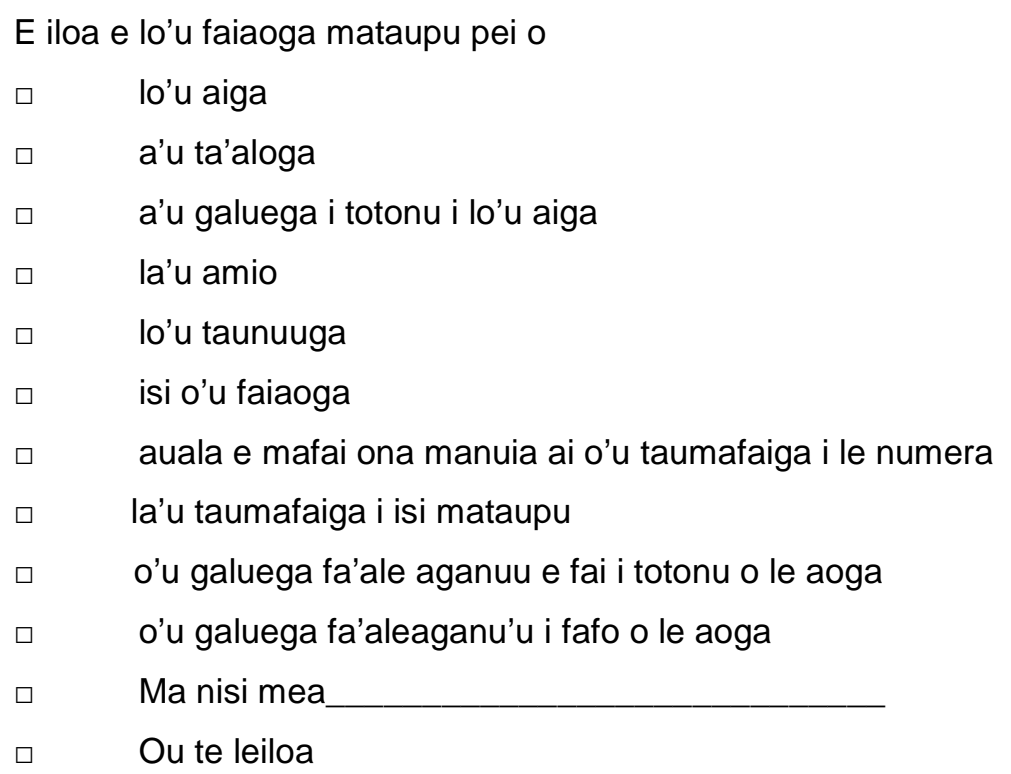


Fa'ailoga le pusa e te iloa e fetaui ma lou manatu.

E ta'u mai e le faiaoga lo'u taumafaiga i le numera

E iloa e lo'u faiaoga lo'u nu'u

E iloa e lo'u faiaoga mataupu tau fa'aMāori

E iloa e lo'u faiaoga mataupu fa'apasefika

E fa'aogā e lo'u faiaoga fa'ata'ita'iga fa'aMāori, ma fa'apasefika i le matou vasega numera

\begin{tabular}{|l|l|l|}
\hline Ioe & Leai & $\begin{array}{c}\text { Lē } \\
\text { mauti } \\
\text { noa }\end{array}$ \\
\hline & & \\
\hline & & \\
\hline & & \\
\hline & & \\
\hline & & \\
\hline
\end{tabular}


Fa'ailoga le pusa e te iloa e fetaui ma lou manatu.

Ou te fiafia pe a aumai se lipoti tusia o le faiaoga e iuga i la'u taumafai i le numera

Ou te fiafia pe a talanoa mai le faiaoga i lo'u taumafaiga i le numera

Ou te fiafia e fa'alogo mai le vasega pe a talanoa mai le faiaoga i lo'u taumafaiga i le numera

Ou te fiafia e fesili i le faiaoga lo'u taumafaiga i le numera

\begin{tabular}{|l|l|l|l|l|}
\hline Taliainamalosi & Talia & $\begin{array}{c}\text { Fa'apea } \\
\text { lava }\end{array}$ & $\begin{array}{c}\text { Lē } \\
\text { taliaina }\end{array}$ & $\begin{array}{c}\text { Matuā } \\
\text { lē } \\
\text { taliaina }\end{array}$ \\
\hline & & & & \\
\hline & & & & \\
\hline & & & & \\
& & & & \\
\hline & & & & \\
\hline
\end{tabular}

Afai e mafai fa'amolemole tusi mai ni fa'ata'ita'iga o lau aganu'u

Fa'ailoga le pusa e te iloa e fetaui ma lou manatu.

O le mātou faiaoga e fa'amanuia mai mätou galuega lelei e faia i le numera

E faali mai e le faiaoga lona fiafia pe afai e lelei mātou galuega

E onosa'i le faiaoga

E talitonu le faiaoga e mafai ona matou faia so'o se mea

E iloa e le faiaoga le mātou vasega

\begin{tabular}{|l|l|l|l|l|}
\hline Taliainamalosi & Talia & $\begin{array}{c}\text { Fa'apea } \\
\text { lava }\end{array}$ & $\begin{array}{c}\text { Lēe } \\
\text { taliaina }\end{array}$ & $\begin{array}{c}\text { Matuā } \\
\text { Iē } \\
\text { taliaina }\end{array}$ \\
\hline & & & & \\
\hline & & & & \\
\hline & & & & \\
\hline & & & & \\
\hline & & & & \\
\hline & & & & \\
\hline
\end{tabular}


E tāua le aganu'u o se tagata i totonu o aoga? A'o isi aoga e lē taua le aganu'u Fa'ailoa mai po'o fea le pusa e moni mo oe, e fa'ailoa mai ai pe fa'apefea ona fa'atino lau aganu'u i totonu o le aoga

Ou te lē mautinoa pe tāua le aganu'u o tamaiti aoga i le mätou aoga

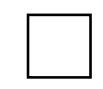

\begin{tabular}{|rr|}
\hline E tāua le & E lē tāua le \\
aganu'u o & aganu'u o tamaiti \\
le tamaitiiti & aoga i le mātou \\
aoga i le & aoga
\end{tabular}

mātou aoga

Afai e mafai, ta'u mai pe fa'apefea ona fa'aali e le aoga le tāua o le aganu'u

O nisi faiaoga e faaali mai le tāua o le aganu'u o le tamaititi aoga, a'o isi aoga e le fa'aalia mai

Fa'ailoa mai po'o fea e moni mo oe e fa'ailoa mai ai po'o ā mafaufauga o le faiaoga i le aganu'u o se tamaititi

Ou te lē mautinto pe tāua le aganu'u o se tamaititi i lo'u faiaoga

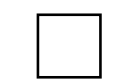

E tāua le

E lē tāua le

aganu'u o le

aganu'u o le

tamaititi i lo'u

tamaitiiti aoga i le

faiaoga

mātou faiaoga

Afai e mafai, tusi mai ni fa'ata'ita'iga 
E taua ia te a'u le faaaloalo o lo'u faiaoga i mātou aganu'u

E lē tāua la'u aganu'u pe a ou ulufale i le aoga

E lē tāua la'u aganu'u pe a a'oa'oina le numera

\begin{tabular}{|l|c|c|c|c|}
\hline $\begin{array}{c}\text { Taliaina } \\
\text { malosi }\end{array}$ & Talia & $\begin{array}{c}\text { Fa'apea } \\
\text { lava }\end{array}$ & $\begin{array}{c}\text { Lē } \\
\text { taliaina }\end{array}$ & $\begin{array}{c}\text { Matuā } \\
\text { Īe } \\
\text { taliaina }\end{array}$ \\
\hline & & & & \\
\hline & & & & \\
\hline & & & & \\
\hline
\end{tabular}

E te talitonu e tāua ona fa'ailoa e lou faiaoga numera le fa'aaloalo i lau aganu'u

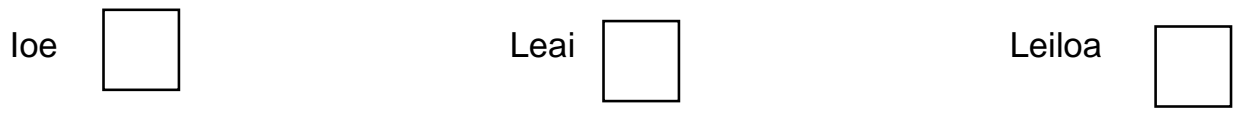

Aisea? 
E to'atele fa'alāpotopotoga e auai ai le tagata. Lalo ifo o loo iai nisi o fa'alāpotopotoga e te auai ai.

Fa'aogā le ata e fa'aali mai ai le tāua o fa'alāpotopotoga i lou olaga

$\sqrt{ } \quad E$ fa'aali mai e la'itiiti le tāua ia te oe

$\sqrt{ } \sqrt{ } \quad E$ fa'aali mai e tāua ia te oe

$\sqrt{ } \sqrt{ } \quad E$ fa'aali mai e telē le tāua ia te oe

D E fa'aali mai ai e te lē auai i lenā fa'alāpotopotoga

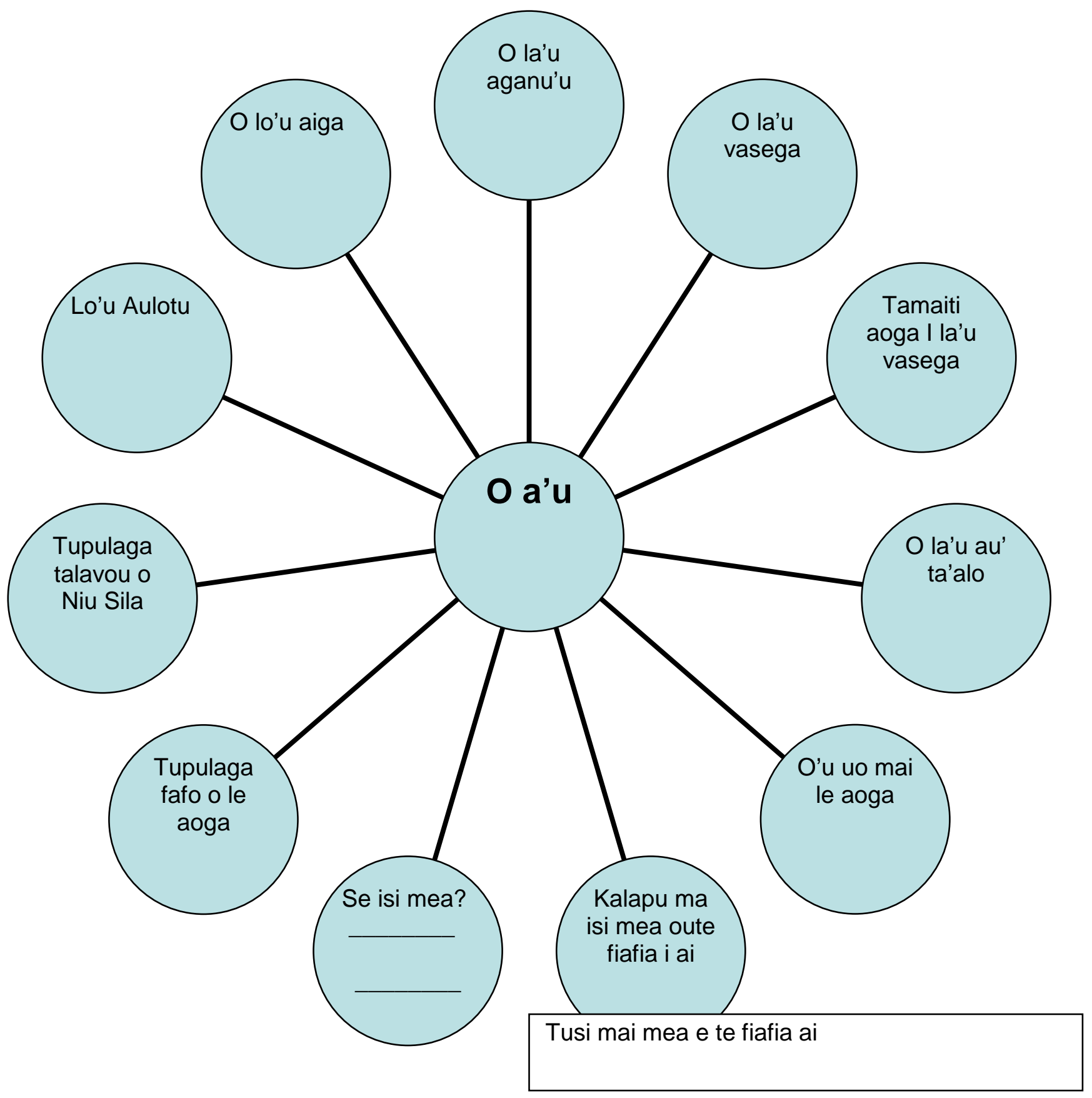


I le ata lona lua fa'aali mai le tāua o ia fa'alapotopotoga pe a a'oina lau numera
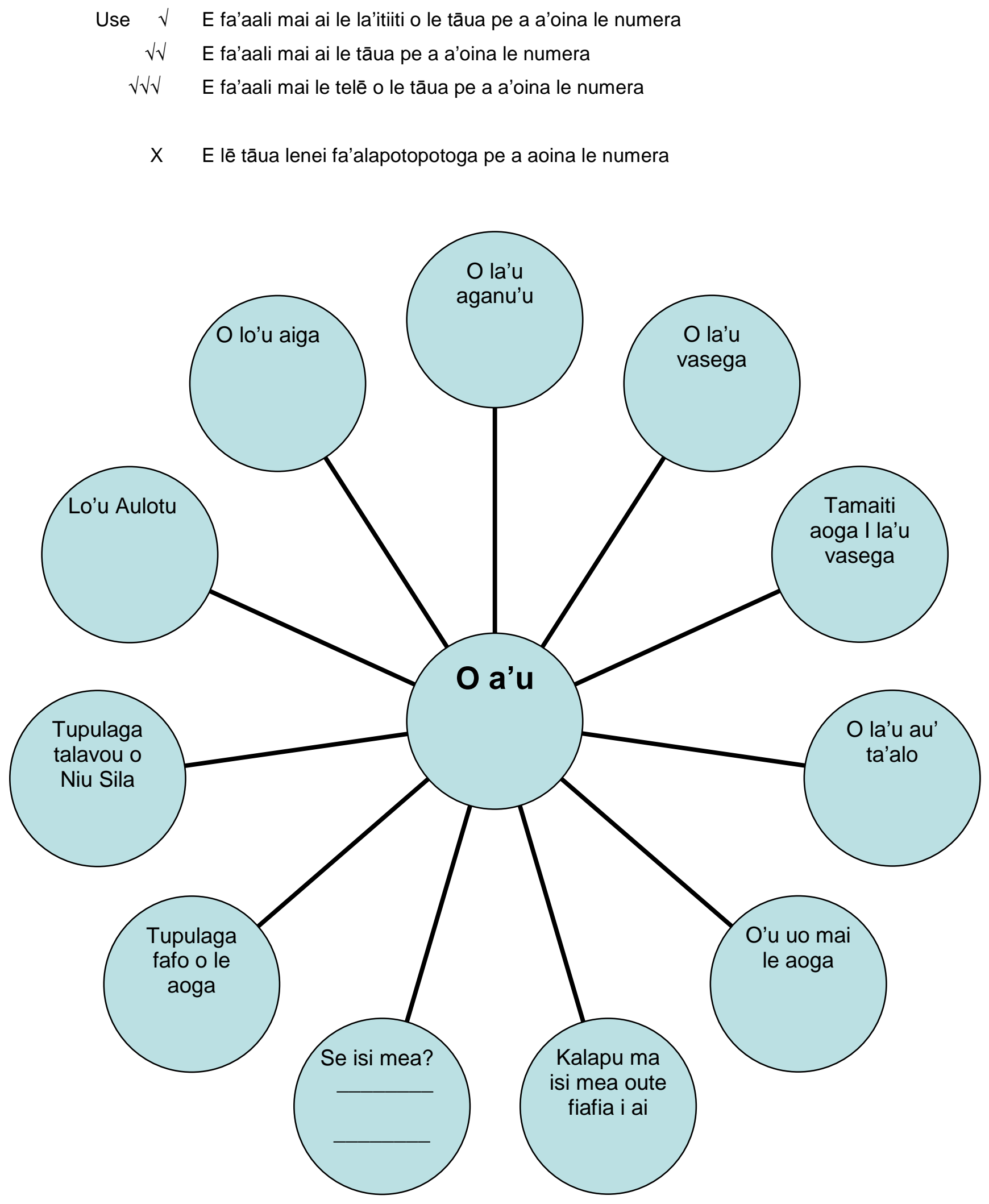
I le vaaiga i su'esu'ega o le kuata muamua ma le lua o iai ni mea e te fia fa'aali pe ta'ua i le vasega?

loe

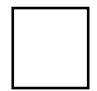

Leai

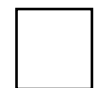

Leiloa

Afai o lo'o iai. O le ā? Aisea?

$\mathrm{Na}$ e silafia ni eseesega i totonu o lou vasega numera ina ua uma ona fa'aali atu su'esu'ega o le kuata muamua ma le lua
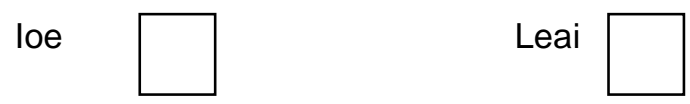

Leiloa

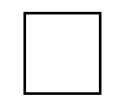

Afai sa iai o le ā? O ā ni ou mafaufauga?

Fa'afetai lava mo lou fesoasoani mai i lenei su'esu'ega. la manuia lou taumafai i le numera mo le lumana'i. 


\section{Appendix 7: Initial teacher questionnaire}

Teacher Questionnaire: Term 1

Name:

How true are each of the following statements for you?

Tick the box which most closely shows how you feel.

I like teaching Year 10 mathematics.

I expect students in this class to work hard.

I like to link maths learning to students' interests.

Knowing students' names

\begin{tabular}{|l|l|l|l|l|}
\hline Always & $\begin{array}{l}\text { Most of } \\
\text { the time }\end{array}$ & Sometimes & Not often & Never \\
\hline & & & & \\
\hline & & & & \\
\hline & & & & \\
\hline & & & & \\
\hline
\end{tabular}

Tick the box to show how strongly you agree with the following statements:

I believe effective teacher-student relationships are essential for good maths learning for this class.

I believe the teacher has a strong influence on students' attitudes to maths learning.

I believe the teacher has a strong influence on students' maths achievement.

I believe maths achievement is important for this class.

I know the maths abilities of all students in this class.

I believe all students in this class can succeed with Year 10 maths.

I believe all students in this class want to learn Year 10 maths.

\begin{tabular}{|l|l|l|l|l|}
\hline $\begin{array}{l}\text { Strongly } \\
\text { Agree }\end{array}$ & Agree & Neutral & Disagree & $\begin{array}{l}\text { Strongly } \\
\text { Disagree }\end{array}$ \\
\hline & & & & \\
\hline & & & & \\
\hline & & & & \\
\hline & & & & \\
\hline & & & & \\
\hline & & & & \\
\hline & & & & \\
\hline & & & & \\
\hline
\end{tabular}


Three students in this class who I think are likely to achieve well in maths in this class are

\begin{tabular}{|l|l|}
\hline Student's name & Main reason for your expectation \\
\hline & \\
\hline & \\
\hline & \\
&
\end{tabular}

Three students in this class who I think are unlikely to achieve well in maths in this class are

\begin{tabular}{|l|l|}
\hline Student's name & Main reason for your expectation \\
\hline & \\
\hline & \\
& \\
\hline & \\
& \\
\hline
\end{tabular}

Tick all options you consider to be true for you. I like to know about my students' families sports

family commitments

personalities

cultural activities

successes

other teachers (who they are)

progress in other subjects

other ( please specify)

Tick all the ethnic groups you are part of.

Māori

Pacific Nations (please indicate which)

NZ European

Asian (please indicate which)

other

List the languages you can speak

List the languages you speak at home 
Tick in the box to show how true each statement is for you.

I like to know about my students' cultural identities.

I like to know about the Māori world.

I like to know about Pacific Nations.

I like to use Māori examples in maths.

I like to use Pacific Nations examples in maths.

\begin{tabular}{|l|l|l|l|l|}
\hline $\begin{array}{l}\text { Strongly } \\
\text { Agree }\end{array}$ & Agree & Neutral & Disagree & $\begin{array}{l}\text { Strongly } \\
\text { Disagree }\end{array}$ \\
\hline & & & & \\
\hline & & & & \\
\hline & & & & \\
\hline & & & & \\
\hline & & & & \\
\hline & & & & \\
\hline
\end{tabular}

The main ways I establish rapport with this class are:

The main ways I show this class that I care about their maths progress are:

Three students in this class with whom I feel strong rapport are

\begin{tabular}{|l|l|}
\hline Student's name & Main reason for my feeling \\
\hline & \\
\hline & \\
\hline & \\
\hline & \\
\hline
\end{tabular}

Three students in this class with whom I feel weak rapport are

\begin{tabular}{|l|l|}
\hline Student's name & Main reason for my feeling \\
\hline & \\
\hline & \\
\hline & \\
& \\
\hline
\end{tabular}

The main challenges I face in establishing rapport with students in this class are: 
Do any of the school's rules or policies enhance the relationships you set up with students in this class?

Yes No

If yes, please explain

Do any of the school's rules or policies constrain or detract from the relationships you set up with students in this class?

Yes No

If yes, please explain

Do you incorporate aspects relevant to the students' school lives and experiences in your teaching?

Yes No

If so, in what ways?

Do you incorporate aspects relevant to the out of school lives and experiences of students in your teaching?
Yes
No

If so, in what ways? 
Tick the box to show how strongly each aspect informs your teaching of this class. What informs your teaching of this class?

Experiences teaching this class

Experiences teaching similar classes

Discussions with students from this class

Discussions with colleagues

Professional reading

Professional development

(Please explain)

Other

(Please explain)

\begin{tabular}{|l|l|l|l|l|}
\hline $\begin{array}{l}\text { Strongly } \\
\text { Agree }\end{array}$ & Agree & Neutral & Disagree & $\begin{array}{l}\text { Strongly } \\
\text { Disagree }\end{array}$ \\
\hline & & & & \\
\hline & & & & \\
\hline & & & & \\
\hline & & & & \\
\hline & & & & \\
\hline & & & & \\
\hline & & & & \\
\hline
\end{tabular}

Do you have any goals for the maths learning of this class this year?

Yes No

If so, what are your goals for their maths learning this year?

What is the main reason that these are your goals?

On average how much time do you spend preparing for a lesson with this class?

How does this time compare with the time you spend preparing for other classes?

Please tick how many years' teaching experience you have:

$0-10 \quad 11-20 \quad 21-30 \quad$ over 30

Thank you very much for your help in answering this questionnaire. 


\section{Appendix 8: Second teacher questionnaire}

Name:

How true are each of the following statements for you?

Tick the box that most closely shows how you feel.

I like teaching Year 10 Maths

I expect students in this class to work hard in Maths

I encourage students in this class to do their best

I like the class to ask questions

I care about the maths progress of this class

\begin{tabular}{|l|l|l|l|l|}
\hline $\begin{array}{l}\text { Strongly } \\
\text { Agree }\end{array}$ & Agree & Neutral & Disagree & $\begin{array}{l}\text { Strongly } \\
\text { Disagree }\end{array}$ \\
\hline & & & & \\
\hline & & & & \\
\hline & & & & \\
\hline & & & & \\
\hline & & & & \\
\hline
\end{tabular}

Tick all options that apply to you.

When students in this class work hard in Maths I believe it is because

their parents expect them to

they want to learn the work

they want to work well for me

their friends work hard

they know it will be useful for their jobs in the future

they know it will be useful for them in life other than for a job

they want to be successful in NCEA next year

other (please explain)

The most important ways I maintain rapport with this class are:

The main challenges I face in maintaining rapport with students in this class are: 
Does external assessment, such as NCEA, have any affect on your relationship with this class? Please comment.

Three students in this class who are achieving well in maths are

\begin{tabular}{|l|l|}
\hline Student's name & Main reason for their progress \\
\hline & \\
\hline & \\
\hline & \\
\hline & \\
\hline
\end{tabular}

Three students in this class who are achieving least well in maths are

\begin{tabular}{|l|l|}
\hline Student's name & Main reason you believe this is so \\
\hline & \\
\hline & \\
\hline & \\
\hline & \\
\hline
\end{tabular}

The most important ways I show this class that I care about their maths progress are: 
Show the proportion of the study class each statement is true for.

\begin{tabular}{|l|l|l|l|l|}
\hline \multicolumn{1}{|c|}{ I know something about my students' } & $\begin{array}{c}\text { All } \\
\text { students }\end{array}$ & $\begin{array}{c}\text { Most } \\
\text { students }\end{array}$ & $\begin{array}{c}\text { Some } \\
\text { students }\end{array}$ & $\begin{array}{c}\text { No } \\
\text { students }\end{array}$ \\
\hline families & & & & \\
\hline sports & & & & \\
\hline family commitments & & & & \\
\hline maths abilities & & & & \\
\hline personalities & & & & \\
\hline cultural identities & & & & \\
\hline cultural activities & & & & \\
\hline other teachers (who they are) & & & & \\
\hline successes & & & & \\
\hline progress in other subjects & & & & \\
\hline other & & & & \\
\hline $\begin{array}{l}\text { Students in this class want to improve their } \\
\text { maths skills this year }\end{array}$ & & & & \\
\hline $\begin{array}{l}\text { Students in this class are confident they can } \\
\text { do the work if they try. }\end{array}$ & & & & \\
\hline $\begin{array}{l}\text { Students in this class can succeed with Year } \\
\text { maths. }\end{array}$ & & & & \\
\hline maths in class want to learn Year 10 & & & & \\
\hline
\end{tabular}

Do you think it is important that you care about the students' maths learning? Give reasons for your answer. 
Three students in this class with whom I feel strong rapport are

\begin{tabular}{|l|l|}
\hline Student's name & Main reason for my feeling \\
\hline & \\
\hline & \\
\hline & \\
\hline & \\
\hline
\end{tabular}

Three students in this class with whom I feel least rapport are

\begin{tabular}{|l|l|}
\hline Student's name & Main reason for my feeling \\
\hline & \\
\hline & \\
\hline & \\
\hline & \\
\hline
\end{tabular}

How do you like to give feedback to students about their maths progress? 
Tick the box that most closely shows how you feel each aspect is for building and maintaining effective teacher-student relationships.

Smiling

Using humour

Rewarding good work

Knowing students' names

Having fun learning maths

Setting harder maths work

Showing I like teaching the class

Giving students choices

Being patient

Listening to students

Using praise

Using correct Māori and Pacific Nations pronunciation

\begin{tabular}{|l|l|l|l|l|}
\hline $\begin{array}{l}\text { Strongly } \\
\text { Agree }\end{array}$ & Agree & Neutral & Disagree & $\begin{array}{l}\text { Strongly } \\
\text { Disagree }\end{array}$ \\
\hline & & & & \\
\hline & & & & \\
\hline & & & & \\
\hline & & & & \\
\hline & & & & \\
\hline & & & & \\
\hline & & & & \\
\hline & & & & \\
\hline & & & & \\
\hline & & & & \\
\hline & & & & \\
\hline & & & & \\
\hline
\end{tabular}

Tick in the box to show how true each statement is for you.

I know about the Māori world.

I know about Pacific Nations.

I use Māori examples in maths.

I use Pacific Nations examples in maths.

\begin{tabular}{|l|l|l|l|l|}
\hline $\begin{array}{l}\text { Strongly } \\
\text { Agree }\end{array}$ & Agree & Neutral & Disagree & $\begin{array}{l}\text { Strongly } \\
\text { Disagree }\end{array}$ \\
\hline & & & & \\
\hline & & & & \\
\hline & & & & \\
\hline & & & & \\
\hline
\end{tabular}

Thank you very much for completing this questionnaire. 


\section{Appendix 9: Third teacher questionnaire}

Name:

How true are each of the following statements for you?

Tick the box that most closely shows how you feel.

I like teaching Year 10 Maths

I expect students in this class to work hard in Maths

I encourage students in this class to do their best

I like the class to ask questions

I care about the maths progress of this class

Most students in this class prefer to get feedback on their maths achievement privately.

\begin{tabular}{|l|l|l|l|l|}
\hline $\begin{array}{c}\text { Strongly } \\
\text { Agree }\end{array}$ & Agree & Neutral & Disagree & $\begin{array}{c}\text { Strongly } \\
\text { Disagree }\end{array}$ \\
\hline & & & & \\
\hline & & & & \\
\hline & & & & \\
\hline & & & & \\
\hline & & & & \\
\hline & & & & \\
\hline
\end{tabular}

Tick all options that apply to you.

When students in this class work hard in Maths I believe it is because

their parents expect them to

they want to learn the work

they want to work well for me

their friends work hard

they know it will be useful for their jobs in the future

they know it will be useful for them in life other than for a job

they want to be successful in NCEA next year

other (please explain)

Three students in this class who are achieving well in maths are

\begin{tabular}{|l|l|}
\hline Student's name & Main reason for their progress \\
\hline & \\
\hline & \\
\hline & \\
\hline
\end{tabular}


Three students in this class who are achieving least well in maths are

\begin{tabular}{|l|l|}
\hline Student's name & Main reason you believe this is so \\
\hline & \\
\hline & \\
\hline & \\
\hline
\end{tabular}

Show the proportion of the study class each statement is true for.

I know something about my students'

families

sports

family commitments

maths abilities

personalities

cultural identities

cultural activities

other teachers (who they are)

successes

progress in other subjects

other

\begin{tabular}{|l|c|c|c|}
\hline $\begin{array}{c}\text { All } \\
\text { students }\end{array}$ & $\begin{array}{c}\text { Most } \\
\text { students }\end{array}$ & $\begin{array}{c}\text { Some } \\
\text { students }\end{array}$ & $\begin{array}{c}\text { No } \\
\text { students }\end{array}$ \\
\hline & & & \\
\hline & & & \\
\hline & & & \\
\hline & & & \\
\hline & & & \\
\hline & & & \\
\hline & & & \\
\hline & & & \\
\hline & & & \\
\hline & & & \\
\hline
\end{tabular}

Students in this class want to improve their maths skills this year

Students in this class are confident they can do the work if they try.

Students in this class can succeed with Year 10 maths.

Students in this class want to learn Year 10 maths.

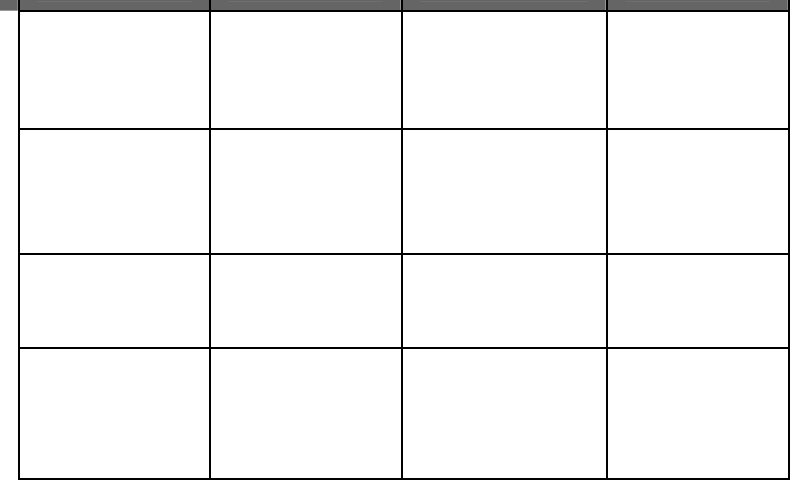

Do you find it difficult to include Māori and Pacific Nations contexts in your maths teaching?

Yes No Not applicable, I do not aim to do this 
If yes, what are the difficulties you experience in doing this?

Three students in this class with whom I feel strongest rapport are

\begin{tabular}{|l|l|}
\hline Student's name & Main reason for my feeling \\
\hline & \\
\hline & \\
\hline & \\
\hline & \\
\hline
\end{tabular}

Three students in this class with whom I feel least rapport are

\begin{tabular}{|l|l|}
\hline Student's name & Main reason for my feeling \\
\hline & \\
\hline & \\
\hline & \\
\hline & \\
\hline
\end{tabular}

If you can, please list things special to your own culture. 
Do you have a position of pastoral care for this year level e.g., dean?

Yes No

If yes,

1) does having this role have any effect on the relationships you have with students in this class?

Yes, a positive effect overall

Yes, a negative effect overall

No, no effect

Please explain

2) does having this role have any effect on the maths achievement of this class?

Yes, a positive effect overall

Yes, a negative effect overall

No, no effect

Please explain

If no,

1) do you believe having a role of pastoral care for the year level would have had any effect on the relationships you have with students in this class?

Yes, a positive effect overall Yes, a negative effect overall No, no effect

Please explain

2) do you believe having such a role would have any effect on the maths achievement of this class?

Yes, a positive effect overall Yes, a negative effect overall No, no effect

Please explain 
Tick the box that most closely shows how you feel.

I give written feedback to students about their maths progress.

I tell students about their maths progress.

Students in this class ask me about their maths progress.

\begin{tabular}{|l|l|l|l|}
\hline Often & Sometimes & $\begin{array}{c}\text { Not } \\
\text { often }\end{array}$ & Never \\
\hline & & & \\
\hline & & & \\
\hline & & & \\
& & & \\
\hline
\end{tabular}

Some schools demonstrate clearly that students' ethnicities/cultures are important to them and others don't.

Place a tick on the line to show how your school treats students' ethnicities/cultures.

I don't know if students' cultures are important to my school.

\section{Students'}

cultures are not important at all at our school

If you can, please give an example of how your school shows this.

Some teachers demonstrate clearly that students' ethnicities/cultures are important to them and others don't.

Place a tick on the line to show how you think about students' ethnicities/cultures.

1

Students'

cultures are

Students'

very important to

cultures are

me

not important

at all to me

If you can, please give an example of how you show this. 
It is important to this class that I care about their ethnicities/cultures.

My ethnicity/culture is not relevant when I am at school.

My ethnicity/culture is not relevant when I am teaching maths.

\begin{tabular}{|l|l|l|l|l|}
\hline $\begin{array}{c}\text { Strongly } \\
\text { Agree }\end{array}$ & Agree & Neutral & Disagree & $\begin{array}{c}\text { Strongly } \\
\text { Disagree }\end{array}$ \\
\hline & & & & \\
\hline & & & & \\
\hline & & & & \\
\hline
\end{tabular}

Did you notice any surprising results in the student data from Term 1 and 2?

Yes

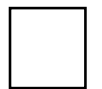

No

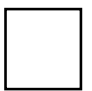

Don't know

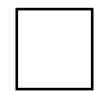

If so, what were they? Why did they surprise you?

Did you notice any differences in your maths lessons after the data from Term 1 and 2 were shared?

Yes

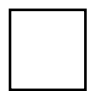

No

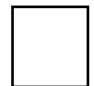

Don't know

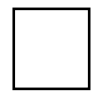

If so, what were they? What were the reasons for this?

Do you think it is important for maths learning that you care about students' cultures?

Yes

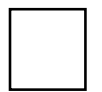

No

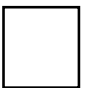

Don't know

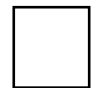

Please give reasons for your answer 
Every person is a part of lots of different groups. Below are some groups you might belong to.

On the first diagram please show how important each of these groups is to you in your life.

Use $\quad \sqrt{ }$ to show this group is a bit important to you,

$\sqrt{ }$ to show this group is quite important to you,

$\sqrt{ } \sqrt{ }$ to show this group is very important to you,

D to show you do not belong to a group like this.

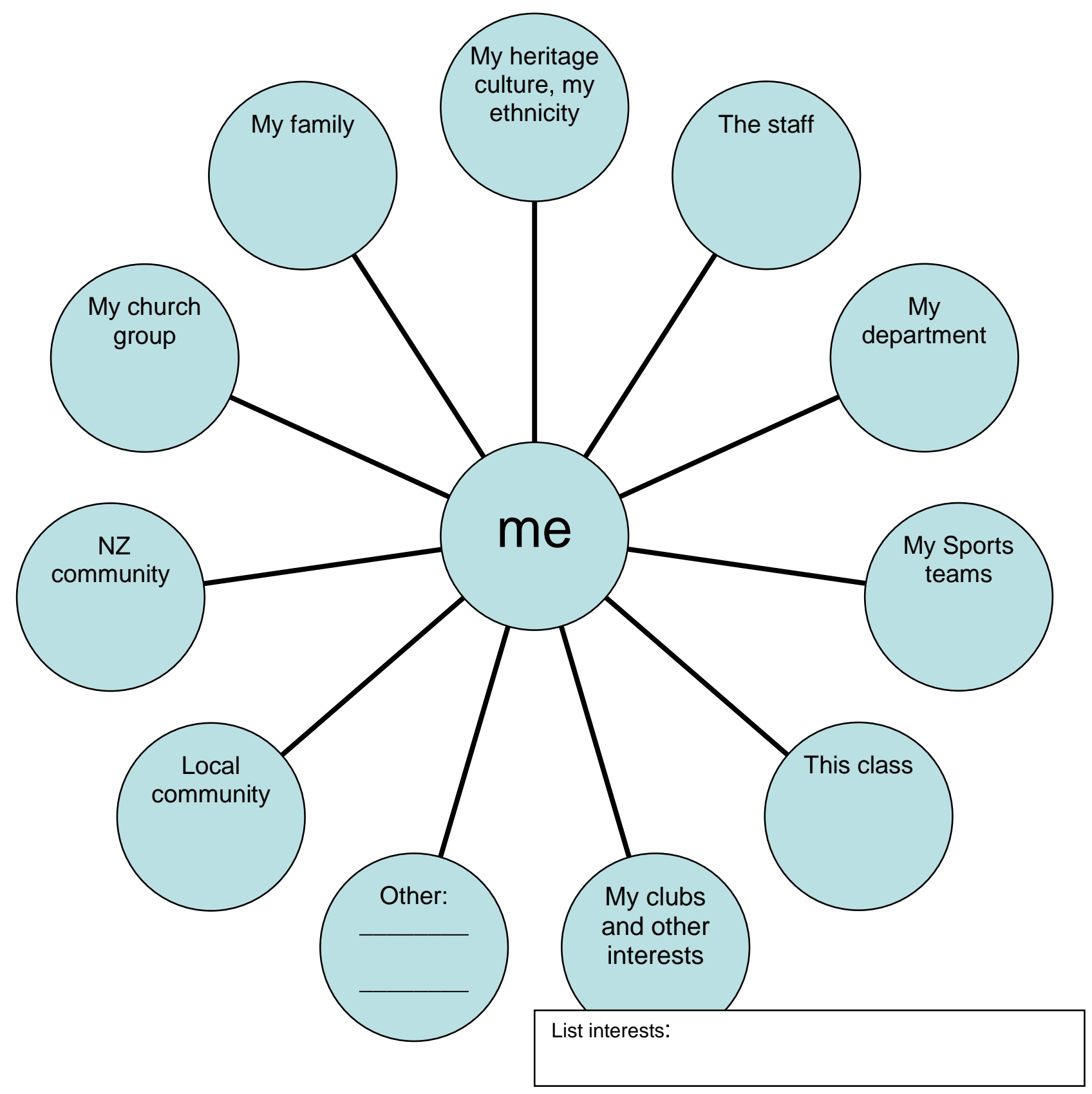


On the second diagram show how important each of these groups is to you when you are teaching maths.

Use $\sqrt{ }$ to show this group is a bit important to you when you are teaching maths,

$\sqrt{ }$ to show it is quite important to you when you are teaching maths,

$\sqrt{ } \sqrt{ }$ to show the group is very important to you when you are teaching maths,

$\mathrm{X}$ to show the group is not relevant when you are teaching maths.

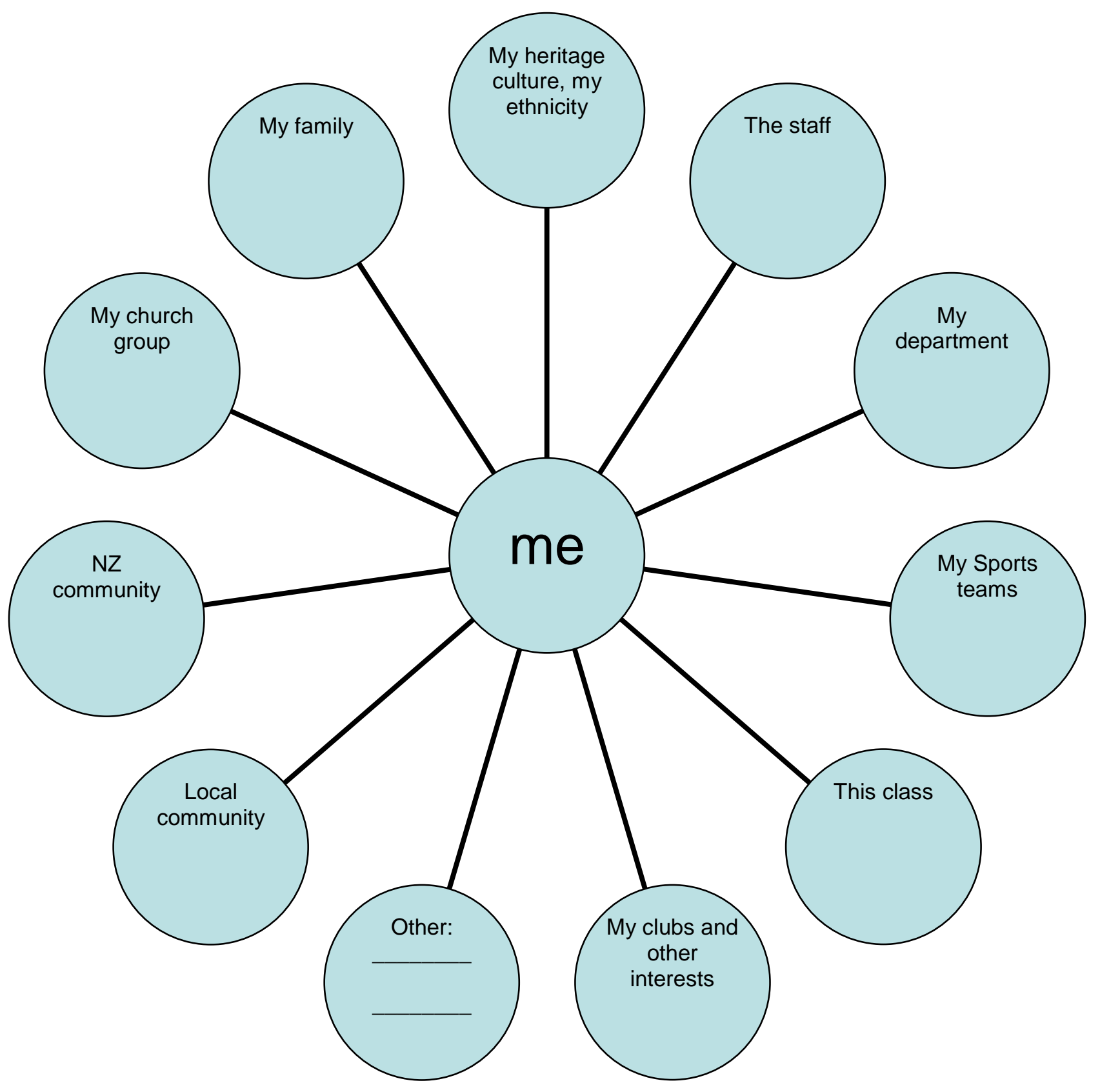

Thank you very much for your help in this project. 


\section{Appendix 10: Student interviews}

Note: Interviews were semistructured. Probing questions were used to explore responses.

Student Interview 1

Describe the maths lesson you have enjoyed most so far this year and try to explain why you enjoyed it.

What do you think students need to learn in maths?

What do you think students need to be successful at maths?

What do you think makes a good maths teacher?

Do you think maths achievement is important for this class? Why? Why not?

How important do you think is it for your teacher to know about your maths progress?

Is it important to you for your teacher to show they care about your maths progress? What makes you think so?

Is it important to you for your teacher to know about you as a person?

Is it important to you for your teacher to show you that they care about you as a person?

How important to you is it for your teacher to know about your cultural identity?

Is it important to you for your teacher to show you that they care about your cultural identity?

How important is a good relationship with your teacher for your mathematics learning?

Can you tell me what you know about your teacher? Is there anything else you would like to know? How could you find out this information? 


\section{Student Interview 2}

Describe the type of maths lesson you've enjoyed most this year and why you enjoyed it.

Has your attitude to learning maths changed since the start of the year?

Has your success in maths changed since the start of this year?

How does your teacher encourage you?

How would you like your teacher to encourage you?

Does your teacher care about you as a person? How does this compare to other subjects?

Does your teacher care about your maths progress? How does this compare to other subjects?

Does your teacher believe in your ability to be successful at maths?

What are your teacher's main ways for getting on with this class?

Do you think your class gets on well with the teacher? If so, what are your class' main ways of getting on with the teacher?

What do you see as the main challenges for your teacher in getting on with this class?

How is learning maths different from learning other subjects?

Do you think this class is the right level for you? 


\section{Student Interview 3}

Do you like learning more when you are working on your own or with others? Why is this?

Are there any things that stop you learning well in maths? If so, what are they?

Does your teacher use maths examples that relate to your everyday life? How do you feel about this?

Has your attitude to learning maths changed since the start of the year? Why do you think this is so?

Has your success in maths changed since the start of this year?

On the second circle diagram in the questionnaire you indicated that some groups were more relevant to your maths learning than others. Can you choose one group you thought was important for your maths learning and explain why you think it is important.

Can you choose one group you thought was not important for your maths learning and explain why you think it is not important.

a) For SS and WW's students: Your maths teacher this year is also your dean. Do you think this was helpful for your maths learning, not helpful for your maths learning or didn't make any difference either way?

b) For UU, WK, ST, and UM's students: Your maths teacher this year is not your dean. Do you think it would be helpful for your maths learning if your maths teacher was also your dean, not helpful for your maths learning or wouldn't make any difference either way?

How do you feel about having been involved in this project this year? Do you have any suggestions for this project as it continues into next year? 


\section{Appendix 11: Teacher interviews}

Note: Interviews were semistructured. Probing questions were used to explore responses.

Teacher Interview 1

How important do you believe good teacher-student relationships are for mathematics learning?

How do you plan to get to know the students?

Are you thinking about or planning how you will establish your relationships with students in the class? If yes, what are your plans?

How important do you feel it is to know about your students' maths progress?

Do you feel it is important to show students that you care about their maths progress? What makes you think so?

How important do you feel it is for you to know about your students as individuals?

Do you feel it is important to show students that you care about them as individuals?

How important do you feel it is to know about your students' cultural identities?

Do you feel it is important to show the students that you care about their cultural identities?

Do you think maths achievement is important for this class?

Was it a school policy to keep this class with the same teacher for 2 years? Please explain. (Teachers WW and WK only) 
Describe the maths lesson you have enjoyed most with this class so far and why you enjoyed it.

What are your main strategies for developing rapport with this class?

What do you see as the main challenges to establishing rapport with this class?

In the questionnaire you indicated your confidence in your knowledge of the maths progress of students in this class?

In the questionnaire you indicated aspects you would like to know about your students. How would you like to find out this information?

In the questionnaire you indicated how you feel about knowing the students' cultural identities. How would you like to find out this information?

Do you relate classroom maths learning for this class to ideas from Māori and/or Pacific Nation cultures? How do you feel about doing this?

Do you relate classroom maths learning for this class to students' everyday lives (in and out of school)? How do you feel about doing this?

Please tell me what you know about (student with good rapport as recorded on teacher questionnaire).

Please tell me what you know about on teacher questionnaire). (student with little rapport as recorded

Is there a school wide ethic of care at your school? Can you give some examples to support your view?

Can you remember a teacher who taught you who cared about you as a person, or about your maths learning, or about your cultural identity? How did you know that they cared?

Do you think your involvement with this project has affected how you teach this class? If so, how? 
Teacher Interview 3

Describe the type of maths lesson you enjoy the most with this class and why you enjoy it the most.

What personal qualities do students need to have to be successful at maths?

What makes a good maths teacher?

Has your attitude to teaching this class changed since the start of the year? If so, how?

How do you encourage students in this class?

What do you think students need to learn in maths?

Has your relationship with any individual student/s changed markedly since the beginning of the year? If so, please explain how it changed, why you think it changed in this way and any associated effects on attitude and/or achievement you have noticed.

How is teaching maths different from teaching other subjects?

How is teaching maths different from teaching other subjects with respect to developing and maintaining relationships with students?

What are the barriers for your students who are working very well in maths to achievement in maths internal and external assessment? 
What are your school's expectations in terms of teachers showing evidence towards meeting the Professional Standards?

How difficult is it to address the professional standard regarding the Treaty of Waitangi in your teaching of this class? What are the difficulties?

Last term I shared the summary of students' questionnaire results from Terms 1 and 2. Does anything from the results come to mind now? Are you aware of having altered your teaching as a result of seeing this data? If so, how?

Last term I sent you a draft paper which I hope to have published on some of the initial study results. Does anything from the paper come to mind now? Are you aware of having altered your teaching as a result of seeing this paper? If so, how?

In the questionnaire in Term1 you indicated your goals for teaching this class this year. (I will share with them the goals they set.) Did your goals change over the year? To what extent have you succeeded with these goals?

How do you feel about having been involved in this project this year? Do you have any suggestions for this project as it continues into next year? 


\section{Appendix 12: Crosschecking themes}

Themes were explored across the interviews, questionnaires, and observation schedule in order to ensure triangulation would be achieved and that the tools would enable the research questions to be answered. Two gaps were identified and questions regarding barriers to learning and teachers' use of real life contexts added to the third student questionnaire.

Note: Due to subsequent changes to the data gathering tools (e.g., addition of further questions) made as the study progressed, in light of the data gathered and further cultural advice, slight discrepancies between the question references in the tables and those in the data gathering tools exist.

\section{Interview Questions}

Key: Questions are referred to in the format x.y as follows: $x$ represents $1^{\text {st }}, 2^{\text {nd }}$, or 3rd interview, and $y$ represents the question number of the interview.

\begin{tabular}{|c|c|c|}
\hline Theme & Student Questions & Teacher Questions \\
\hline Lesson most enjoyed & $1.1,2.1,3.1$ & 2.1 \\
\hline \multicolumn{3}{|l|}{ Teacher Qualities } \\
\hline What makes a good teacher & 1.4 & 3.2 \\
\hline Knowing about progress & 1.6 & $1.4,2.4$ \\
\hline Caring about progress & $1.7,2.6,3.5$ & 1.5 \\
\hline Knowing about person & 1.8 & $1.2,1.6,2.5$ \\
\hline Caring about person & $1.9,2.5,3.4$ & 1.7 \\
\hline Knowing about cultural identity & 1.10 & $1.8,2.6$ \\
\hline Caring about cultural identity & 1.11 & 1.9 \\
\hline Belief in ability to do maths & $2.8,3.7$ & \\
\hline \multicolumn{3}{|l|}{ Learning Maths } \\
\hline $\begin{array}{l}\text { Importance of good relationship with } \\
\text { teacher for good learning }\end{array}$ & 1.12 & $1.1,1.3$ \\
\hline What maths you need to learn & 1.2 & 3.4 \\
\hline $\begin{array}{l}\text { Qualities needed to be successful in } \\
\text { maths }\end{array}$ & 1.3 & 3.1 \\
\hline $\begin{array}{l}\text { Importance of maths achievement for } \\
\text { the class }\end{array}$ & 1.5 & 1.10 \\
\hline Use of Māori and/or Pacific contexts & $2.7,3.6$ & 2.7 \\
\hline Relating to everyday life & 3.3 & 2.8 \\
\hline Differences to other subjects & 2.4 & $3.6,3.7$ \\
\hline Barriers to achievement & 3.2 & 3.5 \\
\hline \multicolumn{3}{|l|}{ Encouragement } \\
\hline Teacher encouragement & $2.2,2.3$ & 3.3 \\
\hline \multicolumn{3}{|l|}{ Teacher-class relationship } \\
\hline Ways to get on with class & 2.9 & 2.2 \\
\hline Challenges to rapport & 2.11 & 2.3 \\
\hline Ways to get on with teacher & 2.10 & \\
\hline
\end{tabular}




\section{Questionnaires}

Key: Questions are referred to in the table using a digit (represents the page number of the question) and a letter (representing the question on the page).

\begin{tabular}{|c|c|c|}
\hline Theme & Student Questions & Teacher Questions \\
\hline \multicolumn{3}{|l|}{ Lesson most enjoyed } \\
\hline \multicolumn{3}{|l|}{ Teacher Qualities } \\
\hline What makes a good teacher & $1 \mathrm{f}, 1 \mathrm{~g}$ & \\
\hline Knowing about progress & $1 \mathrm{k}$ & $1 \mathrm{~h}$ \\
\hline Caring about progress & $1 \mathrm{c}$ & $3 b$ \\
\hline Knowing about person & $2 a$ & $2 b$ \\
\hline Caring about person & $1 \mathrm{~d}, 1 \mathrm{~h}, 1 \mathrm{i}$ & \\
\hline Knowing about cultural identity & $3 a$ & $2 \mathrm{e}$ \\
\hline \multicolumn{3}{|l|}{ Caring about cultural identity } \\
\hline Belief in ability to do maths & & $1 \mathrm{i}$ \\
\hline Know about Māori & $3 b$ & $2 f$ \\
\hline Know about PN & $3 c$ & $2 g$ \\
\hline \multicolumn{3}{|l|}{ Learning Maths } \\
\hline $\begin{array}{l}\text { Importance of good relationship with } \\
\text { teacher for good learning }\end{array}$ & & $1 d$ \\
\hline \multicolumn{3}{|l|}{ What maths you need to learn } \\
\hline $\begin{array}{l}\text { Qualities needed to be successful in } \\
\text { maths }\end{array}$ & & $1 \mathrm{k}, 2 \mathrm{a}$ \\
\hline $\begin{array}{l}\text { Importance of maths achievement for the } \\
\text { class }\end{array}$ & & $1 \mathrm{~g}$ \\
\hline Use of Māori and/or Pacific contexts & $3 d$ & $2 \mathrm{~h}, 2 \mathrm{i}$ \\
\hline Relating to everyday life & & $1 c, 4 d, 5 a$ \\
\hline \multicolumn{3}{|l|}{ Differences to other subjects } \\
\hline \multicolumn{3}{|l|}{ Barriers to achievement } \\
\hline \multicolumn{3}{|l|}{ Encouragement } \\
\hline Teacher encouragement & $1 \mathrm{e}$ & \\
\hline \multicolumn{3}{|l|}{ Teacher-class relationship } \\
\hline Ways to get on with class & & $\begin{array}{l}\text { 3a, } 4 \mathrm{~b}, \\
\text { NCEA ( } 2^{\text {nd }} \text { and } 3^{\text {rd }} \\
\text { questionnaire only) }\end{array}$ \\
\hline Challenges to rapport & & $\begin{array}{l}\text { 4a, 4c, } \\
\text { NCEA ( } 2^{\text {nd }} \text { and } 3^{\text {rd }} \\
\text { questionnaire only) }\end{array}$ \\
\hline Ways to get on with teacher & $3 e$ & \\
\hline Teacher-student rapport & & $3 c, 3 d$ \\
\hline
\end{tabular}




\begin{tabular}{|c|c|c|}
\hline \multicolumn{3}{|l|}{ Personal Information } \\
\hline Male/female & 1 & \\
\hline Ethnicity & $2 b$ & 2c \\
\hline Languages & $2 \mathrm{c}, 2 \mathrm{~d}$ & $2 d$ \\
\hline Culture important & $1 \mathrm{j}$ & \\
\hline Personal maths learning beliefs & $1 \mathrm{a}, 1 \mathrm{~b}, 1 \mathrm{l}, 1 \mathrm{~m}, 1 \mathrm{n}$ & $\begin{array}{l}1 a, b, e, f, j, 5 b, 5 c \\
6 a, 6 b\end{array}$ \\
\hline Teaching experience & & $6 d$ \\
\hline Age group & & $6 c$ \\
\hline
\end{tabular}




\section{Observations}

Key:

$\checkmark$ indicates presence in observation schedule

\begin{tabular}{|l|c|}
\hline \multicolumn{1}{|c|}{ Theme } & Present in schedule \\
\hline Lesson most enjoyed & \\
\hline Teacher Qualities & \\
\hline What makes a good teacher & $\checkmark$ \\
\hline Knowing about progress & $\sqrt{ }$ \\
\hline Caring about progress & $\sqrt{ }$ \\
\hline Knowing about person & $\sqrt{ }$ \\
\hline Caring about person & $\sqrt{ }$ \\
\hline Knowing about cultural identity & $\sqrt{ }$ \\
\hline Caring about cultural identity & $\sqrt{ }$ \\
\hline Belief in ability to do maths & $\sqrt{ }$ \\
\hline Know about Māori & \\
\hline Know about PN & \\
\hline \hline Learning Maths & \\
\hline $\begin{array}{l}\text { Importance of good relationship with teacher } \\
\text { for good learning }\end{array}$ & \\
\hline What maths you need to learn & $\checkmark$ \\
\hline Qualities needed to be successful in maths & \\
\hline Importance of maths achievement for the class & \\
\hline Use of Māori and/or Pacific contexts & $\sqrt{ }$ \\
\hline Relating to everyday life & \\
\hline Differences to other subjects & \\
\hline Barriers to achievement & \\
\hline \hline Encouragement & \\
\hline Teacher encouragement & \\
\hline \hline Teacher-class relationship & \\
\hline Ways to get on with class & \\
\hline Challenges to rapport & \\
\hline Ways to get on with teacher & \\
\hline Teacher-student rapport & \\
\hline \hline
\end{tabular}




\section{Appendix 13: Participant information and consent form (sample)}

Note: Similarly worded information letters and consent forms were used for teachers, parents/guardians, and students.

Robin Averill

Mathematics Education Department

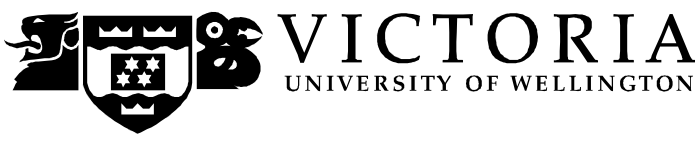

Victoria University of Wellington College of Education

PO Box 17-310

Karori

Wellington

February 2007

Phone: 4639714

\section{Information Sheet for Study on Teacher-student Relationships (Principal's copy)}

I am a Senior Lecturer in Mathematics Education at Victoria University of Wellington College of Education. I am doing research into teacher-student relationships in junior secondary school Mathematics. This research will form the basis of my PhD thesis at Victoria University of Wellington. Megan Clark is the supervisor for this research.

I very much hope that you will agree to your school participating in this research. The university requires that ethics approval be obtained for research involving people and that people involved with the research are given written information about it and give their written consent to be involved.

\section{Purpose of the Research:}

This PhD project explores:

- positive classroom teacher-student relationships in Year 9 and 10 mathematics classrooms; and

- the connection between such relationships and mathematics learning.

\section{Process of the Research:}

The research involves data gathering through short questionnaires, short interviews and audio tapes and observing classroom lessons for the first 2 to 3 weeks of Term 1 , and a week in the middle of each of Terms 2 and 3 of 2007.

All data will be kept confidential. No person other than my supervisor and myself will see this data. The identity of the school and all participants (students, teacher, head of department, principal) will be concealed and will be known only to myself and my supervisor. All data will be destroyed within two years of completion of the project.

Anyone has the right to withdraw from this project at any time. The Human Ethics Committee of Victoria University has approved this research proposal. The completed thesis will be submitted for assessment to The School of Mathematics, Statistics and Computer Science and put in the University Library.

Feedback on the research findings will be provided to interested participating schools. The research results will be published in my thesis, in conference papers, and journal articles and will inform teacher education programmes. 
If you would like further information about this study please contact me (ph 463 9714), or my supervisor Megan Clark, (School of Mathematics, Statistics and Computer Science at Victoria University, PO Box 600, Wellington).

If you are happy for your school to be part of this study please sign and return the attached consent form.

Yours sincerely

Robin Averill

Senior Lecturer: Mathematics Education

Victoria University of Wellington College of Education

\section{VICTORIA UNIVERSITY OF WELLINGTON COLLEGE OF EDUCATION CONSENT TO PARTICIPATE IN RESEARCH (PRINCIPAL)}

\section{Positive Student Teacher Relationships in Year 9 and 10 Mathematics}

I have been given and have understood the information about this research project. I have had an opportunity to ask questions and have them answered to my satisfaction. I understand that I may withdraw my school from this study (before data collection and analysis is complete) without having to give a reason of any sort.

I understand that any information provided will be kept confidential to the researcher (Robin Averill) and the project supervisor (Megan Clark), that the published results will not use my name or my school's name, and that none of the information collected will be presented in any way that will identify any individuals. I understand that at the end of the project all data gathered will be destroyed.

I understand that the data provided will not be used for any purpose other than those explained in the research information sheet.

I would like to receive a summary of the results of the research when it is finished.

I agree that my school is able to take part in this research.

Signed

Name

School

Date 


\section{Appendix 14: Typical lesson (UM)}

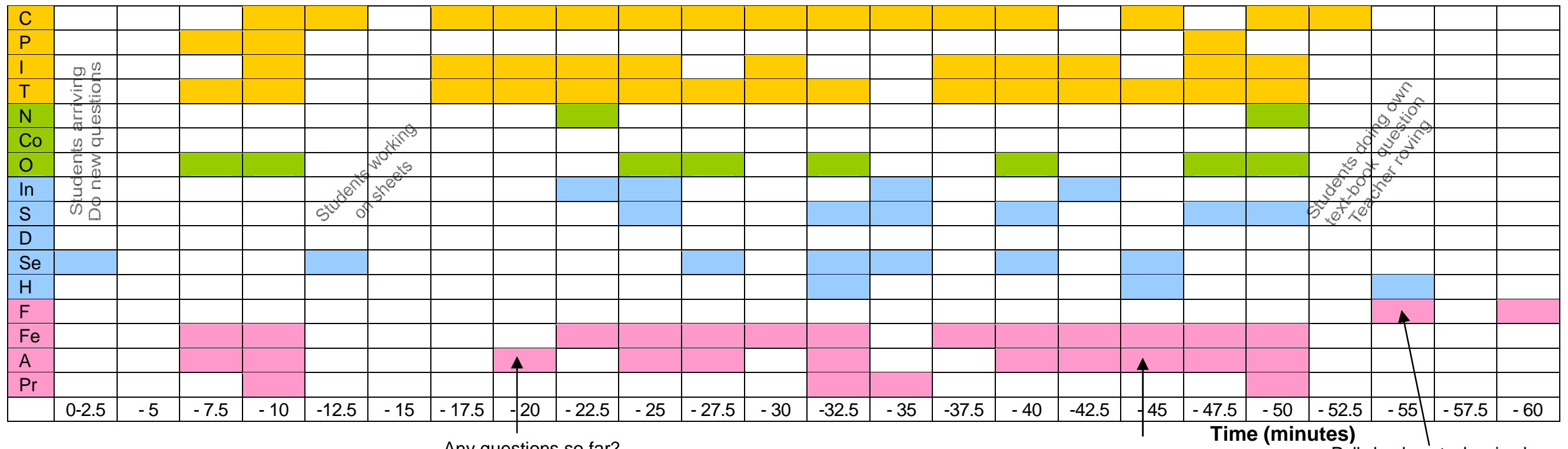

Any questions so far?

Notes: Shading has been used to highlight the four types of practice.

The analysis method used was adapted from an audiotape analysis process used by Thorpe (2007).

\begin{tabular}{|l|l|l|}
\hline \multirow{4}{*}{$\begin{array}{l}\text { Supporting new } \\
\text { mathematical learning }\end{array}$} & C & Co-construction, negotiation of learning \\
\cline { 2 - 3 } & P & Prior knowledge \\
\cline { 2 - 3 } & I & Instruction, teaching rather than telling \\
\cline { 2 - 3 } & T & Teacher questioning, questions from teacher to whole class or to individuals \\
\hline Reinforcing mathematical & N & Narrative, committed, connected, participatory teaching \\
\cline { 2 - 3 } & Co & Co-operative learning \\
\cline { 2 - 3 } & $\mathrm{O}$ & Oral language/literacy, oral rich atmosphere \\
\hline $\begin{array}{l}\text { Ensuring relevance of } \\
\text { mathematical learning }\end{array}$ & In & Integrated curricula, context match students' interests \\
\cline { 2 - 3 } & S & Student-generated questioning \\
\cline { 2 - 3 } & D & Differentiated learning \\
\cline { 2 - 3 } & Se & Self, involving personal identities \\
\cline { 2 - 3 } & H & Humour \\
\hline \multirow{5}{*}{$\begin{array}{l}\text { Giving encouragement, } \\
\text { assistance, and feedback }\end{array}$} & F & Feed-forward academic \\
\cline { 2 - 3 } & Fe & Feed-back academic \\
\cline { 2 - 3 } & $\mathrm{A}$ & Ako, reciprocity, teaching as partner in conversation of learning \\
\cline { 2 - 3 } & $\mathrm{Pr}$ & Praise \\
\hline
\end{tabular}

Does it make sense?

\section{Teacher UM}

Typical lesson (Term 2, lesson 1 - afternoon) Notes:

UM maintained the pace of the lesson (set time limits)

- Question review, question review style lesson, lots of maths learning discussion

- Students confident to answer, discuss maths, and ask questions

Shows care for maths learning through persistence 
Appendix 15: Exemplary lesson (SS)

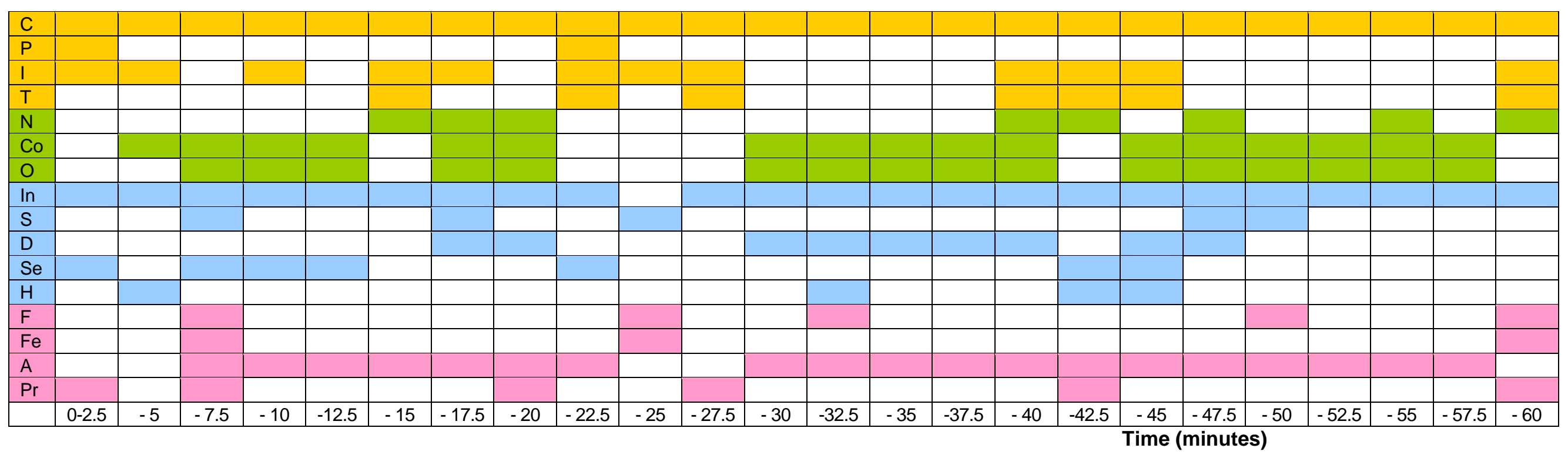

Notes: Shading has been used to highlight the four types of practice.

The analysis method used was adapted from an audiotape analysis process used by Thorpe (2007).

\begin{tabular}{|l|l|l|}
\hline \multirow{4}{*}{$\begin{array}{l}\text { Supporting new } \\
\text { mathematical learning }\end{array}$} & C & Co-construction, negotiation of learning \\
\cline { 2 - 3 } & P & Prior knowledge \\
\cline { 2 - 3 } & I & Instruction, teaching rather than telling \\
\cline { 2 - 3 } & T & Teacher questioning, questions from teacher to whole class or to individuals \\
\hline Reinforcing mathematical & N & Narrative, committed, connected, participatory teaching \\
\cline { 2 - 3 } & Co & Co-operative learning \\
\cline { 2 - 3 } & O & Oral language/literacy, oral rich atmosphere \\
\hline $\begin{array}{l}\text { Ensuring relevance of } \\
\text { mathematical learning }\end{array}$ & In & Integrated curricula, context match students' interests \\
\cline { 2 - 3 } & S & Student-generated questioning \\
\cline { 2 - 3 } & D & Differentiated learning \\
\cline { 2 - 3 } & Se & Self, involving personal identities \\
\cline { 2 - 3 } & H & Humour \\
\hline \multirow{5}{*}{$\begin{array}{l}\text { Giving encouragement, } \\
\text { assistance, and feedback }\end{array}$} & F & Feed-forward academic \\
\cline { 2 - 3 } & Fe & Feed-back academic \\
\cline { 2 - 3 } & $\mathrm{A}$ & Ako, reciprocity, teaching as partner in conversation of learning \\
\cline { 2 - 3 } & $\mathrm{Pr}$ & Praise \\
\hline
\end{tabular}

\section{Teacher SS}

Excellent lesson (Term 4, lesson 1 -

afternoon)

Notes:

- Rich mathematical tasks

- D by nature of the tasks

- Lots of laughter

- Lots of on-task behaviour 